
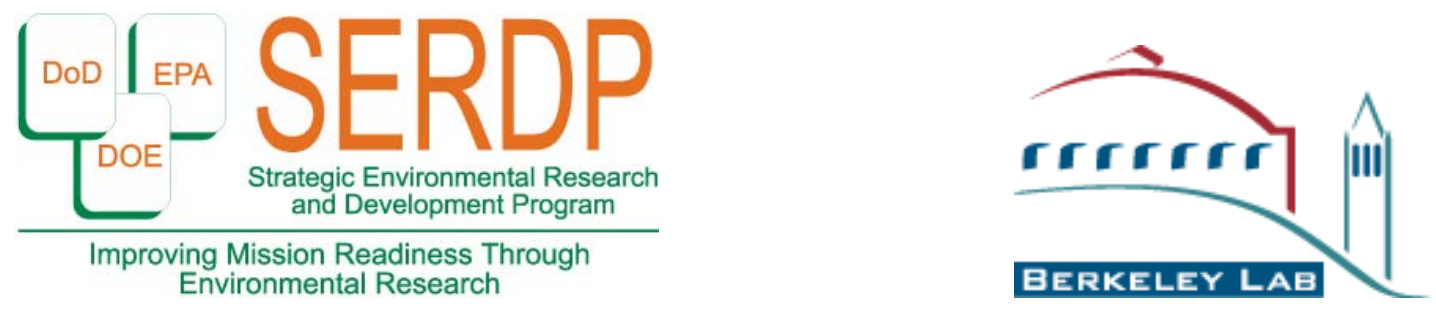

Final Report

\author{
DETECTION AND CLASSIFICATION \\ OF BURIED METALLIC OBJECTS \\ UX-1225
}

\author{
Frank Morrison, Torquil Smith, Alex Becker, \\ and Erika Gasperikova
}

Lawrence Berkeley National Laboratory

\title{
March 2005
}

p.o.c.: LBNL, 1 Cyclotron Road, MS:90-1116, Berkeley, CA 94720.

E-mail: EGasperikova@lbl.gov

This work was supported by the Office of Management, Budget and Evaluation, of the U.S. Department of Energy under Contract No. DE-AC03-76SF00098, and U.S. Department of Defense under Strategic Environmental Research and Development Program Project No. UX-1225 and Contract Nos. W74RDV093447299 and W74RDV30452524. 


\section{TABLE OF CONTENTS}

\section{Page}

1. INTRODUCTION ...........................................................................................................4

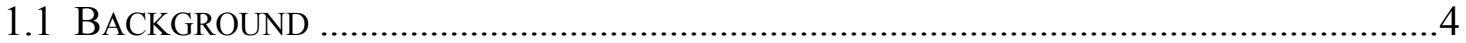

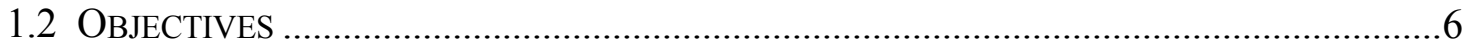

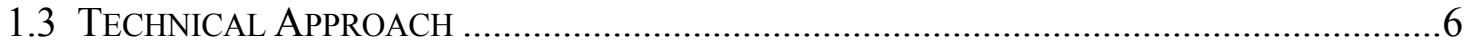

2. INVERSION FOR TARGET PARAMETERS AND THE ROLE OF SYSTEM

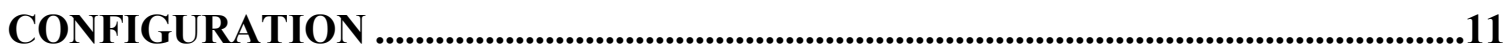

2.1 Estimating Equivalent Dipole Polarizabilities for the Inductive ResPonse

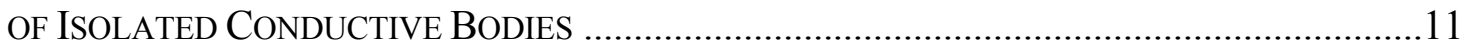

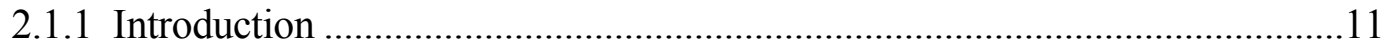

2.1.2 Estimating Dipole Polarizabilities When Object Center is Known.................14

2.1.3 Estimating Dipole Polarizabilities and Object Center Location .....................14

2.1.4 Estimating Dipole Polarizability Uncertainties .............................................15

2.1.5 Principal Moment and Principal Direction Uncertainties ...............................17

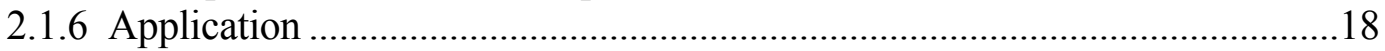

2.2 DEPTHS OF EQUIVALENT DIPOLE POLARIZABILITY RESOLUTION FOR SOME

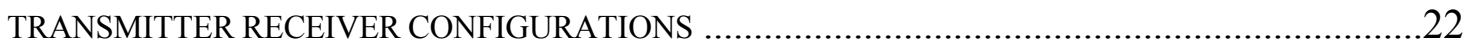

3. PERFORMANCE ASSESSMENT AND DIRECT OPTIMIZATION......................32

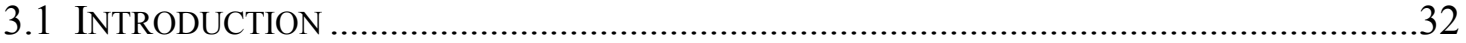

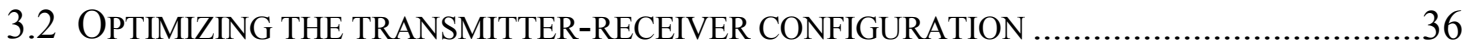

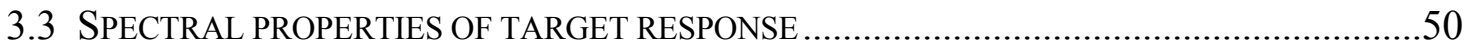

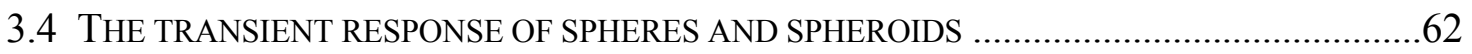

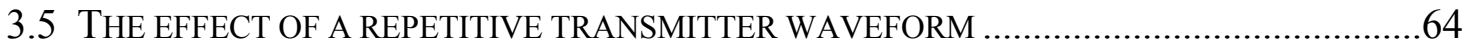

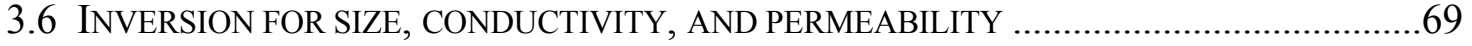

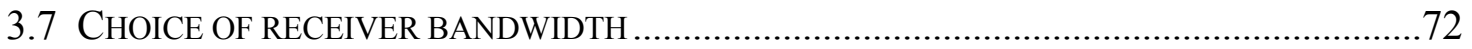

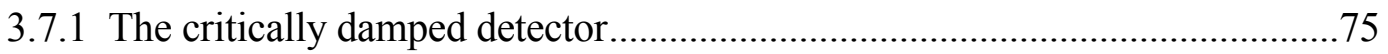

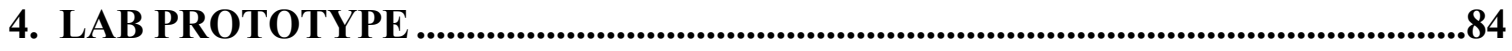

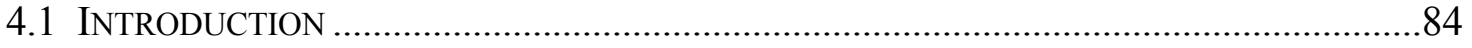

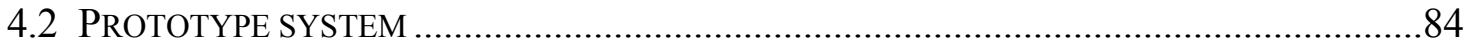

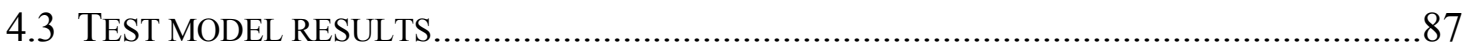

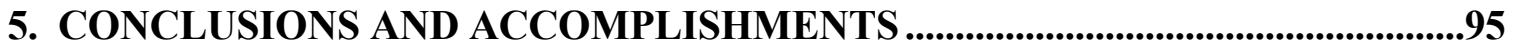

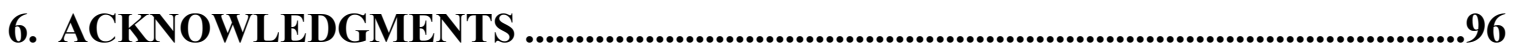

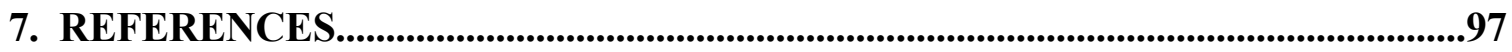

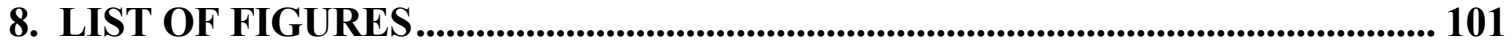

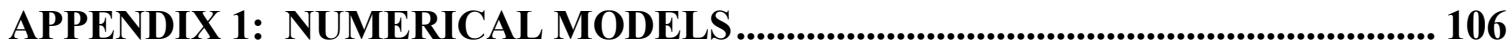

A.1.1 IMPROVEMENTS TO THE WEICHMAN-BLACKHAWK MFT CODE .......................... 106

A.1.2 LOOP-LOOP OVER Dipole TARGETS .................................................................. 110 


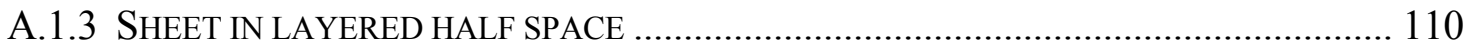

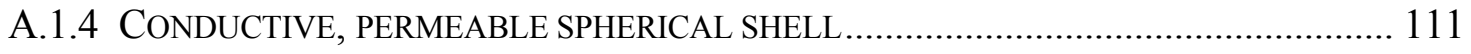

A.1.5 3-D HALF-SPACE OF ARBITRARY CONDUCTIVITY AND PERMEABILITY.................. 113 


\section{INTRODUCTION}

\subsection{Background}

In the search for UXO and for discrimination between UXO and non-UXO metallic fragments (clutter) it is necessary to accurately determine the parameters that characterize a metallic object in the ground. A search system is needed that not only detects the object itself but can also determine its size, shape, orientation, shell thickness and metal content (ferrous or non-ferrous, mixed metals). These properties of a buried metallic object are referred to here as the object parameters.

The search for UXO is a two-step process. The object must first be detected and its location determined then the parameters of the object must be defined. The first step is now accomplished with a variety of magnetometer and active electromagnetic (AEM) systems. The AEM systems operate in the transient or frequency domain mode and at present use a single transmitter and up to three receivers. The characterization phase can also be described as a two-step process. A variety of incident fields are used to induce magnetization and current flow in different directions within the object. The magnetic dipole moments induced in the body, normalized by the inducing field are known as the polarizabilities of the object. The secondary fields related to these induced polarizabilities, as a function of frequency or time, are the measured quantities. Interpretation could stop with the determination of the object location and the three orthogonal principal polarizabilities. These polarizabilities and their variation with either time or frequency are the only fundamental object parameters that can be recovered from the inductive excitation

of a finite body in the ground if a dipolar representation is assumed. Presumably a catalog of polarizabilities could be constructed from the forward model polarizabilities for a wide range of potential targets and then a look-up table inversion scheme could be used to identify the actual dimensions and physical properties of a target.

We have found that a powerful second step in the characterization process is to directly interpret the polarizabilities in terms of the principal axes of an equivalent spheroid. This step yields the size and aspect ratio of the spheroid that is physically equivalent to the target. Finally, the wideband target response permits the estimation of 
both the permeability-conductivity ratio and the conductivity-permeability product so that each can be estimated independently. It is unlikely that any better resolution of the shape of an intact UXO than its equivalent spheroid can be recovered using practical system data. A catalog of equivalent spheroidal bodies representing most UXOs would thus be relatively easy to construct for final target identification. This more detailed second step requires the broadband spectral or transient response using frequencies low enough to identify the quasi dc magnetization response and high enough to identify the purely electromagnetic (EM), inductive limit, response which depends only on the size of the object.

The concept of characterizing UXO by equivalent spheroid parameters is key to distinguishing intact UXO from non-UXO metal scrap. Any UXO is expected to retain its fundamental shape (size, aspect ratio and symmetry about its long axis) with perhaps minor distortion caused by impact. Metal scrap will have distinct polarizability signatures that cannot mimic these of elongated symmetric bodies. Roughly flat sheets will have dipolar responses approaching these of a highly flattened oblate spheroid (close to a loop response), twisted sheets a principal moment orthogonal to some equivalent plane through the sheet with small and highly irregular minor axis polarizabilities etc. These distinguishing polarizabilities, coupled with the size estimates and spatial sampling of the multiple receiver array are more than enough to separate small scrap from deeper targets of concern.

An optimal EM system can extract from the measurements the best possible estimates of the location, orientation, size, shape and metal content of a buried metallic object in the presence of the interfering response of the ground and non-UXO metallic objects. Discrimination can be achieved partly by selective filtering of the responses inherent in the system configuration and design and partly through post acquisition processing. The target parameters are obtained by a statistical inversion of the measurements to establish the principal electromagnetic moments of the detected object and their variation with time or frequency. 


\subsection{Objectives}

In summary the technical objectives of this project were:

1) To develop and demonstrate a methodology for the quantitative evaluation of existing AEM systems and for the design of new systems.

2) To implement a new methodology for optimizing an AEM system for detecting and classifying UXO of a given class in a specified geologic setting and in a given noise environment.

3) To design and build a prototype of an active EM system for detecting and characterizing a metallic object in the ground.

\subsection{Technical Approach}

The design methodology is based on the use of simulators, numerical models of the electromagnetic response of an arbitrary target in the ground to an arbitrary configuration of transmitters and receivers. The variables in the simulator are the parameters of the targets, the parameters of the AEM system and the noise (ambient, motion, instrumental, and geologic). The target parameters are the location, orientation, depth, size, shape, metal content and type. A schematic of the simulator is shown in Figure 1.3.1.

\section{EVALUATION}

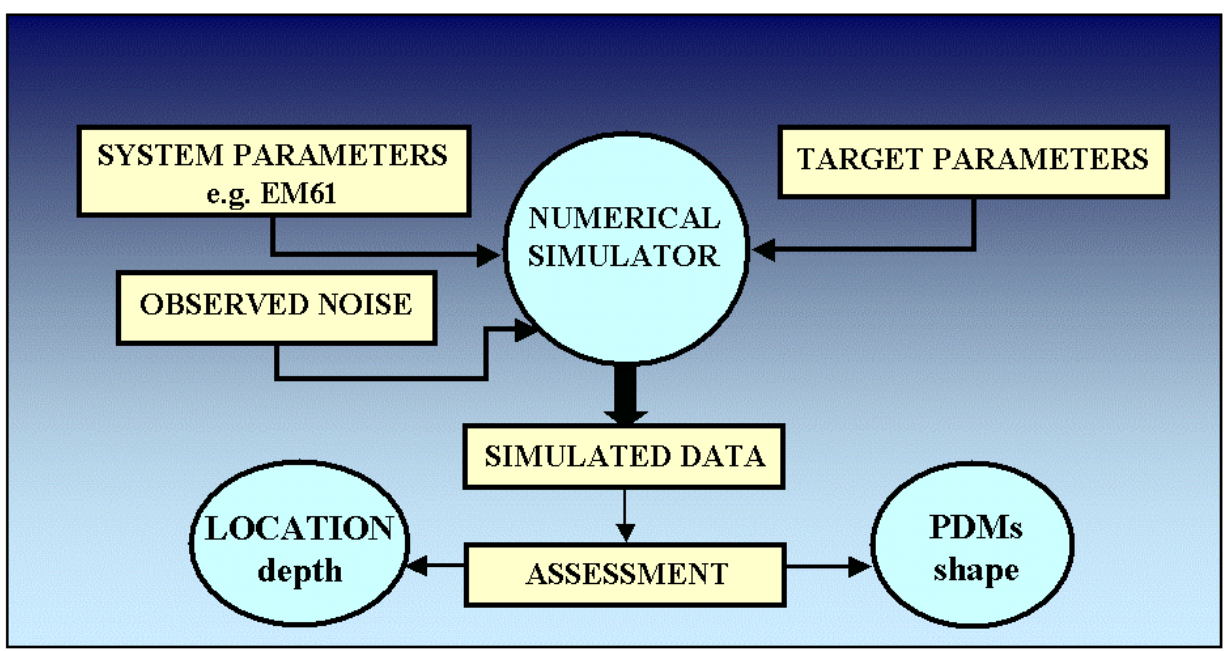

Figure 1.3.1: Schematic diagram of the simulator. 


\section{OPTIMIZATION}

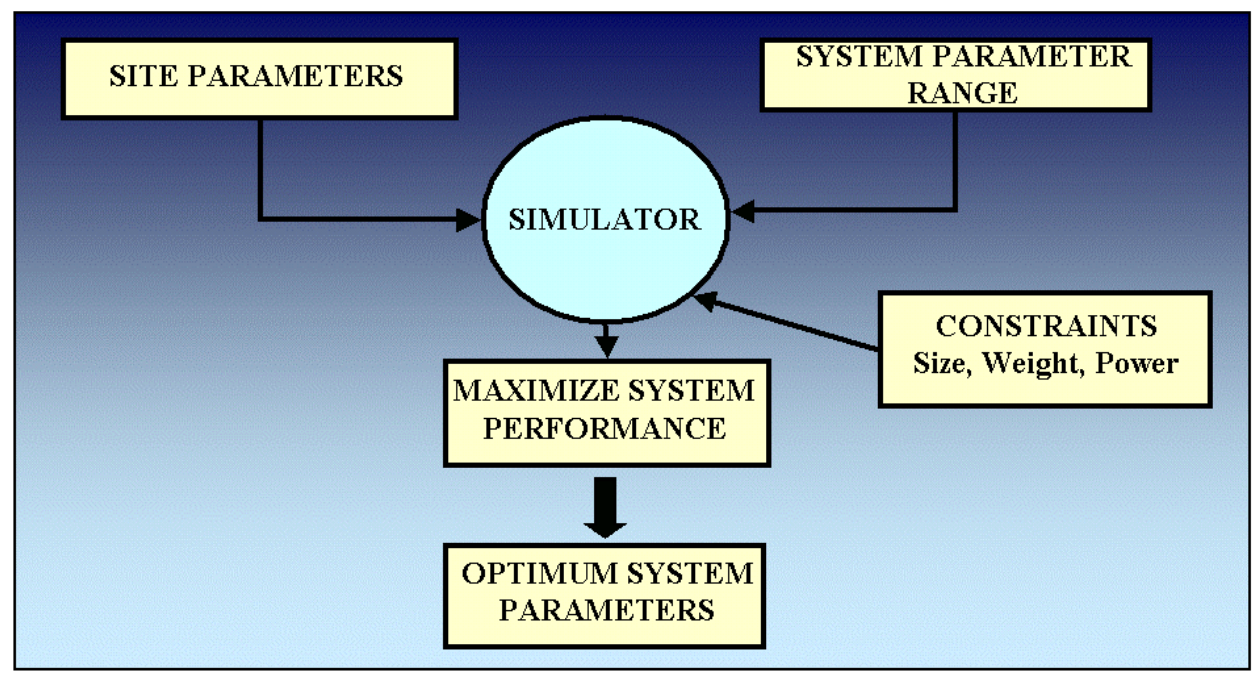

Figure 1.3.2: Schematic diagram of the optimization process.

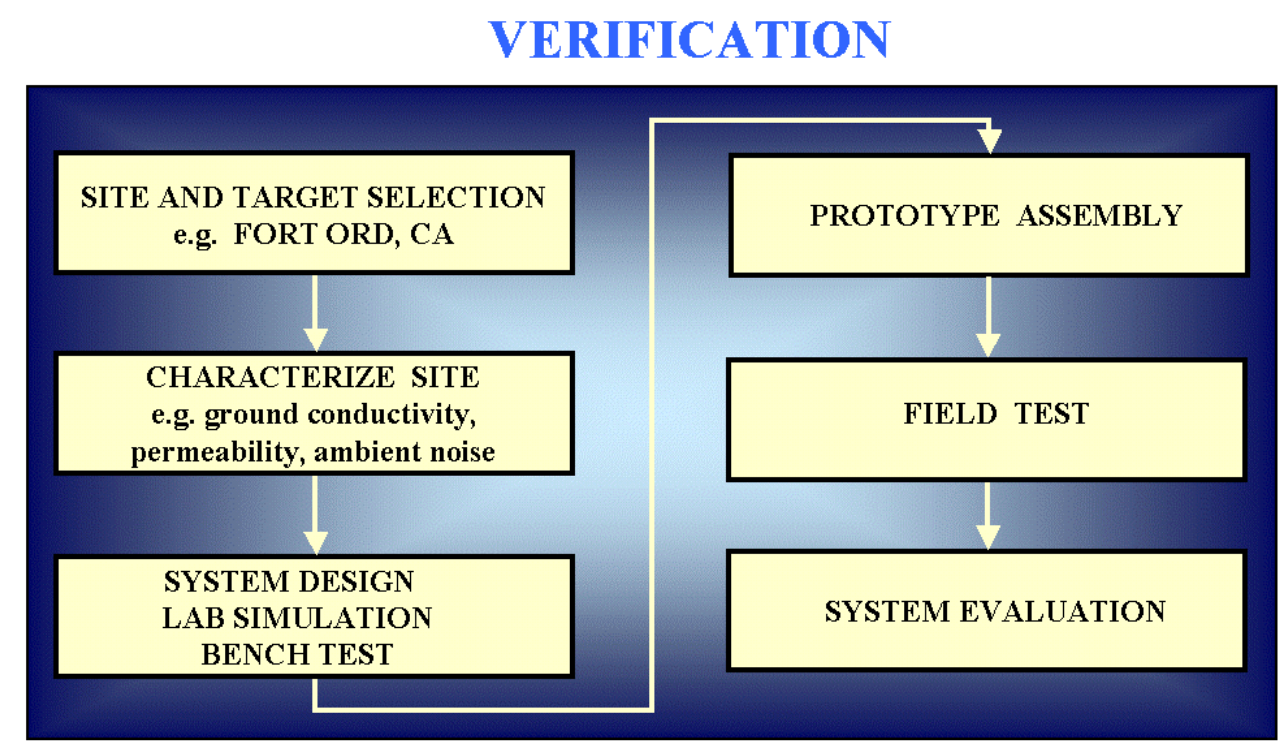

Figure 1.3.3: Schematic diagram of the verification process.

The simulator can be used to evaluate designs by simulating the response of a given system, with a given noise level, to a particular target. Various system configurations can 
be quantitatively compared through their respective signal to noise ratios over the same target.

This forward modeling approach is useful for evaluating the relative response of different targets for a given system, for example in analyzing the role of loop size in discriminating between small, shallow, targets and deeper, larger, ones. It is also useful for evaluating the ground response and modeling the spectral or transient response of various targets.

The system parameters that are variables in the design are: a) the geometric configuration of the transmitter(s) and receivers(s) and the way in which they are mounted for given target objectives (the platform), b) the spatial positioning of the system (profile, grid, single site stand-off, etc.), c) the transmitter power and waveform, d) the system and ambient noise, e) the receiver bandwidth and dynamic range, $f$ ) the signal averaging time (a function of survey speed). The geologic variables (geologic noise) are the values and variability of the ground conductivity and permeability. A more detailed schematic of all the variables that contribute to the data acquired with a general AEM system is shown in Figure 1.3.4.

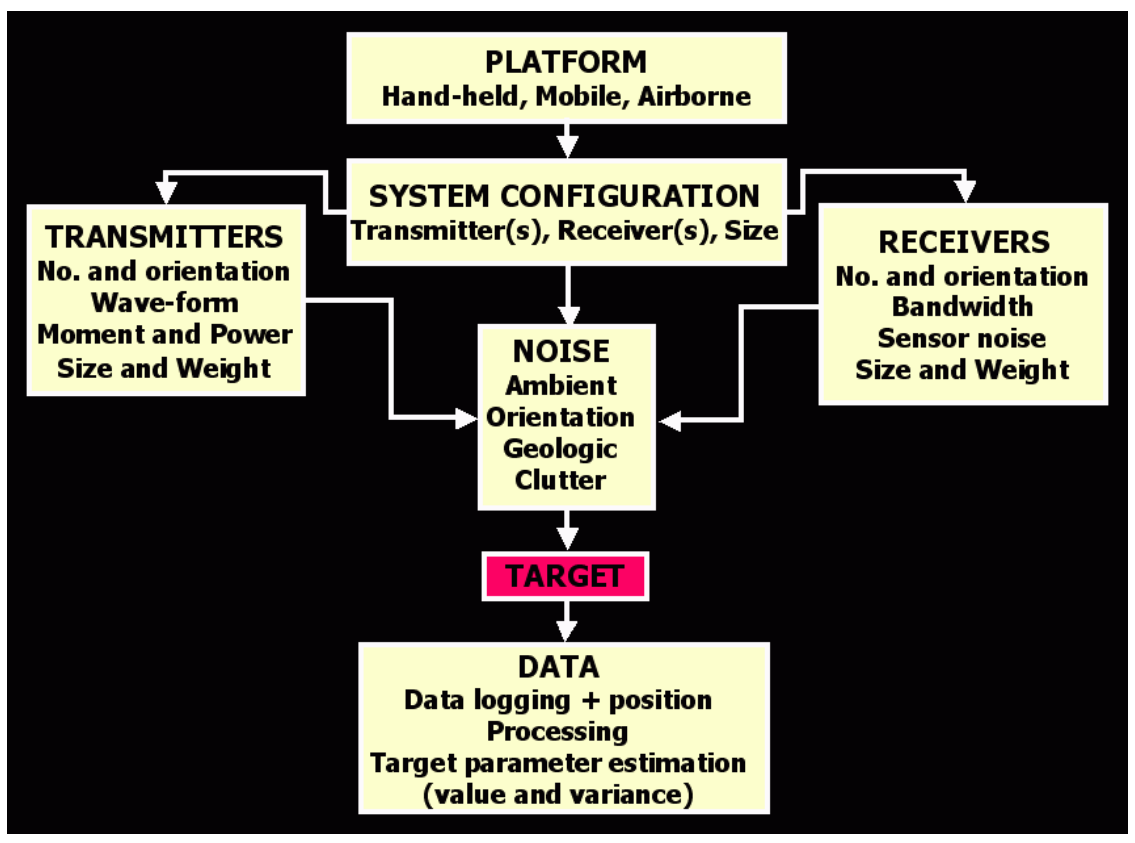

Figure 1.3.4: Generic AEM System. 
In inverse mode the simulator is used to determine target properties from measurements with a given transmitter-receiver configuration. At any given time in the transient response (or at a given frequency in the frequency domain) the response data are inverted to yield the location $(\mathrm{x}, \mathrm{y}, \mathrm{z})$ of the target, its attitude and its principal polarizabilities (yielding an apparent aspect ratio). In this process noise estimates (or measurements) are interpreted to yield error estimates of the object location, attitude and polarizabilities. This inversion, at a succession of times in the transient, or at many frequencies, yields the object polarizabilities as a function of time or frequency, which can in turn yield the size, true aspect ratio and estimates of the conductivity and permeability of the target. The accuracy of these property estimates depends on the time or frequency window over which the polarizability measurements, and their accuracies, are known. This particular inversion process can be used on artificial test data to determine the bandwidth of the system that is needed to provide the desired accuracy in the physical property measurement.

Finally, the simulator was used to determine the optimum configuration for detecting and characterizing the target. In this step certain transmitter configurations are selected and the position and orientation of $\mathrm{n}$ receivers (with given signal-to-noise ratios) are varied until targets of various shape and depth are characterized. The array is judged as optimum when the errors in target parameter estimation are minimized. An initial analysis using this approach for targets of specific polarizability led to the conclusion that a system employing three orthogonal transmitters and a minimum of five vertical receivers deployed within the footprint of the horizontal loop transmitter would be optimal. In this analysis, the criterion for optimization was that the sum of the squared error in the individual principal polarizability be a minimum. This left open the possibility that certain configurations might be used to estimate one polarizability very well while yielding poor estimates of the other two.

Since the accurate determination of the polarizabilities is needed to determine the true aspect ratio and physical properties of the target, the inversion algorithm was extended to determine the number of receivers and their orientation needed to resolve polarizabilities of any arbitrary target located beneath the transmitter-receiver array. This analysis showed that more than five receivers would be advantageous but that using multiple receiver 
orientations had little practical advantage over slightly larger arrays of only vertical receivers.

The results of all these quantitative analyses led to the preliminary design of a configuration using three orthogonal loop transmitters and eight vertical receivers deployed in an asymmetric pattern in the plane of the horizontal loop. This is shown schematically in Figure 1.3.5 and a photo of the bench prototype, showing one of the loop transmitters, is shown in Figure 1.3.6.

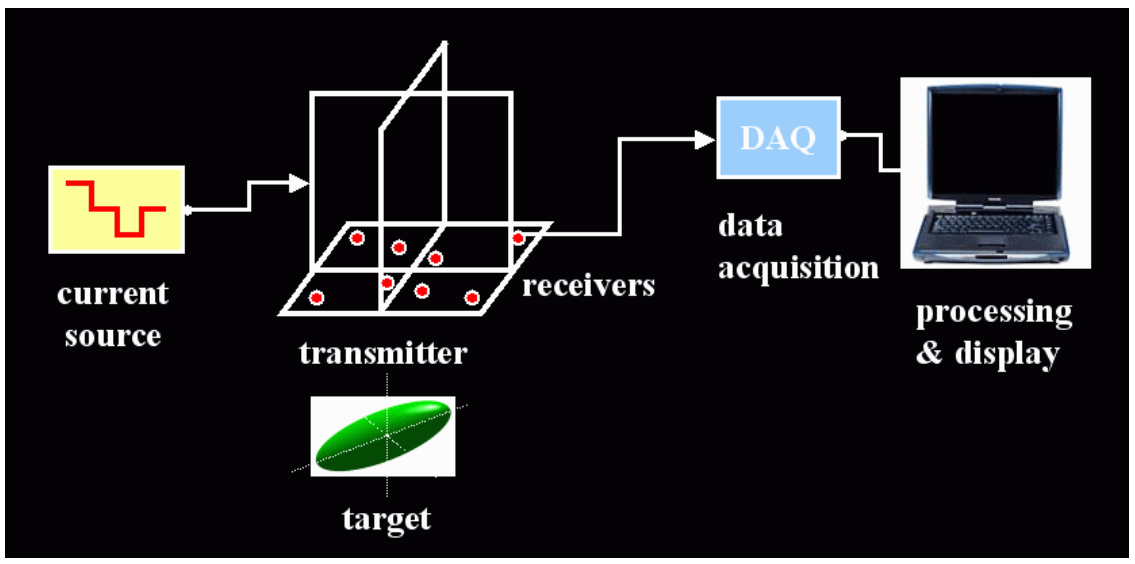

Figure 1.3.5: Schematics of the bench test acquisition setup.

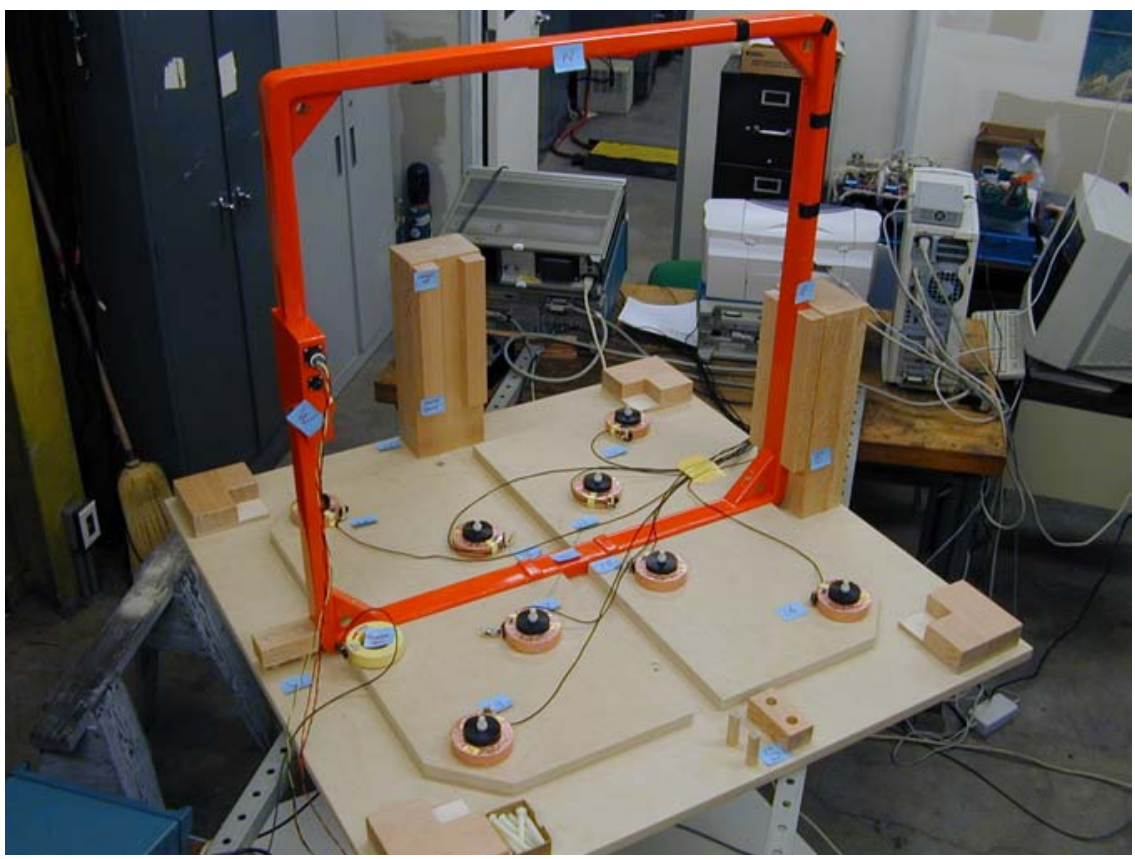

Figure 1.3.6: Photo of the prototype system. 


\section{INVERSION FOR TARGET PARAMETERS AND THE ROLE OF SYSTEM CONFIGURATION}

\subsection{Estimating Equivalent Dipole Polarizabilities for the Inductive Response of Isolated Conductive Bodies}

\subsubsection{Introduction}

Any set of currents can be characterized in terms of a set of their multipole moments. The associated magnetic fields can be represented as a sum of corresponding multipole terms away from the currents (e.g., Jackson, 1975, p.746). For a magnetic multipole term of order $n$, magnetic field strengths fall off as $1 / \mathrm{r}^{\mathrm{n}+2}$, in resistive media. Dipole terms are the lowest order magnetic multipole terms. At distances much greater than the scale of an object, dipole terms become a very good approximation to the magnetic fields arising from currents induced in the object.

In the vicinity of a conductive body, the primary magnetic field imposed by an external source current may be approximated by the primary magnetic field at the object center $\mathbf{r}_{0}, \mathbf{B}^{(\mathrm{p})}\left(\mathbf{r}_{0}, \mathrm{t}\right)$. Assuming a common time variation $\mathrm{g}(\mathrm{t})$ for all primary magnetic field components at the object center, we define a primary field magnitude vector as $\mathbf{B}^{(0)} \equiv \mathbf{B}^{(\mathrm{p})}\left(\mathbf{r}_{0}, \mathrm{t}\right) / \mathrm{g}(\mathrm{t})$. We choose the normalization of $\mathrm{g}(\mathrm{t})$ so that $\mathrm{g}\left(\mathrm{t}_{0}\right)=1$ at some chosen time $t_{0}$, for example, for a step function turn-off primary field we choose the scale of $g(t)$ so that $\mathrm{g}(\mathrm{t})=1$ for $\mathrm{t}<0$. In practice, it is common to assume that the medium surrounding the object is sufficiently resistive that the magnetic fields due to currents induced in the surrounding medium are negligible at the body, so that $\mathrm{g}(\mathrm{t})$ is simply the transmitter current waveform. Neglecting primary field gradients, the secondary magnetic fields

$$
\mathbf{B}^{(\mathrm{s})}(\mathbf{r}, \mathrm{t}) \equiv \mathbf{B}(\mathbf{r}, \mathrm{t})-\mathbf{B}^{(\mathrm{p})}(\mathbf{r}, \mathrm{t})
$$

due to currents induced in a conductive body can be written as linear combinations of the magnetic fields that would be induced by primary fields of strength $g(t)$ in the $\hat{\boldsymbol{x}}, \hat{\boldsymbol{y}}$, or $\hat{\boldsymbol{z}}$ direction at the objects center, $\mathbf{B}_{\mathrm{x}}{ }^{(\mathrm{s})}(\mathbf{r}, \mathrm{t}), \mathbf{B}_{\mathrm{y}}{ }^{(\mathrm{s})}(\mathbf{r}, \mathrm{t})$, or $\mathbf{B}_{\mathrm{z}}{ }^{(\mathrm{s})}(\mathbf{r}, \mathrm{t})$ respectively;

$$
\mathbf{B}^{(\mathrm{s})}(\mathbf{r}, \mathrm{t})=\mathrm{B}_{\mathrm{x}}{ }^{(0)} \mathbf{B}_{\mathrm{x}}{ }^{(\mathrm{s})}(\mathbf{r}, \mathrm{t})+\mathrm{B}_{\mathrm{y}}{ }^{(0)} \mathbf{B}_{\mathrm{y}}{ }^{(\mathrm{s})}(\mathbf{r}, \mathrm{t})+\mathrm{B}_{\mathrm{z}}{ }^{(0)} \mathbf{B}_{\mathrm{z}}{ }^{(\mathrm{s})}(\mathbf{r}, \mathrm{t}),
$$

where $\mathrm{B}_{\mathrm{x}}{ }^{(0)}, \mathrm{B}_{\mathrm{y}}{ }^{(0)}, \mathrm{B}_{\mathrm{z}}{ }^{(0)}$ are the $\mathrm{x}, \mathrm{y}$, and $\mathrm{z}$ components of $\mathbf{B}^{(0)}$. Being secondary magnetic fields induced by a source magnetic field of strength $g(t)$ (which is dimensionless) 
$\mathbf{B}_{\mathrm{x}}{ }^{(\mathrm{s})}(\mathbf{r}, \mathrm{t}), \mathbf{B}_{\mathrm{y}}{ }^{(\mathrm{s})}(\mathbf{r}, \mathrm{t})$, and $\mathbf{B}_{\mathrm{z}}{ }^{(\mathrm{s})}(\mathbf{r}, \mathrm{t})$ are similarly dimensionless. Forming a matrix with the three vectors $\mathbf{B}_{\mathrm{x}}{ }^{(\mathrm{s})}(\mathbf{r}, \mathrm{t}), \mathbf{B}_{\mathrm{y}}{ }^{(\mathrm{s})}(\mathbf{r}, \mathrm{t})$, and $\mathbf{B}_{\mathrm{z}}{ }^{(\mathrm{s})}(\mathbf{r}, \mathrm{t})$ as its columns, the right hand side of Equation 2.1.2 is a matrix vector product;

$$
\mathbf{B}^{(\mathrm{s})}(\mathbf{r}, \mathrm{t})=\left[\mathbf{B}_{\mathrm{x}}{ }^{(\mathrm{s})}(\mathbf{r}, \mathrm{t}), \mathbf{B}_{\mathrm{y}}{ }^{(\mathrm{s})}(\mathbf{r}, \mathrm{t}), \mathbf{B}_{\mathrm{z}}^{(\mathrm{s})}(\mathbf{r}, \mathrm{t})\right] \cdot \mathbf{B}^{(0)},
$$

where $\mathbf{B}^{(\mathrm{s})}(\mathbf{r}, \mathrm{t}), \mathbf{B}_{\mathrm{x}}{ }^{(\mathrm{s})}(\mathbf{r}, \mathrm{t}), \mathbf{B}_{\mathrm{y}}^{(\mathrm{s})}(\mathbf{r}, \mathrm{t}), \mathbf{B}_{\mathrm{z}}{ }^{(\mathrm{s})}(\mathbf{r}, \mathrm{t})$ and $\mathbf{B}^{(0)}$ considered as column vectors, the dot effect matrix multiplication.

At distances where non-dipole secondary magnetic fields are small, the secondary magnetic fields induced by the primary magnetic field in the $\hat{\boldsymbol{x}}$ direction can be broken into contributions by dipole components in the $\mathrm{x}, \mathrm{y}$, and $\mathrm{z}$ directions;

$$
\mathbf{B}_{\mathrm{x}}{ }^{(\mathrm{s})}(\mathbf{r}, \mathrm{t})=\mathrm{m}_{\mathrm{xx}}(\mathrm{t}) \mathbf{B}_{\mathrm{x}}{ }^{(\mathrm{d})}(\mathbf{r})+\mathrm{m}_{\mathrm{yx}}(\mathrm{t}) \mathbf{B}_{\mathrm{y}}{ }^{(\mathrm{d})}(\mathbf{r})+\mathrm{m}_{\mathrm{zx}}(\mathrm{t}) \mathbf{B}_{\mathrm{z}}{ }^{(\mathrm{d})}(\mathbf{r}),
$$

where $\mathbf{B}_{\mathrm{x}}{ }^{(\mathrm{d})}(\mathbf{r}), \mathbf{B}_{\mathrm{y}}{ }^{(\mathrm{d})}(\mathbf{r})$, and $\mathbf{B}_{\mathrm{z}}{ }^{(\mathrm{d})}(\mathbf{r})$ are the magnetic fields of a unit magnetic dipole in the $\hat{\boldsymbol{x}}, \hat{\boldsymbol{y}}$, and $\hat{\boldsymbol{z}}$ directions respectively, placed at the body center, and have units of Tesla/Amp- $m^{2}$. Quantities $m_{x x}(t), m_{y x}(t)$, and $m_{z x}(t)$ are the current dipole moments in these directions, for a unit primary (inducing) magnetic field in the $\hat{\boldsymbol{x}}$ direction at the object center, and have units of Amp- $\mathrm{m}^{2} /$ Tesla. Similarly,

$$
\begin{aligned}
& \mathbf{B}_{\mathrm{y}}{ }^{(\mathrm{s})}(\mathbf{r}, \mathrm{t})=\mathrm{m}_{\mathrm{xy}}(\mathrm{t}) \mathbf{B}_{\mathrm{x}}{ }^{(\mathrm{d})}(\mathbf{r})+\mathrm{m}_{\mathrm{yy}}(\mathrm{t}) \mathbf{B}_{\mathrm{y}}{ }^{(\mathrm{d})}(\mathbf{r})+\mathrm{m}_{\mathrm{zy}}(\mathrm{t}) \mathbf{B}_{\mathrm{z}}{ }^{(\mathrm{d})}(\mathbf{r}), \\
& \mathbf{B}_{\mathrm{z}}{ }^{(\mathrm{s})}(\mathbf{r}, \mathrm{t})=\mathrm{m}_{\mathrm{xz}}(\mathrm{t}) \mathbf{B}_{\mathrm{x}}{ }^{(\mathrm{d})}(\mathbf{r})+\mathrm{m}_{\mathrm{yz}}(\mathrm{t}) \mathbf{B}_{\mathrm{y}}{ }^{(\mathrm{d})}(\mathbf{r})+\mathrm{m}_{\mathrm{zz}}(\mathrm{t}) \mathbf{B}_{\mathrm{z}}{ }^{(\mathrm{d})}(\mathbf{r}),
\end{aligned}
$$

with $m_{x y}(t), m_{y y}(t), m_{z y}(t)$ and $m_{x z}(t), m_{y z}(t), m_{z z}(t)$ the corresponding moments for primary magnetic fields in the $\hat{\boldsymbol{y}}$ and $\hat{\boldsymbol{z}}$ directions. Assuming that the surrounding medium is sufficiently resistive that tertiary currents induced in the surrounding medium by the magnetic fields due to currents in the body can be neglected, the effective magnetic dipole moments correspond to the actual moments of the currents circulating in the body. We make this assumption, and henceforth refer to them simply as the dipole moments. Equations (2.1.4) can be written in matrix form as

$$
\left[\mathbf{B}_{\mathrm{x}}{ }^{(\mathrm{s})}(\mathbf{r}), \mathbf{B}_{\mathrm{y}}{ }^{(\mathrm{s})}(\mathbf{r}), \mathbf{B}_{\mathrm{z}}{ }^{(\mathrm{s})}(\mathbf{r})\right]=\left[\mathbf{B}_{\mathrm{x}}{ }^{(\mathrm{d})}(\mathbf{r}), \mathbf{B}_{\mathrm{y}}{ }^{(\mathrm{d})}(\mathbf{r}), \mathbf{B}_{z}^{(\mathrm{d})}(\mathbf{r})\right]\left[\begin{array}{l}
\mathrm{m}_{\mathrm{xx}} \mathrm{m}_{\mathrm{xy}} \mathrm{m}_{\mathrm{xz}} \\
\mathrm{m}_{\mathrm{yx}} \mathrm{m}_{\mathrm{yy}} \mathrm{m}_{\mathrm{yz}} \\
\mathrm{m}_{\mathrm{zx}} \mathrm{m}_{\mathrm{zy}} \mathrm{m}_{\mathrm{zz}}
\end{array}\right]
$$

where the explicit time dependence of the matrix of dipole moments has been omitted.

The matrix of dipole moments $\mathbf{M}$ is symmetric (Landau and Lifshitz, 1960, p192).

Substituting equation (2.1.5) into equation (2.1.3) gives 


$$
\mathbf{B}^{(\mathrm{s})}(\mathbf{r}, \mathrm{t})=\left[\mathbf{B}_{\mathrm{x}}^{(\mathrm{d})}(\mathbf{r}), \mathbf{B}_{\mathrm{y}}^{(\mathrm{d})}(\mathbf{r}), \mathbf{B}_{\mathrm{z}}^{(\mathrm{d})}(\mathbf{r})\right] \mathbf{M}(\mathrm{t}) \mathbf{B}^{(0)}
$$

In time domain applications, $\mathbf{M}$ is real, in addition to being symmetric, so can be diagonalized by an orthogonal matrix $\mathbf{U}(\mathrm{t})$;

$$
\mathbf{L}(\mathrm{t})=\mathbf{U}^{\mathrm{T}}(\mathrm{t}) \mathbf{M}(\mathrm{t}) \mathbf{U}(\mathrm{t})
$$

where ${ }^{\mathrm{T}}$ denotes transpose, $\mathbf{L}(\mathrm{t})$ is diagonal, with elements $\mathrm{L}_{11}(\mathrm{t}), \mathrm{L}_{22}(\mathrm{t}), \mathrm{L}_{33}(\mathrm{t})$ known as the principal moments of $\mathbf{M}(\mathrm{t})$, and have the same units as $\mathbf{M}\left(\mathrm{Amp}-\mathrm{m}^{2} /\right.$ Tesla). Equation (2.1.7a) expresses $\mathbf{M}(\mathrm{t})$ in coordinates given by the columns of $\mathbf{U}(\mathrm{t}),\left(\mathbf{u}_{\mathrm{i}}\right)$, known as the principal directions of $\mathbf{M}(\mathrm{t})$. For bodies with an axis of symmetry $\widehat{\mathbf{w}}, \widehat{\mathbf{w}}$ is one principal direction (e.g., $\mathbf{u}_{1}$ ), with corresponding principal component (e.g., $\mathrm{L}_{11}$ ) giving the equivalent dipole moment induced in the $\widehat{\mathbf{w}}$ direction for a unit primary field in the $\widehat{\mathbf{w}}$ direction at the object center. The other two principal moments correspond to equivalent dipole moments induced in directions normal to $\widehat{\mathbf{w}}$ for unit primary fields in those directions. Symmetry of the object implies that the latter two moments are equal. For a symmetric object, rotating into coordinates aligned with the object's symmetry axis diagonalizes $\mathbf{M}$, so may be accomplished by a rotation matrix $\mathbf{U}$, which is independent of time.

This definition of the equivalent dipole polarizability matrix $\mathbf{M}$ is consistent with that used by Pasion and Oldenburg (2001), and differs by a factor of $\mu_{0}$ from that used by Baum (1999).

As written, equation (2.1.6) represents the magnetic field $\mathbf{B}^{(0)}(\mathbf{r}, \mathrm{t})$ as a linear combination of dipole fields. Differentiating it, one can apply the same methods to modeling measurements of $\mathrm{d} \mathbf{B}^{(\mathrm{s})}(\mathbf{r}, \mathrm{t}) / \mathrm{dt}$, with $\mathrm{d} \mathbf{M}(\mathrm{t}) / \mathrm{dt}$ replacing $\mathbf{M}(\mathrm{t})$. In this case, $\mathrm{d} \mathbf{M}(\mathrm{t}) / \mathrm{dt}$ may be diagonalized analogously to Equation (2.1.7a);

$$
\mathbf{L}^{\prime}(\mathrm{t})=\mathbf{U}^{\prime} \mathrm{T}(\mathrm{t}) \mathbf{M}(\mathrm{t}) \mathbf{U}^{\prime}(\mathrm{t})
$$

Where for a symmetric object, diagonalization may be accomplished with the same time dependent matrix as before; $\mathbf{U}^{\prime}(\mathrm{t})=\mathbf{U}$. Strictly speaking $d \mathbf{M} / \mathrm{dt}$ and $\mathbf{L}^{\prime}(\mathrm{t})$ represent equivalent dipole polarizability (decay) rates rather than equivalent dipole polarizabilities. In common usage, they are referred to simply as polarizabilities, but can be distinguished by having units of Amp- $\mathrm{m}^{2} / \mathrm{s}-$ Tesla rather than Amp- $\mathrm{m}^{2} /$ Tesla.

For a given time dependence of source, $g(t)$, equation (2.1.6) relates secondary fields at any time to an equivalent dipole polarizability $\mathbf{M}(\mathrm{t})$ for that time, so $\mathbf{M}(\mathrm{t})$ may be 
estimated separately for each time. Consequently, we drop the explicit time dependence, and assume that all measurements are at a single time relative to the starting time for the primary field pulse.

\subsubsection{Estimating Dipole Polarizabilities When Object Center is Known}

When the object center location is known, $\mathbf{B}^{(0)}$ can be calculated for each of a set of sources with the same time dependence $\mathrm{g}(\mathrm{t})$. For the $\mathrm{i}^{\prime}$ th measurement of a set of $n$ measurements, letting $\mathbf{B}_{i}^{(0)}$ be the primary field polarization vector at the object center, $\mathbf{r}_{\mathrm{i}}$ be the location of a magnetic field measurement, and $\hat{\mathbf{v}}_{i}$ be the orientation of the magnetic field receiver (e.g., coil), equation (2.1.6) written for the $\hat{\mathbf{v}}_{\mathbf{i}}$ component at $\mathbf{r}_{\mathrm{i}}$ is

$$
\hat{\mathbf{v}}_{\mathrm{i}}^{\mathrm{T}} \mathbf{B}^{(\mathrm{s})}\left(\mathbf{r}_{\mathrm{i}}\right)=\hat{\mathbf{v}}_{\mathrm{i}}^{\mathrm{T}} \cdot\left[\mathbf{B}_{\mathrm{x}}{ }^{(\mathrm{d})}\left(\mathbf{r}_{\mathrm{i}}\right), \mathbf{B}_{\mathrm{y}}{ }^{(\mathrm{d})}\left(\mathbf{r}_{\mathrm{i}}\right), \mathbf{B}_{\mathrm{z}}{ }^{(\mathrm{d})}\left(\mathbf{r}_{\mathrm{i}}\right)\right] \mathbf{M} \mathbf{B}_{\mathrm{i}}{ }^{(0)},
$$

one (scalar) equation constraining the six unknown dipole polarizabilities $\mathrm{m}_{\mathrm{xx}}, \mathrm{m}_{\mathrm{yy}}, \mathrm{m}_{\mathrm{zz}}$, $\mathrm{m}_{\mathrm{xy}}=\mathrm{m}_{\mathrm{yx}}, \mathrm{m}_{\mathrm{yz}}=\mathrm{m}_{\mathrm{zy}}$, and $\mathrm{m}_{\mathrm{xz}}=\mathrm{m}_{\mathrm{zx}}$, for each receiver source combination. This can be rewritten as

$$
d_{i}=f_{i x x} m_{x x}+f_{i y y} m_{y y}+f_{i z z} m_{z z}+f_{i x y} m_{x y}+f_{i y z} m_{y z}+f_{i x z} m_{x z},
$$

where $\mathrm{d}_{\mathrm{i}} \equiv \hat{\mathbf{v}}_{\mathrm{i}}^{\mathrm{T}} \mathbf{B}^{(\mathrm{s})}\left(\mathbf{r}_{\mathrm{i}}\right)$, and the coefficients $\mathrm{f}_{\mathrm{i} x \mathrm{xx}}, \mathrm{f}_{\mathrm{i} \text { yy }}, \ldots$, can be found by multiplying out the vector and matrix products on the right side of equation (2.1.8), substituting $\mathrm{m}_{\mathrm{xy}}$, $\mathrm{m}_{\mathrm{yz}}$, and $\mathrm{m}_{\mathrm{xz}}$ for $\mathrm{m}_{\mathrm{yx}}, \mathrm{m}_{\mathrm{zy}}$, and $\mathrm{m}_{\mathrm{zx}}$, and identifying the coefficients of $\mathrm{m}_{\mathrm{xx}}, \mathrm{m}_{\mathrm{yy}}, \mathrm{m}_{\mathrm{zz}}, \mathrm{m}_{\mathrm{xy}}$, $\mathrm{m}_{\mathrm{yz}}$, and $\mathrm{m}_{\mathrm{xz}}$. Equations (2.1.9) can be written in matrix form as

$$
\mathbf{d}=\mathbf{F} \mathbf{m}
$$

where $\mathbf{m}=\left(\mathrm{m}_{\mathrm{xx}}, \mathrm{m}_{\mathrm{yy}}, \mathrm{m}_{\mathrm{zz}}, \mathrm{m}_{\mathrm{xy}}, \mathrm{m}_{\mathrm{yz}}, \mathrm{m}_{\mathrm{xz}}\right)^{\mathrm{T}}$, which has least squares solution

$$
\mathbf{m}=\left(\mathbf{F}^{\mathrm{T}} \mathbf{F}\right)^{-1} \mathbf{F}^{\mathrm{T}} \mathbf{d} \text {. }
$$

The dipole polarizability moment matrix $\mathbf{M}$ can be assembled from the elements of $\mathbf{m}$, using the symmetry of $\mathbf{M}$.

\subsubsection{Estimating Dipole Polarizabilities and Object Center Location}

When the object center position $\mathbf{r}_{0}$ is unknown, one may form equation (2.1.8) using dipole fields $\mathbf{B}_{\mathrm{x}}{ }^{(\mathrm{d})}, \mathbf{B}_{\mathrm{y}}{ }^{(\mathrm{d})}, \mathbf{B}_{\mathrm{z}}{ }^{(\mathrm{d})}$ calculated for dipoles centered at some candidate object center position $\mathbf{r}_{0}$, and primary field polarization vectors $\mathbf{B}_{i}^{(0)}$ at the candidate 
object center position, form equation (2.1.10), and calculate the least squares dipole polarizabilities $\mathbf{m}$ for that candidate center location, $\mathbf{m}\left(\mathbf{r}_{0}\right)$. Its squared misfit is

$$
\chi^{2} \equiv\left|\mathbf{d}-\hat{\mathbf{d}}\left(\mathbf{r}_{0}\right)\right|^{2} / \sigma^{2},
$$

where $\sigma^{2}$ is the squared measurement uncertainty, and

$$
\hat{\mathbf{d}}\left(\mathbf{r}_{0}\right) \equiv \mathbf{F}\left(\mathbf{F}^{\mathrm{T}} \mathbf{F}\right)^{-1} \mathbf{F}^{\mathrm{T}} \mathbf{d}
$$

is the best fitting data predicted for this choice of $\mathbf{r}_{0}$. Matrix $\mathbf{F}$ depends on $\mathbf{r}_{0}$ through $\mathbf{B}_{\mathrm{x}}$ (d) $, \mathbf{B}_{\mathbf{y}}{ }^{\left({ }^{(d)}\right.}, \mathbf{B}_{z}{ }^{(d)}$, and $\mathbf{B}_{\mathrm{i}}{ }^{(0)}$. We find the position $\mathbf{r}_{0}$ giving a minimum of squared misfit (2.1.12), using the downhill simplex algorithm (Press, et al., 1986, p. 289), started from four candidate center locations, $\mathbf{r}_{0}{ }^{(j)}, j=1, \ldots, 4$ at the corners of a tetrahedron with edges one quarter of the length of the maximum separation of receiver locations, centered one half the maximum receiver separation below the receivers. The downhill simplex algorithm moves the corners of the tetrahedron systematically expanding or contracting as necessary to arrive at a minimum of the minimized function, (the squared misfit), and ends when the corners have converged within a small tolerance of each other, or the function values at the four corners are within a small tolerance of each other.

\subsubsection{Estimating Dipole Polarizability Uncertainties}

For data with small measurement errors, the uncertainty in the resultant dipole polarizabilities and equivalent dipole position (object center) may be obtained from analysis of a linearized inversion for dipole polarizabilities and position. Denoting dipole components at the $i^{\prime}$ th receiver in the receiver direction $\mathbf{v}_{\mathrm{i}}$ by

$$
\mathrm{B}_{\mathrm{i} 1}^{\prime}{ }^{(\mathrm{d})} \equiv \hat{\mathbf{v}}_{\mathrm{u}}{ }^{\mathrm{T}} \mathbf{B}_{\mathrm{x}}{ }^{(\mathrm{d})}\left(\mathbf{r}_{\mathrm{i}}\right), \quad \mathrm{B}_{\mathrm{i} 2}^{\prime}{ }^{(\mathrm{d})} \equiv \hat{\mathbf{v}}_{\mathrm{u}}{ }^{\mathrm{T}} \mathbf{B}_{\mathrm{y}}{ }^{(\mathrm{d})}\left(\mathbf{r}_{\mathrm{i}}\right), \quad \mathrm{B}_{\mathrm{i} 3}^{(\mathrm{d})} \equiv \hat{\mathbf{v}}_{\mathrm{u}}{ }^{\mathrm{T}} \mathbf{B}_{\mathrm{z}}{ }^{(\mathrm{d})}\left(\mathbf{r}_{\mathrm{i}}\right)
$$

for $\mathrm{x}, \mathrm{y}$, and $\mathrm{z}$ dipoles respectively, denoting $\mathrm{x}, \mathrm{y}$, and $\mathrm{z}$ components of the center primary field $\mathbf{B}_{\mathrm{i}}{ }^{(0)}$ by $\mathrm{B}_{1 \mathrm{i}}{ }^{(0)}, \mathrm{B}_{2 \mathrm{i}}{ }^{(0)}$, and $\mathrm{B}_{3 \mathrm{i}}{ }^{(0)}$, and numbering the elements of $\mathbf{M}$ as $\mathrm{m}_{\mathrm{kj}}$, for $\mathrm{k}=1,3$, $\mathrm{j}=1,3$, then equation (2.1.8) can be written explicitly as

$$
\mathrm{d}_{\mathrm{i}}=\sum_{\mathrm{k}=1}^{3} \sum_{\mathrm{j}=1}^{3} \mathrm{~B}_{\mathrm{ik}}^{\prime(d)} \mathrm{B}_{\mathrm{ji}}{ }^{(0)} \mathrm{m}_{\mathrm{kj}} .
$$

When equivalent dipole position $\mathbf{r}_{0}$ is not known a priori, one can expand equation (2.15) in a Taylor series about an initial value $\mathbf{r}_{0}^{(\mathrm{q})}$, such as the result of the downhill simplex method search of the previous section. Letting $\mathbf{M}^{(\mathfrak{q})}$ be the corresponding dipole 
polarizability matrix fit for candidate dipole position $\mathbf{r}_{0}^{(\mathrm{q})}$, a first order Taylor expansion about $\mathbf{r}_{0}^{(\mathrm{q})}$ yields

$$
\mathrm{d}_{\mathrm{i}}=\sum_{\mathrm{k}=1}^{3} \sum_{\mathrm{j}=1}^{3} \mathrm{~B}_{\mathrm{ik}}{ }^{(\mathrm{d})} \mathrm{B}_{\mathrm{ji}}{ }^{(0)} \mathrm{m}_{\mathrm{kj}}{ }^{(\mathrm{q}+1)}+\mathrm{m}_{\mathrm{kj}}{ }^{(\mathrm{q})}\left[\mathbf{r}_{0}{ }^{(\mathrm{q}+1)}-\mathbf{r}_{0}{ }^{(\mathrm{q})}\right]^{\mathrm{T}} \cdot \nabla_{\mathbf{r}_{0}}\left[\mathrm{~B}_{\mathrm{ik}}{ }^{(\mathrm{d})} \mathrm{B}_{\mathrm{ji}}{ }^{(0)}\right] .
$$

Collecting coefficients of the new polarizability estimates $\mathrm{m}_{11}{ }^{(\mathrm{q}+1)}, \mathrm{m}_{22}{ }^{(\mathrm{q}+1)}, \ldots$, into a row vector $\mathbf{a}_{\mathrm{i}}{ }^{(\mathrm{q})}$, and coefficients of the components of change vector $\Delta \mathbf{r}_{0} \equiv \mathbf{r}_{0}{ }^{(\mathrm{q}+1)}-\mathbf{r}_{0}{ }^{(\mathrm{q})}$ into a row vector $\mathbf{g}_{\mathbf{j}}^{(\mathrm{q})}$ equation (2.1.16) becomes

$$
\mathrm{d}_{\mathrm{i}}=\left(\mathbf{a}_{\mathrm{i}}{ }^{(\mathrm{q})}, \mathbf{g}_{\mathrm{i}}^{(\mathrm{q})}\right)\left(\mathrm{m}_{11}, \mathrm{~m}_{22}, \mathrm{~m}_{33}, \mathrm{~m}_{12}, \mathrm{~m}_{23}, \mathrm{~m}_{13}, \Delta \mathrm{x}_{0}, \Delta \mathrm{y}_{0}, \Delta \mathrm{z}_{0}\right)^{\mathrm{T}},
$$

where superscript $(q+1)$ has been omitted from the various $\mathrm{m}_{\mathrm{ij}}{ }^{(\mathrm{q}+1)}$, and the symmetry of $\mathbf{M}$ has been used to eliminate $\mathrm{m}_{21}{ }^{(\mathrm{q}+1)}, \mathrm{m}_{32}{ }^{(\mathrm{q}+1)}$, and $\mathrm{m}_{31}{ }^{(\mathrm{q}+1)}$. This can be written in matrix form as

$$
\mathbf{d}=\widetilde{\mathbf{F}} \widetilde{\mathbf{m}}
$$

with the rows of $\widetilde{\mathbf{F}}$ and vector $\widetilde{\mathbf{m}}$ are the vectors on the right side of equation (2.17), and solved for $\tilde{\mathbf{m}}$ in the same manner as equations (2.1.10) and (2.1.11). A new estimated object center position is given by

$$
\mathbf{r}_{0}^{(\mathrm{q}+1)}=\mathbf{r}_{0}^{(\mathrm{q})}+\Delta \mathbf{r}_{0}
$$

Taylor expanding about the new estimate $\mathbf{r}_{0}{ }^{(\mathrm{q}+1)}$ (equation 2.1.16, with $\mathrm{q}$ incremented), the process is repeated until the change magnitude $\left|\Delta \mathbf{r}_{0}\right|$ is less than a small tolerance. The variances of the resultant dipole polarizabilities $\mathrm{m}_{\mathrm{xx}}, \mathrm{m}_{\mathrm{yy}}, \mathrm{m}_{\mathrm{zz}}, \mathrm{m}_{\mathrm{xy}}, \mathrm{m}_{\mathrm{yz}}, \mathrm{m}_{\mathrm{xz}}$, and equivalent dipole coordinates $\mathrm{x}_{0}, \mathrm{y}_{0}$, and $\mathrm{z}_{0}$, are given by the diagonal elements of the covariance matrix

$$
\operatorname{cov}(\tilde{\mathbf{m}})=\left(\tilde{\mathbf{F}}^{\mathrm{T}} \tilde{\mathbf{F}}\right)^{-1} \tilde{\mathbf{F}}^{\mathrm{T}} \operatorname{cov}(\mathbf{d}) \tilde{\mathbf{F}}\left(\tilde{\mathbf{F}}^{\mathrm{T}} \tilde{\mathbf{F}}\right)^{-1}
$$

For magnetic field measurements with squared uncertainty $\sigma^{2}$ and noise uncorrelated between receivers, $\operatorname{cov}(\mathbf{d})=\operatorname{diag}\left(\sigma^{2}\right)$ is a diagonal matrix, and

$$
\operatorname{cov}(\tilde{\mathbf{m}})=\left(\tilde{\mathbf{F}}^{\mathrm{T}} \tilde{\mathbf{F}}\right)^{-1} \sigma^{2}
$$

For data with unequal uncertainties, the rows of equations (2.1.10) and (2.1.18) are normalized by dividing by the corresponding uncertainties, giving the normalized data unit uncertainties. 


\subsubsection{Principal Moment and Principal Direction Uncertainties}

The leading six by six sub-matrix of $\operatorname{cov}(\tilde{\mathbf{m}})$ gives the covariance of the nonredundant elements of the dipole polarizablity matrix $\mathbf{M}, \operatorname{cov}(\mathbf{m})$. The principal directions of $\mathbf{M}$ are given by the eigenvectors of $\mathbf{M}$, and form the columns of the rotation matrix $\mathbf{U}$ which diagonalizes $\mathbf{M}$ (equation 2.1.7), yielding its the principal moments on the diagonal. Using the symmetry of $\mathbf{M}$ and $\mathbf{L}$, equation (2.1.7) can be written as

$$
\mathbf{l}_{\mathrm{L}}=\mathbf{O} \mathbf{~ m}
$$

where $\mathbf{l}_{\mathrm{L}} \equiv\left(\mathrm{L}_{11}, \mathrm{~L}_{22}, \mathrm{~L}_{33}, \mathrm{~L}_{12}, \mathrm{~L}_{23}, \mathrm{~L}_{13}\right)^{\mathrm{T}}$, and $\mathbf{O}$ is an orthogonal matrix obtained by writing out matrix product (2.1.7) explicitly and identifying coefficients. Principal moments $\mathrm{L}_{11}, \mathrm{~L}_{22}, \mathrm{~L}_{33}$ are Rayleigh quotients of matrix $\mathbf{M}$, so are insensitive to first order to changes in estimated principal direction matrix $\mathbf{U}$. Their squared uncertainties lie on the diagonal of

$$
\operatorname{cov}\left(\mathbf{l}_{\mathrm{L}}\right)=\mathbf{O} \operatorname{cov}(\mathbf{m}) \mathbf{O}^{\mathrm{T}} .
$$

Uncertainties in the principal directions of $\mathbf{M}$ are related to the stability of eigenvectors of $\mathbf{M}$ to changes in $\mathbf{M}$. Perturbing $\mathbf{M}$ by $\Delta \mathbf{M}$, the resulting change in the $\mathrm{j}^{\prime}$ th eigenvector (principal direction) $\mathbf{u}_{\mathrm{j}}$ is

$$
\Delta \mathbf{u}_{\mathrm{j}}=\sum_{\mathrm{k} \neq \mathrm{j}} \frac{\mathbf{u}_{\mathrm{k}}{ }^{\mathrm{T}} \Delta \mathbf{M} \mathbf{u}_{\mathrm{j}}}{\lambda_{\mathrm{j}}-\lambda_{\mathrm{k}}} \mathbf{u}_{\mathrm{k}}
$$

to first order in $\Delta \mathbf{M}$, provided that $\lambda_{\mathrm{j}} \neq \lambda_{\mathrm{k}}$ for $\mathrm{k} \neq \mathrm{j}$, where $\lambda_{\mathrm{k}}$ are eigenvalues of $\mathbf{M}\left(\mathrm{L}_{11}\right.$, $\mathrm{L}_{22}$, and $\mathrm{L}_{33}$ ) (Watson, 1983). The numerator can be written as

$$
\mathbf{u}_{\mathrm{k}}{ }^{\mathrm{T}} \Delta \mathbf{M} \quad \mathbf{u}_{\mathrm{j}}=\mathbf{w}_{\mathrm{jk}}{ }^{\mathrm{T}} \Delta \mathbf{m}
$$

where $\mathbf{w}_{\mathrm{jk}}^{\mathrm{T}} \equiv\left(\mathrm{u}_{1 \mathrm{j}} \mathrm{u}_{1 \mathrm{k}}, \mathrm{u}_{2 \mathrm{j}} \mathrm{u}_{2 \mathrm{k}}, \mathrm{u}_{3 \mathrm{j}} \mathrm{u}_{3 \mathrm{k}}, \mathrm{u}_{1 \mathrm{j}} \mathrm{u}_{2 \mathrm{k}}+\mathrm{u}_{2 \mathrm{j}} \mathrm{u}_{1 \mathrm{k}}, \mathrm{u}_{2 \mathrm{j}} \mathrm{u}_{3 \mathrm{k}}+\mathrm{u}_{3 \mathrm{j}} \mathrm{u}_{2 \mathrm{k}}, \mathrm{u}_{1 \mathrm{j}} \mathrm{u}_{3 \mathrm{k}}+\mathrm{u}_{3 \mathrm{j}} \mathrm{u}_{1 \mathrm{k}}\right)$. The squared uncertainties of the elements of the $j^{\prime}$ th principal direction are then given by the diagonal elements of the three by three matrix

$$
\operatorname{cov}\left(\mathbf{u}_{j}\right)=\left(\sum_{k \neq j} \frac{\mathbf{u}_{k} \mathbf{w}_{j k}^{\mathrm{T}}}{\lambda_{j}-\lambda_{k}}\right) \operatorname{cov}(\mathbf{m})\left(\sum_{k \neq j} \frac{\mathbf{u}_{k} \mathbf{w}_{j k}{ }^{\mathrm{T}}}{\lambda_{j}-\lambda_{k}}\right)^{\mathrm{T}} .
$$

If some eigenvalue $\lambda_{\mathrm{k}}$ is very close to $\lambda_{\mathrm{j}}$ the denominator in equation (2.1.24) becomes small, and a perturbation $\Delta \mathbf{M}$ may perturb the $\mathrm{j}^{\prime}$ th eigenvector a large amount in the direction of the $\mathrm{k}^{\prime}$ th eigenvector. Consequently, principal directions corresponding to 
two principal moments become indeterminate when the difference between the two moments is less than the uncertainty in the difference. The squared uncertainty in the difference between the $i^{\prime}$ th and $j^{\prime}$ th principal moments is

$$
\operatorname{var}\left(\mathrm{L}_{\mathrm{ii}}-\mathrm{L}_{\mathrm{jj}}\right)=\operatorname{cov}\left(\mathbf{l}_{\mathrm{L}}\right)_{\mathrm{ii}}+\operatorname{cov}\left(\mathbf{l}_{\mathrm{L}}\right)_{\mathrm{jj}}-2 \operatorname{cov}\left(\mathbf{l}_{\mathrm{L}}\right)_{\mathrm{ij}},
$$

where $\operatorname{cov}\left(\mathbf{l}_{\mathrm{L}}\right)_{\mathrm{ij}}$ is the $\mathrm{i} \mathrm{j}^{\prime}$ th element of $\operatorname{cov}\left(\mathbf{l}_{\mathrm{L}}\right)$.

\subsubsection{Application}

Our current application of equivalent dipole polarizabilities is discrimination amongst buried metallic objects. The authors' encoding of the preceeding algorithms have been extensively tested on synthetic data. Two synthetic examples are presented here.

The first example simulates collection of magnetic induction data in the vicinity of a $12 \mathrm{~cm}$ diameter buried steel sphere with a relative permeability $\mu_{\mathrm{r}}=180$, and conductivity $\sigma=10^{7} \Omega^{-1} \mathrm{~m}^{-1}$, with the sphere center $1 \mathrm{~m}$ below the level of transmitter and receiver coils. Three components of the time derivative of the secondary magnetic induction $\mathrm{d} \mathbf{B}^{(\mathrm{s})} / \mathrm{dt}$ were computed at the center of a $1 \mathrm{~m}$ square loop transmitter for 81 placements of the loop on a $9 \times 9$ grid with 0.4 m spacing. An observation time of $610 \mu \mathrm{s}$ after transmitter turn-off was chosen to approximate the effective center time of the averaging gate of a commercial transmitter-receiver system (Geonics EM-61). The largest observed derivative component is $\mathrm{dB}_{\mathrm{z}}^{(\mathrm{s})} / \mathrm{dt}$ directly above the sphere. For a 180 Amp- $\mathrm{m}^{2}$ transmitter moment, $\mathrm{dB}_{\mathrm{z}}{ }^{(\mathrm{s})} / \mathrm{dt}=-4648$. $\mathrm{nT} / \mathrm{s}$ for the measurement directly above the sphere at $610 \mu \mathrm{s}$. Gaussian noise of magnitude $8.8 \mathrm{nT} / \mathrm{s}$ was added to the $\mathrm{dB}_{\mathrm{z}}{ }^{(\mathrm{s})} / \mathrm{dt}$ measurements simulating an observed noise level (at Fort Ord, California). Gaussian noise of magnitude 27. $\mathrm{nT} / \mathrm{s}$ was added to the $\mathrm{dB}_{\mathrm{x}}{ }^{(\mathrm{s})} / \mathrm{dt}$ and $\mathrm{dB}_{\mathrm{y}}{ }^{(\mathrm{s})} / \mathrm{dt}$ measurements to simulate the larger noise levels typically observed in horizontal field components. These data were inverted for dipole polarizabilities and location. The downhill simplex algorithm converges to a weighted rms misfit of 0.90318 with the estimated object center at $(\mathrm{x}, \mathrm{y}, \mathrm{z})=(0.0028,-0.0039,1.0008)$ meters. Started from this point, after three iterations the linearized inversion converges to a weight rms misfit of 0.90316 with the estimated object center at $(0.0026 \pm 0.0030,-0.0040 \pm 0.0030,1.0002 \pm 0.0051)$ meters. The true center position is $(0,0,1)$ meters. The estimated principal dipole polarizabilities 
$\mathrm{L}_{11}, \mathrm{~L}_{22}$, and $\mathrm{L}_{33}$ are $-0.654 \pm 0.015,-0.647 \pm 0.011,-0.635 \pm 0.014 \mathrm{Amp}-\mathrm{m}^{2} / \mathrm{s} / \mu \mathrm{T}$. The absolute differences between principal dipole polarizability estimates $0.008 \pm 0.010$ and $0.012 \pm 0.009$ Amp-m $\mathrm{m}^{2} / \mathrm{s} / \mu \mathrm{T}$ respectively for $\left|\mathrm{L}_{11}-\mathrm{L}_{22}\right|$ and $\left|\mathrm{L}_{22}-\mathrm{L}_{33}\right|$, are less than two estimation errors, indicating that the object is spherically symmetric within measurement errors.

For a second example, the response of an aluminum prolate spheroid $24 \mathrm{~cm}$ long by $8 \mathrm{~cm}$ wide, of conductivity $\sigma=3.510^{7} \Omega^{-1} \mathrm{~m}^{-1}$ was modeled using an integral equation code provided by P. B. Weichman of Blackhawk Geophysics, with subsequent modifications to improve accuracy. The code expands the electric field within the spheroid in a polynomial basis, and solves for a set of modes, each with a characteristic decay time. Subsequently, the excitation of the modes for each position of transmitter loop is computed for a ramp-on/ramp-off transmitter current, and the contributions of the different mode voltages observed in receiver coils are summed over modes, for each transmitter-receiver pair. This code was used to compute the spheroid response for 81 transmitter loop positions of a $1 \mathrm{~m}$ square horizontal loop, on a 9 by 9 grid with $0.2 \mathrm{~m}$ spacing, in two coaxial dipole receivers, one concentric with the transmitter, and the other $0.4 \mathrm{~m}$ above the first. The spheroid center was placed $0.6 \mathrm{~m}$ below the transmitter level, offset $0.2 \mathrm{~m}$ in $\mathrm{x}$ and $\mathrm{y}$ from the grid center, with symmetry axis in the $\mathrm{y}$-z plane dipping $30^{\circ}$. A $3.3 \mathrm{~ms}$ ramp-on, $0.08 \mathrm{~ms}$ ramp-off transmitter current, and a $0.4 \mathrm{~ms}$ averaging gate starting $0.42 \mathrm{~ms}$ after transmitter current extinction, were used to emulate a commercial transmitter-receiver system (Geonics EM-61).

Gaussian noise with a magnitude of $1 \%$ of the largest observed voltage was added to the computed voltages. The resultant data was inverted, yielding an estimated center location of $(0.207 \pm 0.008,0.206 \pm 0.009,0.600 \pm 0.005)$ meters, in agreement with the true location $(0.200,0.200,0.600)$. The principal polarizabilities were estimated as 0.785 $\pm 0.023,0.768 \pm 0.018$, and $0.529 \pm 0.011 \mathrm{~V} / \mu \mathrm{T}$, with differences $0.016 \pm 0.025$ and $0.238 \pm 0.020 \mathrm{~V} / \mu \mathrm{T}$. The agreement of $\mathrm{L}_{11}$ and $\mathrm{L}_{22}$ indicates an object that, within measurement errors, is rotationally symmetric about the third principal direction. The differences between the third moment and the other two are well resolved, and, for a nonmagnetic object, consistent with the smaller cross section perpendicular to the symmetry 
axis. The third principal direction is estimated as $(0.016 \pm 0.023,0.850 \pm 0.026,-0.526 \pm$ $0.043)$, in agreement with the true axis of symmetry $(0,0.866,-0.500)$.

For the transmitter-receiver configurations used in the above examples (which each have more than one receiver component of data), examination of plots of the squared data misfit $\chi^{2}$ as a function of candidate center position (not shown), on sections through, and near, the true object location, typically show a large "valley" in data misfit sloping downwards to a well defined minimum at the object center (within measurement errors), with small shallow secondary valleys close to the transmitters and receivers. Close to the transmitters and receivers, misfit topography is on the scale of the data grid spacing. The existence of such topography recommends measurement grid spacings finer than the height of the transmitters and receivers above the shallowest expected depth of objects (e.g., ground surface).

For the commonly occurring case of a transmitter-receiver system with a single horizontal transmitter loop and single coaxial receiver, plots of squared data misfit as a function of candidate center position are more problematic. Figure 2.1.1 shows a detail of such a plot, for a $1 \mathrm{~m}^{2}$ horizontal loop transmitter, concentric $B_{z}$ receiver system, sited on a 9 by 9 grid centered over a sphere at $(0,0,1)$ meters. There is a clear minimum at the true object center $(0,0,1)$, but also a shallow local minimum at $(0.00,0.00,1.08)$ meters. The existence of local minima close to, but distinct from, the minimum associated with the true object position, means that one must be extremely cautious in interpreting data from single transmitter single receiver systems. For comparison, Figure 2.1.2 shows squared data misfit $\chi^{2}$ over the same region of candidate object center position, for a similar system with an additional $\mathrm{B}_{\mathrm{z}}$ receiver $0.4 \mathrm{~m}$ above the first. The added data eliminates the secondary local minimum. 


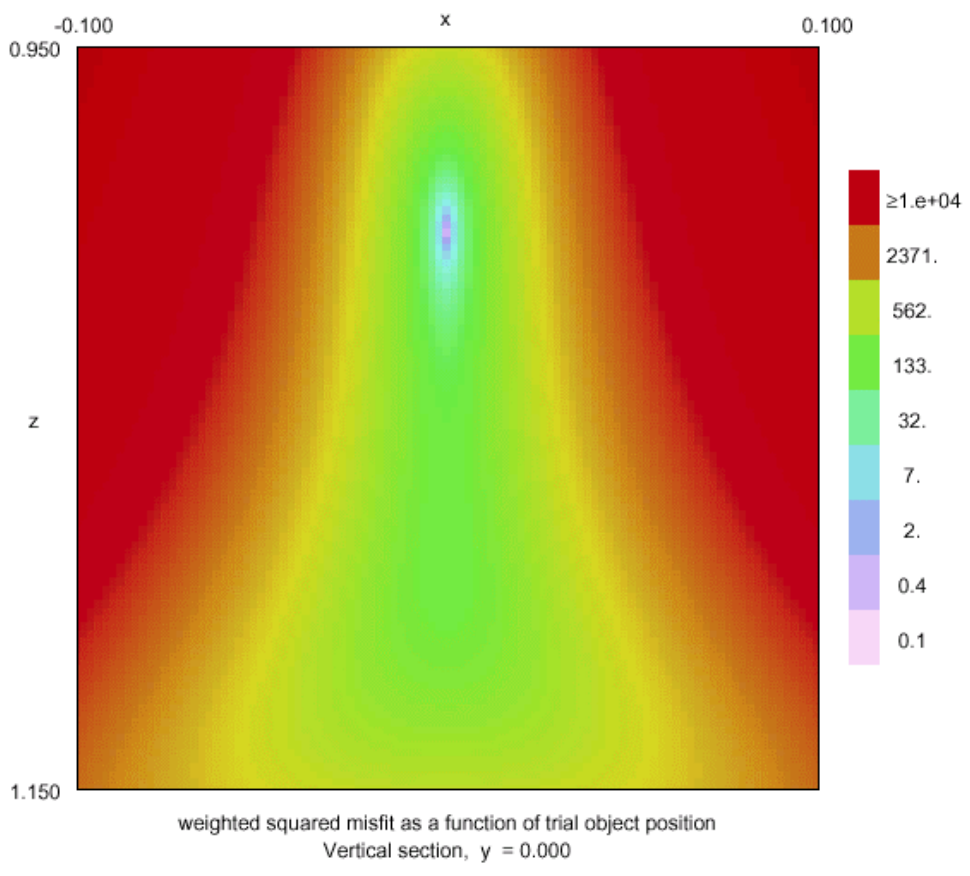

Figure 2.1.1: Squared data misfit $\chi^{2}$ as a function of candidate object center position $r_{0}$, for $1 \mathrm{~m}^{2}$ square transmitter loop, concentric vertical dipole receiver system sited on a $9 \mathrm{x}$ 9 grid, centered $1 \mathrm{~m}$ above a $12 \mathrm{~cm}$ steel sphere (detail).

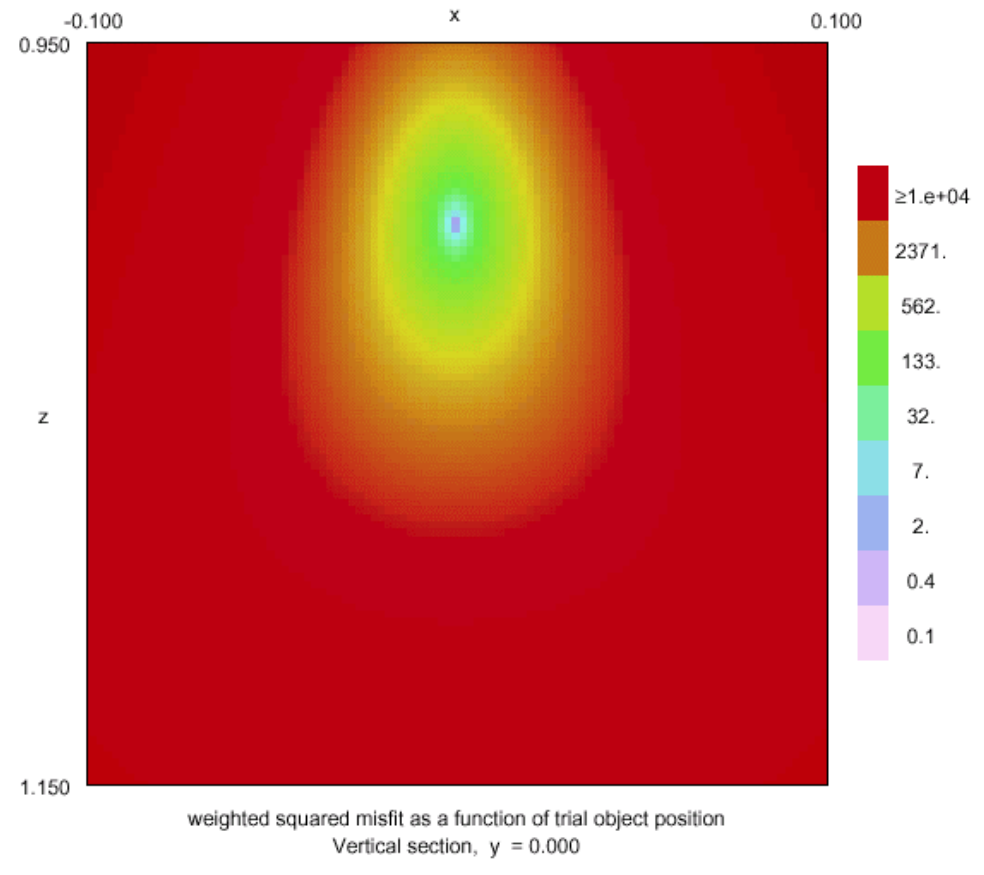

Figure 2.1.2: Squared data misfit $\chi^{2}$ as a function of candidate object center position $r_{0}$, for $1 \mathrm{~m}^{2}$ square transmitter loop, 2 coaxial vertical dipole receiver system sited on a $9 \times 9$ grid, centered $1 \mathrm{~m}$ above a $12 \mathrm{~cm}$ steel sphere (detail). 


\subsection{Depths of equivalent dipole polarizability resolution for some transmitter receiver configurations}

Equivalent dipole polarizability matrices and dipole locations are a convenient way to summarize active source induced current magnetic field measurements in an interpretable form. The matrices' principal moments give information on the rotational symmetry of a conductive object, their principal directions yield the object's orientation, and the dipole location $\mathbf{r}_{0}$ estimates the object's center position. An algorithm for estimating these and their uncertainties was outlined in a previous section. Here uncertainty estimates are used to compute the depths to which the polarizability matrices and dipole locations can be estimated for steel spheres of varying radius, for several transmitter-receiver configurations.

Equivalent dipole polarizability matrices $\mathbf{M}$ model observed secondary magnetic fields $\mathbf{B}^{(\mathrm{s})}(\mathbf{r}, \mathrm{t})$, in terms of the magnetic fields of unit dipoles in the $\hat{\mathbf{x}}, \hat{\mathbf{y}}$, and $\hat{\mathbf{z}}$ directions, $\mathbf{B}_{\mathrm{x}}{ }^{(\mathrm{d})}(\mathbf{r}), \mathbf{B}_{\mathrm{y}}{ }^{(\mathrm{d})}(\mathbf{r}), \mathbf{B}_{\mathrm{z}}{ }^{(\mathrm{d})}(\mathbf{r})$ centered at some location $\mathbf{r}_{0}$, and the primary (inducing) magnetic field strength $\mathbf{B}^{(0)} \cdot g(t)$, at $\mathbf{r}_{0}$, for a given time variation $g(t)$ of primary magnetic field:

$$
\mathbf{B}^{(\mathrm{s})}(\mathbf{r}, \mathrm{t})=\left[\mathbf{B}_{\mathrm{x}}{ }^{(\mathrm{d})}(\mathbf{r}), \mathbf{B}_{\mathrm{y}}{ }^{(\mathrm{d})}(\mathbf{r}), \mathbf{B}_{\mathrm{z}}{ }^{(\mathrm{d})}(\mathbf{r})\right] \mathbf{M}(\mathrm{t}) \mathbf{B}^{(0)}
$$

In this model, the polarizability matrix is independent of transmitter and receiver geometry and object location, depending only on the innate properties of the object, its orientation, and the transmitter waveform. The principal values of $\mathbf{M}$, depend only on the object, independent of its orientation, and on the transmitter waveform. Equivalent dipole position $\mathbf{r}_{0}$ is generally assumed to coincide with the object center.

For typical time domain systems, secondary fields are measured after primary fields are extinct, at which time the entire magnetic field is secondary. In simplest form, the time variation of the primary field is incorporated in the estimated polarizability matrix $\mathbf{M}(\mathrm{t})$, and the primary magnetic field strength vector at object center $\mathbf{B}^{(0)}$, for a given source, is simply the magnetic field there for a D.C. current in the transmitter coils of the transmitter's nominal current strength (e.g., peak current strength). 
The methods of Section 2.1 were used to estimate equivalent dipole polarizabilities from synthetic three component magnetic field data for a vertical magnetic dipole source at 13 sites placed symmetrically on a grid with $0.4 \mathrm{~m}$ spacings in $\mathrm{x}$ and $\mathrm{y}$ centered 1 meter above steel spheres of varying radius, modeled with a conductivity of $\sigma=10^{7} \Omega^{-1} \mathrm{~m}^{-1}$ and relative permeability $\mu_{\mathrm{r}}=180$. A step function turn-off transmitter current was used, as the most generic of waveforms, and an observation time of $610 \mu \mathrm{s}$ after turnoff chosen to simulate the effective center time of the averaging gate of an existent commercial transmitter-receiver system. Polarizability estimates are listed in Table I. In principle, for spherically symmetric objects, the three principal moments are identical. The variation between estimated principal moments is less than $1 \%$ for spheres smaller than $25 \mathrm{~cm}$ radius. The variations are due to the limited spatial extent of the data used, the presence of non-dipole moment components in the data, and truncation of the data at four significant figures. In subsequent computations based on Table I, the three estimated moments were replaced with their average.

\begin{tabular}{c|ccc} 
Radius $(\mathrm{m})$ & \multicolumn{4}{|c}{ Estimated Principal Moments $\left(\mathrm{Amp}-\mathrm{m}^{2} / \mathrm{s} / \mathrm{T}\right)$} \\
\hline 0.01 & $-7.28310^{3}$ & $-7.27510^{3}$ & $-7.27510^{3}$ \\
0.02 & $-1.11410^{4}$ & $-1.11410^{4}$ & $-1.11310^{4}$ \\
0.03 & $-5.26210^{4}$ & $-5.26210^{4}$ & $-5.26210^{4}$ \\
0.04 & $-1.53010^{5}$ & $-1.53010^{5}$ & $-1.52910^{5}$ \\
0.05 & $-3.40510^{5}$ & $-3.40510^{5}$ & $-3.39910^{5}$ \\
0.06 & $-6.41610^{5}$ & $-6.41610^{5}$ & $-6.40110^{5}$ \\
0.08 & $-1.67210^{6}$ & $-1.67210^{6}$ & $-1.66410^{6}$ \\
0.10 & $-3.38210^{6}$ & $-3.38210^{6}$ & $-3.35510^{6}$ \\
0.15 & $-1.12110^{7}$ & $-1.12110^{7}$ & $-1.11210^{7}$ \\
0.25 & $-4.41810^{7}$ & $-4.41810^{7}$ & $-4.37310^{7}$ \\
0.50 & $-2.35310^{8}$ & $-2.35310^{8}$ & $-2.28510^{8}$ \\
1.00 & $-1.09810^{9}$ & $-1.09810^{9}$ & $-1.05710^{9}$
\end{tabular}

Table I. Estimated principal moments for simulated steel sphere data at $610 \mu \mathrm{s}$ after step function turn-off, from 13 vector measurements coincident with vertical dipole source on grid $1 \mathrm{~m}$ above sphere center. 
Section 2.1 gives equations for the covariance matrix for dipole polarizability matrix $\mathbf{M}$ elements, and covariance matrices for the principal moments and directions derived from it. In general, computing principal moments and directions requires knowing all elements of $\mathbf{M}$ (which is symmetric). If principal directions are known a priori, principal moments may be determined from fewer measurements, but determining the principal directions empirically requires knowledge of all elements of $\mathbf{M}$. A simple measure of how well a data set resolves $\mathbf{M}$ is the relative average squared uncertainty

$$
\xi^{2} \equiv \sum_{\mathrm{i}=1}^{3} \sum_{\mathrm{j}=1}^{3} \operatorname{var}\left(\mathrm{m}_{\mathrm{ij}}\right) / \sum_{\mathrm{i}=1}^{3} \sum_{\mathrm{j}=1}^{3} \mathrm{~m}_{\mathrm{ij}}^{2}
$$

where $\operatorname{var}\left(\mathrm{m}_{\mathrm{ij}}\right)$ is the estimated squared uncertainty of the $\mathrm{ij}$ 'th element of $\mathbf{M}$ obtained from the diagonal of the covariance matrix of the elements of $\mathbf{M}(\operatorname{cov}(\mathbf{m})$ of Section 2.1). The denominator in (2.2.2) is a matrix invariant; independent of the coordinates used to express $\mathbf{M}$, so a property of the object from which $\mathbf{M}$ arises. In our experience, the numerator of (2.2.2) is also coordinate independent, suggesting that $\xi^{2}$ itself may be a coordinate independent measure of the relative uncertainty in $\mathbf{M}$.

For each radius sphere in Table I, for each of a number of transmitter-receiver configurations, the relative root mean squared (rms) moment uncertainty $\xi$ was computed as a function of sphere depth, for spheres directly below the center of a $9 \times 9$ grid of system placements with $0.4 \mathrm{~m}$ spacing in $\mathrm{x}$ and $\mathrm{y}$. One meter square transmitter loops were used with a moment of $180 \mathrm{Amp}-\mathrm{m}^{2}$, and a receiver noise level of $1.97 \mathrm{nT} / \mathrm{s}$ in vertical field measurements, simulating an observed noise level, and $5.91 \mathrm{nT} / \mathrm{s}$ in horizontal field components (when present) simulating the larger noise levels observed in horizontal components.

A plot of relative rms moment uncertainty $\xi$ as a function of depth below transmitter and receiver is shown in Figure 2.2.1 for an isotropic $-6.4110^{5} \mathrm{Amp}-\mathrm{m}^{2} / \mathrm{s} / \mathrm{T}$ equivalent dipole polarizability $(6 \mathrm{~cm}$ radius steel sphere at $610 \mu \mathrm{s})$ below the grid of system placements for a horizontal loop transmitter / concentric vertical magnetic dipole receiver system. Being based on a linearized inversion for $\mathbf{M}$ and $\mathbf{r}_{0}$, these uncertainty estimates scale linearly with receiver noise level. For spheres very near the level of the transmitter and receiver $(\mathrm{z}=0)$, the rms uncertainty is large as all the transmitter placements illuminate the sphere with nearly vertical primary fields, yielding little information on $\mathrm{m}_{\mathrm{xx}}$, 
$\mathrm{m}_{\mathrm{xy}}$, and $\mathrm{m}_{\mathrm{yy}}$, and correspondingly large variances in them. Relative rms uncertainty decreases to a minimum at $0.135 \mathrm{~m}$ depth, and subsequently rises with increasing depth, reflecting the decrease of primary field strengths with increasing depth. In practice, mounting a receiver-transmitter system above ground level sets a minimum depth below transmitter for buried objects, avoiding difficulties with the large uncertainties at the transmitter-receiver level. The great increase of uncertainty with depth for spheres at large depths limits the depths for which polarizabilities can be resolved. For the horizontal loop transmitter vertical dipole receiver on this grid $\xi>0.1$ for $6 \mathrm{~cm}$ radius steel spheres below $1.47 \mathrm{~m}$. (At $\mathrm{z}=1.47 \mathrm{~m}$, the corresponding relative uncertainties in horizontal and vertical polarizabilities are $0.061,0.061$, and 0.147 respectively.)

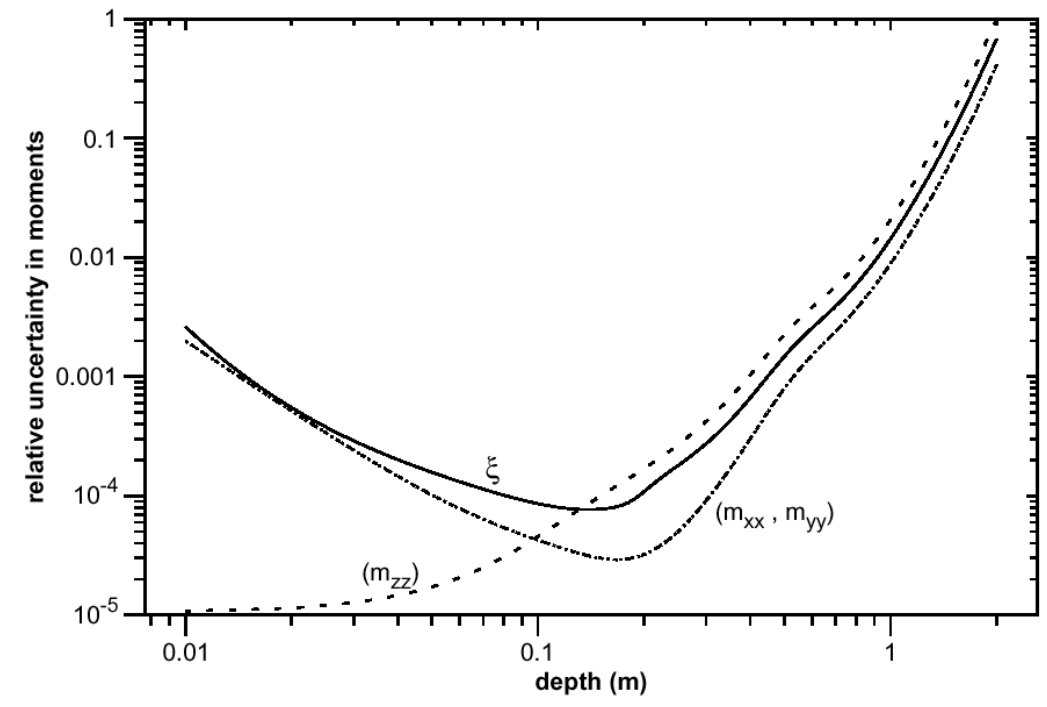

Figure 2.2.1: Relative rms polarizability uncertainty $\xi$ as a function of sphere center depth below transmitter and receiver for a $6 \mathrm{~cm}$ radius steel sphere. Also, relative uncertainty in vertical moment $\mathrm{dm}_{\mathrm{zz}} / \mathrm{dt}$ (dashed), and in horizontal moments $\mathrm{dm}_{\mathrm{xx}} / \mathrm{dt}$ and $\mathrm{dm}_{\mathrm{yy}} / \mathrm{dt}$ (dotted).

The depths to 5,10 , and $20 \%$ rms uncertainty in polarizability $\mathrm{d}_{5 \%}{ }^{(\mathrm{p})}, \mathrm{d}_{10 \%}{ }^{(\mathrm{p})}$, and $\mathrm{d}_{20 \%}{ }^{(\mathrm{p})}$, were similarly found for isotropic polarizabilities corresponding to all the spheres in Table I, and are plotted in Figure 2.2.2, as a function of sphere radius. The depths to 5, 10, and $20 \%$ uncertainty in estimated sphere depth, $\mathrm{d}_{5 \%}{ }^{(\mathrm{z})}, \mathrm{d}_{10 \%}{ }^{(\mathrm{z})}$, and $\mathrm{d}_{20 \%}{ }^{(\mathrm{z})}$, are plotted in Figure 2.2.3. In general, object position can be estimated more precisely than the full polarizability matrix can, as object position may be determined when an object is illuminated by only a single orientation of primary field, whereas estimating the full 
polarizability matrix requires illuminating the object with primary fields $\mathbf{B}_{0}$ in at least three directions, each with a significant component in the direction orthogonal to the other two. Consequently, object depth can be resolved within $10 \%$ to greater depths than polarizability in all cases plotted. The relative uncertainties in polarizability and position depend on all elements of the polarizability matrix $\mathbf{M}$ (through $\tilde{\mathbf{F}}, \mathrm{g}_{\mathrm{i}}{ }^{(\mathrm{q})}$, and $\mathrm{m}_{\mathrm{kj}}{ }^{(\mathrm{q})}$ of Section 2.1), depending on both its principal values, and principal directions (object orientation). Most cases presented here are for spherical objects, for which the principal values are all the same, and the polarizability $\mathbf{M}$ is coordinate independent. For comparison $\mathrm{d}_{10 \%}{ }^{(p)}$ and $\mathrm{d}_{10 \%}{ }^{(\mathrm{z})}$ are plotted in Figure 2.2.4 for the same transmitter-receiver pair, for the case of objects with the same vertical dipole polarizabilities $\mathrm{d} \mathrm{m}_{\mathrm{zz}} / \mathrm{dt}$ at $610 \mu \mathrm{s}$ as the spheres of Table I, and all other polarizabilities null, corresponding to thin horizontal non-magnetic discs. The general trends are the same as for the sphere. Polarizability can be resolved to $10 \%$ slightly deeper than for the sphere for all but the $1 \mathrm{~cm}$ radius sphere. Object depth can be resolved approximately 1.2 times deeper than for the sphere.

Depth to $5 \%, 10 \%, 20 \%$ moment uncertainty

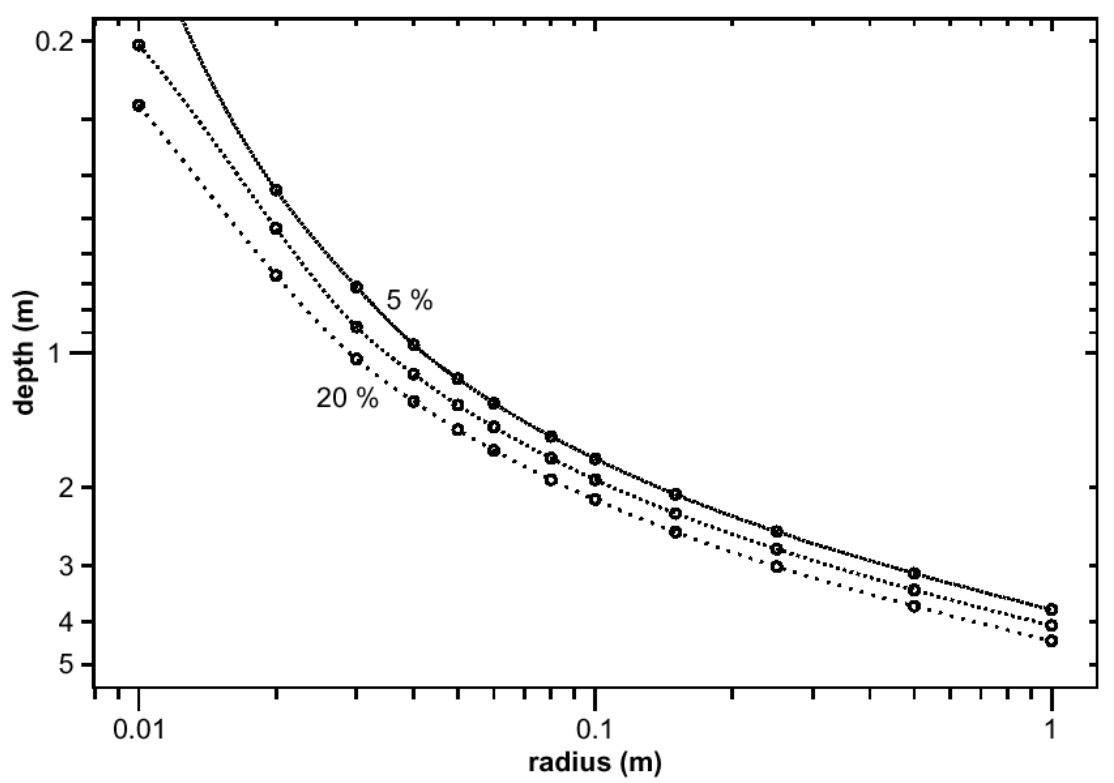

Figure 2.2.2: Depths to $5 \%, 10 \%$, and $20 \%$ rms uncertainty in polarizability as a function of sphere radius for steel spheres below a 9 x 9 grid of transmitter-receiver system placements, for a $1 \mathrm{~m}^{2}$ square loop transmitter with a concentric vertical dipole receiver. 
Depth to $5 \%, 10 \%, 20 \%$ uncertainty in $z$

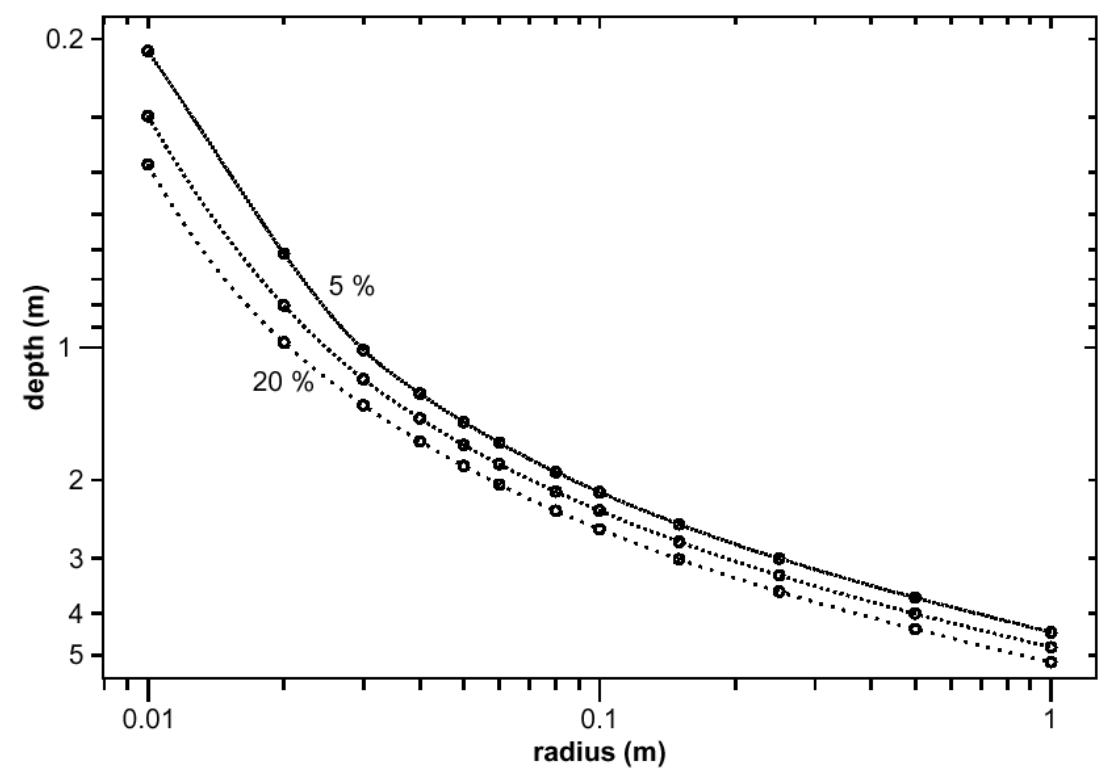

Figure 2.2.3: Depths to $5 \%, 10 \%$, and $20 \%$ uncertainty in sphere center depth, as a function of sphere center depth below center of transmitter-receiver placement grid, for same system and grid as in Figure 2.2.2.

Depth to $10 \%$ uncertainty

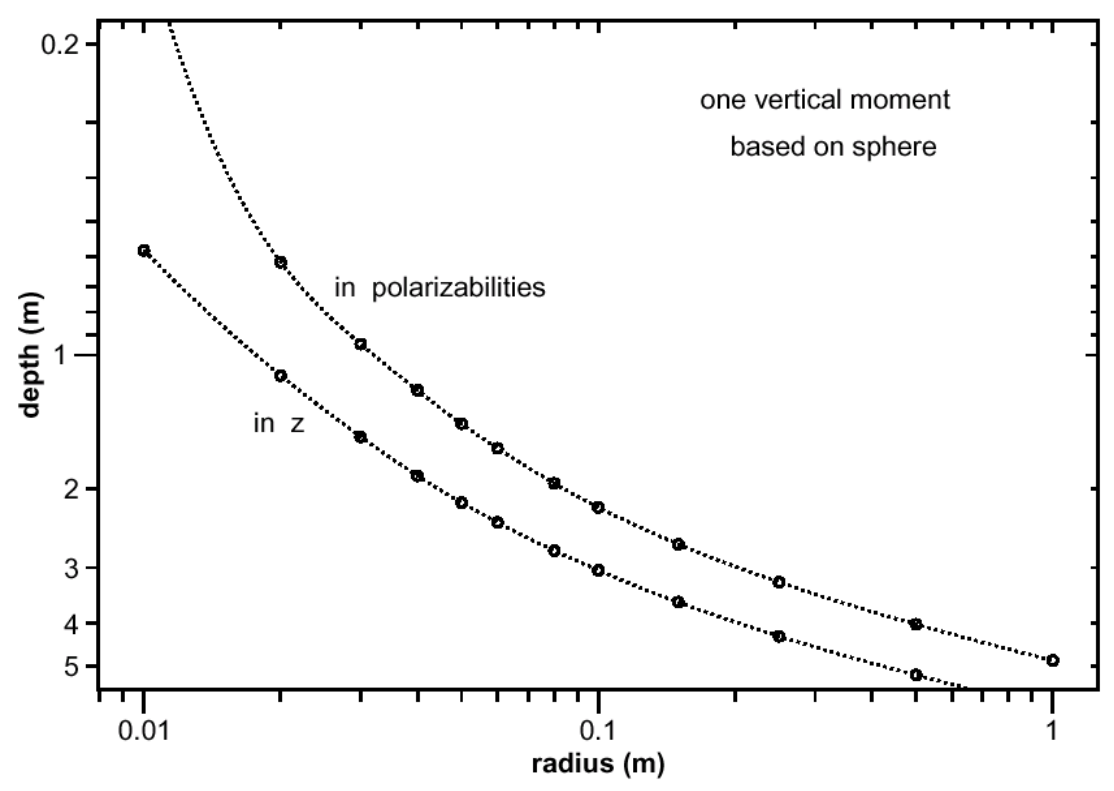

Figure 2.2.4: Depths to $10 \%$ rms polarizability uncertainty and to $10 \%$ uncertainty in center depth, for thin horizontal non-magnetic disk with same vertical polarizability $\mathrm{dm}_{\mathrm{zz}} / \mathrm{dt}$ at $610 \mu \mathrm{s}$, as spheres of Table I, plotted as a function of the corresponding sphere's radius. 
Effects of adding a second coaxial vertical receiver $0.2 \mathrm{~m}$ above the first are shown in Figure 2.2.5, where $\mathrm{d}_{10 \%}{ }^{(\mathrm{p})}$ and $\mathrm{d}_{10 \%}{ }^{(\mathrm{z})}$ are plotted for the two receiver system (dotted) together with their values for the previously plotted single receiver system (solid). In addition to resolving polarizabilities and location to greater depth as shown here, the added receiver makes locating the object position an easier problem as it eliminates a secondary minimum in data misfit near the true object position (Section 2.1).

\section{Depth to $10 \%$ uncertainty}

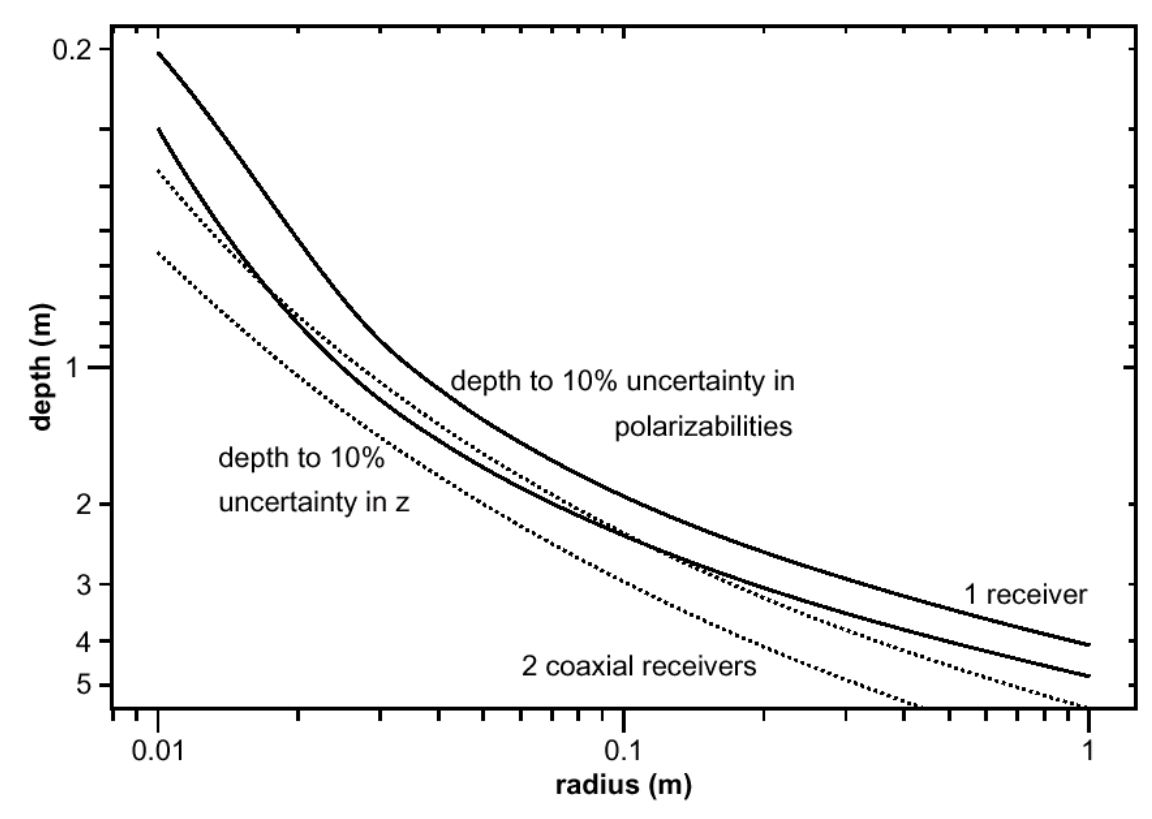

Figure 2.2.5: Depths to $10 \%$ rms polarizability uncertainty and to $10 \%$ uncertainty in center depth, as a function of radius for steel spheres below a $1 \mathrm{~m}^{2}$ square transmitter loop with two coaxial vertical component receivers $0.2 \mathrm{~m}$ apart vertically, on same grid as in Figure 2.2.2.

Figure 2.2.6 plots where $\mathrm{d}_{10 \%}{ }^{(\mathrm{p})}$ and $\mathrm{d}_{10 \%}{ }^{(\mathrm{z})}$ (both dotted) for a similar system with the two vertical component receivers in the plane of the transmitter loop, offset $\pm 0.2 \mathrm{~m}$ in $\mathrm{x}$ and $y$ along a diagonal from the loop center. This system shows greatest improvement in sensitivity over the single receiver system for objects close to the transmitter-receiver plane, greatly increasing the depths to which the smallest spheres can be resolved. For spheres of radii greater than $8 \mathrm{~cm}$, the (modest) improvement is due primarily to a simple 
reduction in uncertainty by a factor of $1 / \sqrt{2}$ due to the doubling of the number of receivers.

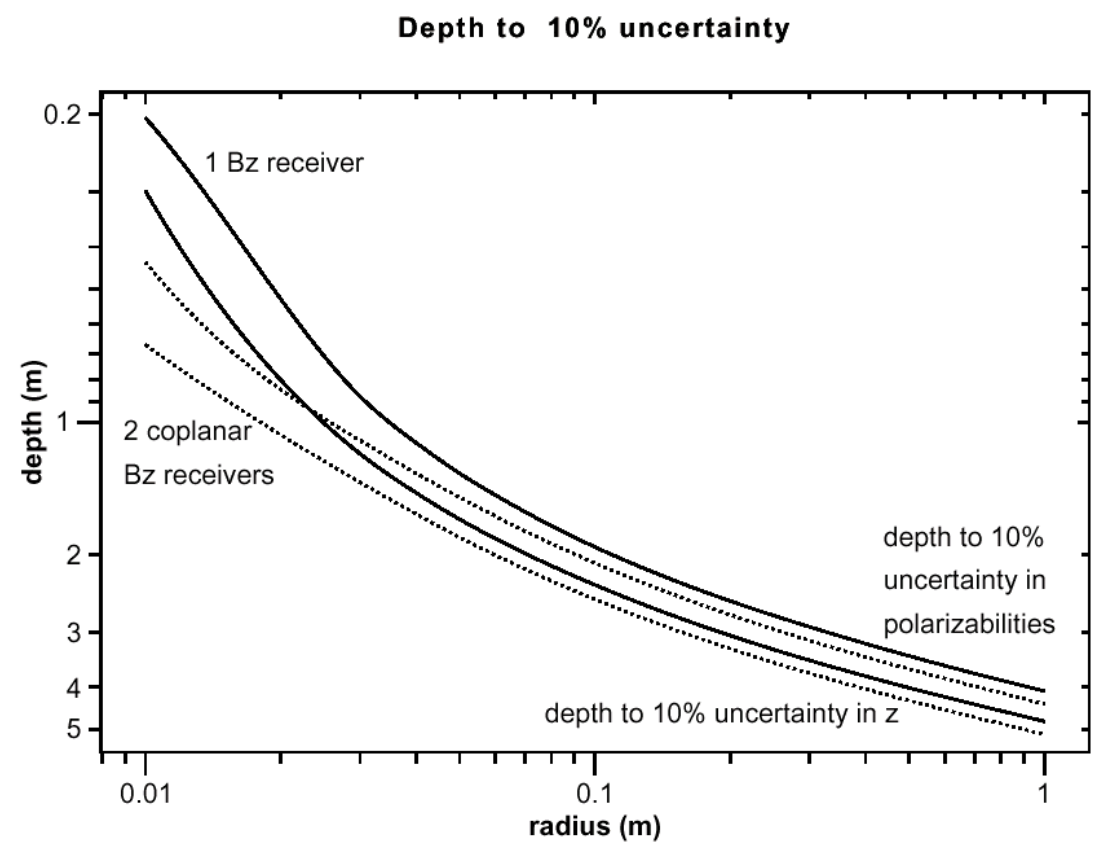

Figure 2.2.6: Depths to $10 \%$ polarizability uncertainty and to $10 \%$ uncertainty in center depth, as a function of sphere radius, for $1 \mathrm{~m}^{2}$ loop transmitter system with two vertical component receivers $0.566 \mathrm{~m}$ apart on diagonal in plane of transmitter loop, on same grid as in Figure 2.2.2.

Results for a horizontal loop transmitter three component concentric receiver are shown in Figure 2.2.7 (dotted lines). For comparison, a system with a $1 \mathrm{~m}^{2}$ horizontal loop and two orthogonal $1 \mathrm{~m}^{2}$ vertical loop transmitters with lower edges at the level of the horizontal loop is shown in Figure 2.2.8 (dotted lines). The results for the 3 transmitter 1 receiver system are substantially better than for the 1 transmitter 3 receiver system, in part, because of the greater noise level in horizontal component receivers. 


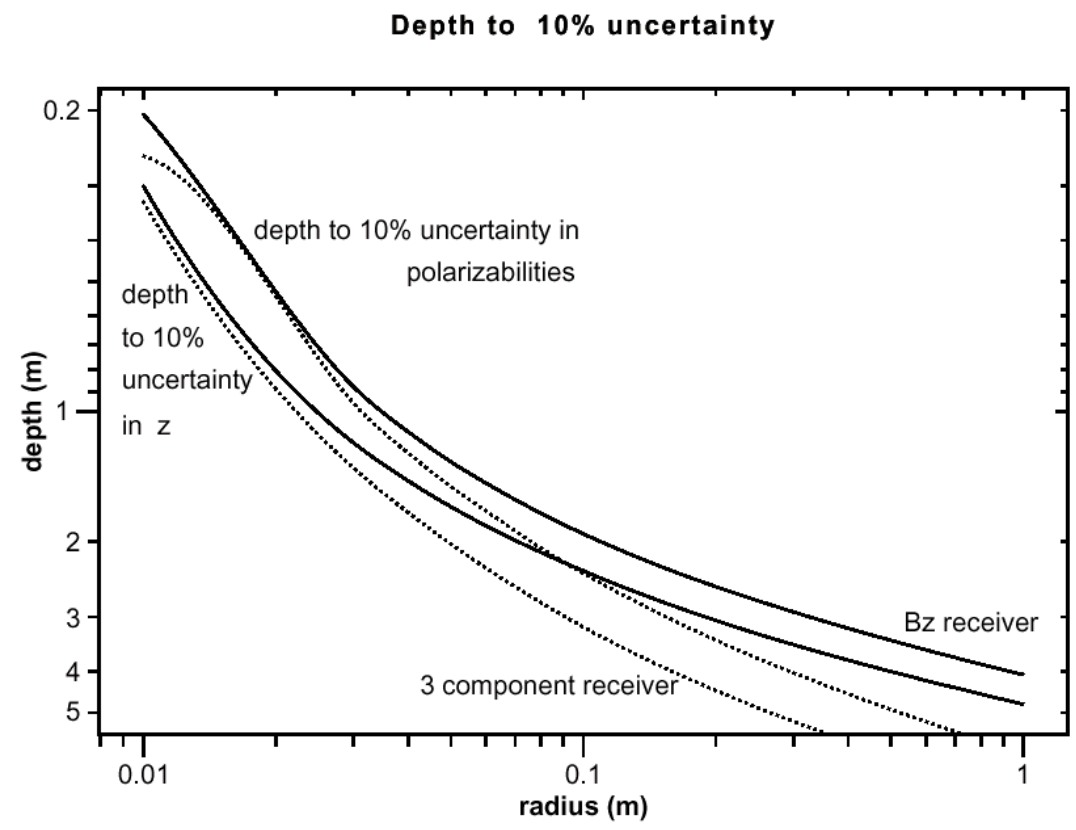

Figure 2.2.7: Depths to $10 \%$ polarizability uncertainty and to $10 \%$ uncertainty in depth as a function of sphere radius, for $1 \mathrm{~m}^{2}$ loop transmitter system with 3 component concentric receiver, on same grid as in Figure 2.2.2.

Depth to $10 \%$ uncertainty

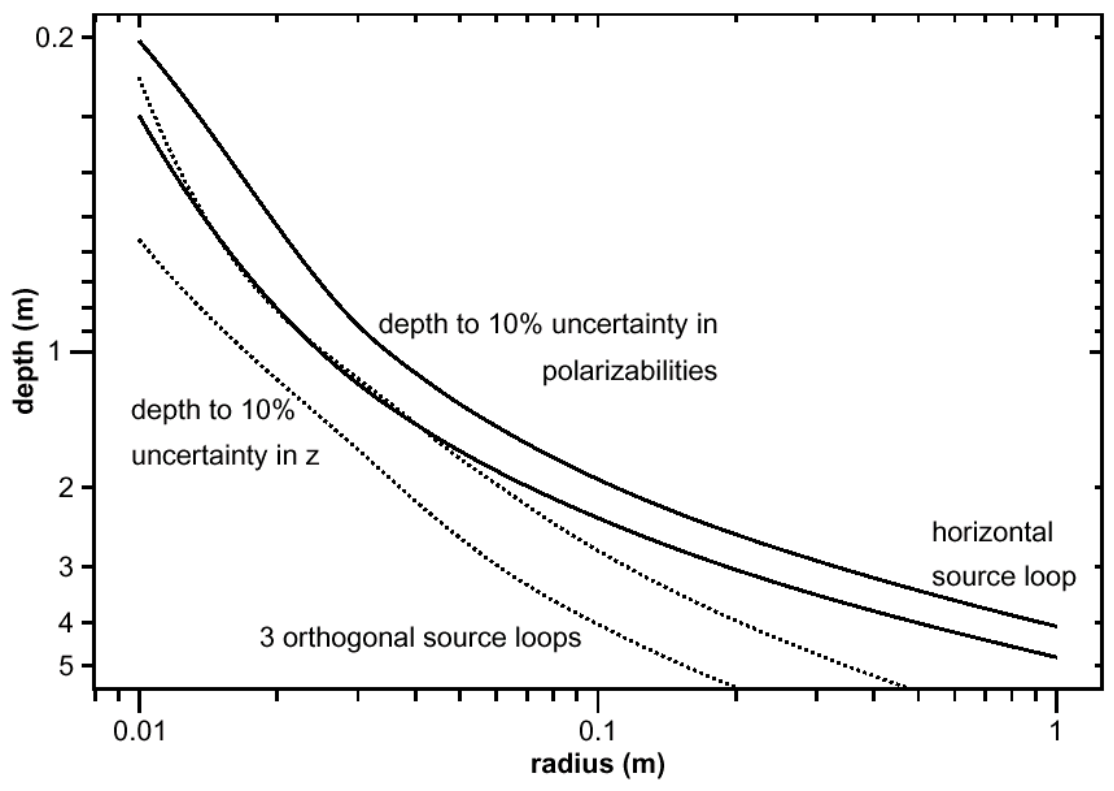

Figure 2.2.8: Depths to $10 \%$ polarizability uncertainty and to $10 \%$ uncertainty in depth, as a function of sphere radius, for three orthogonal $1 \mathrm{~m}^{2}$ loop transmitter system with vertical component receiver at horizontal loop center, on same grid as in Figure 2.2.2. 
As a final example, adding 2 orthogonal horizontal component receivers to the 3 transmitter system to make a 3 transmitter 3 receiver system yields results shown in Figure 2.2.9. The added horizontal components substantially increase the depth of resolution of the $1 \mathrm{~cm}$ radius sphere, but little affect results for $3 \mathrm{~cm}$ radius and larger spheres.

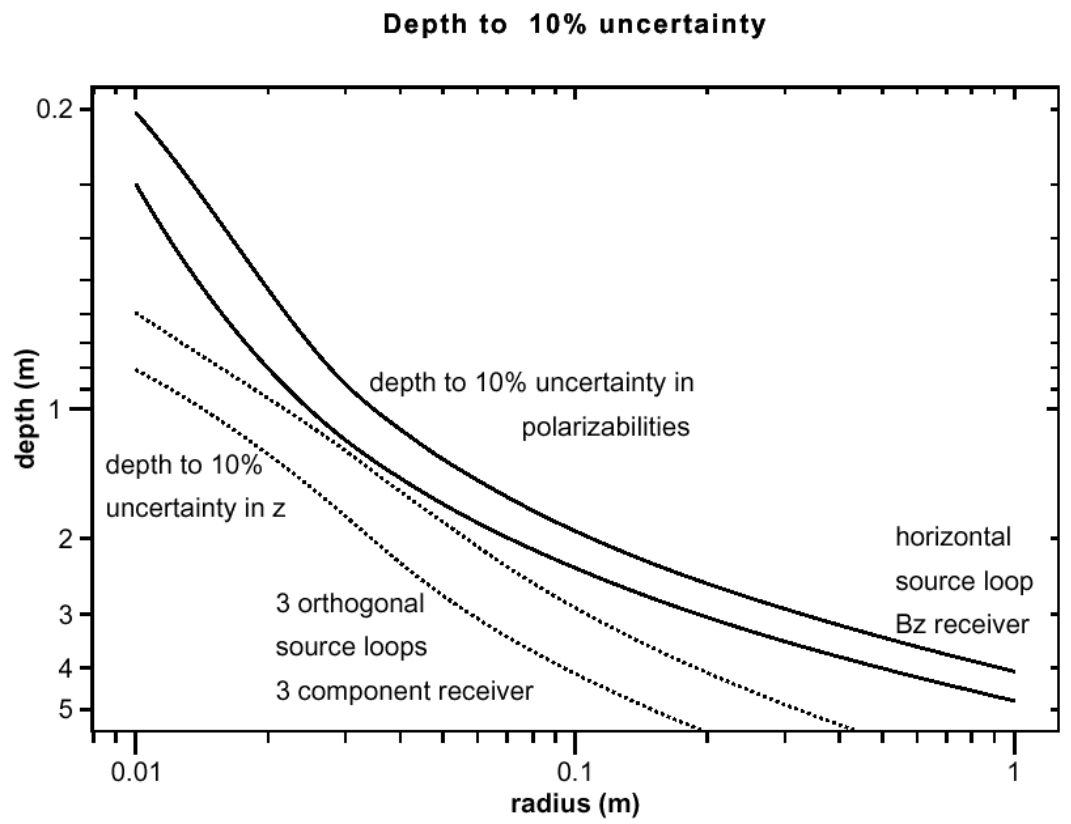

Figure 2.2.9: Depths to $10 \%$ polarizability uncertainty and to $10 \%$ uncertainty in depth, as a function of sphere radius, for 3 transmitter loop system with 3 component receiver at horizontal loop center, on same grid as in Figure 2.2.2. 


\section{PERFORMANCE ASSESSMENT AND DIRECT OPTIMIZATION}

\subsection{Introduction}

The broad definition of optimum in this report is an active EM system that can extract, from the measurements, the best possible estimates of the location, orientation, depth, size, shape, and metal content of a buried metallic object in the presence of the interfering response of non-UXO metallic objects. Discrimination can be achieved partly through selective filtering of the response inherent in the system design and partly through post acquisition processing. These parameters are never determined perfectly because of a fundamental non-uniqueness in the solutions (the response of the target) to the governing equations. Also, practical limitations introduced by depth of the target, the response of a general, inhomogeneous, ground and the presence of response from other non-UXO metallic objects, as well as the signal to noise limitations imposed by weight and power considerations, introduce constraints on what is achievable. An optimum system is bounded by these theoretical and practical considerations and at the end represents a compromise that detects, discriminates and classifies to an agreed upon criterion or specification.

There have been several theoretical and numerical studies of target response for new UXO EM systems, none of which have addressed the issue of optimization. Laboratory, field and theoretical analyses of the frequency domain GEM-3 (e.g. Won et al., 1997) and of the EM-61 and its variants EM61HH, EM63, etc. (e.g. McNeill and Bosnar, 1996; Khadr et al., 1998; Ware, 2000) for a range of targets have demonstrated some of the detection/classification properties and potential of these systems. The studies focused on the response of a particular system to various targets but not on the broader issue of what system would perform best over the targets. Some of these systems rely on the frequency domain (spectral) or time domain transient signal characteristics to classify the target (e.g. Keiswetter et al., 1999; Barrow et al. 1996; or Ware, 2000). Others rely on both the spectral response and the variation of response with transmitted field polarization (e.g. Bell et al., 1998; Khadr et al., 1998; Pasion and Oldenburg, 2001; Snyder et al., 1999). A fundamental and critical technical comparison of time and frequency approaches has been tactfully avoided. 
Our research has clearly shown the importance of using multiple polarizations of the incident magnetic field to stimulate the principal induced magnetic polarizations of the target. The second step in the identification/discrimination process is then to measure the resulting secondary fields at enough points in space to uniquely determine these principal polarizations - the inversion or interpretation process.

[Fundamentally the incident magnetic field induces a circulating current in a confined conductor. This current produces a magnetic dipole moment, usually referred to as M. If this moment is normalized by the inducing magnetic field, the resulting quantity is called the magnetic polarization. We use both terms in this report.] Finally the characterization depends on recovering the broadband polarization response.

The concept of identifying an object through the measurement of secondary fields arising from induced magnetization and induced currents is illustrated in the following cartoon.
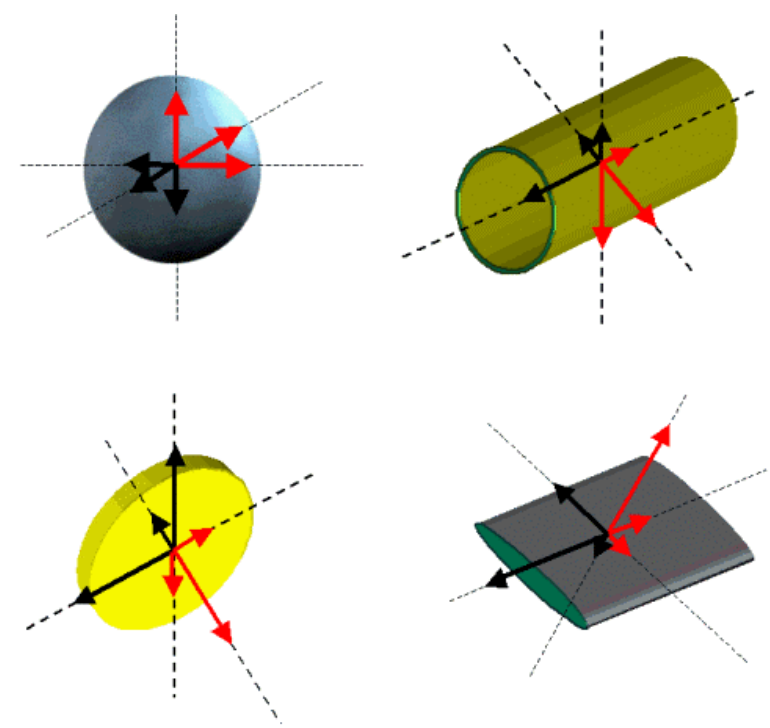

Figure 3.1.1: Induced magnetization and currents in 3D bodies.

In any conducting permeable body placed in a magnetic field there are two types of induced magnetic moments. At zero frequency, dc, the incident magnetic field magnetizes the body so that it acquires a static magnetic dipole moment. This vector moment is in the direction of the inducing static magnetic field. As the frequency of the inducing field is increased a circulating current is induced to flow by virtue of Faraday's Law. This circulating current produces a magnetic moment, which is in the opposite direction to the 
inducing magnetic field. The net magnetic moment, that is responsible for producing the so called secondary fields measured away from the body, is a function of the frequency, conductivity, permeability, size and shape of the body. In this cartoon the induced principal magnetic moments are shown in black and the induced principal electromagnetic moments, for a particular frequency, caused by the induced currents are shown in red. The characteristics of these principal moments, and how they change with frequency, are the basis of shape and metal content determination. The magnetic and EM moments are always in opposite directions. The sphere has equal moments in any three orthogonal directions. The disk and cylinder have two identical minor axis moments and a different axial moment, and the flattened ellipsoid has three different moments. It is also important to see from this cartoon that the magnetic moments behave differently from the EM moments and can be large in directions where the EM moments are small. For example in the thin disk the axial magnetization will be very small whereas the axial moment will be the largest for EM induction. [This simplistic description for the disk is in fact only valid for very thin disks. For a disk of appreciable thickness the coplanar EM moments can be quite large due to the increase in induction number caused by the permeability. A relative permeability of 200 effectively increases the thickness by 200 and consequently presents a large cross-sectional area to the inducing electromagnetic field.] These observations clearly illustrate the need for broadband coverage not only to separate ferrous from nonferrous response but also to assist in relating the response to the shape of the body.

For regular bodies of revolution the induced moments are aligned with the symmetry axes of the object, and for a uniform inducing field they do not change direction with frequency. For an irregular object such as twisted scrap metal, the moment directions do change with frequency. For actual search systems the dimensions of the object may be comparable to the distance to, and size of, the transmitter and the moments may change size and direction as a function of frequency. In any event it is these induced moment characteristics that allow determination of the object parameters. To actually excite these moments it is clear that several polarizations of incident field are required.

A rigorous approach to the interpretation of the secondary fields measured with any particular search system would involve an inversion scheme to determine the parameters of an object that best reproduced the field data in a numerical simulation of the actual system. 
Forward modeling codes for solid metallic objects of arbitrary shape are only now becoming available, and for bodies of arbitrary relative permeability and arbitrary frequency they are slow even on very fast computers. It is certainly impractical to consider this approach for real time processing and interpretation in the field. Further, there are at the moment no modeling codes for ferrous and non-ferrous shells of various shapes. Since these are needed to represent typical UXO (there being little interest in detecting solid iron cannon balls) another approach is needed to represent the response of metallic objects.

For purposes of design, system evaluation and interpretation we have adopted a simple dipole moment representation of a target. This is basically the same approach used by Khadr et al. (1998), Bell et al. (1998), Pasion and Oldenburg (2001) and Baum (1999). With this approximation it is assumed that any target can be represented by three orthogonal dipole moments, the principal dipole moments (PDM) or, if normalized by the incident field, the principal dipole polarizations (PDP). We have concluded from these studies and our own simulators that a satisfactory approach to characterizing the target is to recover the PDM's and their directions as a function of frequency or time. The use of PDM's greatly simplifies the inversion process used to find the location and vector PDM's of the object. Moreover this representation is a practical way to characterize any object since it basically encapsulates the response in a compact manner. A rigorous identification will require matching of the PDM's, as a function of frequency or time, to a catalogue of objects whose PDM's have been predetermined.

Smith and Morrison (2004) describe an inversion algorithm, which locates an object and recovers its PDM for a specific search system in a given amount of system noise. The inversion process inherently assigns uncertainties to the extracted target parameters and location based on the uncertainties (noise) in the data. These uncertainties are the fundamental data used to assess the quality of the response and to estimate ROC curves. Smith et al. (2004b) used this inversion algorithm to determine the depths of detection, to a given uncertainty, and PDM's to a given uncertainty, of simple target spheres for several search configurations. This inversion analysis underlies the design methodology described in this section of the report. 


\subsection{Optimizing the transmitter-receiver configuration}

The inversion algorithm described in Smith and Morrison (2004), and used for system comparison by Smith et al. (2004b) is the fundamental tool used in this more generalized study. In a sense the approach used here can also be cast as an inverse problem: to determine the parameters of the AEM system that maximize the response for a given target subject to constraints on the system (size, platform, weight and power) and the anticipated ambient noise.

As with a rigorous inversion, the parameters of a proposed system are varied incrementally until a maximum response is obtained or until a maximum in the signal to noise ratio is obtained. For a given model and background, and with given system and ambient noise, the code is run repeatedly for changes in the system parameters until the variances in the estimated target parameters are minimized. This process provides an objective method for finding the optimum array configuration (i.e. the number of components and their spatial disposition, the transmitter moments and the bandwidth) needed to obtain the best estimate of the depth and vector principal moments of the target.

The first step in such a process can be seen in one of the examples shown in Smith et al. (2004b) (Figure 3.2.1). Here uncertainty estimates are used to compute the depths to which the PDM's and dipole locations can be estimated for steel spheres of varying radius, for two transmitter-receiver configurations. One meter square transmitter loops were used with a moment of $180 \mathrm{Amp}-\mathrm{m}^{2}$, and a receiver noise level of $1.97 \mathrm{nT} / \mathrm{s}$ in vertical field measurements, simulating an observed noise level, and $5.91 \mathrm{nT} / \mathrm{s}$ in horizontal field components (when present) simulating the larger noise levels observed in horizontal components. A step function turn-off transmitter current was used, as the most generic of waveforms, and an observation time of $610 \mu$ s after turnoff chosen to simulate the effective center time of the averaging gate of an existent commercial transmitter-receiver (T-R) system. For each radius sphere and for each T-R configuration the relative root mean squared (rms) inverted moment uncertainty and depth uncertainty were computed as a function of sphere depth, for spheres directly below the center of a 9 x 9 grid of system placements with $0.4 \mathrm{~m}$ spacing in $\mathrm{x}$ and $\mathrm{y}$. 

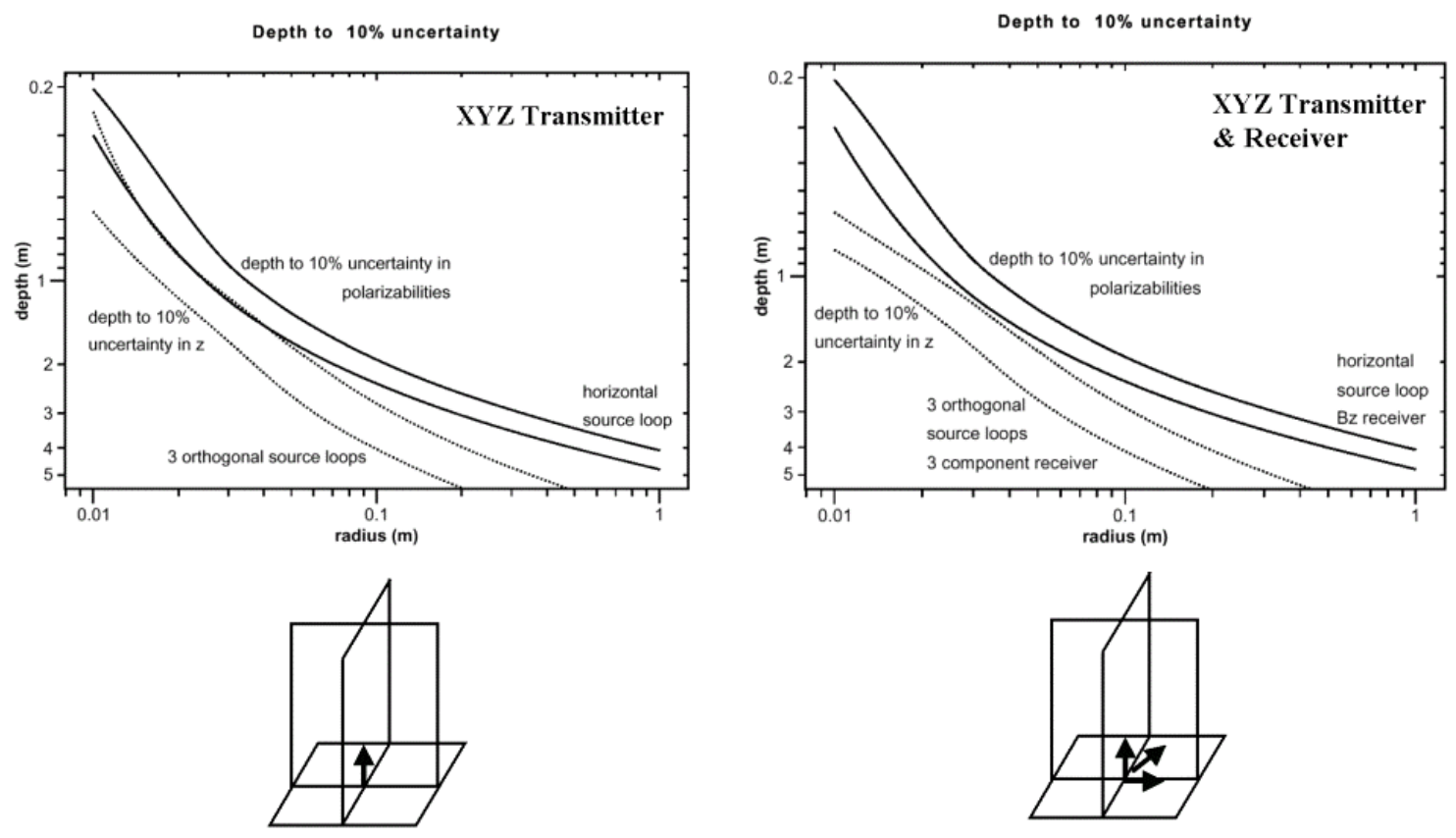

Figure 3.2.1: Depth to $10 \%$ polarizability uncertainty and $10 \%$ uncertainty in depth as a function of sphere radius for TxTyTz-Bz and TxTyTz - BxByBz systems.

The results, in Figure 3.2.1, are shown for an orthogonal three loop transmitter and vertical field, Bz, receiver and for the same transmitter with three orthogonal receivers. The results for the standard horizontal loop with a single vertical receiver are shown for reference as the solid line. The important role of multiple field polarizations at the target is easily seen in this figure. The depth of detection for a $10 \mathrm{~cm}$ radius sphere almost doubles with the three-component transmitter. But relatively little is gained by adding a triaxial receiver.

In general it was found that the object position can be estimated more precisely than the PDM's. The object position may be determined with only a single orientation of primary field, whereas estimating the full polarizability matrix requires illuminating the object with primary fields in at least three directions, each with a significant component in the direction orthogonal to the other two. Consequently, object depth can be resolved within $10 \%$ to greater depths than PDM's.

The recovery of the PDM's requires measurements using several polarizations of the incident field. How these are provided is a function of the spatial deployment of a system as well as the number of polarizations provided by different transmitters in the system. If a single horizontal loop source is moved in discrete intervals over an object then 
it is illuminated with different polarizations by virtue of its changing position in the source dipole field. This is illustrated in the cartoon of Figure 3.2.2.

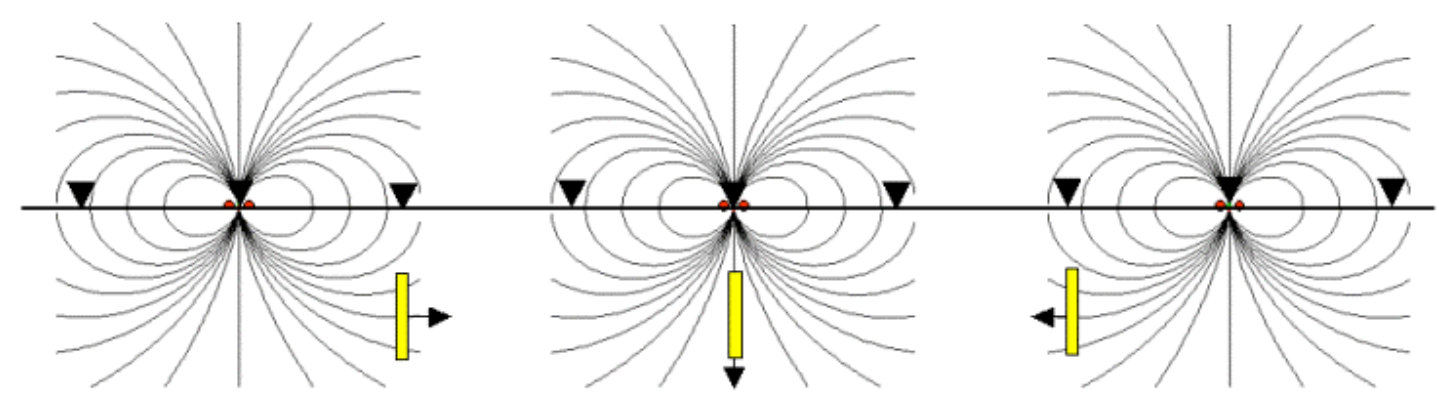

Figure 3.2.2

In the numerical experiments by Smith et al. (2004b) referred to above, the 'data' were in fact assumed to have been taken on a grid. An illustration of the effectiveness of an elementary system employing a single horizontal loop receiver within a horizontal loop transmitter is shown in Figure 3.2.3.
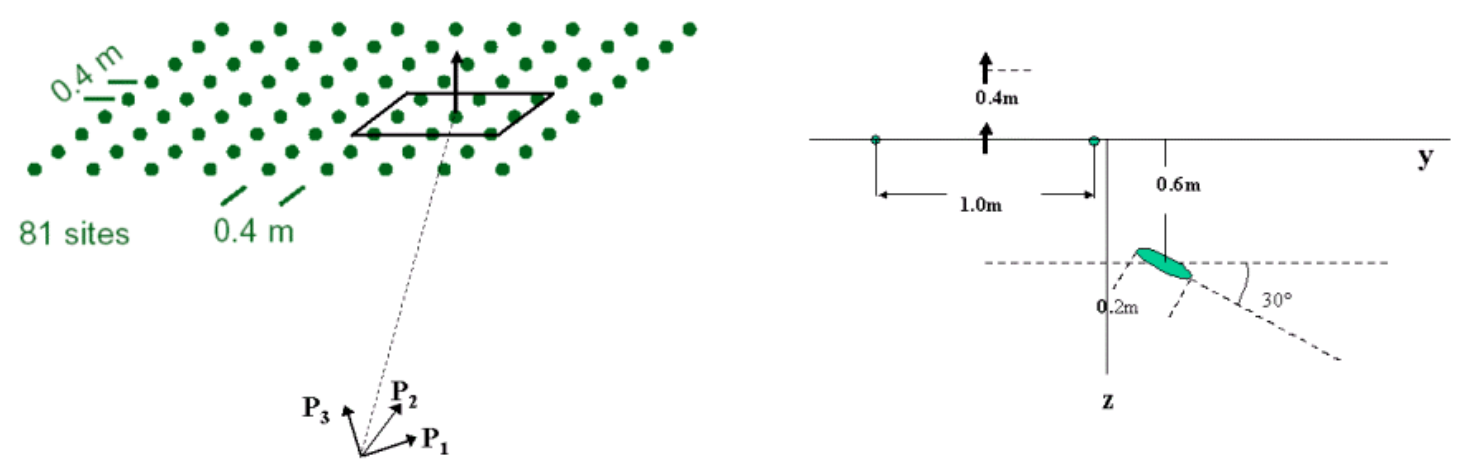

\begin{tabular}{|c|c|c|c|c|c|c|}
\hline & \multicolumn{3}{|c|}{ Model } & \multicolumn{3}{c|}{ Inversion } \\
\hline $\begin{array}{c}\text { Location } \\
(\mathrm{x}, \mathrm{y}, \mathrm{z})\end{array}$ & 0.2 & 0.2 & -0.6 & $0.207 \pm .008$ & $0.206 \pm .009$ & $-0.600 \pm .005$ \\
\hline $\begin{array}{c}\text { PDM } \\
\left(\mathrm{M}_{\mathrm{x}^{\prime}}, \mathrm{M}_{\left.\mathrm{y}^{\prime}, \mathrm{M}_{\mathrm{z}^{\prime}}\right)}\right.\end{array}$ & & & $0.785 \pm .023$ & $0.768 \pm .018$ & $0.529 \pm .011$ \\
\hline $\begin{array}{c}\text { Orientation } \\
\text { Vector } \\
\left(\mathrm{x}_{1}, \mathrm{x}_{2}, \mathrm{x}_{3}\right)\end{array}$ & 0 & 0.866 & -0.5 & $0.016 \pm .023$ & $0.850 \pm .026$ & $-0.526 \pm .043$ \\
\hline
\end{tabular}

Figure 3.2.3: Principal polarizations (PDM) and location for a dipping ellipsoid on 9 x 9 grid using a single horizontal loop receiver within a horizontal loop transmitter. 
The target is a dipping ellipsoid and the response is obtained on a 9 by 9 grid above it. The table shows that the principal polarizations (called PDM's in this figure) and location are very well recovered. In this inversion instrumentation noise was included but location errors were not. The accuracy of the inversion now depends on accuracy of the positioning on the grid. We have addressed the role of positioning error in this project and some examples are presented below. For rapid field surveys absolute positioning to the centimeter level might be expensive and difficult but relative positioning on the scale of the target depth might be easier to achieve. This suggests designing systems which are as close as possible to stand alone: multiple transmitters and a number of spaced apart receivers that locate and characterize the target from a single system set-up in the vicinity of the target. Such a system also suggests a search procedure involving two modes of operation. First, the object is located with a simple configuration and low power, narrow band, mode. Once located, the system switches to broadband, multicomponent mode and the accurate depths and PDM's, and identification through the look-up catalog of object parameters. Finally, anticipating the results presented below, a system comprising multiple receivers will require sensors that are smaller than the loop receivers currently employed. In this report we analyze a variety of system configurations that are optimum for detecting objects and for determining their PDM's while minimizing their complexity and minimizing the number of sensors required.

The general simulation/inversion code was used to investigate the number of receivers, and their orientations, required for an optimum measurement of the principal polarizabilities and depths. In general, it was found that the object position can be estimated more precisely than the PDM's for the same object depth. The object position may be determined with only a single orientation of primary field, whereas estimating the full polarizability matrix requires illuminating the object with primary fields in at least three directions, each with a significant component in the direction orthogonal to the other two. Consequently, object depth can be resolved within 10\% to greater depths than PDM's with the same uncertainty. The following brief description and examples are taken from a comprehensive analysis of this problem that is discussed in more detail in the paper by Smith et al. (2005) that has been submitted for publication. 
In this analysis, transmitter systems are comprised of one or several rectangular loops of fixed size, and a number of receiver coils approximated as point measurements. Three families of designs are presented: a) systems for use on a 2-D grid of positions with negligible error in relative instrument location, b) systems for use on a line of positions with negligible error in relative instrument location, and c) systems for stand alone use, insensitive to instrument positioning errors.

The general approach is summarized with reference to Figure 3.2.4 for a 2D grid of data. The color plot shows the uncertainty in the determination of the principal polarizabilities for a test object (in this case a sphere for ease of illustration) of various diameters. The data for the inversion are the responses for the simple in-loop configuration (similar to the commercial EM 61 system) at 81 positions of the $9 \times 9$ grid shown on the right. The contours are drawn at locations in the vertical section beneath the array where the uncertainty in polarizability is equal to the polarizability itself - in effect a signal to noise ratio of one. As mentioned above the depth can be determined with any specified uncertainty at greater depth than the polarizability. Using Figure 3.2.1 as a guide, the depth of a $60 \mathrm{~mm}$ sphere can be determined to within $10 \%$ at a depth $50 \%$ greater than depth at which the polarizability is determined to within $10 \%$. Consequently the contours in plots like those of Figure 3.2.1 can be used as rough measures of the depth of detection of the relevant object. For example the polarizability of a $40 \mathrm{~mm}$ sphere could be determined with an uncertainty of less than its polarizability anywhere in the section above the contour labeled $40 \mathrm{~mm}$ but the depth would be well determined below this contour. The $40 \mathrm{~mm}$ sphere would consequently be well located anywhere to a depth of about one meter and in a swath of plus or minus $1.0 \mathrm{~m}$ around its horizontal position. In comparing configurations for a specific target, the 'best' are those for which the contours for that target's diameter are deepest in the section and flattest. We have found this graphic presentation to be an excellent way to illustrate the range and accuracy of a particular configuration of transmitter and receiver. 


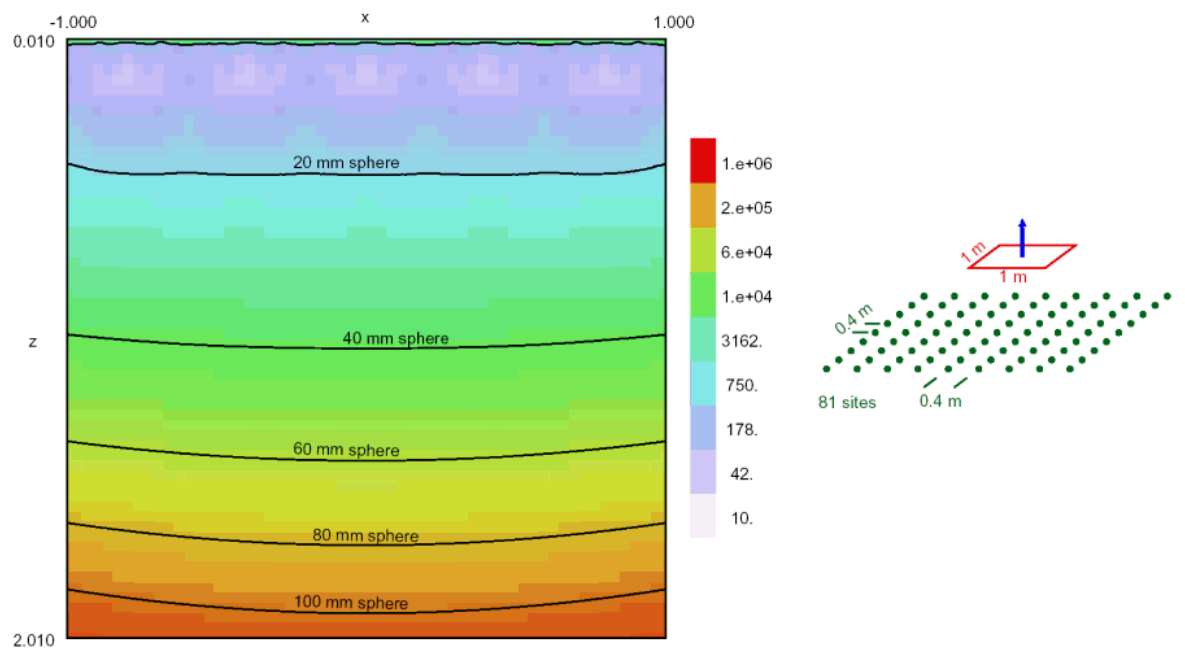

Figure 3.2.4: Rms uncertainty in polarizability as a function of position on $9 \times 9$ grid using simple in-loop configuration.

The improvement in detectability that accrues from using multiple transmitters and receivers is evident in the results shown in Figure 3.2.5 for a configuration with 3 orthogonal transmitters and 3 orthogonal receivers deployed on the same $9 \times 9$ grid. The detectability of the $40 \mathrm{~mm}$ sphere is pushed down to $1.5 \mathrm{~m}$ and the $80 \mathrm{~mm}$ sphere, previously detectable to $1.5 \mathrm{~m}$ is now pushed down, off the graph, to more than $2.0 \mathrm{~m}$.

Next, in Figure 3.2.6, the uncertainty in polarizability is plotted for a simple two transmitter three receiver (vertical) configuration deployed at 21 positions along a line over the target. In this and subsequent multi receiver configurations the inversion code was used to determine the location of the receivers that optimized the resulting uncertainty plot. We found that the orientation of the sensors was less important than the number used and their spacing. Since we have observed that ambient EM noise fields are predominantly in the horizontal plane, we have elected to use vertical receivers in the array studies. Further, to minimize the footprint of the system we imposed penalties on the inversion for receivers outside the perimeter of the horizontal transmitter.

The results for the line system indicate that the depth range is reduced a little and the swath width is considerably reduced over the grid system of Figure 3.2.5. Nevertheless it is important to note that comparable results would be obtained if successive lines were run with line spacing of $0.5 \mathrm{~m}$. The grid after all could be considered as multiple parallel lines with spacing of only $0.4 \mathrm{~m}$. If survey cost is proportional to the number of readings 
then there are appreciable savings in the multi-element line profiling. We will return later to the role of position uncertainty in this assessment.
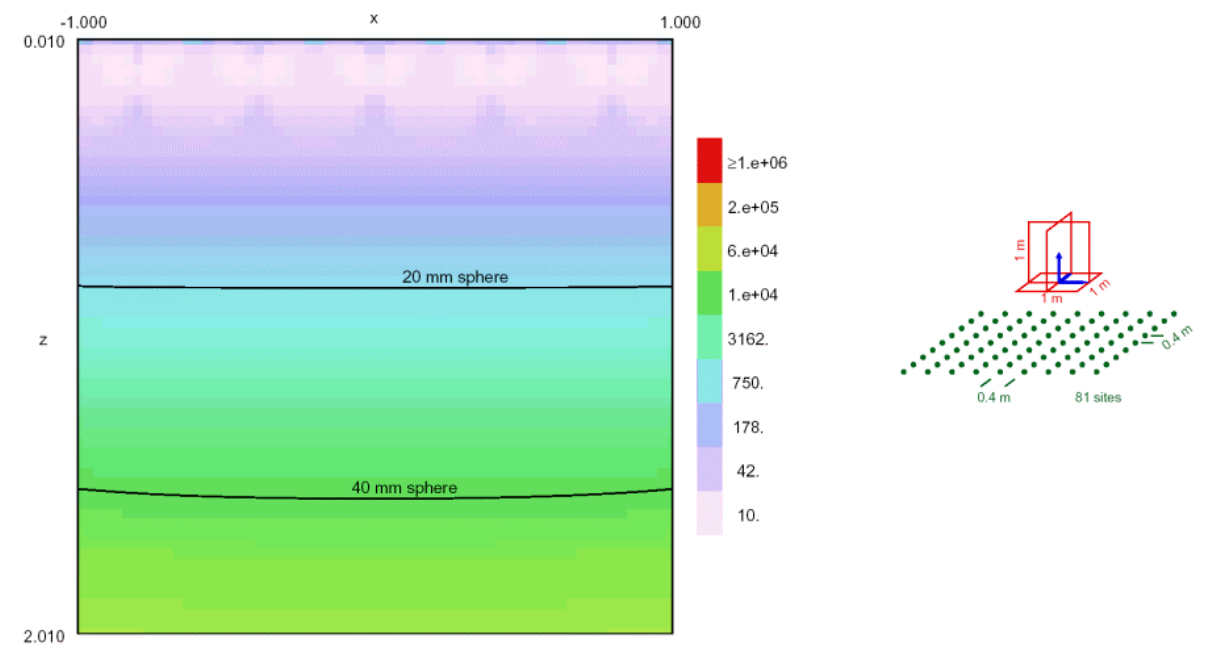

Figure 3.2.5: Rms uncertainty in polarizability as a function of position on $9 \times 9$ grid using 3 orthogonal transmitters and 3 orthogonal receivers.
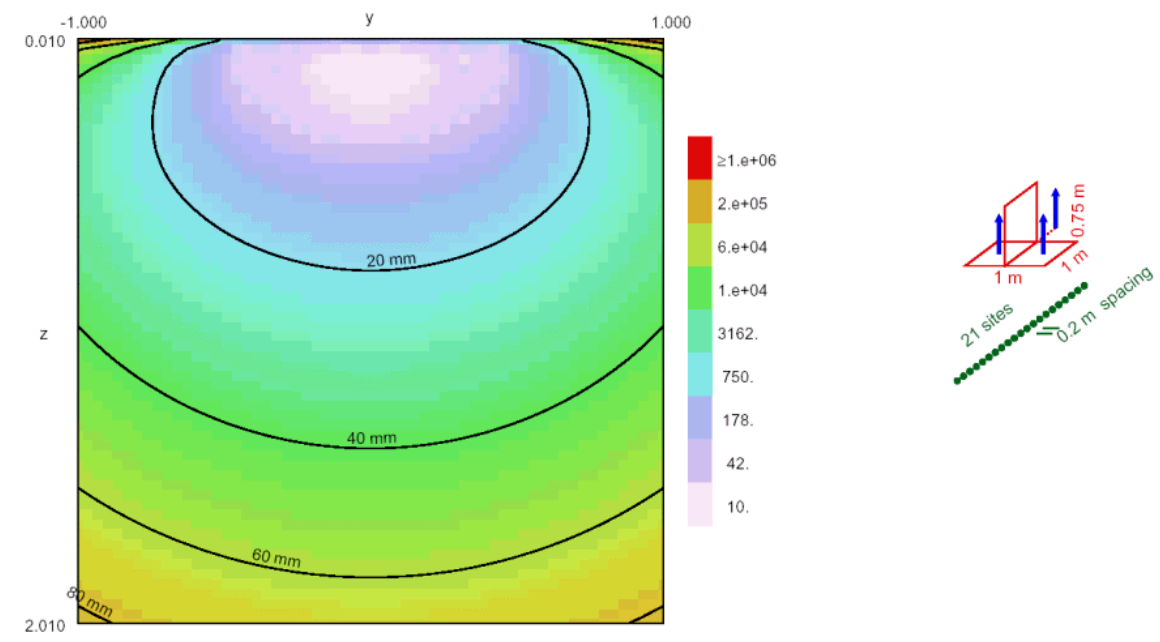

Figure 3.2.6: Rms uncertainty in polarizability as a function of position using two transmitters and three receivers in a profile mode.

Finally we experimented with single or stand alone configurations which could determine depth and polarizations from a single position in the vicinity of the target. In all these systems we used a three component transmitter. The results for a four receiver configuration are shown in Figure 3.2.7. The depth of detection is reduced somewhat and the pattern is narrowed and skewed. Another important measure of the design process is illustrated for this array in the plot of Figure 3.2.8. Here the uncertainty in polarizability is 
plotted as a function of orientation of an elongate target at a depth of one meter. The plot clearly shows that there are 'blind spots' for this configuration - orientations for which the number of T-R pairs is inadequate to determine the orientation. Smith et al. (2005) have determined theoretically that $13 \mathrm{~T}-\mathrm{R}$ pairs are required to determine the polarizabilities so the 12 pairs are in fact not enough. Adding a fifth receiver, in the optimized five element array of Figure 3.2.9, makes a dramatic improvement in the orientation determination. Even at a depth of $1.6 \mathrm{~m}$ there are no blind spots for this array as seen in Figure 3.2.10, although there is only a modest improvement in the depth of detectability, Figure 3.2.9.
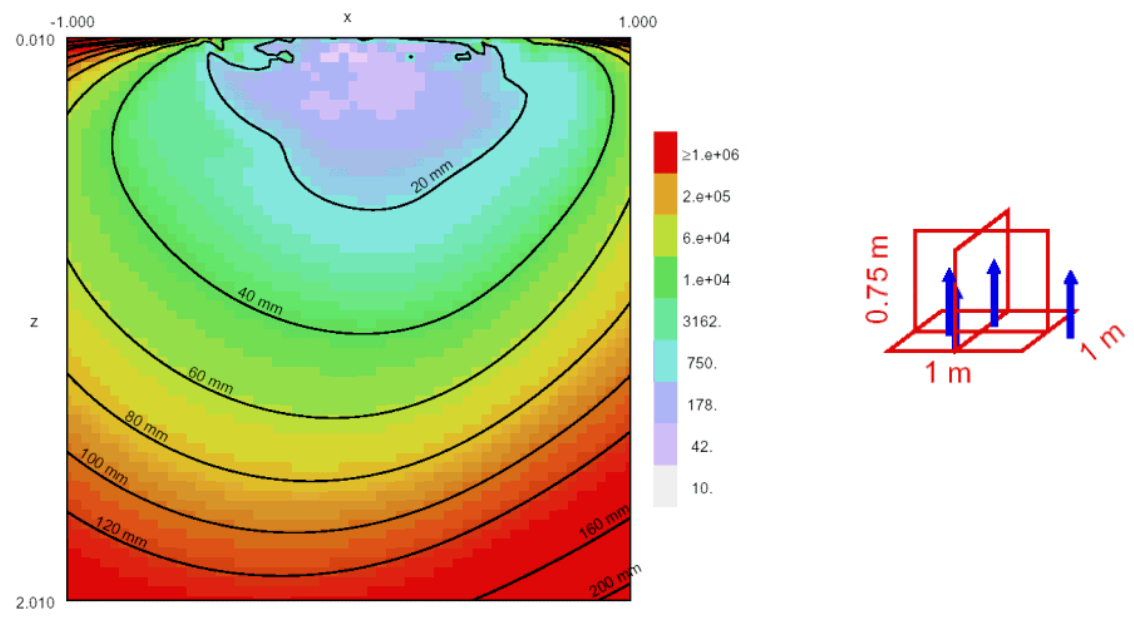

Figure 3.2.7: Rms uncertainty in polarizability as a function of position using three transmitters and four receivers in a stand alone mode.
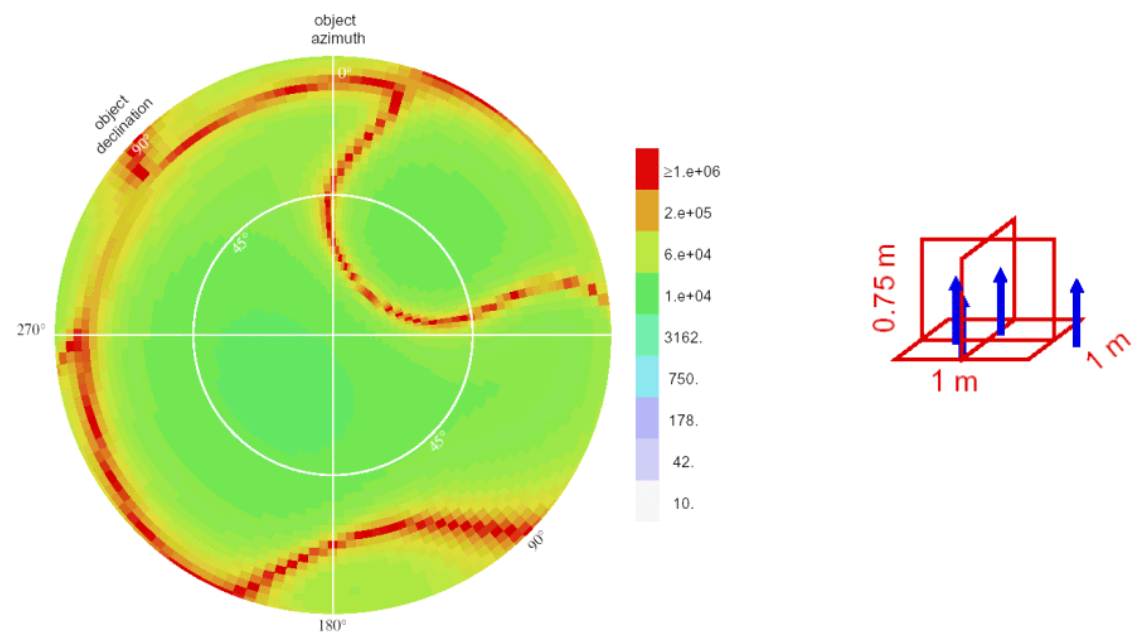

Figure 3.2.8: Rms uncertainty in polarizability as a function of orientation of an elongate target at a depth of $1 \mathrm{~m}$. 

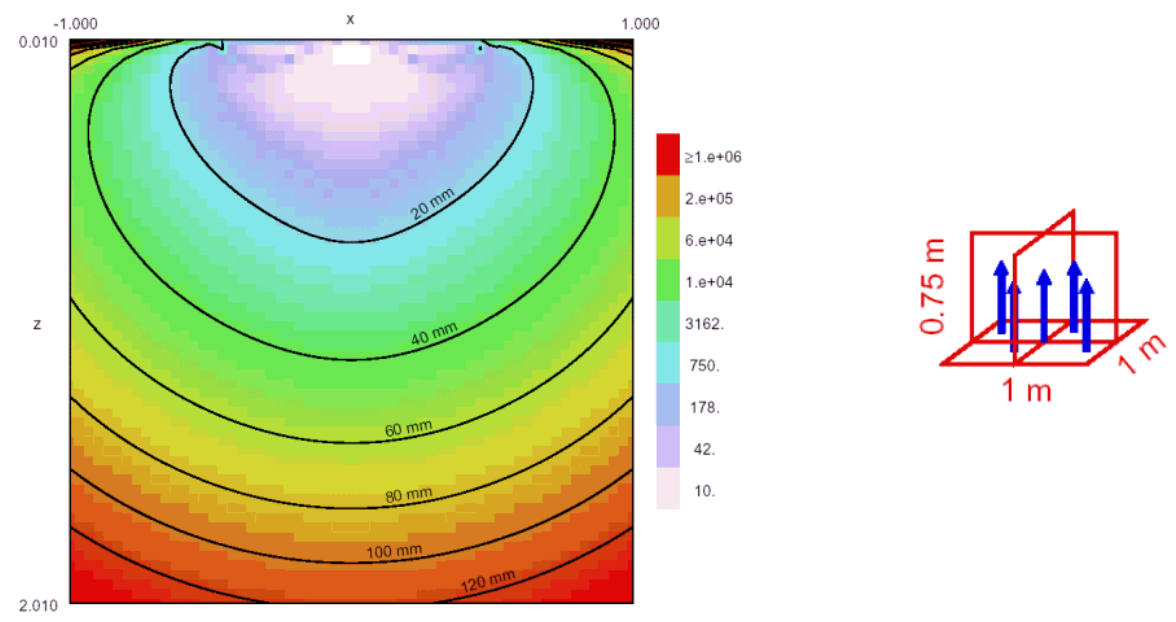

Figure 3.2.9: Rms uncertainty in polarizability as a function of position using three transmitters and five receivers in a stand alone mode.
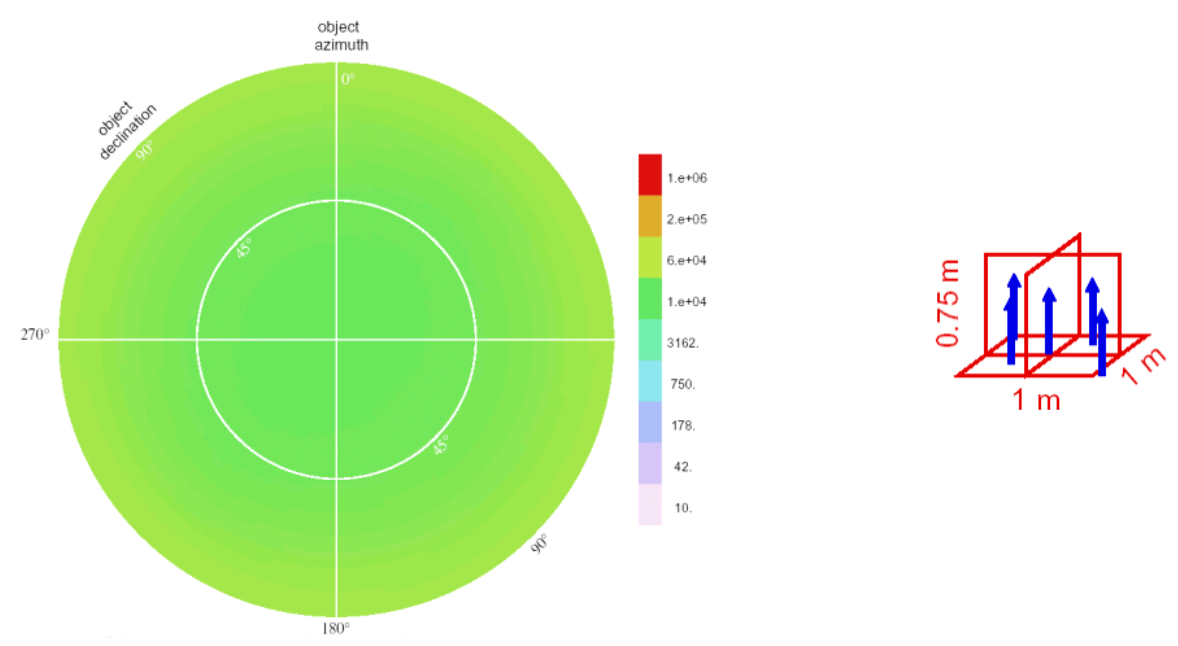

Figure 3.2.10: Rms uncertainty in polarizability as a function of orientation of a target at $1.6 \mathrm{~m}$ depth using three transmitters and five receivers in a stand alone mode.

Allowing the inversion code to 'choose' the location of the vertical receivers results in a rather irregular pattern as shown on the right of Figure 3.2.9 as an example. From a fabrication viewpoint it might be simpler to arrange the sensors in a regular pattern dictated by the frame or structure of the transmitters. We tried the regular pattern of Figure 3.2.11 and found very little reduction in detectability compared to the optimum pattern. The conclusion of studies such as this is that optimization is not a strong function of variation of sensor position around the optimum one. 

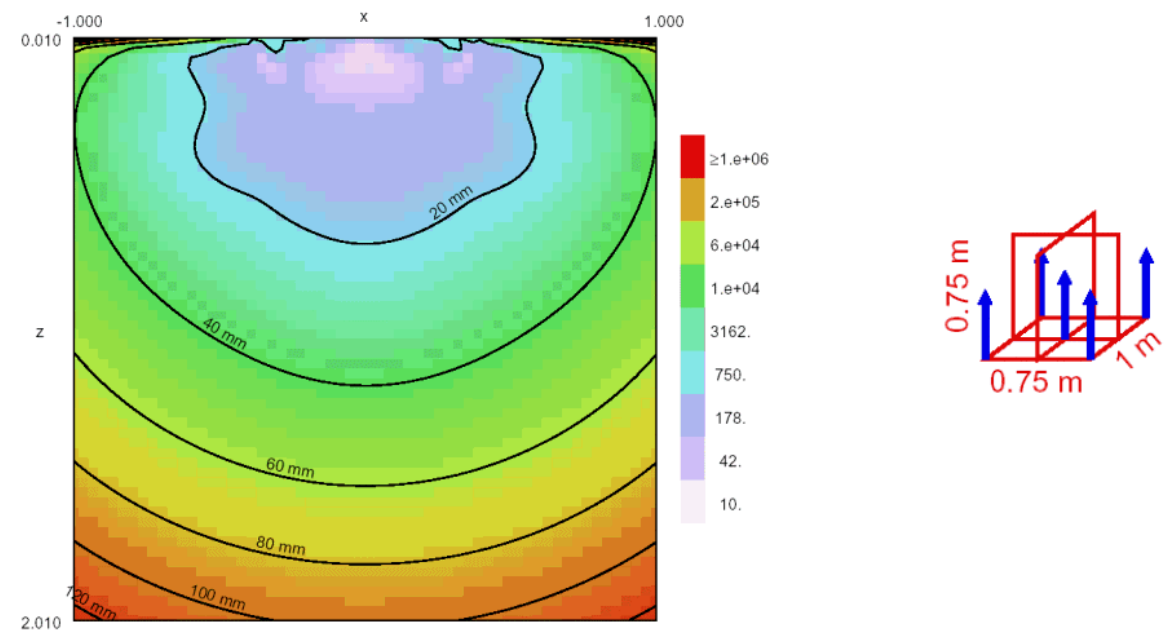

Figure 3.2.11: Rms uncertainty in polarizability as a function of position using three transmitters and five receivers in a regular pattern dictated by transmitter frame.

By using an ingenious combination of horizontal loops one can provide both a vertical and horizontal polarization of the incident field at a target beneath the array. In Figure 3.2.12 the horizontal loops consist of four independent rectangular loops and a redundancy of 9 vertical receivers. When two adjacent loops are energized with the same, say clockwise, current flow they sum to produce an equivalent large loop of the same polarity which produces a primary field directed vertically beneath the center of the loop. When energized with opposing current flows a multipole field is produced with field lines that are horizontal beneath the loops. Similar energization of the orthogonal pair of loops produces the requisite third, horizontal, polarization of the field incident on the target.

The results (Figure 3.2.12), even with 9 receivers, are not as good as those obtained with the transmitter consisting of three orthogonal loops and 5 receivers, Figure 3.2.11.

Finally, to summarize these illustrative examples, it is important to note that the depth of detectability for the 3 transmitter 5 receiver stand alone system, Figure 2.2.11, is almost as good as the 3 transmitter 3 receiver system when the latter is deployed at 21 positions along a line or profile. It is also almost as good as the current industry standard EM61 system when the latter is deployed on a grid of 81 points, Figure 3.2.4. On the other hand it is not nearly as good as the 3 transmitter 3 receiver configuration deployed on the same grid, Figure 3.2.5. 

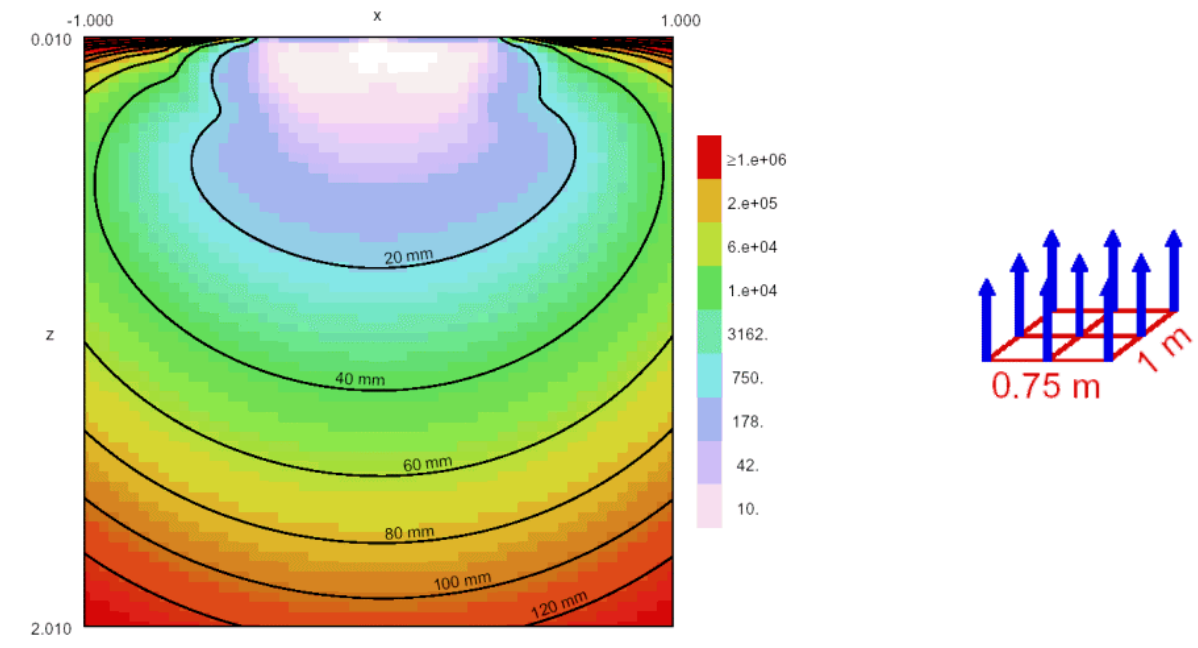

Figure 3.2.12: Rms uncertainty in polarizability as a function of position using four independent horizontal loops and 9 vertical receivers.

The results to date were obtained assuming that there were no errors in the position of the system on the grid or along the line. In a practical survey there are such errors, largest in the absolute position on the grid, less in relative position along a line segment and zero for the stand alone system (the latter finds the polarizability and the target location relative to the T-R system so the uncertainties are proportional only to the system noise). Figures 3.2.13 and 3.2.14 display the relative uncertainties in polarizability and depth respectively as a function of the position error for the three deployment arrays shown on the right of the figures. The statistics were created by repeated inversions with random misplacements of each measurement point by the indicated position error. The target in this experiment was a horizontal $22 \mathrm{~mm}$ shell with an aspect ratio of 8:1 at a depth of 0.75 m. The relative uncertainties in polarizability and depth for both the line and grid configurations remain less than that of the stand alone system (about 5\%) as long as the position uncertainty is less than about $2 \mathrm{~cm}$. Relative GPS positioning is at best a few $\mathrm{cm}$ so without expensive and logistically difficult laser or microwave positioning it appears that the stand alone system is superior to line and grid deployments. But the stand alone system can also be operated in line or grid mode the results of which would be significantly better than any of the individual schemes discussed till now. Since the stand alone system has the same footprint as the best current commercial system, the field operational issues are identical to the current systems but with vastly improved detection and identification 
properties. Further the system retains excellent detection and polarization determination from irregularly located single positions, which may be all that is available in terrain with obstacles to regular grids or profiles.
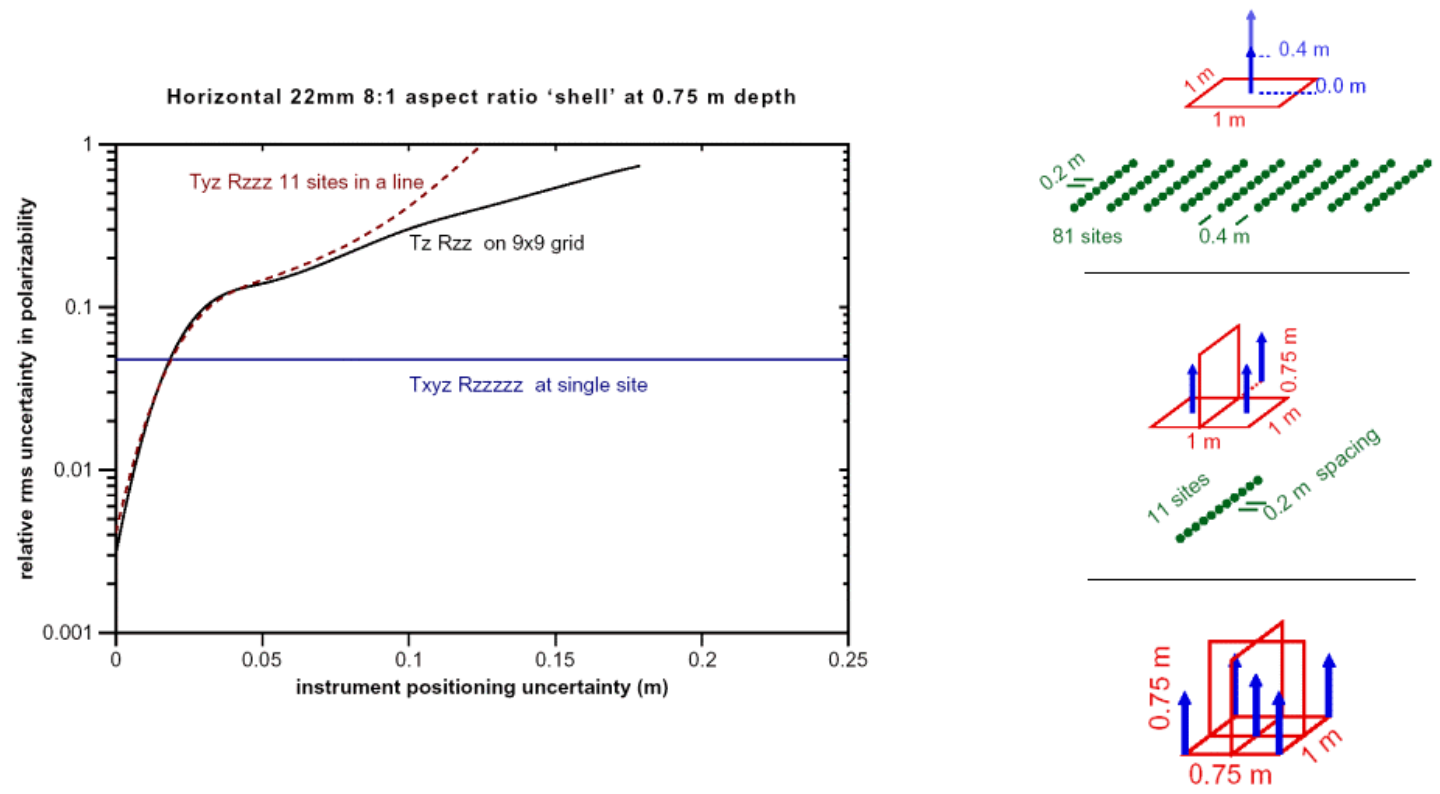

Figure 3.2.13: Relative rms polarizability uncertainty as a function of instrument location error for $22 \mathrm{~mm} \mathrm{8:1} \mathrm{aspect} \mathrm{ratio} \mathrm{shell} \mathrm{at} 0.75 \mathrm{~m}$ depth at $610 \mu$ s after transmitter shutoff.
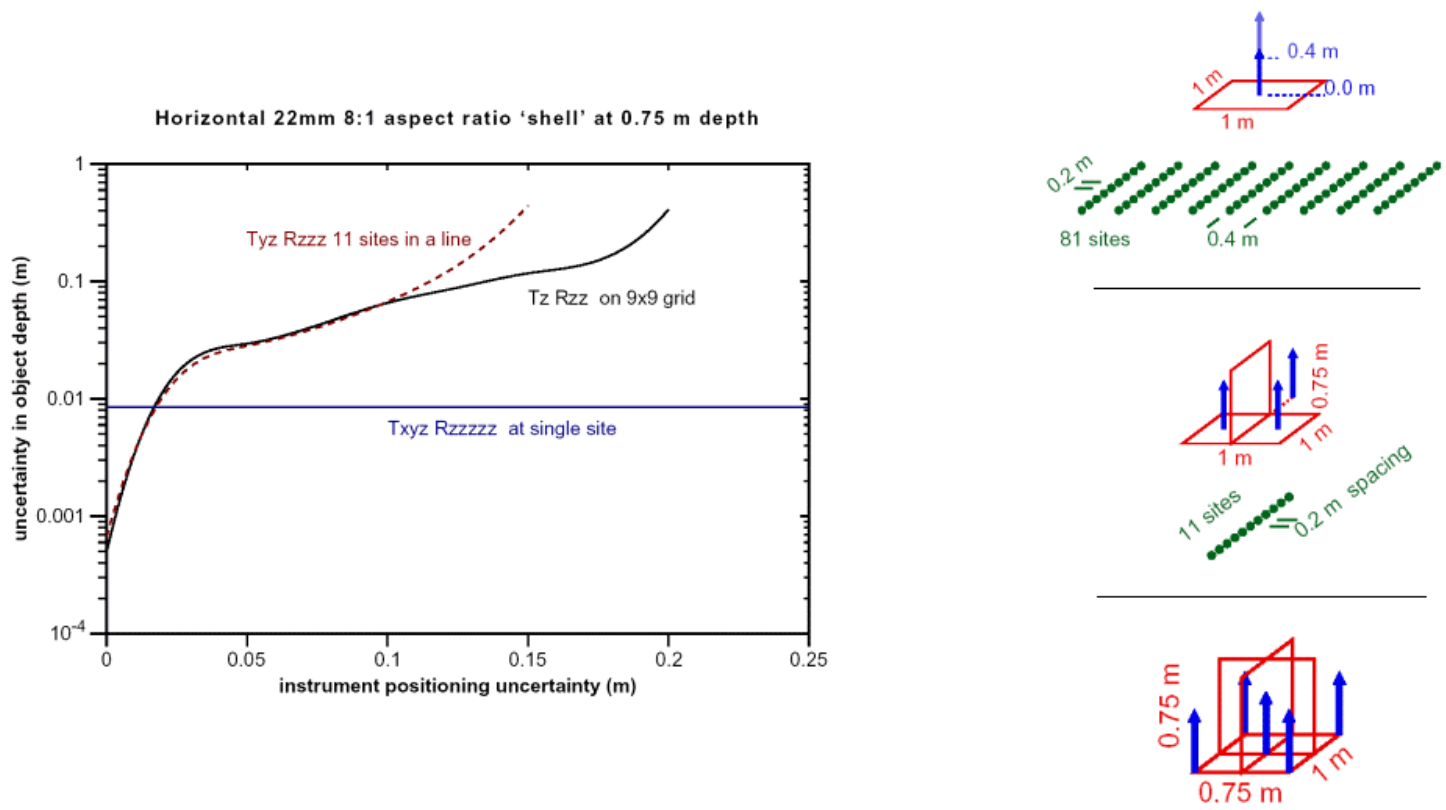

Figure 3.2.14: Relative rms depth uncertainty as a function of instrument location error for $22 \mathrm{~mm} \mathrm{8:1} \mathrm{aspect} \mathrm{ratio} \mathrm{shell} \mathrm{at} 0.75 \mathrm{~m}$ depth at $610 \mu \mathrm{s}$ after transmitter shut-off. 
Given the position and polarizability matrix of an object, it is straightforward to calculate estimation uncertainties in polarizabilities and coordinates that would arise from detection with given transmitter and receiver configurations with specified source current and receiver noise levels. One can compare performance of prospective systems using a simple statistic such as the total summed squared uncertainty in polarizability estimates for on object at a specific position relative to the detection system, or sum the squared polarizability uncertainties for test objects at a range of positions ('control points') to construct a somewhat more general measure of performance. Since uncertainties typically vary greatly with the depth of an object below a system, it makes sense to include objects at number of depths in the sum, and to weight them differently according to depth, for example, using the weight function $\mathrm{w}^{2}(\mathrm{z})=\left(\mathrm{z} / \mathrm{z}_{\max }\right)^{-7}$ which results in roughly equal contributions from shallow and deep objects.

As conceiving and comparing a large number of prospective configurations is a potentially burdensome task, we have automated the procedure, in effect, using general non-linear optimization methods to minimize the weighted summed squares of polarizability uncertainties for prospective objects over a range of depths below the system. For simplicity, we keep the transmitter configuration fixed; for example, three orthogonal $1 \mathrm{~m}^{2}$ rectangular loop transmitters abutting the horizontal plane $(\mathrm{z}=0)$, with centers at horizontal position $\mathrm{x}=\mathrm{y}=0$, and allow receiver positions (and possibly orientations) to vary for a fixed number of receivers. To model the typically larger amount of noise in horizontal field measurements compared to vertical field measurements we treat receiver noise as three times greater in horizontal than vertical receiver orientations with a sinusoidal variation in between.

In general, we find that for spherical objects (three equal principal polarizabilities) receiver position and orientation optimization results in all receivers being oriented vertically. Optimization is simplest for spherical test objects as spheres are rotationally invariant. Polarizability estimation uncertainties do depend on the polarizability matrix. Computing summed squared polarizability uncertainty for elongate test objects (with only a single non-zero principal polarizability in their limiting case) one can compare for uncertainties for elongate objects of any orientation. Doing this for systems with four, five, or six receivers optimized for spherical targets, one finds that four receiver systems 
have a continuous series of elongate object orientations for which the summed squared uncertainty is extremely large (or unbounded). For five receiver systems, a few elongate object orientations remain with extremely large uncertainties. And for six receivers the problem is greatly mitigated, but still one finds, for example, some horizontal orientations result in rms polarizability uncertainties 50 times greater than for the same elongate object in a near vertical orientation.

In performing the same sort of optimization for non-spherical objects, one has the difficulty that polarizability uncertainty depends on object orientation. A quick method to partially stem this difficulty is to simply sum squared polarizability uncertainties over the test object in a number of standard orientations at each of the control points at which uncertainties are computed for the test object, for example, orienting the object major axis along directions corresponding to the face centers and corners of a cube centered at the origin. For example, doing this in a six-receiver optimization for elongate objects over a range of test depths from $0.2 \mathrm{~m}$ to $1.6 \mathrm{~m}$, reduces the variation in rms polarizability uncertainty with orientation for objects at $1 \mathrm{~m}$ depth from the factor of 50 to a factor of 5 , with a 6.5 reduction of the largest rms polarizability uncertainty. This analysis is reported in detail in Smith et al. (2005).

\section{Worst-case optimization}

Summing squared uncertainties over estimates for a number of orientations of an elongate object is a simple ad hoc method of adapting the minimization objective to find receiver arrays which perform better for elongate objects than systems which have only been optimized for resolving spheres. Being ad hoc, it leaves some uncertainty as to whether there might be other objects with polarizabilities that would be unresolvable, despite having sizable polarizabilities.

To first order in receiver noise level, for a given transmitter-receiver system and object position and orientation, the polarizability uncertainties are independent of the scale of the polarizability matrix (Smith and Morrison, 2004); the polarizability uncertainties for an object with small polarizabilities and for a similar object with large polarizabilities with the same orientation and the same ratios of principal polarizabilities are the same. Of course, for an object with small polarizabilities, the relative 
uncertainties in the polarizabilities are much greater than the relative uncertainties for the similar object with larger polarizabilities. So, in optimizing a system, one need not worry about the scale of the polarizability matrix for which it is optimized.

Ostensibly, one might like to find systems that are optimal in a mini-max sense: giving the minimum summed squared polarizability uncertainty for whatever polarizability object has the greatest summed squared uncertainty. In general, as we consider both object polarizability and position as parameters to be estimated, uncertainties in one and the other are not independent, so a mini-max problem for uncertainties in one separate from the other is not convenient. For mini-max minimization, instead of considering the summed squared uncertainty in all components, it is much more convenient to worry about only the squared uncertainty in the component that is least well constrained by a set of data. In summing over squared uncertainties, this component would make the greatest contribution to the summed squared uncertainty. Given the structure of the sensitivity matrix (Jacobian) of data with respect to polarizabilities and position, as it depends on object polarizability, we find the worst-case polarizability matrix, which has the component with the largest uncertainty of all components of all polarizability matrices. In most cases examined, the worst case polarizabilities have one principal polarizability that is less than one hundredth of the largest principal polarizability, and a second principal polarizability that is less one tenth of the largest principal polarizability.

For a given receiver-transmitter system and object location, once the worst case polarizability is in hand, it is straightforward to compute its summed squared polarizability uncertainty, and to use this summed over prospective object positions (control points) as an objective function in non-linear optimization. More details are given in Smith and Morrison (2005b).

\subsection{Spectral properties of target response}

The analysis till now has concentrated on the detection of a target, and the determination of depth and principal polarizabilities of the target. In the introduction we also described the vital step of measuring the polarizabilities as a function of frequency, or time, to determine the shape, size, metal content and even the wall thickness of the object. 
Only the variation of the induced moments with frequency permit the determination of the various shapes shown in the cartoon of Figure 3.1.1. This analysis immediately raises the issue of the necessary bandwidth. To get an idea of the bandwidth over which the principal polarizabilities undergo their defining variation we again turned to the simulator to find the spectral and transient responses for some typical UXO.

The response of a target is defined here as the secondary field (B) at a given receiver for an incident field from a given transmitter. The response is thus a function of the T-R pair as well as the properties of the target. Induction coil sensors typically measure the time derivative of the secondary field $\mathrm{B}$ so the $\mathrm{dB} / \mathrm{dt}$ response is often used.

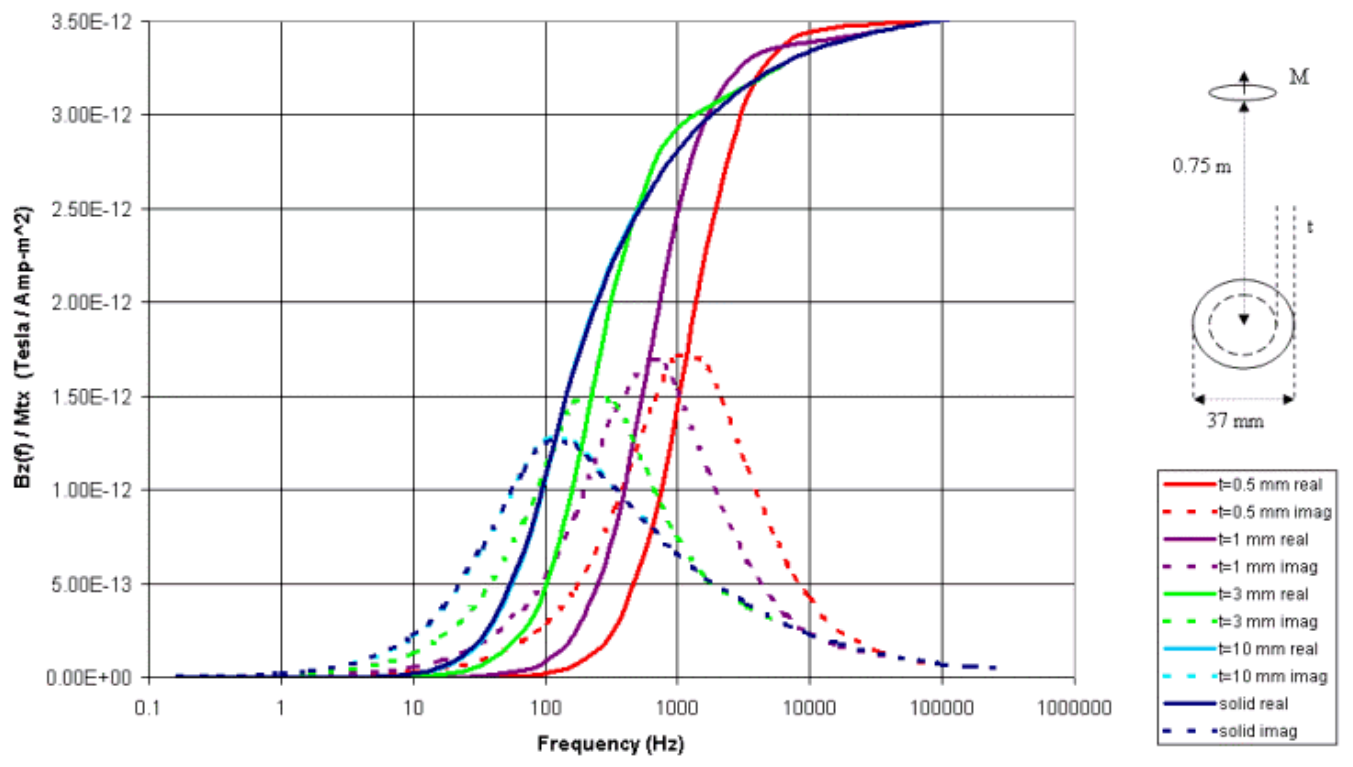

Figure 3.3.1: Normalized secondary fields (real and imaginary components) as a function of frequency for $37 \mathrm{~mm}$ aluminum spherical shell of various thicknesses at $0.75 \mathrm{~m}$ depth.

To illustrate the spectral response of a variety of targets, we have chosen to use a simple horizontal loop transmitter with an in-loop vertical receiver deployed directly above the target. This is basically the model for the EM61 commercial system. This configuration, the target size and shape, and the separation of the T-R system from the target are shown to the right of the response plots in the following figures. In all cases the secondary fields in nano-Tesla (nT) are normalized by the transmitter moment. We have plotted the frequency response real (or in-phase) and imaginary (out of phase or 
quadrature) and the transient response for $\mathrm{B}$ and $\mathrm{dB} / \mathrm{dt}$ to illustrate diagnostic behaviors in both domains. We have studied the response of non-magnetic conductors as well as the more common permeable conductors and for the simple sphere we have analyzed the effect of changing shell thickness on the response.

The first analysis shows the effect of shell thickness on the frequency response of an aluminum $37 \mathrm{~mm}$ spherical shell $0.75 \mathrm{~m}$ below the T-R pair. For any body the high frequency response, so called inductive limit response, depends only on the size. In the frequency domain, variations in the shell thickness produce characteristic changes in real and quadrature response at frequencies in the decade above and below the frequency of peak quadrature response. In Figure 3.3.1 this band is between 30 and $3000 \mathrm{~Hz}$. The high frequency could be estimated at a frequency of $10 \mathrm{kHz}$ so an ideal bandwidth for identifying this target would be $30 \mathrm{~Hz}$ to $10 \mathrm{kHz}$ or 2.5 decades.

In the time domain, Figure 3.3.2, the step function response in B clearly resolves thicknesses from the early time asymptote at $3 \times 10^{-5} \mathrm{sec}$ (which also determines the size) to about $3 \times 10^{-3} \mathrm{sec}$. The thinnest shell has a pure exponential decay while the solid sphere has a 'stretched' response becoming exponential only beyond $10^{-3} \mathrm{sec}$. The sensor need only have a dynamic range of 1.5 decades to resolve the response.

The time derivative $\mathrm{dB} / \mathrm{dt}$, measured by standard induction coil sensors, is shown in Figure 3.3.3. Diagnostic changes in the response occur between $3 \times 10^{-6}$ and $10^{-2} \mathrm{sec}-\mathrm{a}$ much wider bandwidth than that for B and a dynamic range of at lest 2.5 decades is required to define the responses. The time rate of change $\mathrm{dB} / \mathrm{dt}$ can be measured in units of Tesla/sec. A more convenient unit of $\mathrm{dB} / \mathrm{dt}$ is the Volt $/ \mathrm{m}^{2}$. It can be shown directly from the Maxwell equations that $1 \mathrm{Volt} / \mathrm{m}^{2}=1 \mathrm{Tesla} / \mathrm{sec}$ exactly. 


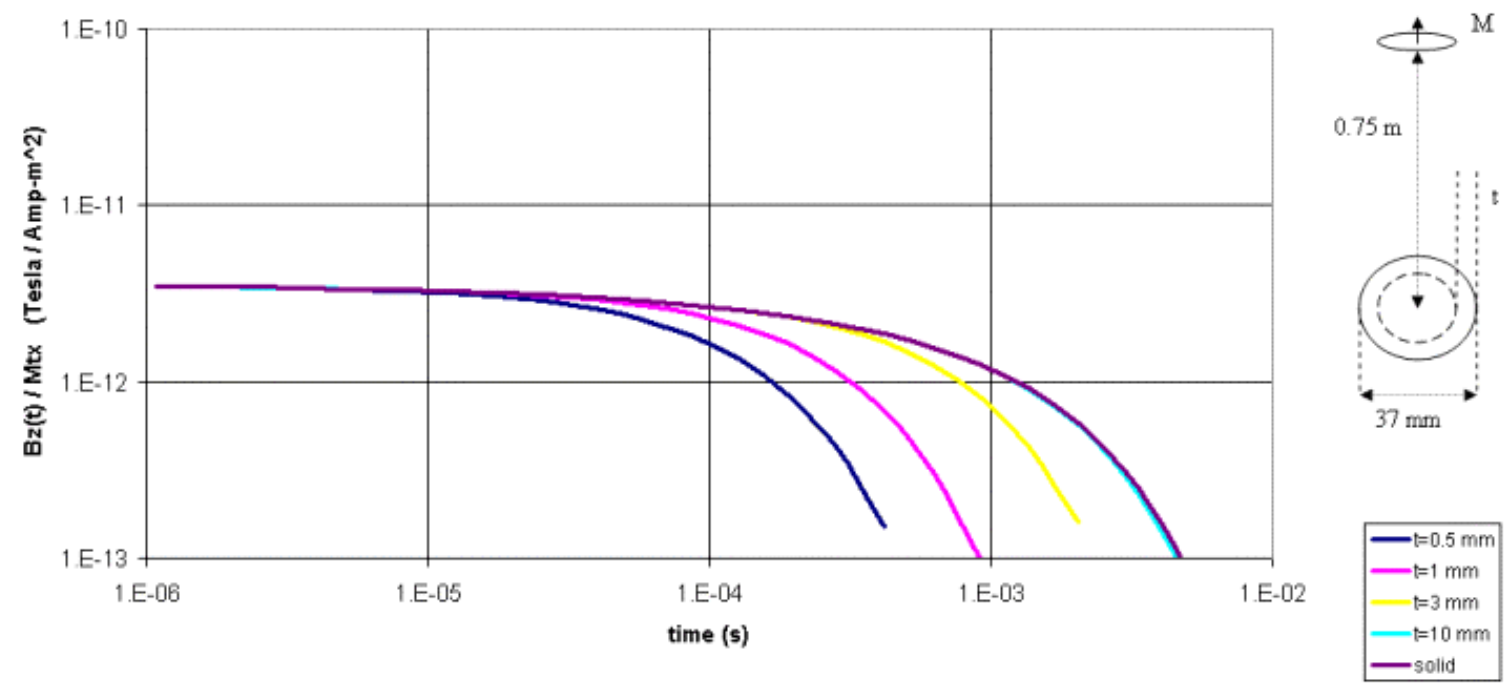

Figure 3.3.2: Normalized magnetic field response as a function of time for $37 \mathrm{~mm}$ aluminum spherical shell of various thicknesses at $0.75 \mathrm{~m}$ depth.
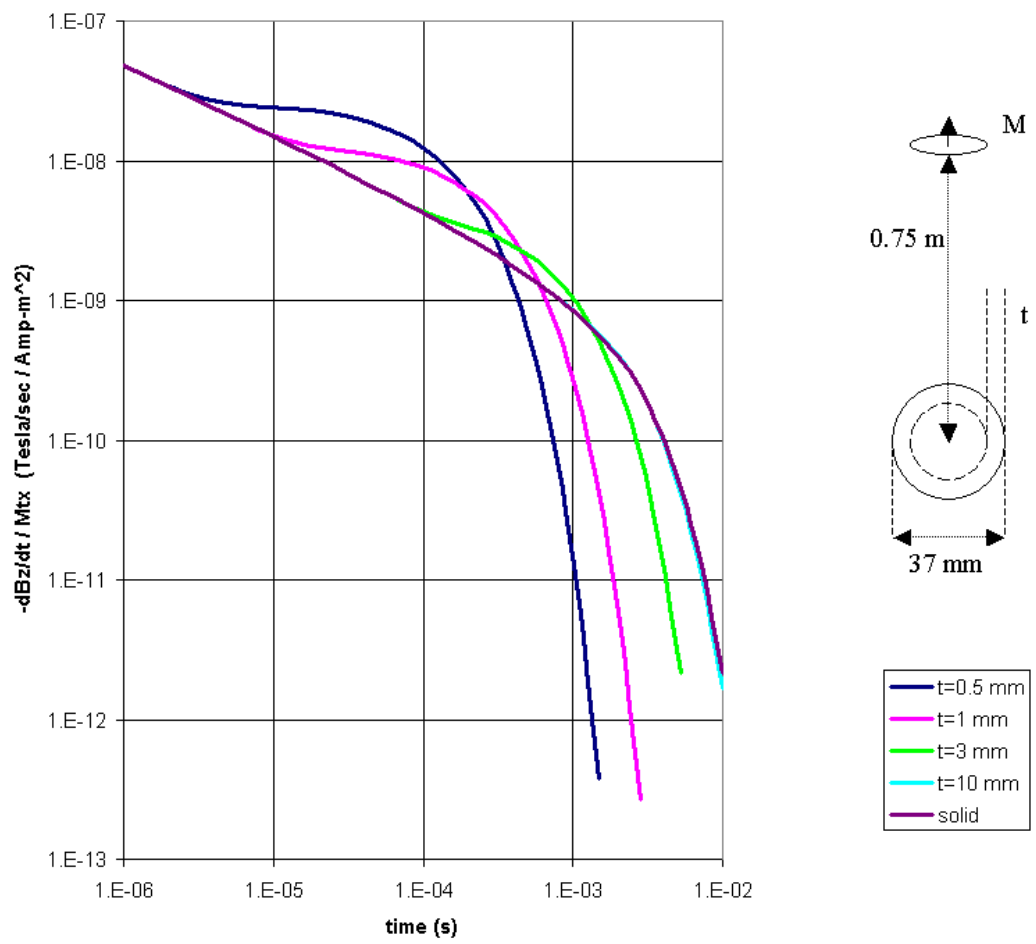

Figure 3.3.3: Normalized $\mathrm{dB} / \mathrm{dt}$ response as a function of time for $37 \mathrm{~mm}$ aluminum spherical shell of various thicknesses at $0.75 \mathrm{~m}$ depth.

The frequency domain response for the same spherical shells but with a magnetic permeability of 200 is shown in Figure 3.3.4. The permeability introduces the opposing static magnetization which drives the real response negative at low frequencies and causes 
small perturbations in the quadrature response also at low frequencies. Resolution of shell thickness for a magnetic target is only possible at frequencies below about $300 \mathrm{~Hz}$. This simple model provides the first evidence that it may be difficult if not impossible to resolve shell thickness in magnetic targets in the frequency domain.

The prospect is improved considerably in the time domain for B as shown in Figure 3.3.5. As expected, the thickness variations are manifested at late time, between $10^{-5} \mathrm{sec}$ and $10^{-3} \mathrm{sec}$. The thinnest shell develops an exponential decay by $3 \times 10^{-5} \mathrm{sec}$ while the solid hasn't become exponential by $10^{-3} \mathrm{sec}$. The intervening thicknesses could be well resolved with a dynamic range of 2 decades. The resolution of shell thickness with $\mathrm{dB} / \mathrm{dt}$ is markedly less than with B, Figure 3.3.6. Curve separation is clear over only one decade of time, $10^{-3}$ to $10^{-2} \mathrm{sec}$, and over 3 decades of amplitude but the diagnostic separation only begins after over two decades of amplitude decay from shut-off.
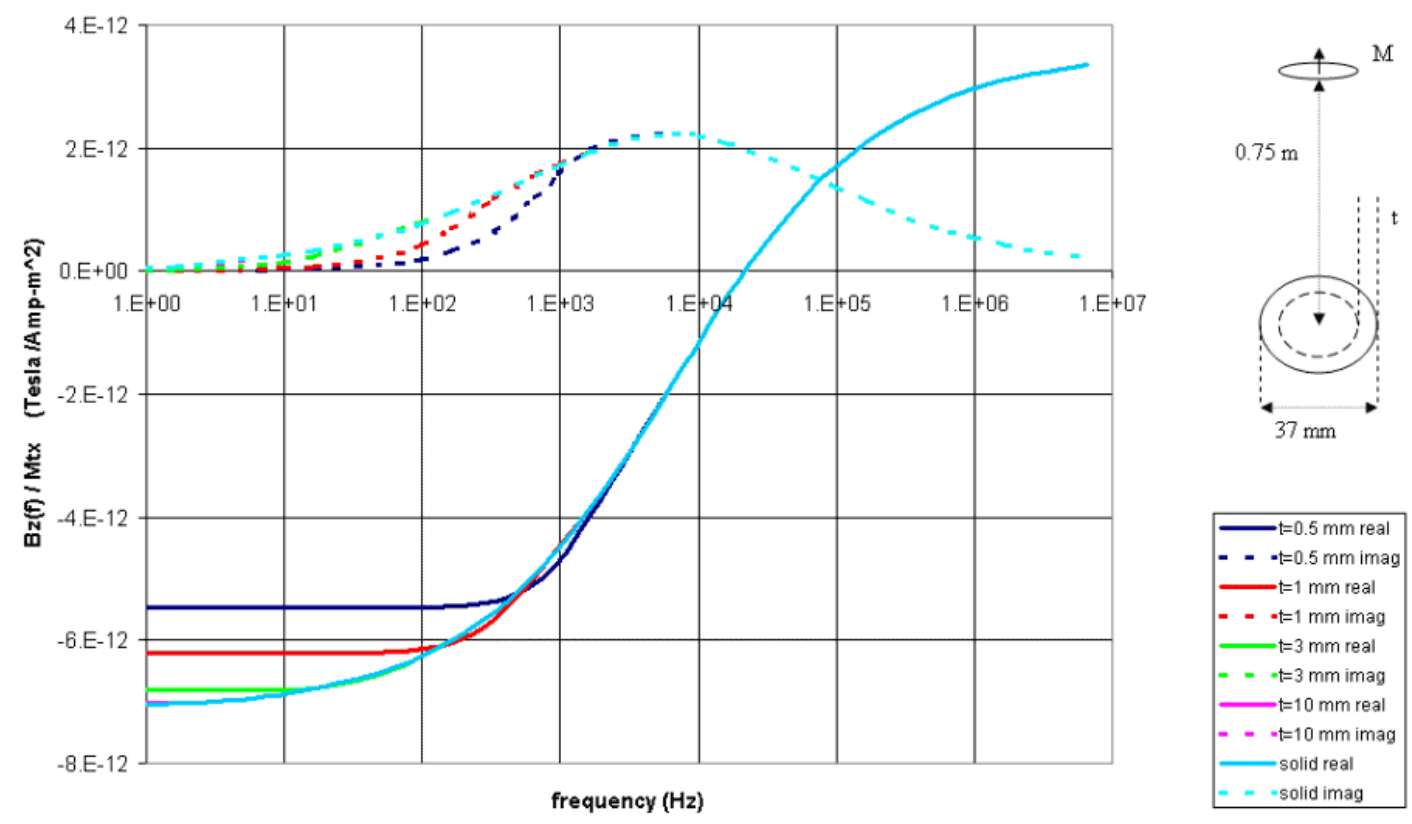

Figure 3.3.4: Normalized magnetic field response as a function of frequency for $37 \mathrm{~mm}$ magnetic spherical shell of various thicknesses at $0.75 \mathrm{~m}$ depth. 


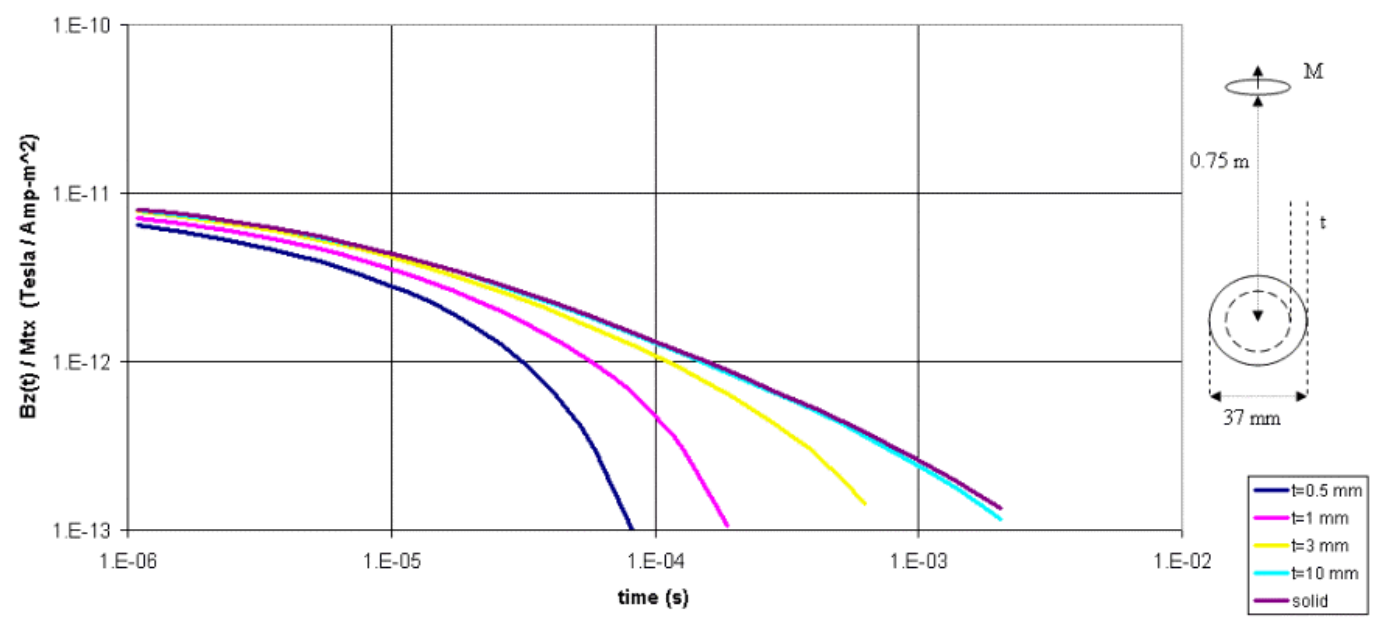

Figure 3.3.5: Normalized magnetic field response as a function of time for $37 \mathrm{~mm}$ magnetic spherical shell of various thicknesses at $0.75 \mathrm{~m}$ depth.
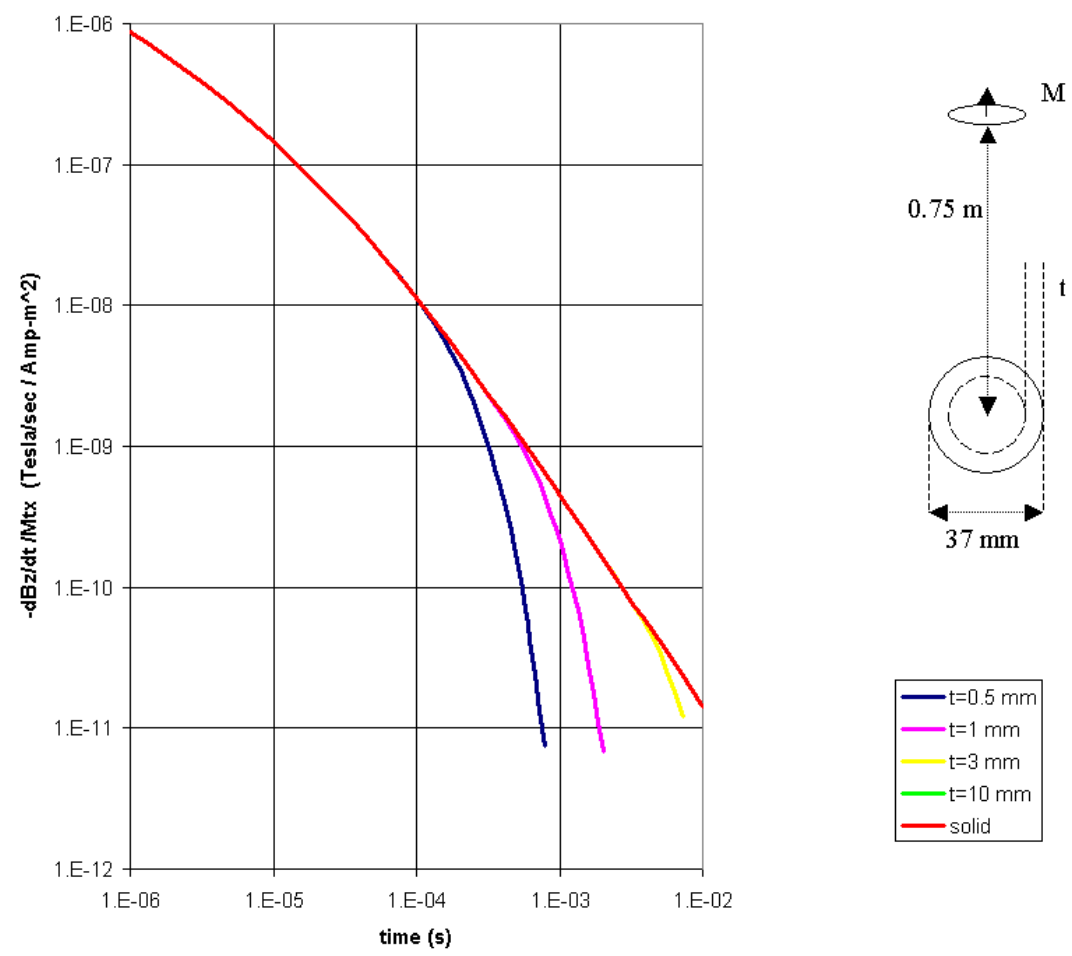

Figure 3.3.6: Normalized $\mathrm{dB} / \mathrm{dt}$ response as a function of time for $37 \mathrm{~mm}$ magnetic spherical shell of various thicknesses at $0.75 \mathrm{~m}$ depth.

These sample models illustrate some general practical conclusion: B rather than $\mathrm{dB} / \mathrm{dt}$ is more diagnostic of shell thickness for magnetic and non-magnetic objects, and shell thickness of magnetic objects is difficult to resolve in the frequency domain. 
The general analysis for optimizing the T-R configuration in Section 3.2 used idealized dipoles (spheres) as the target. It is important to know how well these results apply to the case of actual, usually elongate, UXO. In Figure 3.3.7 we have shown the simulated results for an EM61 style in-loop system over a typical $37 \mathrm{~mm}$ shell $0.75 \mathrm{~m}$ below the system. The shell is solid, steel, and has an aspect ratio (length/diameter) of 3:1. The plot shows the $\mathrm{dB} / \mathrm{dt}$ response for both the horizontal and vertical orientation of the shell. For comparison the response of the $37 \mathrm{~mm}$ sphere is included. It is immediately evident that the actual shell response, for both orientations, is larger than the sphere responses. It may be concluded that all the detectability analyses of Section 3.2 are worse case scenarios and so are excellent design guides for a working system.

In Figure 3.3.7, and subsequent plots for other targets, we have also plotted the response from the conductive ground in which the target is immersed. The responses for two ground resistivities, 10 and $100 \mathrm{Ohm}-\mathrm{m}$, are plotted. The ground response basically imposes an early time limit on the time window available for target discrimination. Once the target response falls below the ground response it will be poorly resolved, especially since the ground response itself will variable due to the inhomogeneous nature of the near surface. (The role of magnetic ground is still being analyzed and will be described in a later report.) For a conservative design approach we assume a ground of $10 \mathrm{Ohm}-\mathrm{m}$.

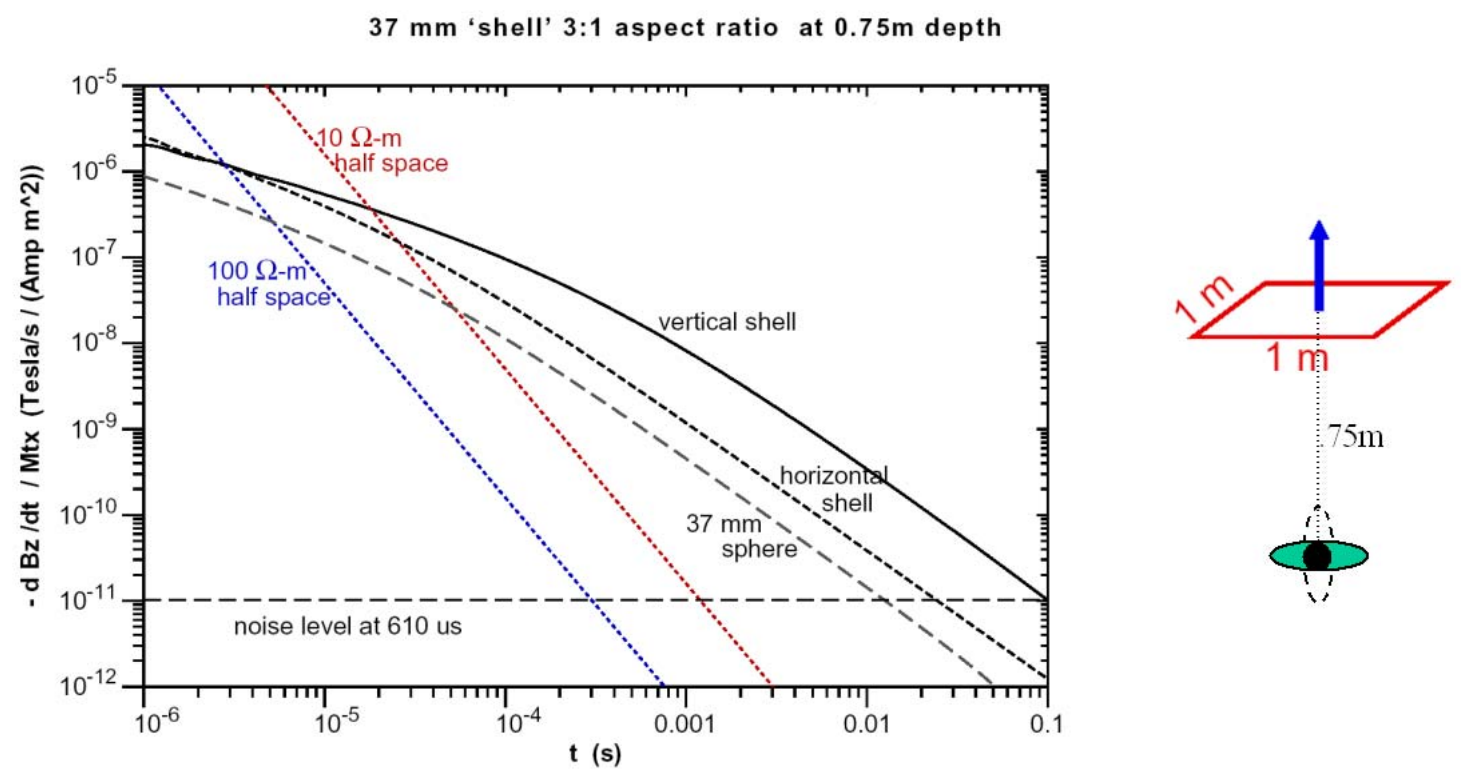

Figure 3.3.7: Amplitude of $\mathrm{dB} / \mathrm{dt}$ response for $37 \mathrm{~mm}$ sphere, horizontal and vertical shells $3: 1$ aspect ratio at the depth of $0.75 \mathrm{~m}$ as a function of time together with responses for $10 \Omega$-m and $100 \Omega$-m half-space. 
For this EM61 simulation we can also include the normalized practical noise level for this system at $610 \mu \mathrm{sec}$. Since the noise level is not known at other times into the transient, we have simply assumed that the noise is constant at this level for all times and plotted this horizontal noise floor accordingly.

The early time limit imposed by the ground and the later time limit imposed by the noise sets the time window over which the response can be measured. For this target the window is roughly from $2 \times 10^{-5}$ to $3 \times 10^{-2} \mathrm{sec}$ (for the horizontal shell). Referring back to Figure 3.3.6, it can be seen that this window is adequate to define the size (early time asymptote) of the sphere and to resolve its thickness, but the dynamic range is almost five decades above the noise floor. This may be difficult to achieve in practice. Using only the later time data, say from $2 \times 10^{-4} \mathrm{sec}$, may be satisfactory especially since it should be noted that the orientation has already been determined in the first stage of the inversion.

The response of this elongate target also reveals the fact that the step response is different for the different polarizabilities of a non-spherical target. The responses of the horizontal and vertical orientations of this $37 \mathrm{~mm}$ shell actually cross at a few microseconds, the horizontal shell giving a longer response at very early time than the vertical shell. This is an excellent illustration of the fact that the ratio of the polarizabilities is not the same as the geometric aspect ratio of the body. As can be seen in Figure 3.3.7 the ratio of the horizontal and vertical responses at later time is 10:1. (An interesting observation is that the ratio of the horizontal responses for the target to that of a sphere of the same diameter is 3:1. This appears to hold true for all the elongated targets we considered. The result holds for the $\mathrm{B}$ response as well.)

For $\mathrm{B}$ rather than $\mathrm{dB} / \mathrm{dt}$ the window where the target response exceeds the ground response widens to $3 \times 10^{-6} \mathrm{sec}$ at the low end, Figure 3.3.8. Since there are no existing systems that measure $\mathrm{B}$ we have no estimate of when the late time response meets the noise floor. However, the horizontal and vertical responses still differ by a factor 10 and most importantly the observed separation (including very early time) is confined to less than 3 decades of amplitude variation. Instrumentally this is much more manageable than the much higher dynamic range required for a $\mathrm{dB} / \mathrm{dt}$ system. 


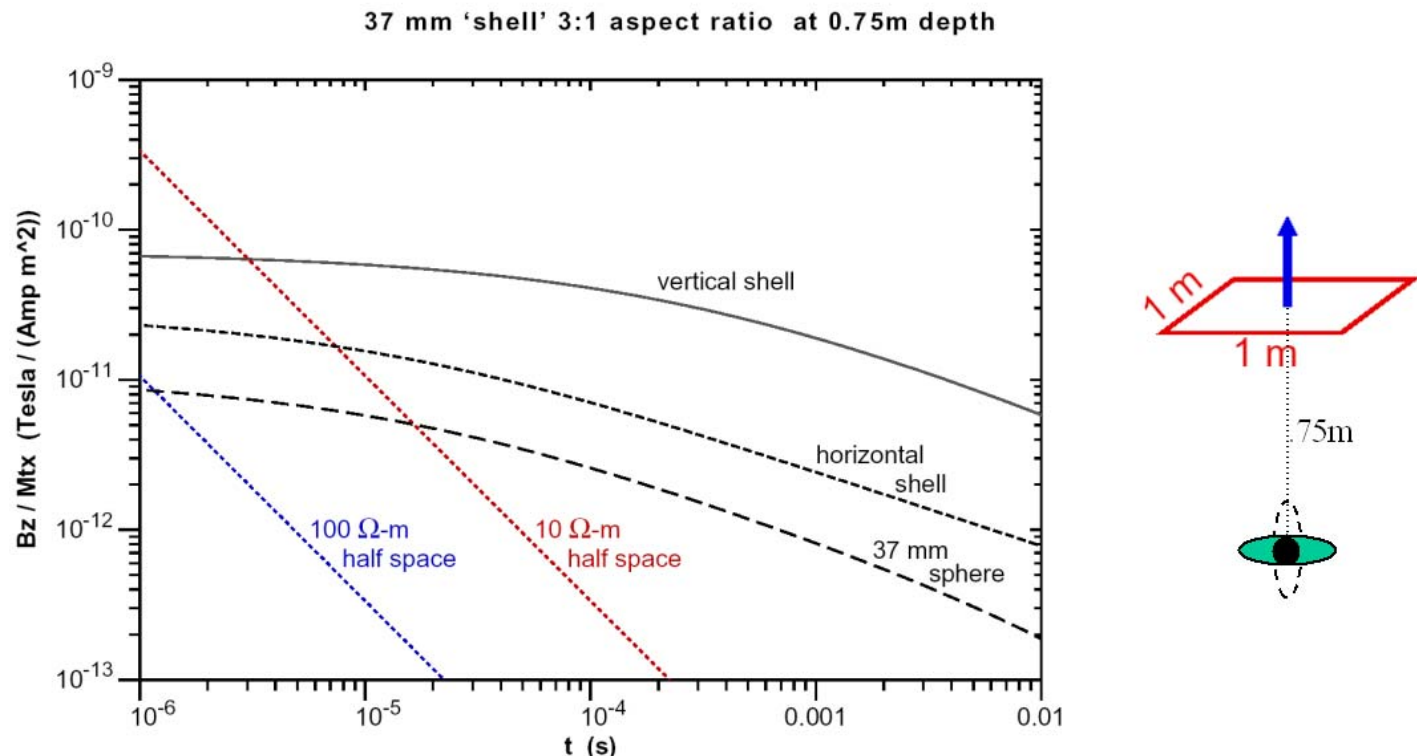

Figure 3.3.8: Amplitude of magnetic field response for $37 \mathrm{~mm}$ sphere, horizontal and vertical shells $3: 1$ aspect ratio at the depth of $0.75 \mathrm{~m}$ as a function of time together with responses for $10 \Omega$-m and $100 \Omega$-m half-space.

Figures 3.3.9 through 3.3.14 show the $B$ and $\mathrm{dB} / \mathrm{dt}$ responses for three other targets representative of the extremes of UXO to be characterized - a $22 \mathrm{~mm}$ shell with an aspect ratio of 8:1 (Figures 3.3.9 and 3.3.10) at a depth of $0.75 \mathrm{~m}$, a $105 \mathrm{~mm}$ shell with an aspect ratio of $4: 1$ at a depth of $2.65 \mathrm{~m}$ (Figures 3.3.11 and 3.3.12), and a larger $155 \mathrm{~m}$ shell with an aspect ratio of 4.4:1 at a depth of $4.55 \mathrm{~m}$ (Figure 3.3.13 and 3.3.14). In all these simulations the ground response limits the early time response to microseconds in B and 10 's of microseconds in $\mathrm{dB} / \mathrm{dt}$. The dynamic range requirement is reduced in $\mathrm{B}$ and responses to at least $0.01 \mathrm{sec}(10 \mathrm{msec})$ are required to clearly resolve the decay characteristics. For the large target at greater depth the response must be obtained to at least $100 \mathrm{msec}$ at which point, at least for this sample EM61-like system, the response in $\mathrm{dB} / \mathrm{dt}$ is below the noise level (see Figure 3.3.13). 
$22 \mathrm{~mm}$ 'shell' $8: 1$ aspect ratio at $0.75 \mathrm{~m}$ depth
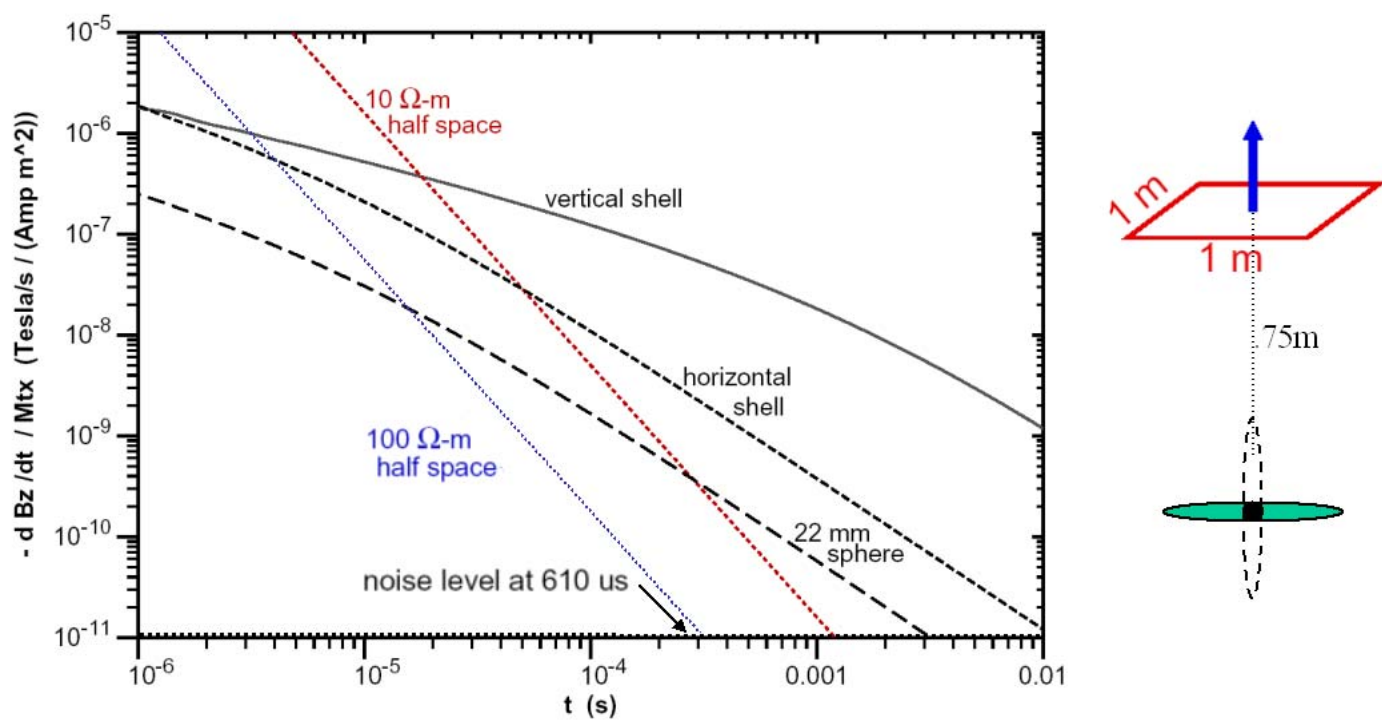

Figure 3.3.9: Amplitude of $\mathrm{dB} / \mathrm{dt}$ response for $22 \mathrm{~mm}$ sphere, horizontal and vertical shells $8: 1$ aspect ratio at the depth of $0.75 \mathrm{~m}$ as a function of time together with responses for $10 \Omega$-m and $100 \Omega$-m half-space.

$22 \mathrm{~mm}$ 'shell' $8: 1$ aspect ratio at $0.75 \mathrm{~m}$ depth
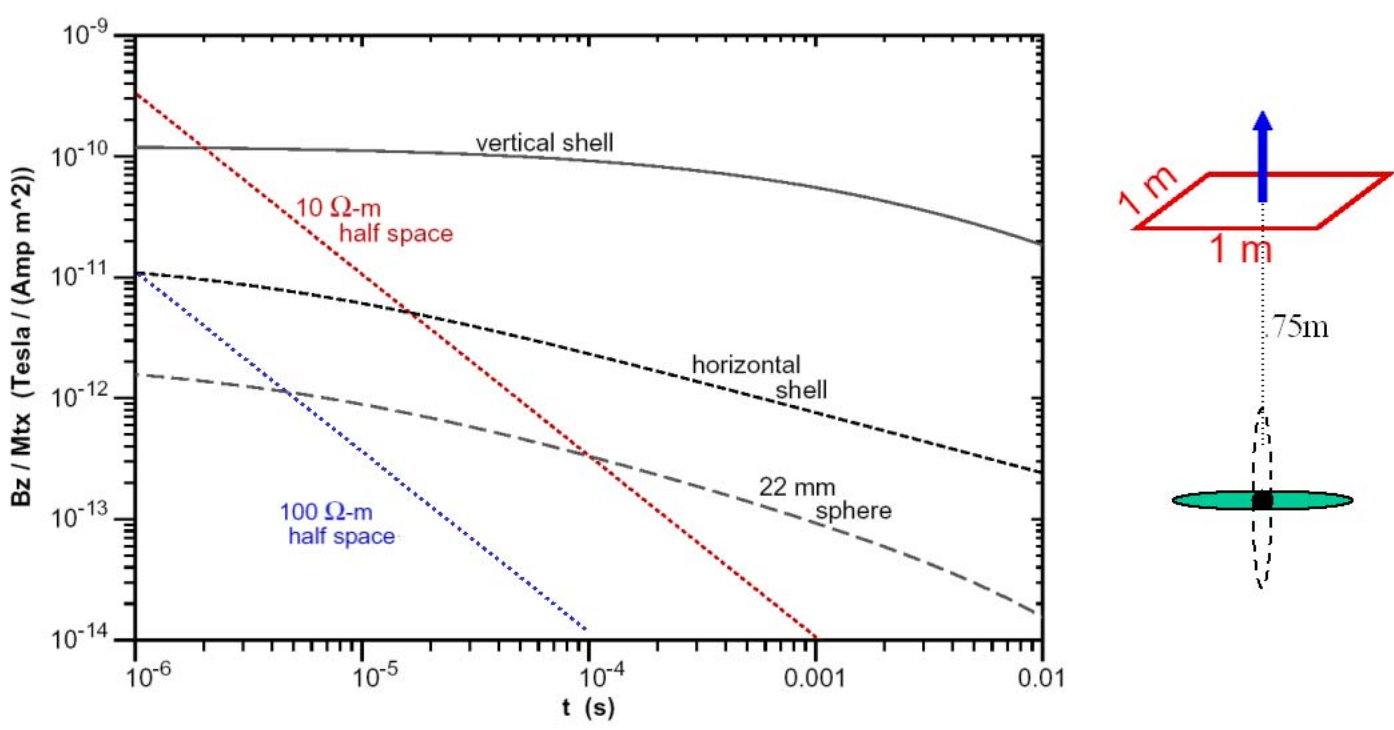

Figure 3.3.10: Amplitude of magnetic field response for $22 \mathrm{~mm}$ sphere, horizontal and vertical shells $8: 1$ aspect ratio at the depth of $0.75 \mathrm{~m}$ as a function of time together with responses for $10 \Omega$-m and $100 \Omega$-m half-space. 
$105 \mathrm{~mm}$ 'shell' $4: 1$ aspect ratio at $2.65 \mathrm{~m}$ depth

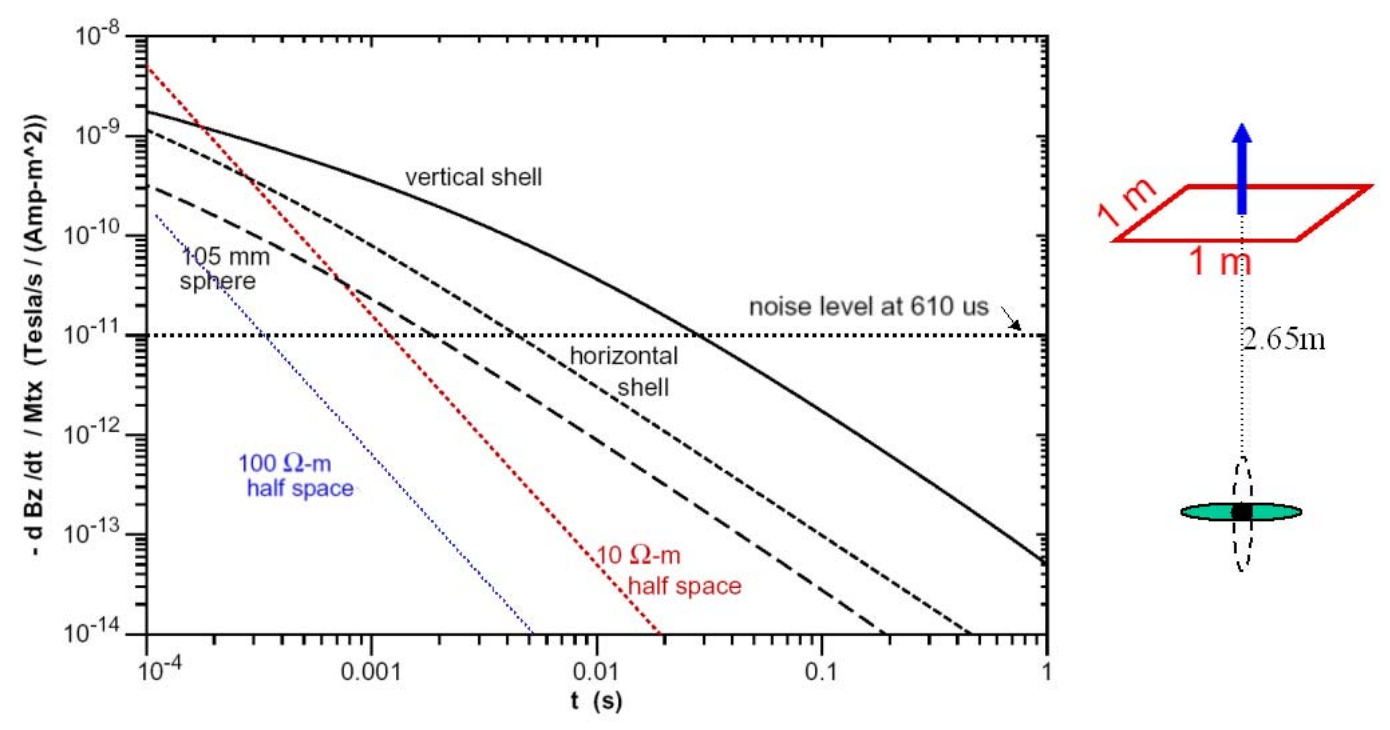

Figure 3.3.11: Amplitude of dB/dt response for $105 \mathrm{~mm}$ sphere, horizontal and vertical shells $4: 1$ aspect ratio at the depth of $2.65 \mathrm{~m}$ as a function of time together with responses for $10 \Omega$-m and $100 \Omega$-m half-space.

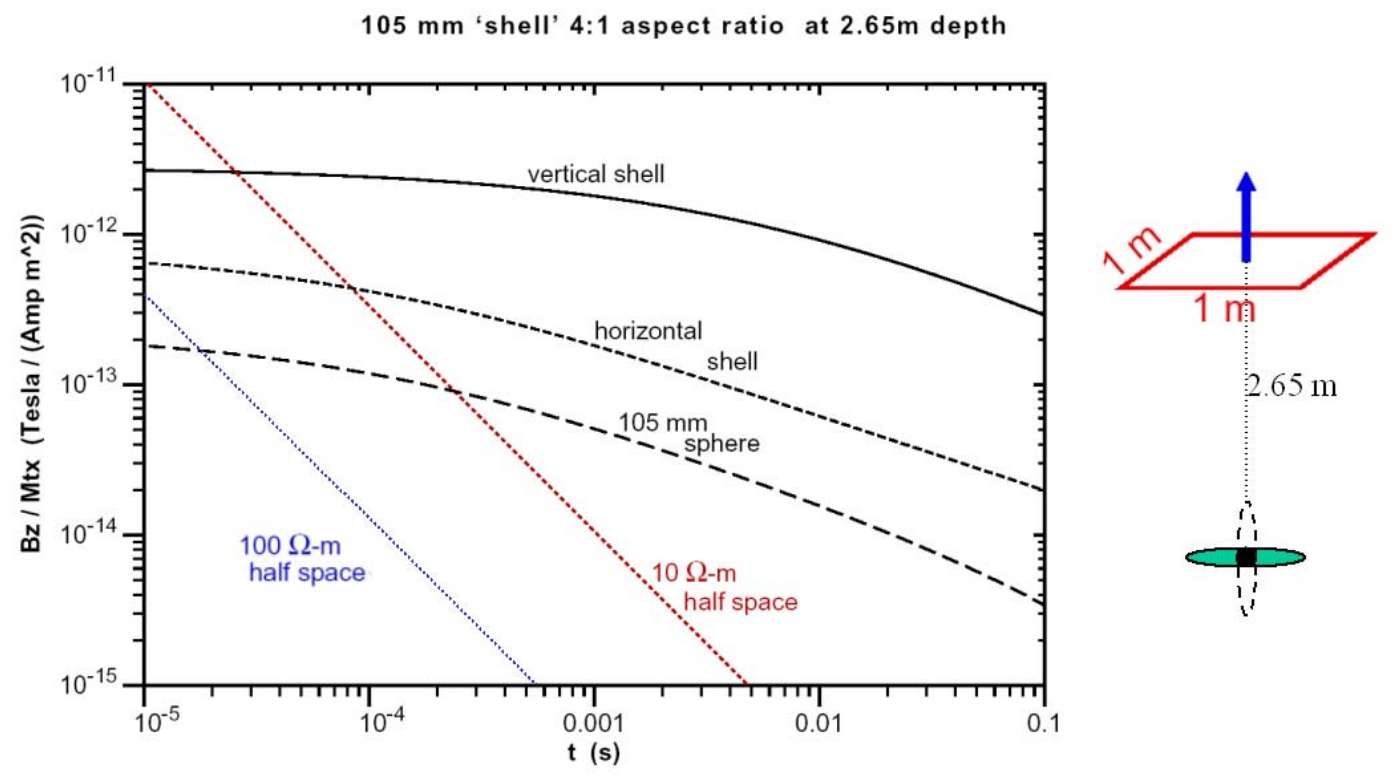

Figure 3.3.12: Amplitude of magnetic field response for $105 \mathrm{~mm}$ sphere, horizontal and vertical shells $4: 1$ aspect ratio at the depth of $2.65 \mathrm{~m}$ as a function of time together with responses for $10 \Omega$-m and $100 \Omega$-m half-space. 
$155 \mathrm{~mm}$ 'shell' $4.4: 1$ aspect ratio at $4.55 \mathrm{~m}$ depth

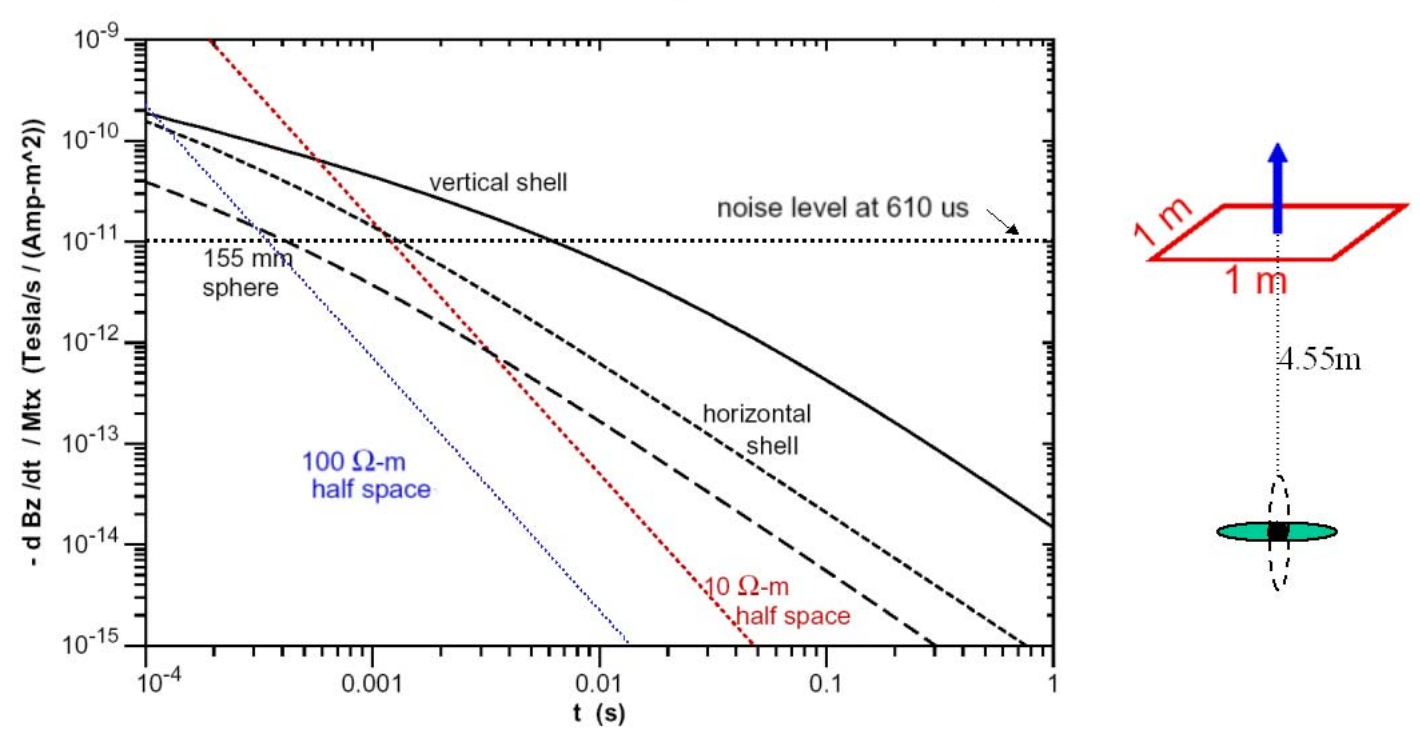

Figure 3.3.13: Amplitude of magnetic field response for $155 \mathrm{~mm}$ sphere, horizontal and vertical shells $4.4: 1$ aspect ratio at the depth of $4.55 \mathrm{~m}$ as a function of time together with responses for $10 \Omega$-m and $100 \Omega$-m half-space.

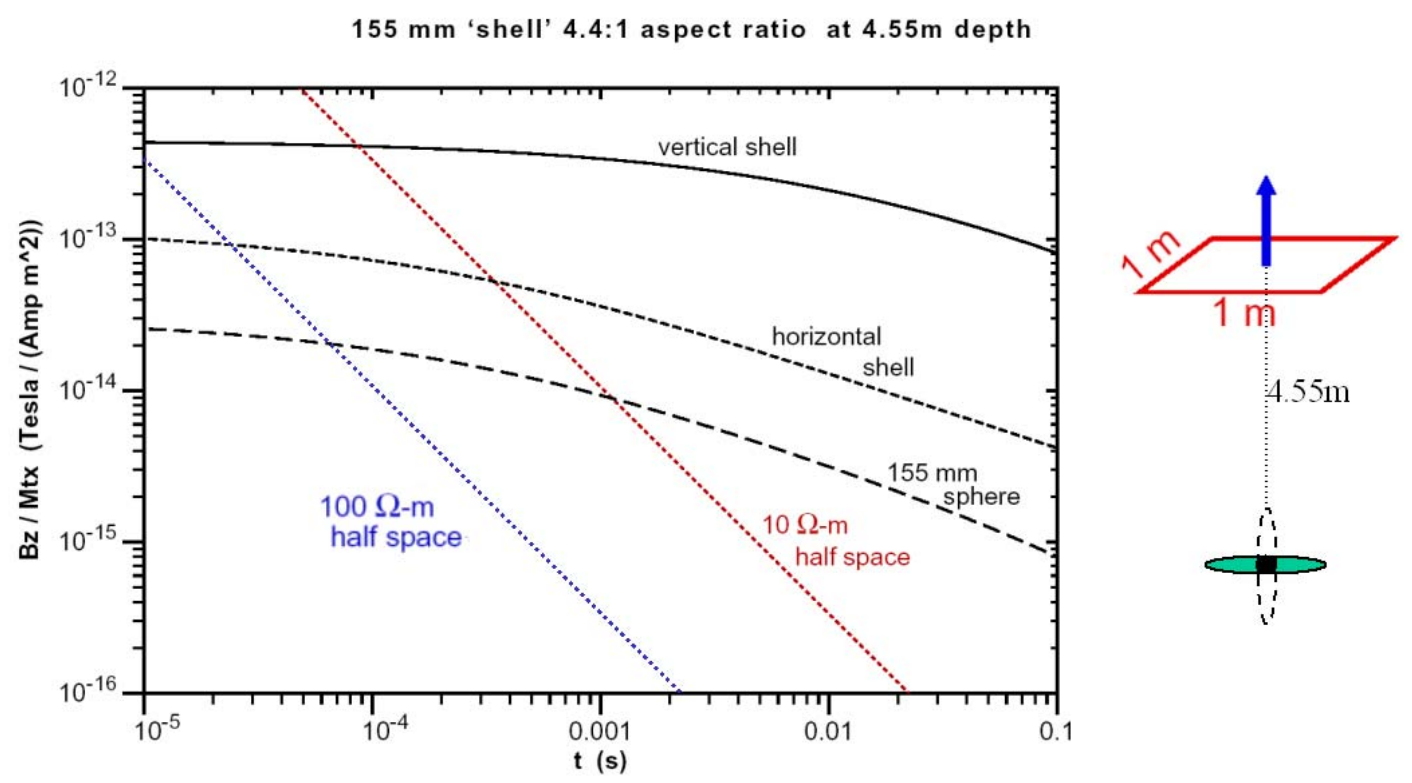

Figure 3.3.14: Amplitude of magnetic field response for $155 \mathrm{~mm}$ sphere, horizontal and vertical shells 4.4:1 aspect ratio at the depth of $4.55 \mathrm{~m}$ as a function of time together with responses for $10 \Omega$-m and $100 \Omega$-m half-space. 
These examples have been chosen to illustrate the properties of a particular system, in this case an EM61 simulation, in resolving elongate objects at a variety of depths and in showing the time window constraints imposed by the ground response and the system noise levels. The results of Section 3.2, for example Figure 3.2.5 shows the dramatic improvement in detectability that occurs when a multi-element T-R configuration is used on a grid of stations. Figure 3.2.1 for example shows that the $155 \mathrm{~mm}$ target at a depth of $4.55 \mathrm{~m}$ would not be detectable with the simple in-loop system. This of course is also seen in Figure 3.3.13 where only the vertical $155 \mathrm{~mm}$ elongated shell rises above the noise in the $6 \times 10^{-4} \mathrm{sec}$ to $7 \times 10^{-3} \mathrm{sec}$ time window. Figure 3.2.5 indicates that the three transmitter three receiver configuration, occupying many locations, could easily determine the depth and polarizabilities to better than $10 \%$ for this $155 \mathrm{~mm}$ target. A combination of detectability graphs such as Figure 3.2.5 or 3.2.11 and specific response plots such as those shown in the suite of Figures from 3.3.4 to 3.3.14 establish the bandwidth and moment requirements for an actual field system.

In summary, it appears that for step function excitation, transients from 10 microseconds to 100 milliseconds are to be detected for a practical range of UXO and a practical range of depths.

\subsection{The transient response of spheres and spheroids}

Results presented hitherto are results of an algorithm for calculating the response of a conducting, permeable sphere (including a spherical shell of arbitrary thickness) in either a uniform field or the field from a finite source. Much of our early analysis on the depth of detection, array configurations etc. was based on this spherical target. UXOs are generally not spherical and so we devoted considerable effort to adapting, and improving numerical codes to model prolate and oblate spheroidal objects. We now have reliable codes for modeling solid, conducting and permeable spheroids with aspect ratios (diameter to length) from 1:4 to 4:1. For certain polarizations of incident field the results are accurate to an aspect ratio of 10:1. 
We simulated the target response of various transmitter-receiver configurations, with step function excitation, over these spheroids for various attitudes of the bodies. For comparison we plotted the transient responses of spheres of the same radius as the equatorial radius of the spheroid and discovered that the transients were all of the same shape and were simply shifted versions of the sphere response. A typical result of this analysis is shown in Figure 3.4.1. Here we have plotted the vertical field transient response, in $\mathrm{dB} / \mathrm{dt}$ for two targets located directly below a horizontal loop transmitter at the depth of $0.75 \mathrm{~m}$. The plot shows the transient response of a $37 \mathrm{~mm}$ (equatorial diameter) prolate spheroid with an aspect ratio of 3:1. The length of the spheroid is thus $111 \mathrm{~mm}$. The response is shown for the spheroid oriented vertically and horizontally. For comparison the responses of a $37 \mathrm{~mm}$ and $111 \mathrm{~mm}$ sphere are also plotted. Finally the two sphere responses are re-plotted after each is multiplied by a constant; 0.246 for the $111 \mathrm{~mm}$ sphere and 2.71 for the $37 \mathrm{~mm}$ sphere. The shifted sphere responses lie almost exactly on the spheroidal responses over the whole transient window. The spheres and spheroids preserve their conductivities and permeabilities through this scaling.

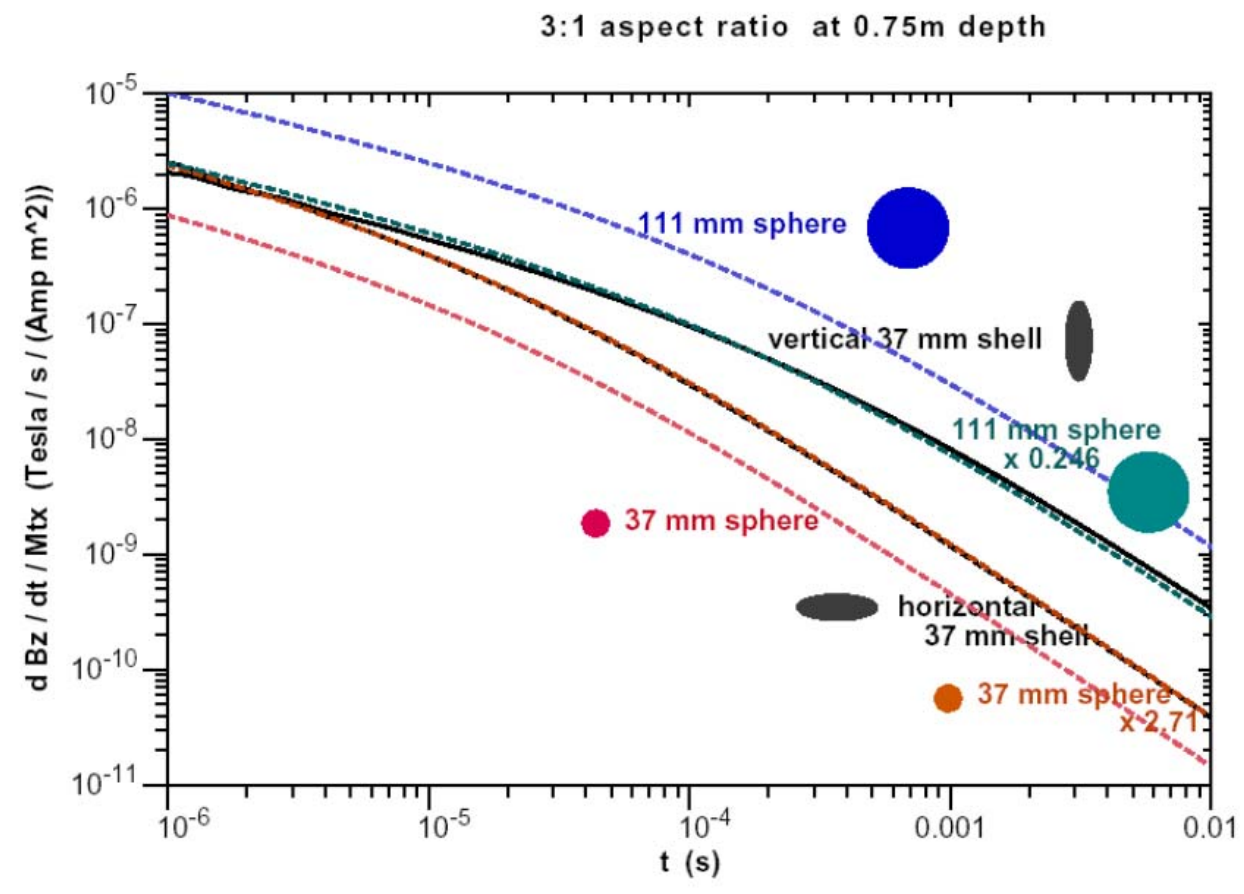

Figure 3.4.1: Equivalent sphere response. 
Smith and Morrison (2005a) have presented a detailed analysis of this empirical relationship and showed that the scaling factors are simple functions based on the volumes, dc magnetizations and high frequency limit responses of the spheres and spheroids. The scaling is valid for highly conducting bodies with relative permeabilities above 50 and with aspect ratios up to 4 or 5 to one - in other words most UXOs.

The impact of this discovery on interpreting the apparent principal polarizabilities obtained from the data is enormous. The numerical codes for obtaining the response of the ellipsoidal body are complex and are time consuming even on a large computer. Fitting an ellipsoid to the data in an inversion scheme using these numerical forward models is even more time consuming and certainly not practical for real time processing during a survey. On the other hand the algorithm for the sphere response is a closed form analytic expression, which is very fast to execute. Since the scaling factors are simple functions the dimensions of the actual spheroid are consequently derived almost instantly from the two principal apparent polarizabilities. Thus the important properties, size and true aspect ratio can be determined very quickly from the data. Finally, inversion for the conductivity and permeability can likewise be done very quickly for the equivalent spheres as described in Section 3.8.

\subsection{The effect of a repetitive transmitter waveform}

The ideal transient is modified by the practical need to use some sort of repetitive waveform rather than the unrealizable infinite step function. The usual choice is a square wave such as that shown in Figure 3.5.1. We have chosen to illustrate this analysis using B rather than $\mathrm{dB} / \mathrm{dt}$ because the results are more easily seen in the transient responses in $\mathrm{B}$ than in the more complex and rapidly decaying $\mathrm{dB} / \mathrm{dt}$ responses. The conclusions are the same in either domain. The transients in B in the off-time of the waveform are plotted for the step response and for four progressively shorter periods of the repetitive waveform. As the waveform shortens the dc component is removed and the low frequency content of the step function is cut-off. Further, there is the effect that the transient from one turn off has not recovered before it is hit by the next turn-on. Clearly the character of the transient is dramatically changed and the evident stages of the decay discussed for the step function in 
Section 3.4 above are no longer as evident. Consequently the deduction of the physical properties from the transient is compromised. From Figure 3.5.1 it is clear that the longest period possible would be best. However for optimum signal-to-noise we shall see that the waveform must be averaged, or stacked, and so the longer the waveform the longer the total time that the system must remain at a measurement point, and so the survey speed is reduced. The gain in information from lengthening the waveform is perhaps only a theoretical gain since for a practical transmitter moment the transient at late time will have fallen below the noise floor.

One way to address this problem is to simply plot the initial amplitude of the B transient for some simple transmitter-receiver configuration over spheres of various sizes and at various depths. The amplitudes for four representative spheres at four different depths are plotted as a function of repetition period in Figure 3.5.2. This range of target size and depth encompasses the range required for useful UXO detection and characterization. The amplitudes reach an asymptote with increasing period, the period at which this occurs, $\mathrm{T}_{\mathrm{c}}$, varies from about $10^{-2}$ seconds for the large sphere to $10^{-4}$ seconds for the small sphere. The transition to the asymptotic amplitude is slow - the values drop less than order of magnitude when the period shrinks by two orders of magnitude. For a practical system the period could lie between $10^{-3}$ and $10^{-2}$ seconds (repetitive rate of 1000 $\mathrm{Hz}$ and $100 \mathrm{~Hz})$.

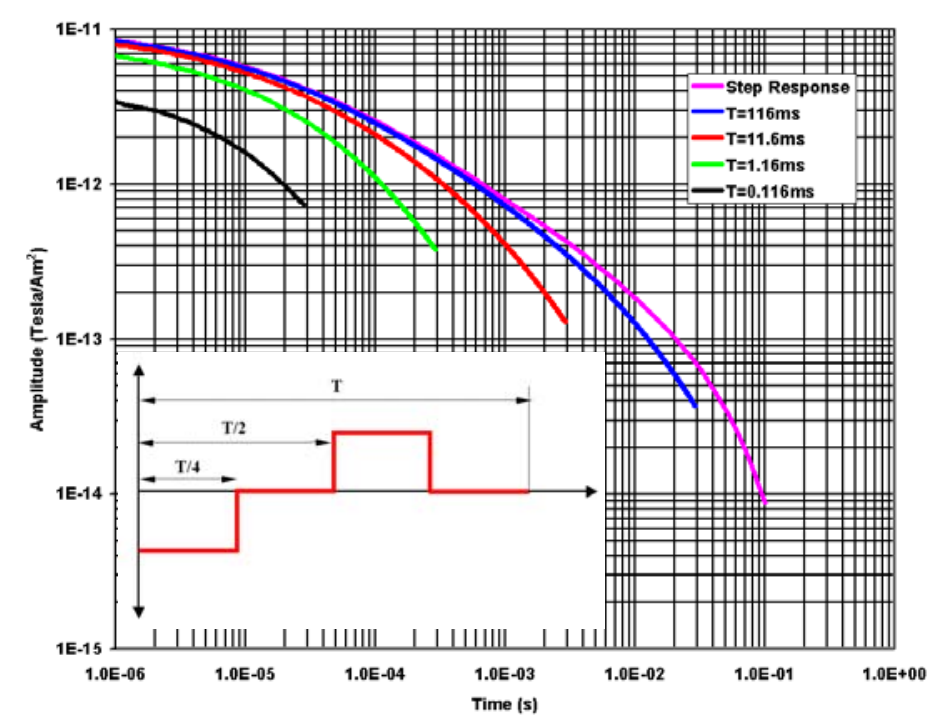

Figure 3.5.1: Transient response of a repetitive square waveform. 


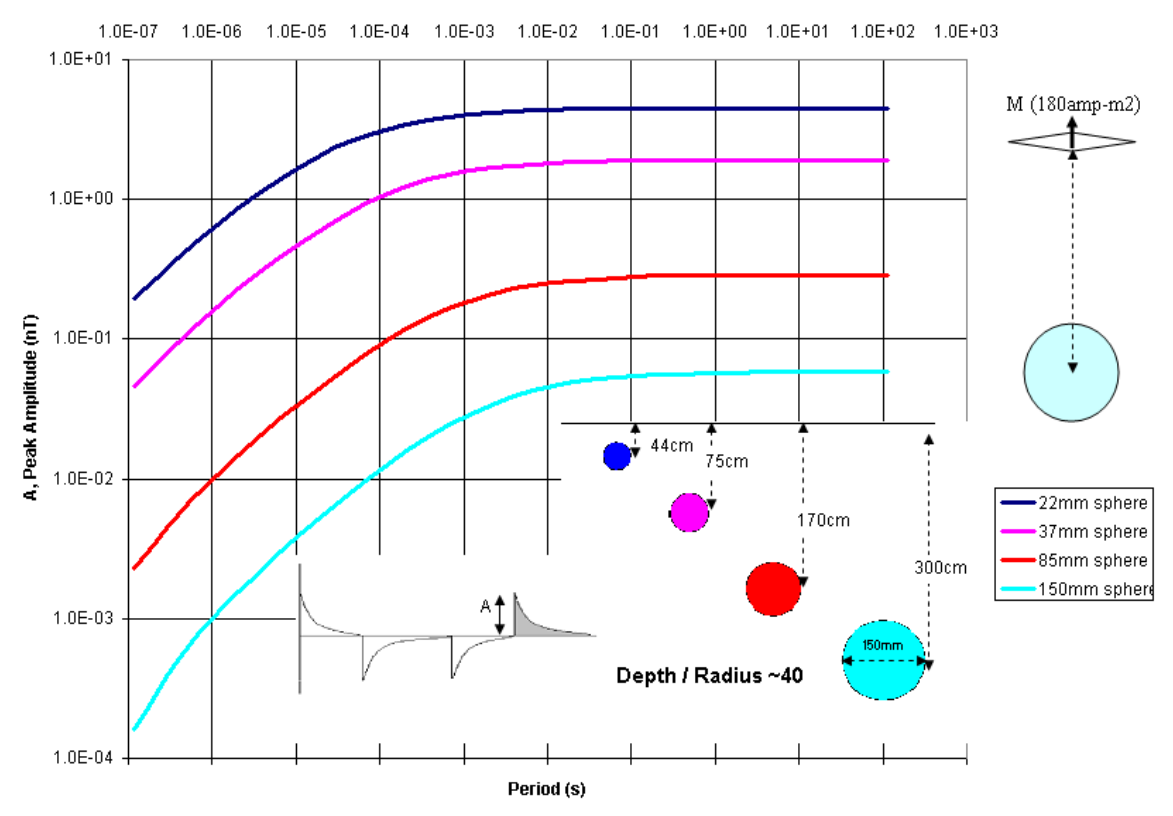

Figure 3.5.2: The amplitudes for four representative spheres at four different depths plotted as a function of repetition period.

We addressed this problem in a more formal matter by using the forward model simulator, now expanded to include the response of the band limited receiver and the square wave repetitive waveform, in an inverse mode to determine the accuracy of recovery of the physical properties for various periods of the waveform (Section 3.8). This process basically allows us to find the shortest period for which the properties can be recovered with the best accuracy.

For this analysis we used the repetitive waveform of Figure 3.5.3. The analysis included the repetition rate, $1 / \mathrm{T}$, and the relative pulse length, $\mathrm{a} / \mathrm{b}$. The current pulse amplitude was varied such that the product of the current squared and the pulse duration was kept constant - in other words the energy per pulse was the same. 


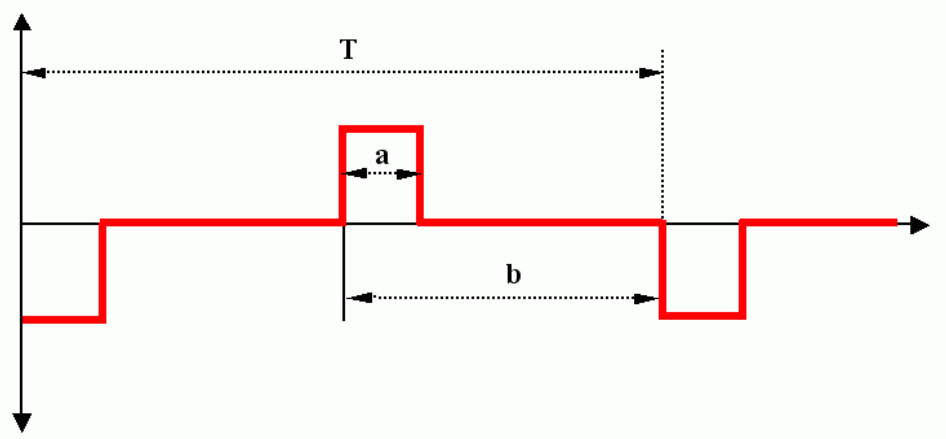

Figure 3.5.3: Repetitive waveform.

The receiver was critically damped and we used two resonant frequencies $\left(\mathrm{f}_{0}\right), 3.2$ $\mathrm{kHz}$ and $33 \mathrm{kHz}$. The target was a $160 \mathrm{~mm}$ diameter sphere. The data were inverted to determine $\log (r)$, the $\log$ of the ratio of the conductivity to relative permeability, $\log \left(\sigma / \mu_{\mathrm{r}}\right)$, and the $\log$ of the product of conductivity and permeability, $\log (\sigma \mu)$. As discussed in Section 3.6 the product $\sigma \mu$ is not well resolved from practical transient data because the transient cannot be obtained at times large enough for the exponential decay to have occurred. However for large $\sigma \mu$ even if roughly estimated, the radius, $r$, and the ratio of $\sigma / \mu$ are well determined. In other words the determination of $\sigma / \mu$ is very weakly dependent on the value of $\sigma \mu$.

The results are displayed in Figures 3.5.4 and 3.5.5 as contours of the uncertainty in the estimates of $\log (r), \log (\sigma / \mu)$, and $\log (\sigma \mu)$ as a function of the repetition rate in Hertz (on the horizontal axis) and relative pulse length (on the vertical axis). The range of repetition rates was based on rough analysis such as that in Figure 3.5.2.

Figure 3.5.4a, b, and c show results for a resonant frequency of $3.2 \mathrm{kHz}$. For both $\log (\mathrm{r})$ and $\log (\sigma / \mu)$ there is a broad 'valley' of lowest uncertainty running diagonally up to the right for both parameters. These results suggest that an optimum repetition rate is in the $10-100 \mathrm{~Hz}$ range for relative pulse lengths from 0.03 to 0.1 respectively.

The practical choice is guided by the voltage and current waveforms for the constant energy pulse. The short pulse $(\mathrm{a} / \mathrm{b}=0.03)$ would have to have a very high current with accompanying high voltages $(\mathrm{V}=\mathrm{L} * \mathrm{~d} / \mathrm{dt})$ on the switch.

The exercise is repeated for a resonant frequency of $32 \mathrm{kHz}$ and the results are plotted in Figure 3.5.5a, b, and c. Here the repetition rate range has been extended to slow 
the rapid deterioration in the estimates of $\log (\mathrm{r})$ and $\log (\sigma / \mu)$ above a few hundred Hertz. The optimum range is not dramatically different - a repetition rate of $100 \mathrm{~Hz}$ and a relative pulse length of 0.1 would still be adequate.

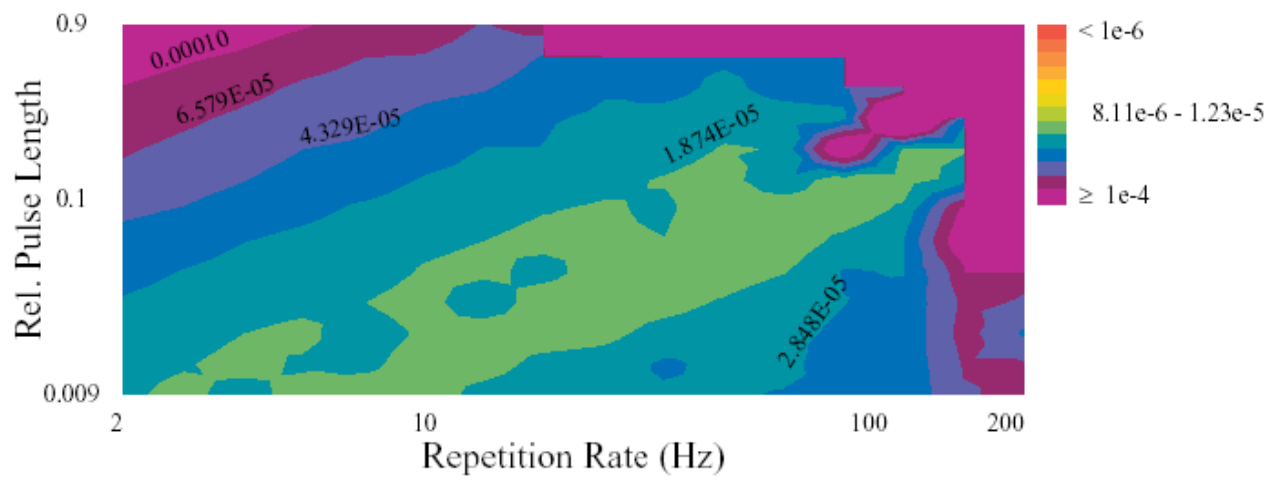

(a)

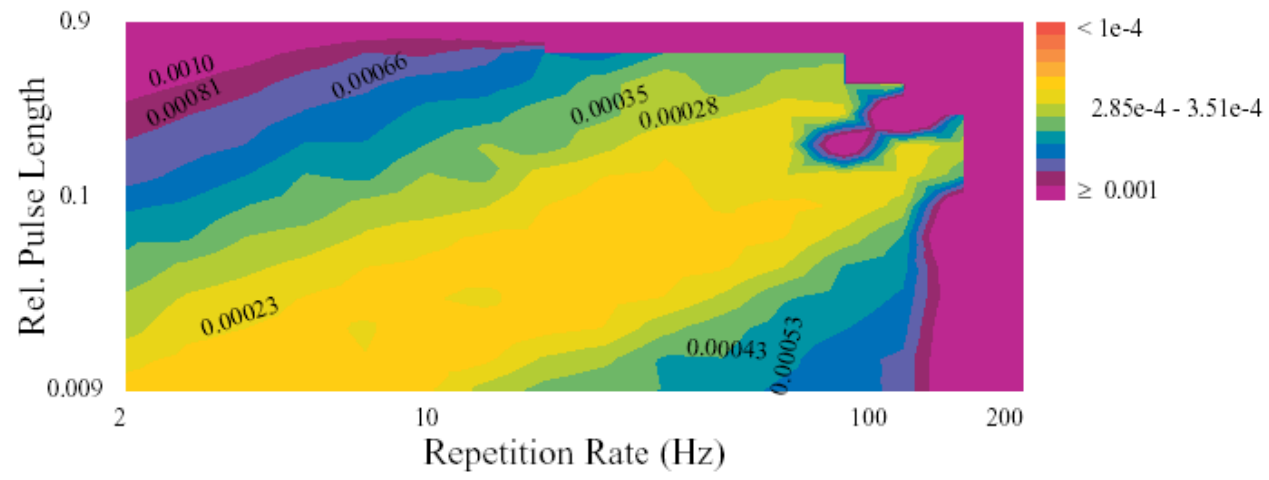

(b)

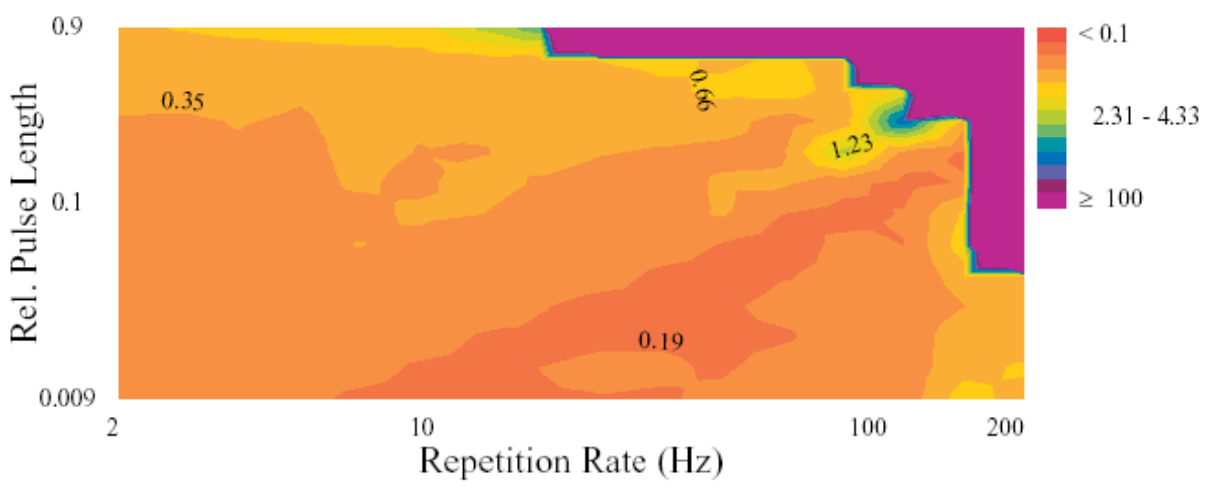

(c)

Figure 3.5.4: Uncertainties in (a) $\log (\mathrm{r})$, (b) $\log (\sigma / \mu)$, and (c) $\log (\sigma \mu)$ for $160 \mathrm{~mm}$ sphere, resonant frequency $\mathrm{f}_{0}=3.2 \mathrm{kHz}$, and critically damped receiver. 


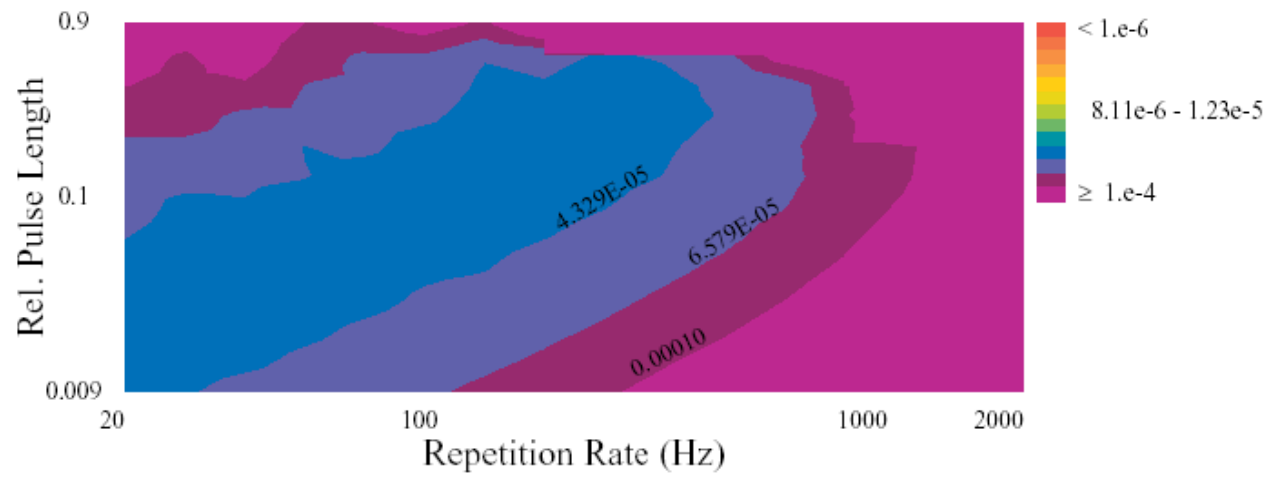

(a)

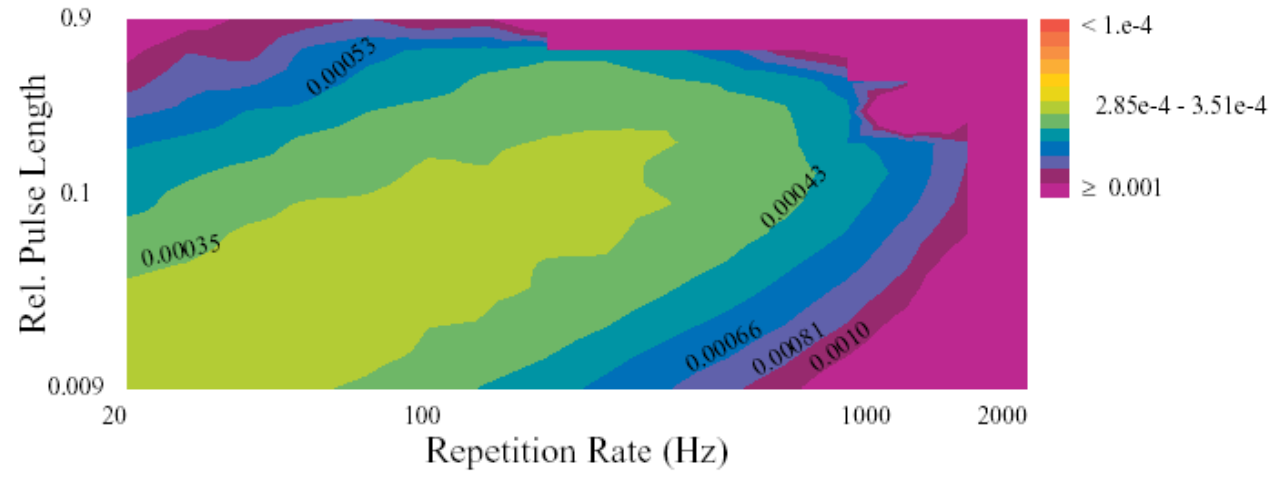

(b)

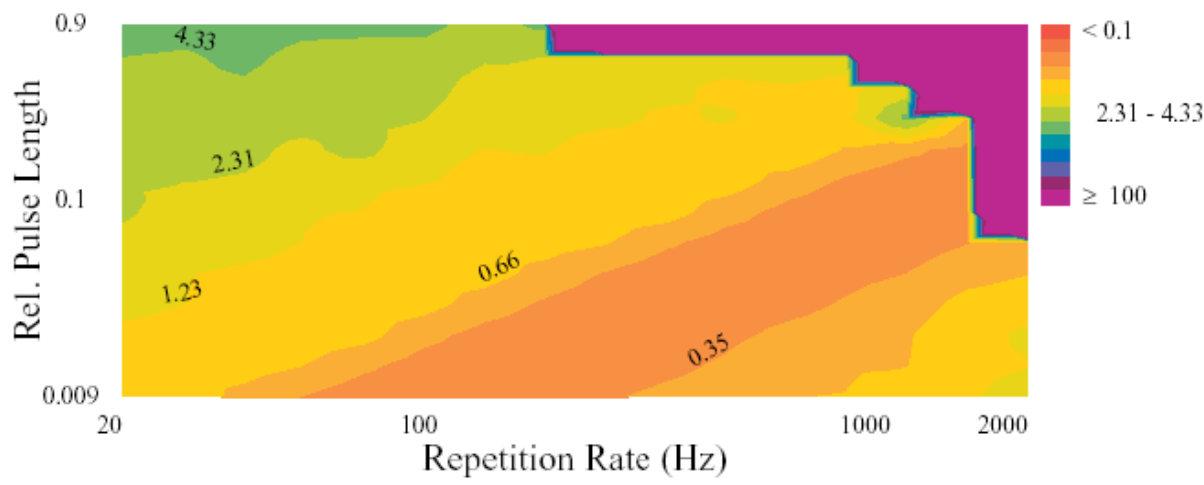

(c)

Figure 3.5.5: Uncertainties in (a) $\log (\mathrm{r})$, (b) $\log (\sigma / \mu)$, and (c) $\log (\sigma \mu)$ for $160 \mathrm{~mm}$ sphere, resonant frequency $\mathrm{f}_{0}=33 \mathrm{kHz}$, and critically damped receiver.

\subsection{Inversion for size, conductivity, and permeability}

In preliminary analysis, data from a set of receivers and transmitters is reduced to estimates of equivalent dipole polarizability as a function of time in (estimated) principal coordinates of an object, with accompanying error estimates. Given the good 
approximation of spheroid responses by appropriately scaled sphere responses, axial and transverse spheroid responses can be interpreted in terms of equivalent spheres. Dipole polarizability as a function of time is inverted for sphere radius, $r$, conductivity, $\sigma$, and relative permeability, $\mu_{\mathrm{r}}$, using the empirical distribution evolutionary algorithm of Smith et al. (1994), minimizing the weighted squared misfit between the measured dipole polarizability as a function of time and that of a sphere illuminated with a uniform incident magnetic fields with the same waveform as used for the measured data, and filtered through the same receiver transfer function as the measured data. The empirical distribution evolutionary algorithm is a population based algorithm: initially a wide variety of spheres are modeled with randomly selected parameters $r, \sigma$, and $\mu_{\mathrm{r}}$, and in a sequence of successive iterations, the space of parameters is sampled increasingly densely in the vicinity of the better points found. It is a real parameter genetic algorithm with arithmetic recombination, a high degree of tournament based elitism, with perturbations based on differences of retained population members and discarded population members. More details are given in Smith and Morrison (2004).

Once a set of sphere parameters $r, \sigma$, and $\mu_{\mathrm{r}}$ minimizing the weighted data misfit is found, parameter uncertainties are estimated using a local analysis based on the partial derivatives of the data with respect to the parameters (the Jacobian) evaluated at the minimum. Letting $\mathbf{F}$ be the Jacobian for the weighted data with respect to the logarithmic parameters $\log (\mathrm{r}), \log (\sigma)$ and $2 \log \left(\mu_{\mathrm{r}}\right)$, the covariance matrix of $\log$ parameter uncertainties is $\left(\mathbf{F}^{\mathrm{T}} \mathbf{F}\right)^{-1}$, with squared log parameter uncertainties on its diagonal and log parameter error covariances on its off-diagonals. In general, we find that estimated errors in $\log (\sigma)$ and $\log \left(\mu_{\mathrm{r}}\right)$ are highly correlated, and errors in $\log (\mathrm{r})$ essentially independent. More specifically, we have found the eigenvectors of the covariance matrix correspond very closely to $\log$ parameter combinations $\log (\mathrm{r}), \log (\sigma)-\log \left(\mu_{\mathrm{r}}\right)=\log \left(\sigma / \mu_{\mathrm{r}}\right)$, and $\log (\sigma)$ $+\log \left(\mu_{\mathrm{r}}\right)=\log \left(\sigma \mu_{\mathrm{r}}\right)$, with $\log (\mathrm{r})$ best determined, $\log \left(\sigma / \mu_{\mathrm{r}}\right)$ moderately determined and $\log \left(\sigma \mu_{\mathrm{r}}\right)$ generally very poorly determined. We find that $\log \left(\sigma \mu_{\mathrm{r}}\right)$ only becomes reasonably well determined as the length of data used in inversion approaches or exceeds the fundamental time constant of the sphere $\sigma \mu_{\mathrm{r}} \mu_{0} \mathrm{r}^{2} / \mathrm{d}^{2}$ (with $\mathrm{d} \approx 1.42 \pi$ for highly permeable spheres, $\mathrm{d} \approx \pi$ for non-magnetic spheres). 


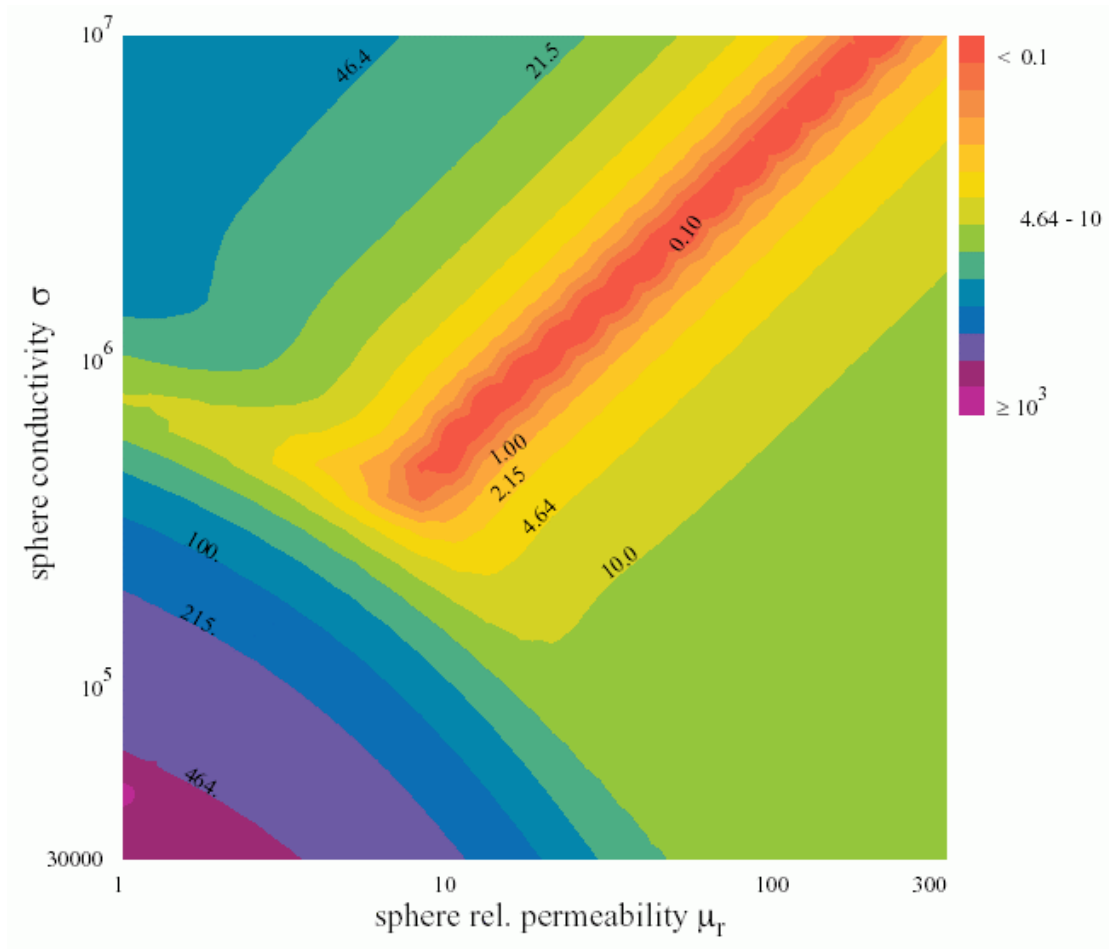

Figure 3.6.1: Squared weighted data misfit as a function of trial sphere conductivity and relative permeability for bipolar data with an $80 \mathrm{~Hz}$ repetition rate, a 0.4 duty cycle square pulse and a $32 \mathrm{k}$ radian $/ \mathrm{s}$ resonant frequency critically damped receiver using data from $0.47 \mathrm{~ms}$ to $3.75 \mathrm{~ms}$, for synthetic data from a $160 \mathrm{~mm}$ diameter steel sphere.

A graphic example of the insensitivity of data to the product $\sigma \mu_{\mathrm{r}}$ is shown in Figure 3.6.1, which shows squared weighted data misfit as a function of trial sphere conductivity and relative permeability for bipolar data taken with an $80 \mathrm{~Hz}$ repetition rate, a 0.4 duty cycle square pulse $(2.5 \mathrm{~ms}$ each half cycle), and a $32 \mathrm{k}$ radian/s resonant frequency $\left(\omega_{0}\right)$ critically damped receiver using data from when primary field transients are assumed to have sufficiently decayed at $0.47 \mathrm{~ms}\left(=15 / \omega_{0}\right)$ to the beginning of the next half cycle at $3.75 \mathrm{~ms}$, for synthetic data from a $160 \mathrm{~mm}$ diameter steel sphere, $\left(\sigma=10^{7} /\right.$ Ohm-m, $\left.\mu_{\mathrm{r}}=180\right)$. In this figure, for each point plotted trial sphere $\sigma$ and $\mu_{\mathrm{r}}$ have been specified and the sphere radius found minimizing the squared weighted misfit. The misfit surface forms a very long narrow valley through the true $\sigma$ and $\mu_{\mathrm{r}}$ values. Local analysis shows that the product $\sigma \mu_{\mathrm{r}}$ is essentially undetermined, corresponding to moving along the bottom of the valley, however, for $\sigma \mu_{\mathrm{r}}<4 \times 10^{6} / \mathrm{Ohm}$-m the valley starts to slope up steeply. This corresponds to trial spheres with time constants less than $2 \mathrm{~ms}$ starting to fit the data poorly, so although the $\sigma \mu_{\mathrm{r}}$ product is indeterminate, the data could still place a 
floor on permissible $\sigma \mu_{\mathrm{r}}$ products. Moving from the minimum at right angles to the valley corresponds to varying the ratio $\sigma / \mu_{\mathrm{r}}$, the misfit rises quickly as the $\sigma / \mu_{\mathrm{r}}$ is much better determined.

\subsection{Choice of receiver bandwidth}

The analysis of the role of pulse length and repetition period assumed a perfect receiver, that is a receiver that did not introduce additional distortion to the transient waveform. Unfortunately realizable sensors for either $\mathrm{B}$ or $\mathrm{dB} / \mathrm{dt}$ do not possess the essentially infinite bandwidth required to measure the transient without distortion. Coils inherently have a distributed capacitance so that the voltage output is only strictly proportional to $\mathrm{dB} / \mathrm{dt}$ below some $\omega_{0}$, called the resonant frequency of the coil. Such a sensor is insensitive to dc and acts as a filter on $\mathrm{dB} / \mathrm{dt}$ distorting the transient. Some high frequency limit is desirable in any sensor to reduce the broadband ambient electromagnetic field noise. For practical reasons there is no point in admitting noise of frequencies greater than the highest frequency needed to accurately represent the transient while maintaining a good signal-to-noise ratio. Some balance must be found between moving the resonant frequency to a high value to accurately represent the transient while letting in significant noise to moving it to a low value and reducing noise but significantly distorting the transient.

A variant of this induction coil sensor uses a feedback winding around the primary sensor coil. The output voltage is fed back to this auxiliary winding through a resistor in such a way as to create a field through the primary sensor which is equal and opposite to the external field being sensed. The current in the feedback winding is thus largely proportional to the sensed field rather than its derivative. These feedback sensors have been used for many years in geophysical exploration systems and are usually described as B sensors. The feedback is not ideal and the resulting sensor response is bandwidth limited. The bandwidth cannot obviously extend to dc and it would be undesirable to let in noise above those frequencies present in the desired transient. Thus the B sensor also has a bandwidth which distorts the transient. 
To illustrate this discussion we consider two possible receivers. The corresponding bandwidths are shown schematically in Figure 3.7.1. The ideal infinite bandwidth is represented by the horizontal line. The B-field coil (Figure 3.7.1a) has a center frequency, $\mathrm{f}_{0}$, of $3 \mathrm{kHz}$ (this is actually the resonant frequency of the primary sensing coil) and has flat B response from $\mathrm{f}_{0} / 100$ to $100 * \mathrm{f}_{0}$. The critically damped $\mathrm{dB} / \mathrm{dt}$ receiver (Figure 3.7.1b) has a resonant frequency of $300 \mathrm{kHz}$ and its bandwidth is from $(\sqrt{ } 2-1) f_{0}$ to $(\sqrt{2}+1) f_{0}$.

(a)

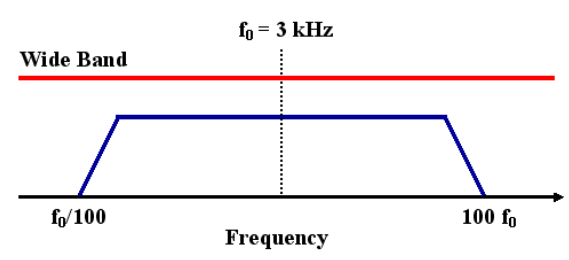

(b)

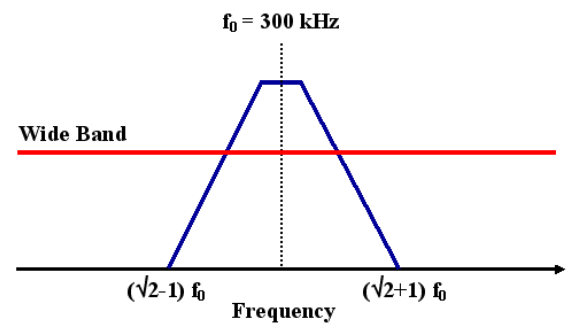

Figure 3.7.1: Schematic of the bandwidths for (a) B sensor, and (b) critically damped $\mathrm{dB} / \mathrm{dt}$ sensor.

We first note that there are two phenomena that have a major impact on the observed transient. The first is the bandwidth distortion or filtering effect described above. There is also the fact that the receiver is subject to the sharp transient turn-on and turn-off of the transmitter. The receiver, i.e. the physical coil of wire, develops an enormous emf during the transmitter transitions which persists in the coil-amplifier circuit after the cessation of the transmitter current. In a critically damped $\mathrm{dB} / \mathrm{dt}$ receiver this transient signal decays with the time constant of the coil - equal to the reciprocal of the resonant frequency of the coil, i.e. $1 / \omega_{0}$. The amplitude of this "equipment" transient is enormous so it is likely to dominate any response from a buried target for a considerable time after the turn-on or turn-off at the transmitter. This problem could be avoided by using a receiver coil oriented orthogonal to the primary field of the transmitter - the so-called minimum coupled configuration. In practice however, this is difficult to do because the orthogonality must be achieved to milliradians or less and maintained at this level as the platform is moved over the surface.

Unfortunately the problem is exacerbated in the $\mathrm{B}$, or feedback, receiver where the primary field transient is smaller but still dominates the target signal by many orders of magnitude throughout the observation interval. 
The following brief analysis of the response of a typical induction coil sensor to secondary field excitation shows in greater detail the role of the detector characteristics in the distortion of the target response.

A typical TEM system is shown in Figure 3.7.2. It consists of a transmitter with a "step-off" current waveform, an induction sensor defined by its electrical parameters L, r, $\mathrm{C}$, and a "loop" target defined by its time constant $\tau$. We will assume unity gain for the sensor amplifier so that the relationship between the sensor output and the secondary magnetic flux produced by the target is given by:

$$
V_{0}(t)=-d \phi / d t
$$

For the purpose of the following discussion we will assume that the system is null coupled so that $\phi_{0}(\mathrm{t})=0$. In the same instances it is convenient to normalize the sensor output voltage by the effective area of the sensing coil, $\mathrm{A}_{\mathrm{ef}}$. In that case, since $\phi=\mathrm{B} \cdot \mathrm{A}_{\mathrm{ef}}$ we get $\mathrm{V}_{0} / \mathrm{A}_{\mathrm{ef}}=-\mathrm{dB} / \mathrm{dt}$. Once again we see that $1 \mathrm{nV} / \mathrm{m}^{2}=1 \mathrm{nT} / \mathrm{sec}$.

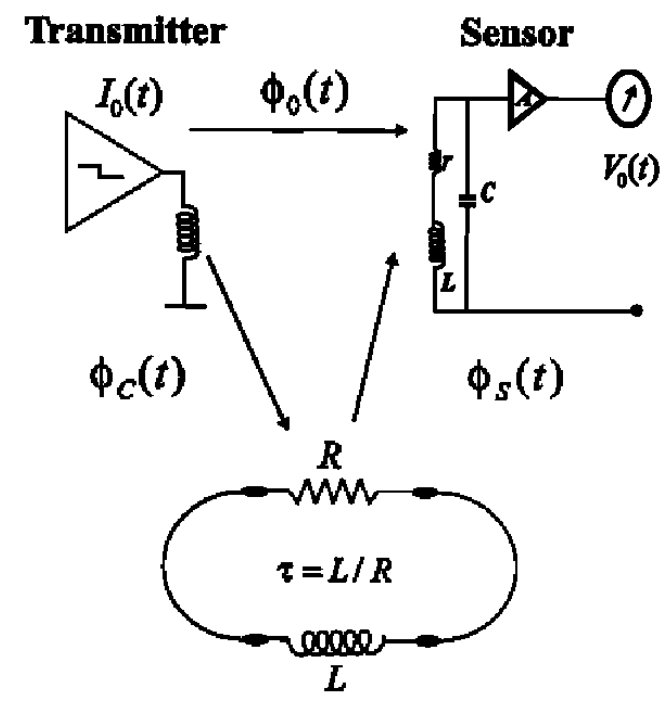

Conductor

Figure 3.7.2: A typical TEM system: It consists of a transmitter with a "step-off" current waveform, an induction sensor defined by its electrical parameters L, r, C, and a "loop" target defined by its time constant $\tau$. 


\subsubsection{The critically damped detector}

All conventional TEM systems use critically damped induction coil sensor to detect the target signal. It is either self-resonant or tuned to a central frequency $\omega_{0}$. For this type of sensor shown in Figure 3.7.2 the resonant frequency, $\mathrm{f}_{0}$, is given by:

$$
2 \pi \mathrm{f}_{0}=\omega_{0}=(\mathrm{LC})^{-1 / 2}
$$

while critical damping is assured by having

$$
\omega_{0} \mathrm{~L} / \mathrm{r}=1 / 2
$$

In that case the detector response to a sinusoidal flux signal $\phi=\phi_{0} \sin (\omega t)$ is given by:

$$
\left|\frac{\mathrm{V}}{\phi_{0} \omega_{0}}\right|=\frac{\omega / \omega_{0}}{\left(1+\omega / \omega_{0}\right)^{2}}
$$

As shown in Figure 3.7.3 the sensor bandwidth defined by the half-power points equals $2 \omega_{0}$ and spans the range:

$$
(\sqrt{2}-1) \omega_{0}<\omega<(\sqrt{2}+1) \omega_{0}
$$

In this illustration $\omega_{0}$ is set to $10^{5} \mathrm{~s}^{-1}$ corresponding to a central frequency of about $16 \mathrm{kHz}$ so that the sensor has a bandwidth of about $32 \mathrm{kHz}$.

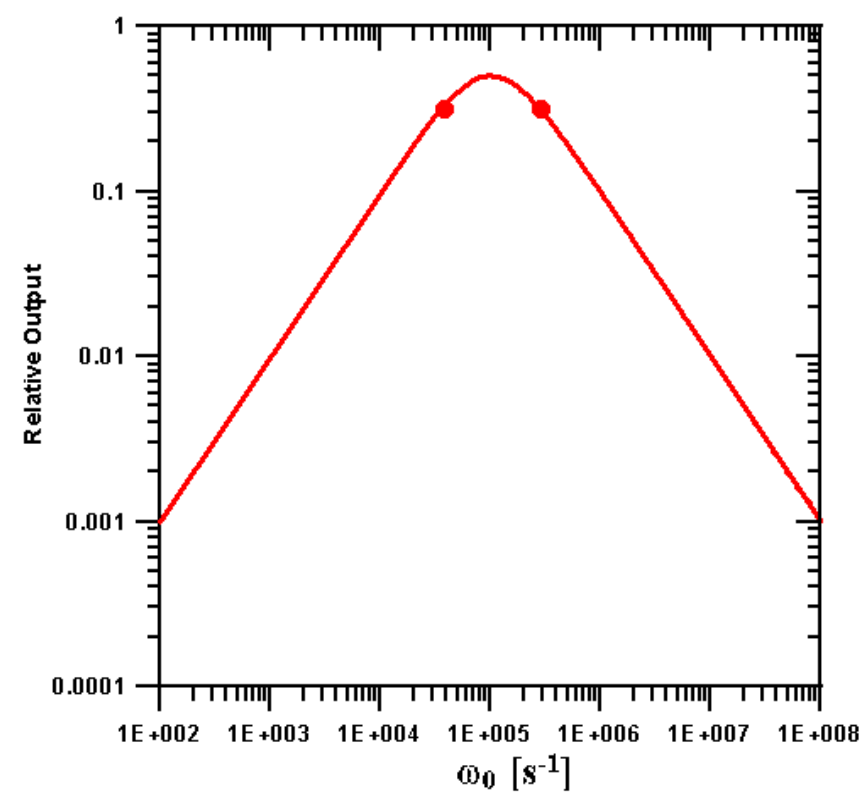

Figure 3.7.3: Bandpass characteristics for a critically damped detector from Figure 3.7.2 with a central frequency $\omega_{0}=10^{5} \mathrm{~s}^{-1}(\approx 16 \mathrm{kHz})$. 
The time domain step response, i.e. the sensor response to the primary flux for this detector is shown in Figure 3.7.4. For a unit step in the ambient flux this is given by:

$$
\mathrm{V}=\left(\phi_{0} \omega_{0}\right) \omega_{0} \mathrm{t} \mathrm{e}^{-\omega_{0} \mathrm{t}}
$$

It rises from zero to a maximum value of $10 \mu \mathrm{sec}$ corresponding to a detector rise time of 5 $\mu$ sec. In spite of this fast detector response it should be noted that for a typical maximum coupled system no observations of the secondary field can be made until the primary field "leakage" i.e. the detector response falls by about four orders of magnitude which in this case occurs at about $100 \mu \mathrm{sec}$.

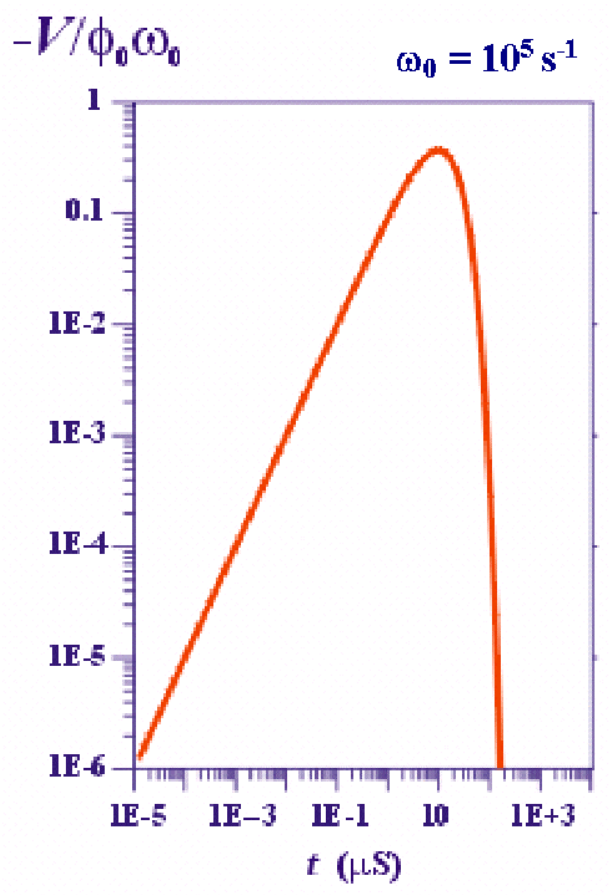

Figure 3.7.4: Sensor response to a step transition in primary flux for the induction detector from Figure 3.7.2.

Now let us turn to the effects of bandwidth on signal distortion. This is the only bandwidth related effect seen where the transmitter and receiver are in minimum coupling. Let us consider a target signal which decays with a time constant $\tau$ :

$$
\mathrm{S}_{1}=\phi_{\mathrm{S}} \mathrm{e}^{-\mathrm{t} / \tau}
$$

In this case the ideal induction detector would produce a voltage given by: 


$$
\mathrm{V}_{1} / \phi_{\mathrm{s}}=-\frac{1}{\tau} \mathrm{e}^{-\mathrm{t} / \tau}+\delta(\mathrm{t} / \tau)
$$

where $\delta(t / \tau)$ is a delta function at signal onset which is related to the leading edge of the transient signal. In normalized dimensionless form this can be written as:

$$
\mathrm{V}^{\prime}=\frac{\mathrm{V}_{1}}{\phi_{\mathrm{s}} \omega_{0}}=\frac{\mathrm{e}^{-\mathrm{t} / \tau}}{\alpha}
$$

with $\alpha=\omega_{0} \tau$. On the other hand the voltage output for a practical critically damped detector is given by:

$$
\mathrm{V}^{\prime \prime}=\frac{\mathrm{V}_{2}}{\phi_{\mathrm{s}} \omega_{0}}=-\frac{\alpha}{(\alpha-1)^{2}} \mathrm{e}^{-\mathrm{t} / \tau}+\left[\frac{\alpha}{\alpha-1} \omega_{0} \mathrm{t}+\frac{\alpha}{(\alpha-1)^{2}}\right] \mathrm{e}^{-\omega_{0} \mathrm{t}}
$$

The first term of this expression corresponds to the decaying signal somewhat amplified by a factor of $g=\left(\frac{\alpha}{\alpha-1}\right)^{2}$. The second term is the detector response to the leading edge of the signal (i.e. its initial value). It is a distorted version of the delta function part of the ideal detector response and closely resembles the actual detector step response. [The step response of an ideal detector is a delta function.]

The "ideal" and "actual" observed signals are shown in Figure 3.7.5 for a signal time constant of $100 \mu \mathrm{sec}$ and a critically damped detector resonant frequency of about 16 $\mathrm{kHz}\left(\omega_{0}=10^{5} \mathrm{~s}^{-1}\right)$. The left hand side shows the signal distortion phenomenon in true shape on linear scales. As predicted the initial part of the observed distorted signal indeed resembles a smeared out delta function while the late part of the observation is a scaled (augmented) version of the true signal. As shown in the right hand portion of the illustration the signal distortion disappears at a time of about $100 \mu \mathrm{sec}$. After this time one can extract the true time constant of the signal and, if desired, correct the observations by the appropriate "g" factor to extract the correct value of the target moment. 

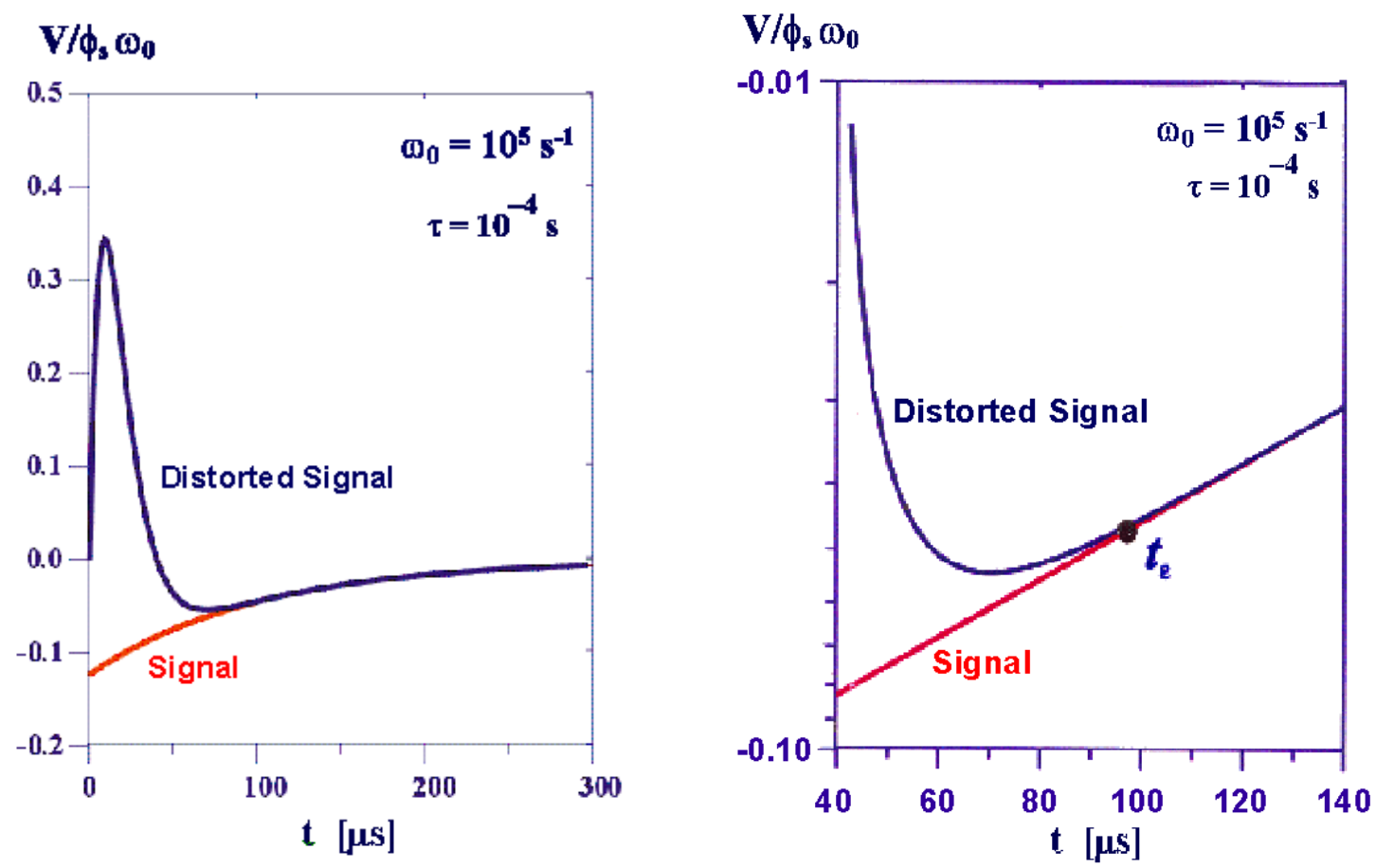

Figure 3.7.5: Distortion of the secondary field transient for a $100 \mu$ s target by a critically damped detector tuned to about $16 \mathrm{kHz}$ on linear and on log-linear scales.

We will call the instant at which the observed and augmented target signals coincide to within a given percentage the early observation time $t_{\mathrm{e}}$. It can be calculated numerically from equation (3.7.8). The results for our sample $100 \mu \mathrm{sec}$ target are shown in Figure 3.7.6. Here, for $\omega_{0}=10^{5}$, we note that we can observe the augmented transient to an accuracy of $10 \%$ at about $80 \mu \mathrm{sec}$ from the beginning of the transient while if an accuracy of $1 \%$ is needed then the earliest observation time is increased to $110 \mu \mathrm{sec}$. One can derive a general, empirical relationship between the earliest observation time for the undistorted transient and the time constant $(\mathrm{T}=\mathrm{L} / \mathrm{r})$ of the critically damped detector. For $10 \%$ accuracy and situations such as this one, where $\omega_{0} \geq \tau$, the earliest time only depends on the receiver time constant $\left(T=1 / 2 \omega_{0}\right)$ and is approximately given by $t_{e}=20 T$. Thus the earliest time at which a signal can be correctly observed is at least equal to twenty times the receiver time constant. This is so in the best of circumstances where the receiver time constant is already twenty times smaller than that of the target and we have a minimum coupled system. 


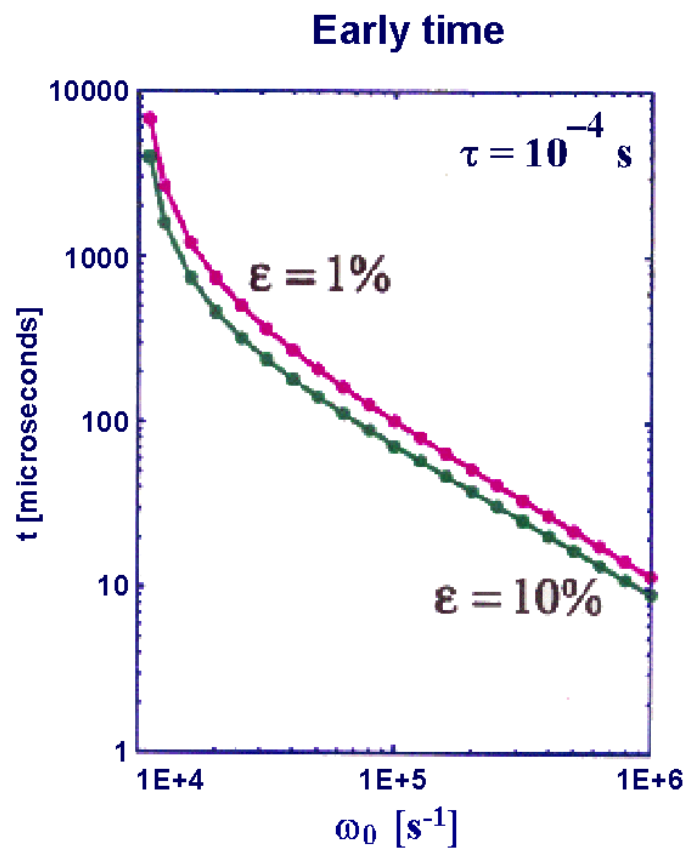

Figure 3.7.6: Earliest time of detection, to $1 \%$ or $10 \%$ accuracy for $100 \mu$ s target.

We thus conclude that at best the distortionless observation of the target signal is a difficult task.

In general the distortion introduced by the finite bandwidth of the sensor cannot be corrected by applying a system correction. The data outside the pass band is reduced below the system noise and deconvolution operations simply bring back unacceptable noise. Sensor bandwidth is reduced as much as possible, often using additional filtering in the following signal processing stages, to limit the noise but this carries the problem of distorting the transient response. Too narrow a band can distort the signal to the point where it is actually useless for detailed characterization of the target.

The feedback coil is ideally suited for optimizing the bandwidth. Depending on circumstances, the sensor can be tuned and critically damped so that it shows the conventional $\mathrm{dB} / \mathrm{dt}$ behavior. On the other hand, with the judicious use of feedback, the sensor can be made to have a flat response over many decades of frequency so that its behavior is more akin to that of magnetometer that measures B. In either case the dc target response cannot be recovered.

The idea for a sensor with wideband flat frequency response originated in France nearly forty years ago (Glerc and Gilbert, 1964). As shown below, 


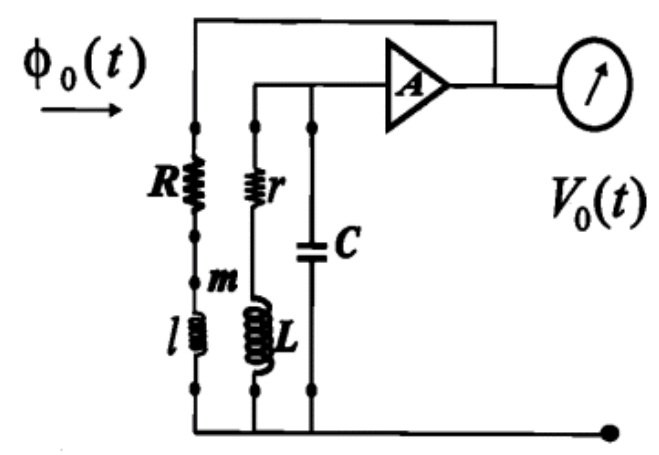

Figure 3.7.7: The wideband sensor.

This induction device consists of a principal winding denoted by its inductance " $L$ " and resistance " $r$ ". It is tuned by the capacitance " $C$ " to a central frequency " $\omega_{0}$ ". The variable bandwidth feature is incorporated by feeding back the amplifier (gain A) output through a current limiting resistor R to an auxiliary winding, "l", which is inductively coupled to the principal winding by the mutual inductance " $\mathrm{m}$ ". The receiver response is a function of the central frequency and the available feedback current. It is the latter that controls the system bandwidth as defined by the ratio of the highest to the lowest frequencies in the pass band, $\omega_{\mathrm{H}} / \omega_{\mathrm{L}}$. More precisely,

$$
\omega_{\mathrm{H}} / \omega_{\mathrm{L}}=\omega_{0} \mathrm{~m} \mathrm{~A} / 2 \mathrm{R}=\mathrm{n}^{2} .
$$

The frequency response for a variety of feedback settings as indicated by the parameter labels which correspond to values of $\omega_{\mathrm{H}} / \omega_{\mathrm{L}}$ is shown below in Figure 3.7.8.

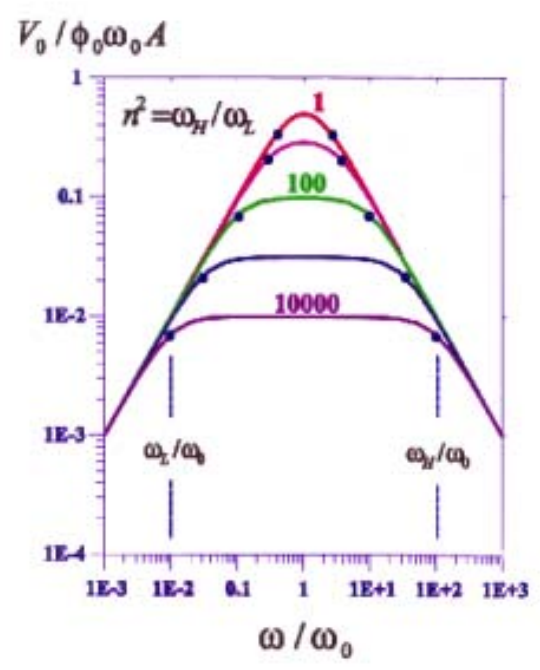

Figure 3.7.8: Frequency response of the wideband sensor. 
Here we see how the sensor response varies, with the feedback parameter " $\mathrm{n}$ ", , as a function of the normalized frequency $\omega / \omega_{0}$. A feedback parameter value of unity corresponds to the classical critically damped induction receiver with no feedback. If $\omega_{0}$, the resonant frequency of the receiver, is much greater than the frequency of peak target quadrature response then the sensor will in fact output a very close replica of the time derivative of the target signal. On the other hand, a sensor that is made to have a flat response over four decades of frequency by the application of massive feedback $\left(\mathrm{n}^{2}=\right.$ $10,000)$, will in fact have an output voltage that is closely proportional to the actual secondary magnetic field generated by the target.

The discussion to this point has considered only the goal of recovery the B response. It is considerably easier to recover the true $\mathrm{dB} / \mathrm{dt}$ response by moving the resonant frequency of the coil to a frequency much greater than the frequency of peak target quadrature response and using a feedback parameter of unity.

For a maximum coupled receiver the response is often dominated by the transient induced in the receiver by the primary filed shut-off. This phenomenon is illustrated in Figures 3.7.9 and 3.7.10. Here the sensor is in maximum coupling to a $1-\mathrm{m}^{2}$ transmitter with a moment of $180 \mathrm{Am}^{2}$. The target is a $37 \mathrm{~mm}$ iron sphere located at $75 \mathrm{~cm}$ below the transmitter plane. For the critically damped sensor the $\mathrm{dB} / \mathrm{dt}$ response of the target alone, i.e. with an ideal infinite bandwidth receiver, is denoted as 'unfiltered'. The target as seen by the critically damped receiver of Figure 3.7.1b is denoted as 'filtered'. The primary field, equipment, transient, denoted as 'primary step', is over five orders of magnitude greater than the signal transient at times earlier than $10^{-5}$ seconds. The distorting effect on the signal transient however, becomes insignificant for times greater than $25 \mu \mathrm{sec}$.

For the B sensor the primary step response is given by:

$$
\frac{\mathrm{V}}{\mathrm{A}_{\mathrm{ef}}}=\frac{\omega_{0}^{2} \mathrm{~B}}{\omega_{\mathrm{H}}-\omega_{\mathrm{L}}}\left(\mathrm{e}^{-\omega_{\mathrm{L}} \mathrm{t}}-\mathrm{e}^{-\omega_{\mathrm{H}^{\mathrm{t}}}}\right)
$$

where $\omega_{\mathrm{H}}=\mathrm{n} \omega_{0}, \omega_{\mathrm{L}}=\omega_{0} / \mathrm{n}$, with $\mathrm{n}$ defined in equation (3.7.9), $\mathrm{V}$ is the measured signal, and $\mathrm{A}_{\mathrm{ef}}$ is the effective area of the sensing coil. 
Even though the signal is now proportional to B over a large part of the frequency range it still has to be measured in Volt $/ \mathrm{m}^{2}$ or Tesla/sec. As shown in Figure 3.7.10 for the B sensor, with the bandwidth shown in Figure 3.7.1a, the primary step transient is about four decades of magnitude greater than the signal transient and it has essentially no decay up to $10^{-3}$ seconds. Any practical sensor would lack the dynamic range to separate these two phenomena.

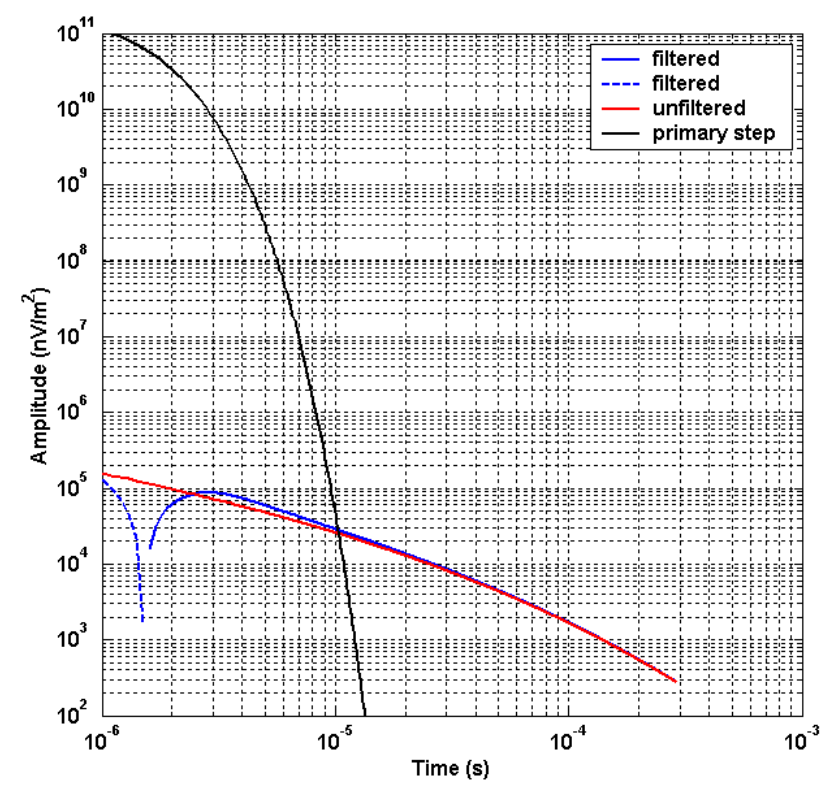

Figure 3.7.9: Critically damped $\mathrm{dB} / \mathrm{dt}$ sensor response.

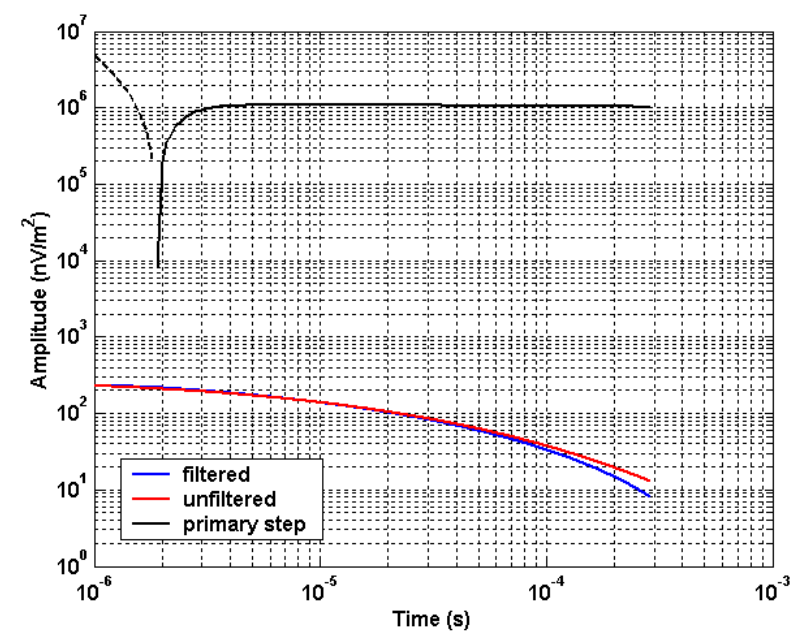

Figure 3.7.10: Wideband B sensor response. 
As might be expected the signal-to-noise ratio for any receiver is a function of its resonant frequency and bandwidth. We can see this by again taking the situation illustrated in Figure 3.7.9 and examining the effect of the resonant frequency for the critically damped receiver on the signal-to-noise ratio (SNR) and the required dynamic range. For the critically damped $300 \mathrm{kHz}$ receiver in question and ignoring system noise we find an SNR of about 25. This estimate is based on a signal observation at about $12 \mu \mathrm{sec}$ after the extinction of the primary field where the target signal has amplitude of about $25 \mu \mathrm{V} / \mathrm{m}^{2}$. For this bandwidth the natural observable noise is about $1 \mu \mathrm{V} / \mathrm{m}^{2}$.

Lowering the receiver resonant frequency to $30 \mathrm{kHz}$ would indeed reduce the noise by a factor of five to about $200 \mathrm{nV} / \mathrm{m}^{2}$ but then the target signal would not be seen until 130 $\mu$ sec after extinction where its amplitude is only $900 \mathrm{nV} / \mathrm{m}^{2}$. In this case lowering the receiver resonant frequency by a factor of ten would result in an SNR reduction by a factor of about five.

This is only an illustrative example done for external noise measured at a relatively quiet site. In a presence of large noise it is quite likely that the $30 \mathrm{kHz}$ receiver frequency would be optimal. In either case the required dynamic range would be about $160 \mathrm{db}$. The dynamic range requirement can be much relaxed by using a half sine transmitter with a much higher moment. 


\section{LAB PROTOTYPE}

\subsection{Introduction}

The analyses developed in this project are applicable to AEM systems on any scale. We have found in this process that systems scale roughly with depth of burial and size of the target. Thus the dimensions of the transmitter control the field strength at depth and spacing of receivers controls the accuracy of depth estimates. On the other hand the detection of small near surface objects depends on having enough receivers deployed spatially so as not to miss their spatially small response pattern. It appears that given the possibility of an arbitrary number of receivers that the overall system dimensions should be set by the size of the transmitter loop and the practicality of moving this loop over the ground.

We have decided in this preliminary design to choose the popular cart mounted configuration. With a horizontal loop dimension on the order of 1.0 square meter this configuration has been shown to be maneuverable and to be capable of exciting responses from typical UXO to depths of a meter or two. In a sense we have chosen a conservative design based on systems which have a demonstrated track record of detecting metallic objects to the depths of interest. The important advances of the proposed design are that the data can be used to characterize the target and in so doing classify it as possible UXO or as scrap, and that the target can be located and identified from a single position of the multi-sensor system. The following sections summarize the considerations included in the design process.

\subsection{Prototype system}

We assembled a bench prototype system illustrated schematically in Figure 4.2.1. This system was not rigorously optimized along the lines suggested in Section 3, rather it was assembled with readily available components to get a working system whose performance could be used to confirm modeling results and to be a starting point for an optimized system. 


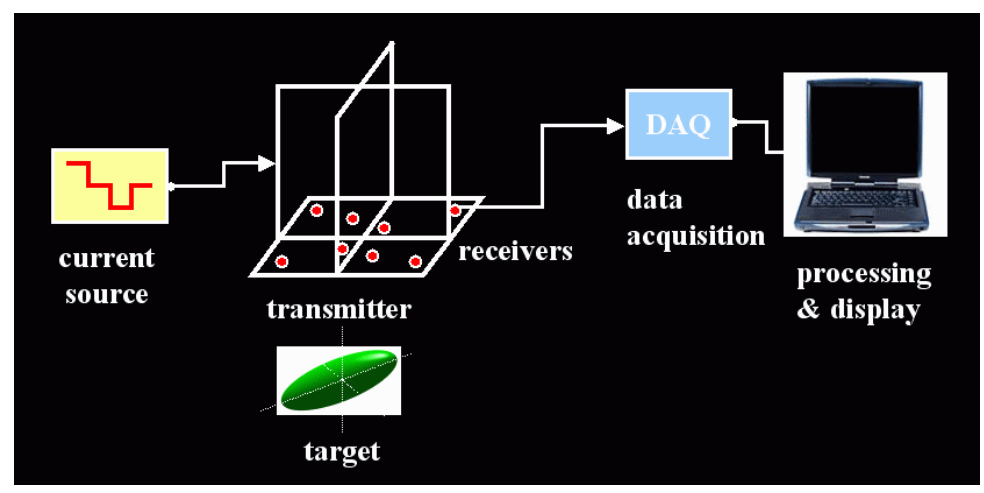

Figure 4.2.1: Schematics of the bench test acquisition setup.

The main transmitter loop is the one used in the Geonics EM-61 system. It is roughly one meter square, has approximately 32 turns. We used a half sine of 19 amperes peak for a moment of about 600. We synthesized results from an orthogonal three-coil system by simply moving the one coil to three positions in the jig shown in Figure 4.2.2.

We used eight "pancake" receiver coils mounted within the footprint of the horizontal transmitter loop. The receivers were originally designed for an EM scale model study and are not designed for this system. Nonetheless they are adequate for this proof of concept. In Figure 4.2.2 these receivers are the round wheel-like shapes. The black centers are tapered plastic plugs, which, when screwed down on precisely located threaded posts on the horizontal platform assure that the coils are accurately relocated if they need to be removed. The choice of location of the eight receivers was made following the optimization procedure of Section 3.2.

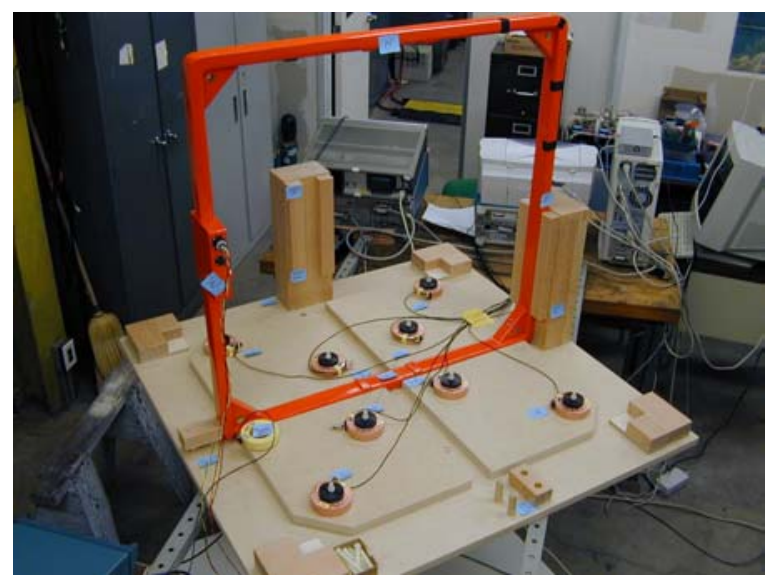

Figure 4.2.2: Photo of the prototype system. 
The current source is a repetitive bipolar half sine waveform the parameters of which are shown in Figure 4.2.3. Again we used a half sine pulser developed in a previous project. Based on the discussion of Section 3.5 the repetition rate is good but the relative pulse length is probably too large.

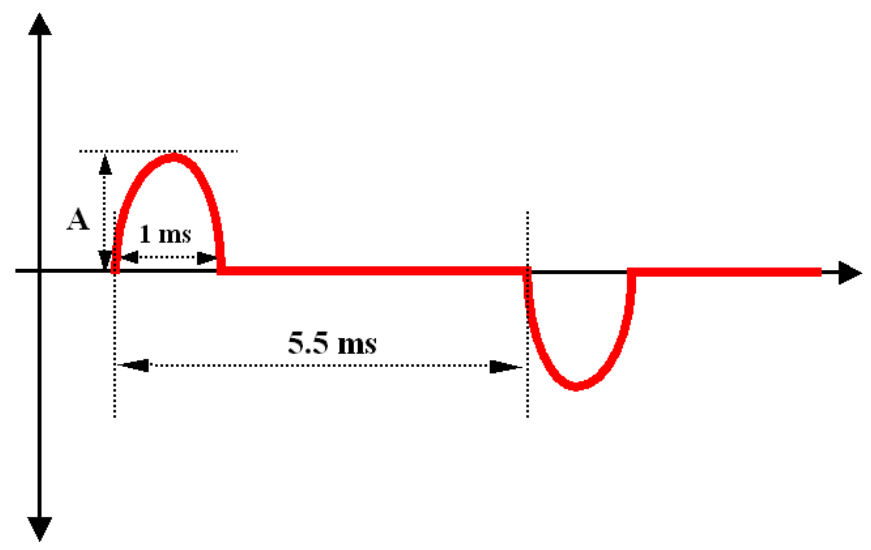

Figure 4.2.3: Half sine waveform of prototype system - repetition rate of $90 \mathrm{~Hz}$, relative pulse length is 0.18 , and maximum current $\mathrm{A}=19$ Amperes.

Each receiver in this prototype is connected to a channel of a multi-channel oscilloscope by a simple twisted pair cable. The trigger for the oscilloscope is yet another receiver coil mounted essentially on the transmitter winding. The scope is thus triggered on the trailing sharp edge of the half sine derivative. Recording is delayed by $20 \mu \mathrm{sec}$ to avoid switching transients and the equipment transients discussed in Section 3.4.

The transient is digitized with a sampling interval of $2 \mu \mathrm{sec}$. The digitized transient is averaged, or stacked, over 100 records and the averaged transient is sent to a computer for storage and processing.

The system has a response from all metallic objects within a radius of two or three dimensions of the object. Unfortunately, this means that there will be spurious response from the laboratory equipment, nearby filing cabinets or rebar in the floor etc. We decided to deal with this problem by recording a set of averaged transients with no test target present and then subtracting this transient from that obtained with the target present. All the transients obtained in the following test were treated in this manner.

The transmitter-receiver system was mounted about one meter off the floor and various target objects were placed beneath it. The individual points of this transient were 
further averaged into time windows whose width is equal to $25 \%$ of the center of the center frequency of the window. This reduced the number of points representing the transient to about 30. The averaged transients for each receiver, and for three orthogonal positions of the transmitter were then used to invert for the location and principal polarizabilities (and the orientation, azimuth, and dip of the major polarizability).

The inversion stopped at this stage. With the polarizabilities determined as a function of time it is possible to utilize the results of Sections 3.3 and 3.6 to determine the physical aspect ratio, $\log (\mathrm{r})$ and $\log (\sigma / \mu)$. These results were for a repetitive square wave and algorithms have not yet been modified for the half sine waveform used in the prototype.

\subsection{Test model results}

We used three simple targets in the initial tests; a simple multi-turn coil $18 \mathrm{~cm}$ in diameter, a copper sphere $138 \mathrm{~mm}$ in diameter and a steel ellipsoid (Figure 4.3.1) 9 inches long and 3 inches in diameter (aspect ratio of 3:1). For each target the location (x, y, z), the three principal moments and the orientation of the three principal axes are plotted as a function of time on the transient. The estimates are plotted as solid, colored, lines and dashed lines of the same color indicate the variability of the estimate. On each plot the actual value of the parameters plotted is listed.

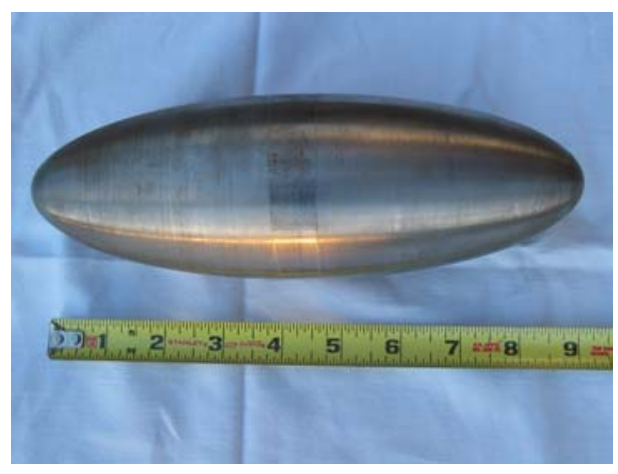

Figure 4.3.1: The 9 inch $(23 \mathrm{~cm})$ long and 3 inches $(7.6 \mathrm{~cm})$ in diameter steel ellipsoid.

Figures 4.3.2a, b, c are plots of the location, principal moments and principal axes directions (sometimes given as direction cosines) for the $18 \mathrm{~cm}$ diameter shorted multi-turn loop. The loop was positioned about $30 \mathrm{~cm}$ below, and coaxial with, the horizontal 
transmitter loop. The location, Figure 4.3.2a, is recovered very accurately between $5 \times 10^{-5}$ sec and $5 \times 10^{-4}$ sec. The principal moment, Figure $4.3 .2 \mathrm{~b}$, there should be only one for a planar loop, is well recovered over the same time interval, and the minor moments, which should be zero, are about two orders of magnitude less than the principal moment - they are in the noise of the estimate. Finally, the direction cosines, Figure 4.3.2c, are well recovered.

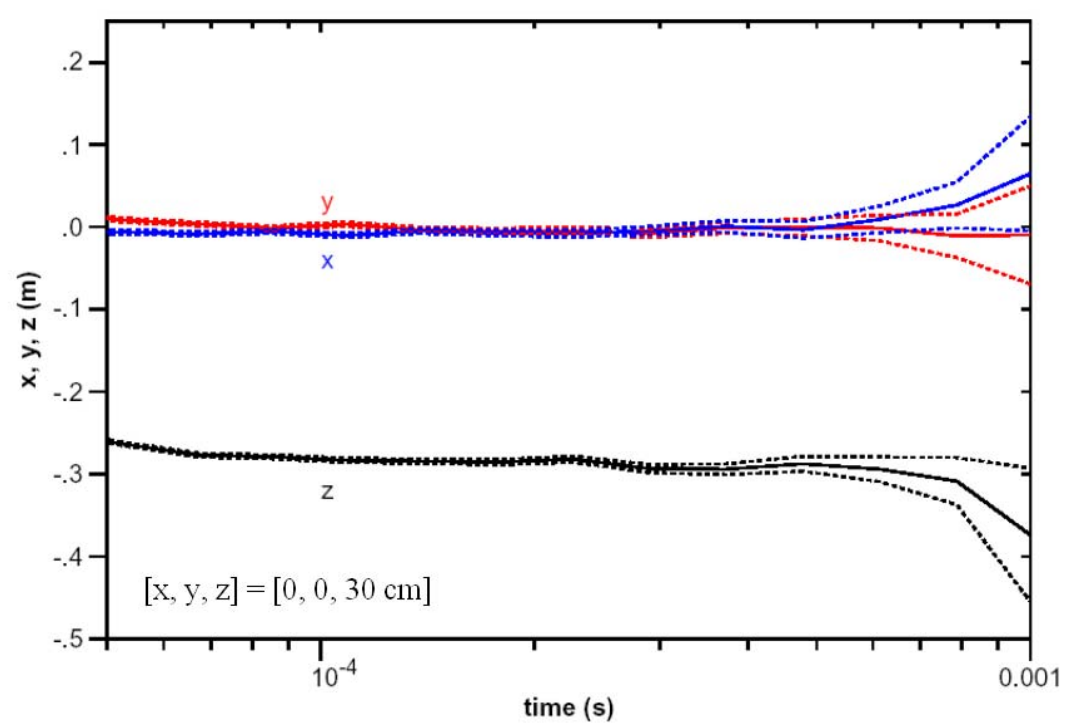

Figure 4.3.2a: Inversion results for the position of $18 \mathrm{~cm}$ diameter shorted multi-turn loop.

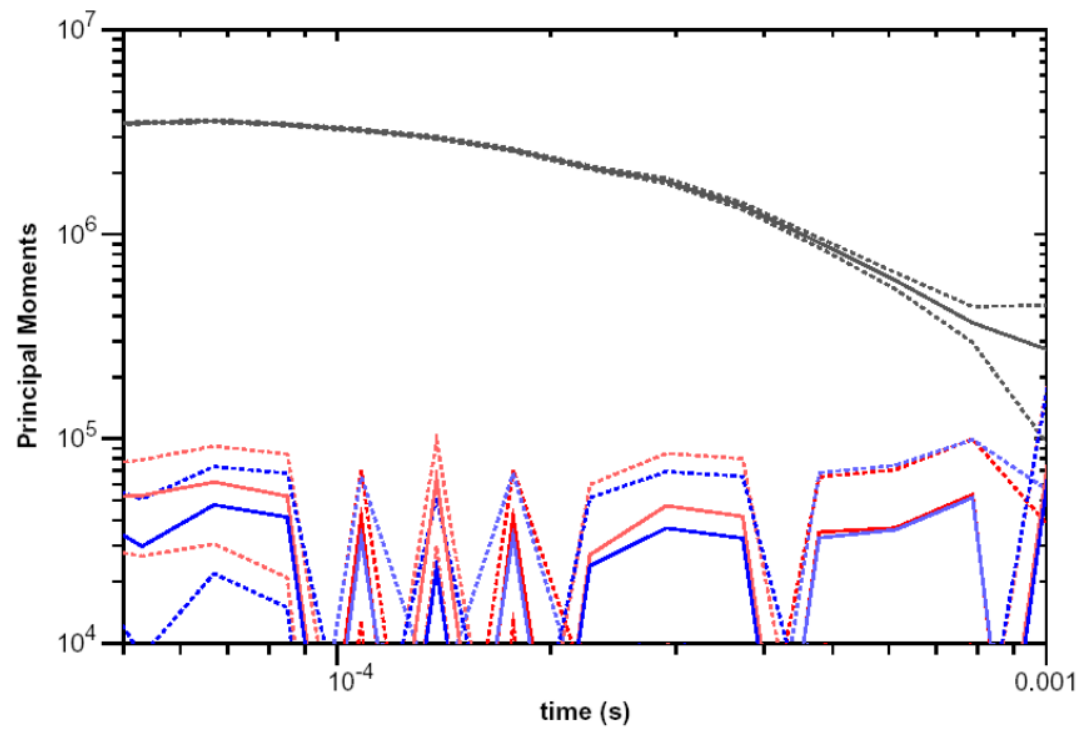

Figure 4.3.2b: Inversion results for the principal moments of $18 \mathrm{~cm}$ diameter shorted multi-turn loop. 


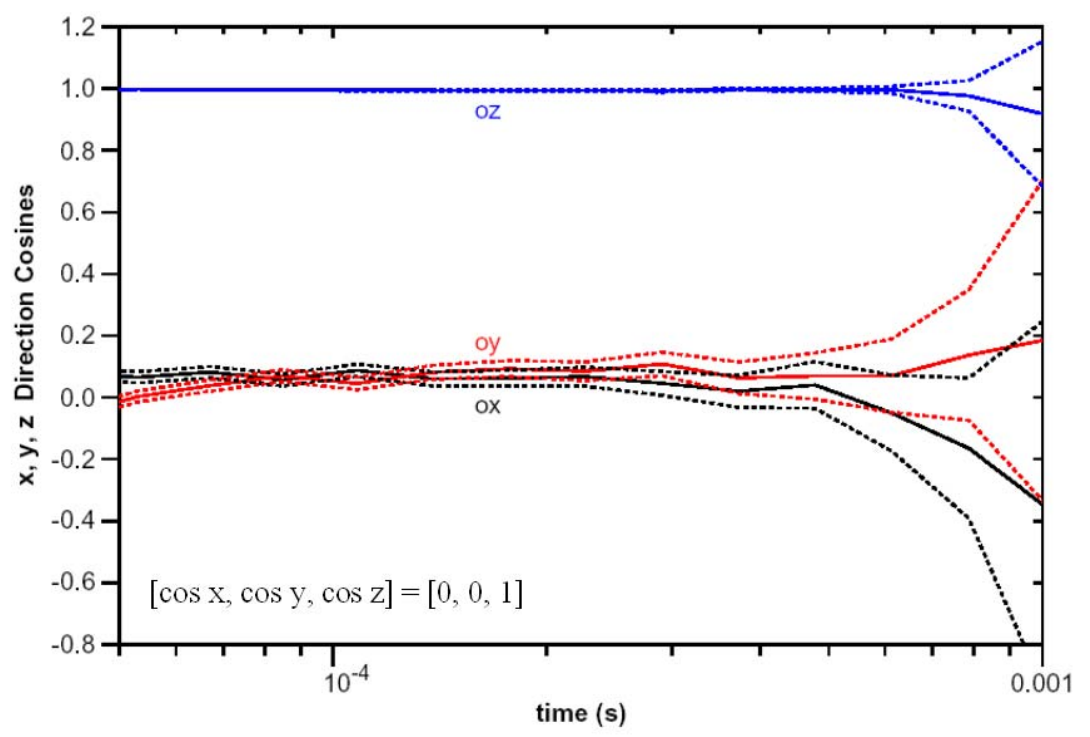

Figure 4.3.2c: Inversion results for the moment orientations of $18 \mathrm{~cm}$ diameter shorted multi-turn loop.

The results for the $138 \mathrm{~mm}$ diameter copper sphere are plotted in Figures 4.3.3a and 4.3.3b. The sphere center in this case is only $20 \mathrm{~cm}(200 \mathrm{~mm})$ below the plane of the horizontal coil so it might be wondered whether the dipole representation is accurate. Indeed the inverted parameters at 'early' time, from $5 \times 10^{-5}$ to $10^{-4}$ seconds, seem to depart in a well defined way from the true values. Nonetheless the location is well recovered (Figure 4.3.3a), and the principal dipole moments, which should all be equal, fall roughly within each others error bounds between $5 \times 10^{-4}$ and $10^{-4}$ seconds (Figure 4.3.3b). The moment orientations are meaningless in this case. 


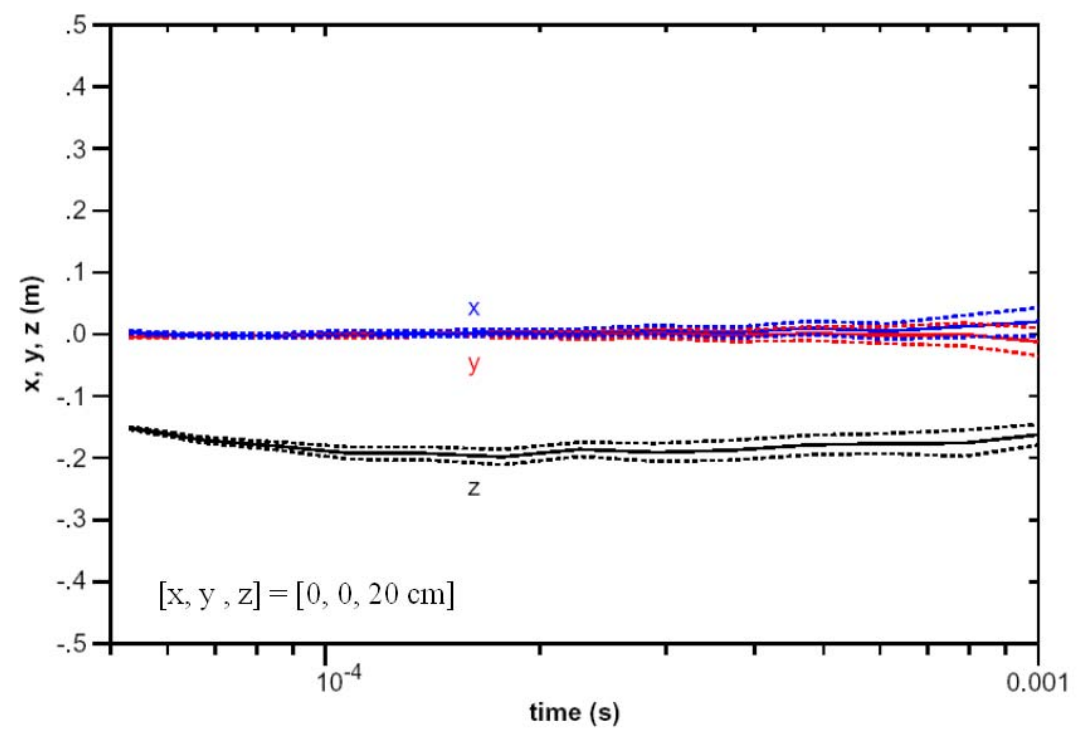

Figure 4.3.3a: Inversion results for the position of $138 \mathrm{~mm}$ diameter copper sphere.

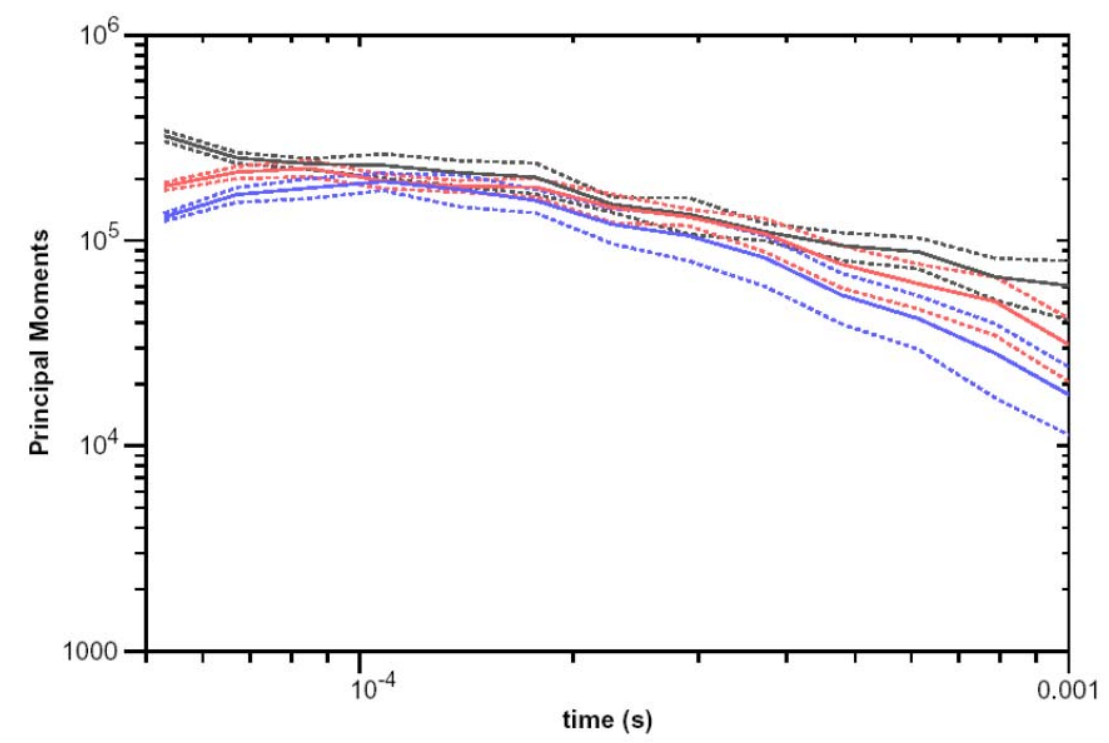

Figure 4.3.3b: Inversion results for the principal moments of $138 \mathrm{~mm}$ diameter copper sphere.

Finally, we positioned the 9 inch $(23 \mathrm{~cm})$ steel ellipsoid first directly below the center of the transmitter at the depth of $30 \mathrm{~cm}$, with its long axis horizontal $\left(\operatorname{dip}=0^{\circ}\right)$ and at an angle of $45^{\circ}$ to the principal coordinates of the system (azimuth). This test was done in an all-wood building at our Richmond Field Station and in general the noise was less. The location, Figure 4.3.4a, is recovered very well over the entire transient window, the 
principal polarizabilities are equally well resolved, Figure $4.3 .4 \mathrm{~b}$. Finally the dip and azimuth are recovered very well, Figure $4.3 .4 \mathrm{c}$, - so well in fact that it appears that ellipsoid was in fact $2^{\circ}$ off its intended $45^{\circ}$ azimuth.

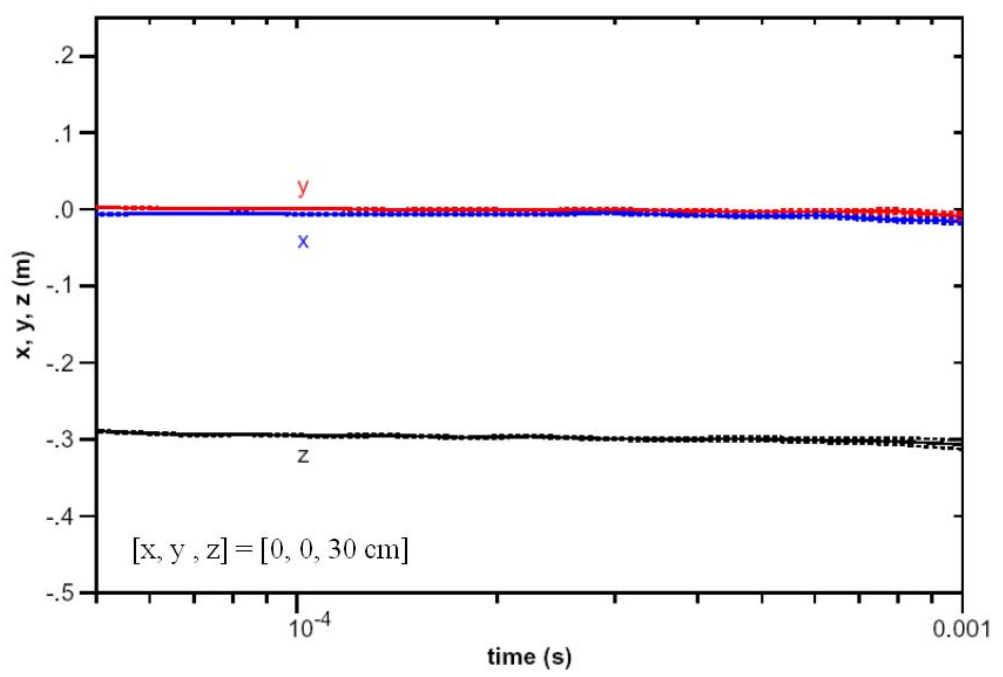

Figure 4.3.4a: Inversion results for the position of 9 inch $(23 \mathrm{~cm})$ steel ellipsoid.

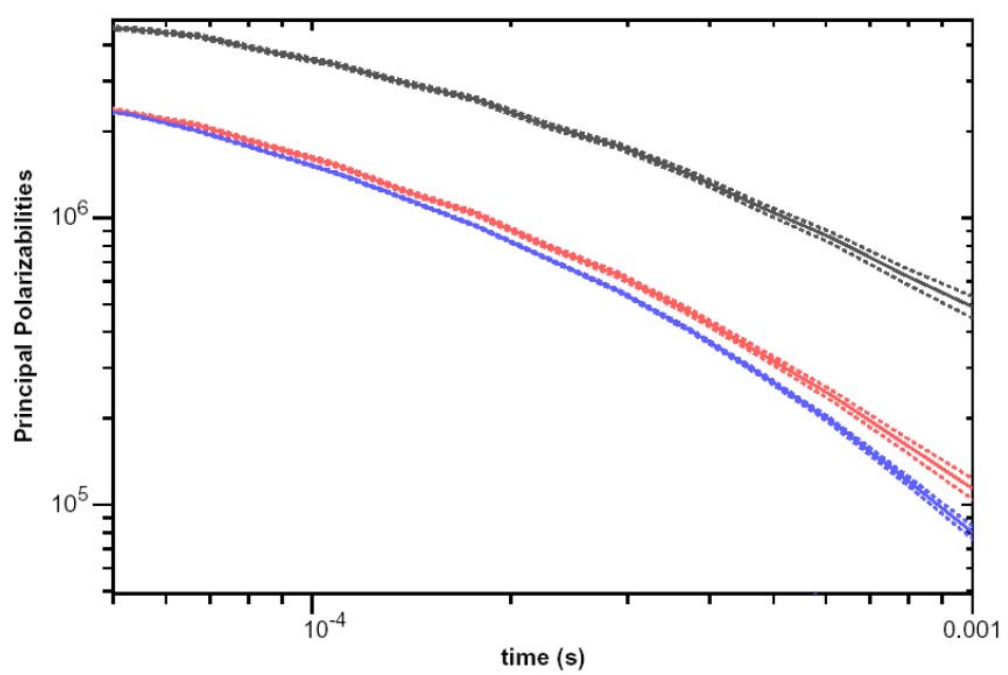

Figure 4.3.4b: Inversion results for the principal polarizabilities of 9 inch $(23 \mathrm{~cm})$ steel ellipsoid. 


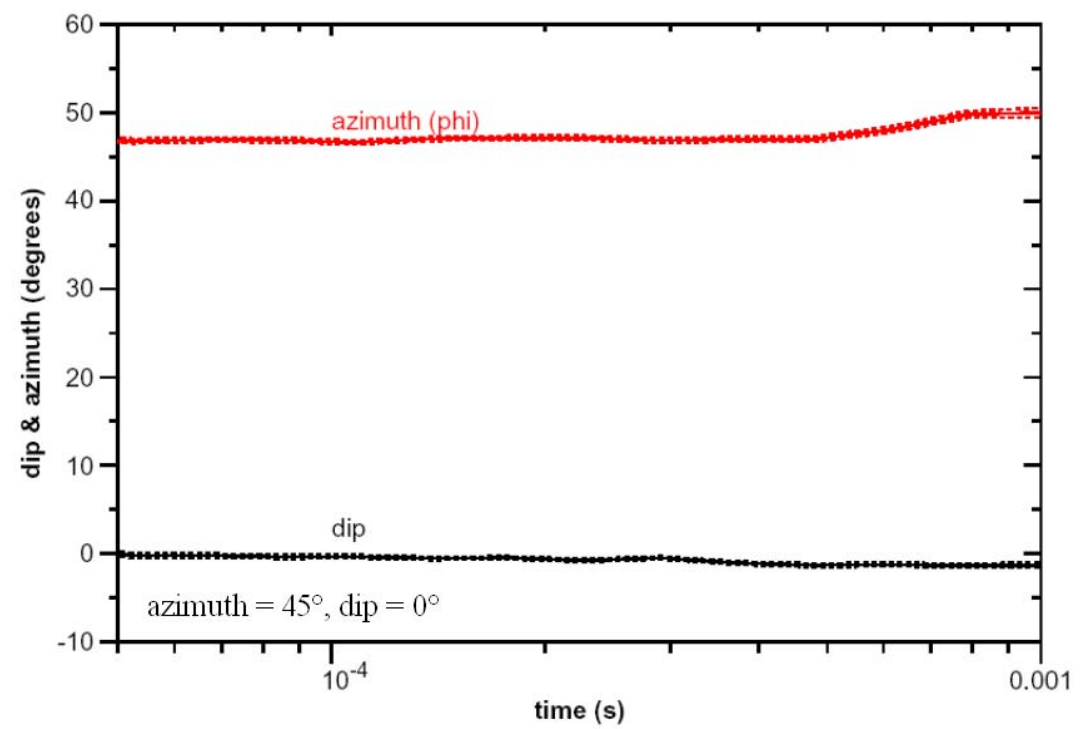

Figure 4.3.4c: Inversion results for the orientation of 9 inch $(23 \mathrm{~cm})$ steel ellipsoid.

Although the system is by no means optimized and the noise levels are well above those anticipated in a final system, we then placed the ellipsoid at a depth of $45 \mathrm{~cm}$ and again horizontal and at an azimuth of $45^{\circ}$. This time the center of the ellipsoid was displaced $25 \mathrm{~cm}$ in $\mathrm{x}$ and $\mathrm{y}$ from the centerline, or $\mathrm{z}$-axis. These positions were not measured with high accuracy so the location, Figure 4.3.5a, at early time can be assumed to be more accurate than the intended value. The recovered polarizabilities, Figure 4.3.5b, clearly show the correct apparent aspect ratio but there is a difference in the two minor polarizabilities that is larger than their respective errors. We do not understand this result at present. Early time estimates of the dip (error less than $\pm 1^{\circ}$ ) and azimuth (error less than $\pm 5^{\circ}$ ), Figure $4.3 .5 \mathrm{c}$, are excellent. All the results become very noisy beyond about $2 \times 10^{-4}$ seconds as the transient signals from such a relatively deep target drop into the noise.

These results, especially the last for an off-center oriented ellipsoid at the depth of $45 \mathrm{~cm}$, clearly show that the multi element system can detect the object and more importantly, determine its principal polarizabilities and their directions. Moreover, it is evident that these quantities can be obtained over a time window that will allow subsequent inversion for the true physical aspect ratio, the size and the ratio of $\sigma / \mu$. 


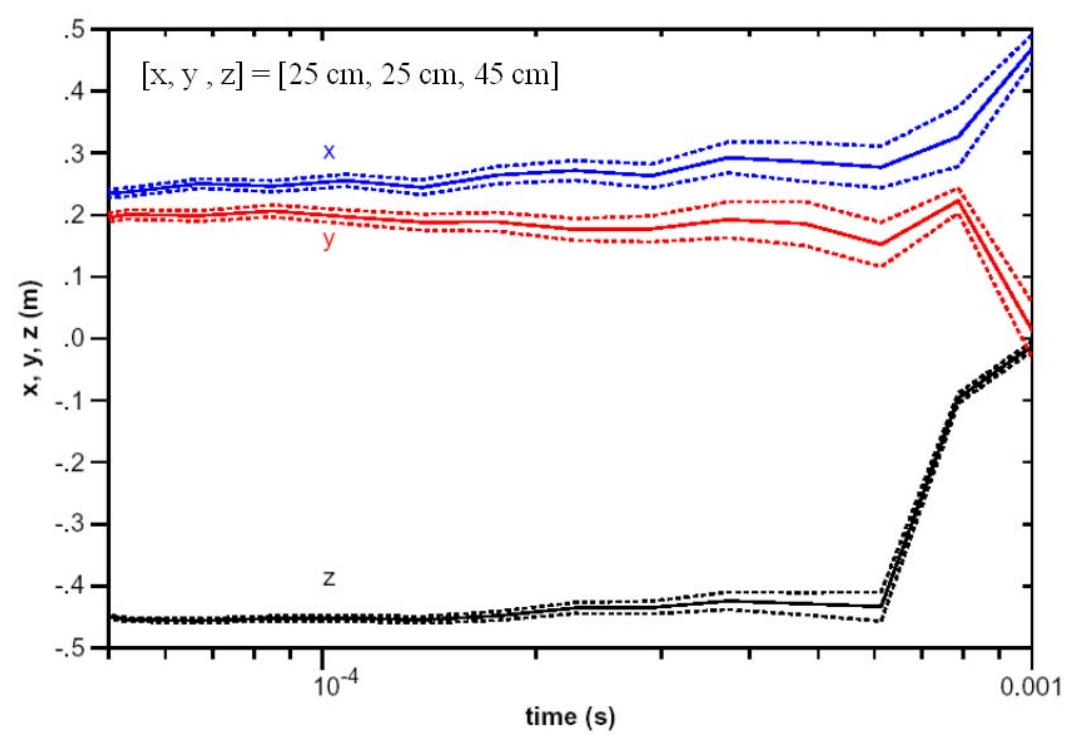

Figure 4.3.5a: Inversion results for the position of 9 inch $(23 \mathrm{~cm})$ steel ellipsoid with offset.

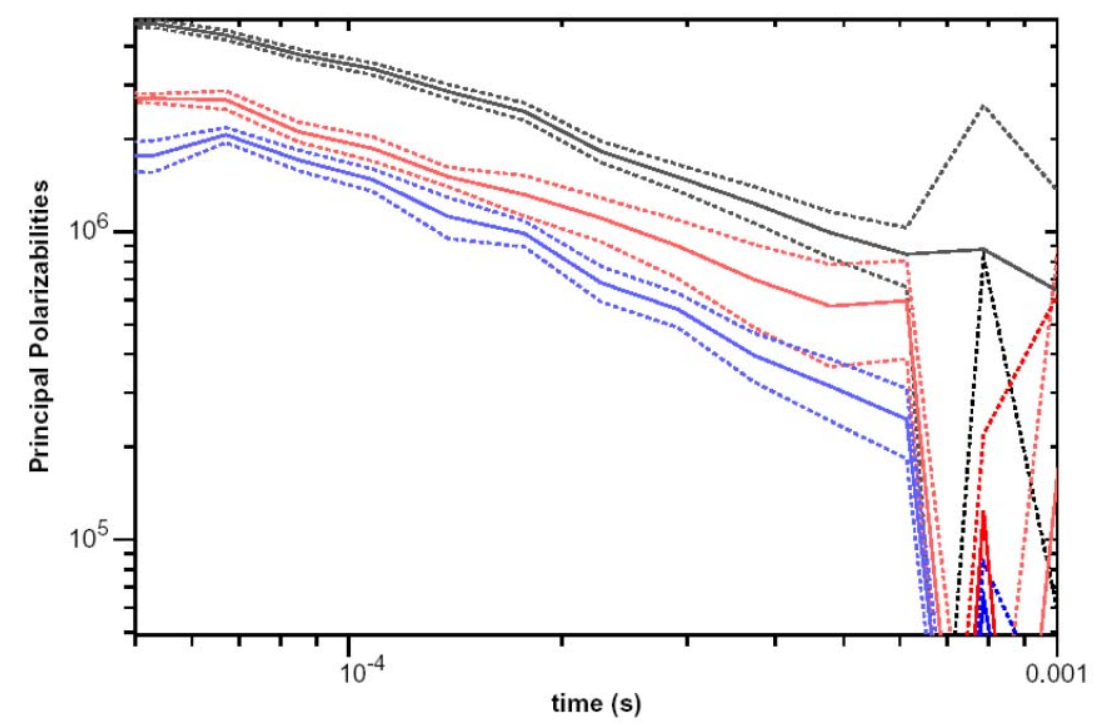

Figure 4.3.5b: Inversion results for the principal polarizabilities of 9 inch $(23 \mathrm{~cm})$ steel ellipsoid with offset. 


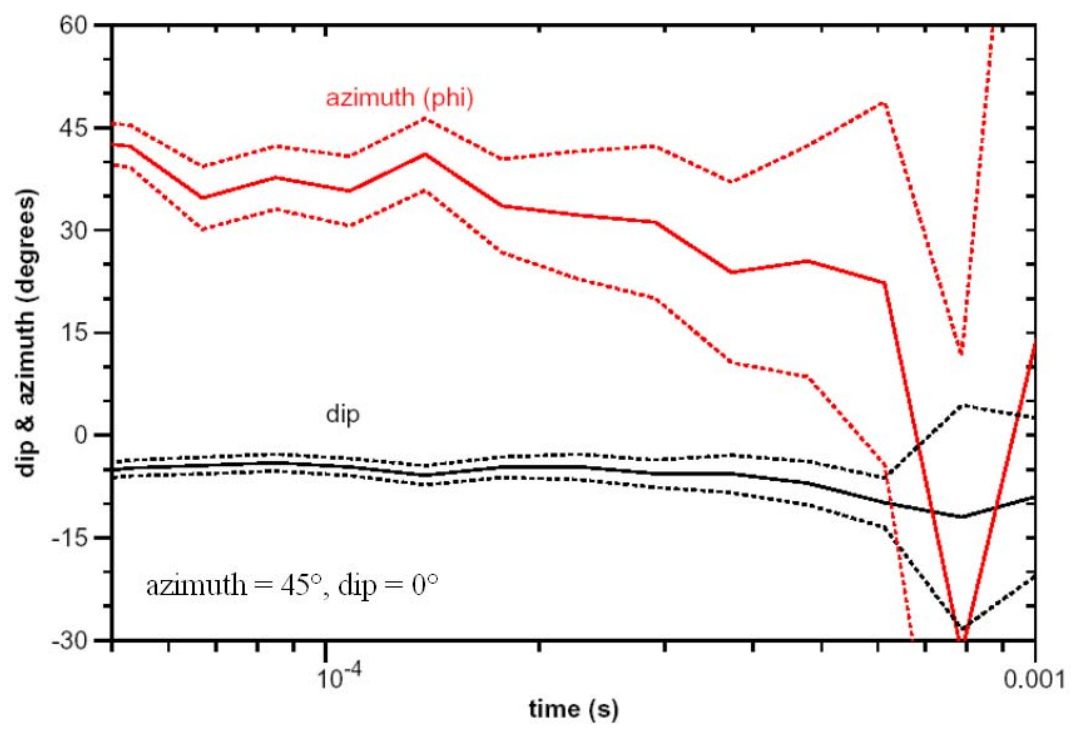

Figure 4.3.5c: Inversion results for the orientation of 9 inch $(23 \mathrm{~cm})$ steel ellipsoid with offset. 


\section{CONCLUSIONS AND ACCOMPLISHMENTS}

The most significant finding are detailed in Section 2 of this report where we use the principal dipole moments model to evaluate the improvements in system performance that can be obtained with use of multiple receivers and/or transmitters. The other most significant result here is that the depth of detection can be doubled compared to a conventional coaxial transmitter - receiver system if one uses a 3 component transmitter as well as a 3 component receiver.

An optimal system is one that allows the most accurate location and classification of the target at the lowest cost in terms of the system size, weight, and power. Basically we are taking the "most bang for your buck" approach. Although we are beginning to see traditional optimization possibilities in coil size and system bandwidth, i.e. a particular setting is better than all others, a more limited approach may have to be taken to the overall system design. This implies increasing system complexity, weight, and power to the point of diminishing returns because an absolutely optimum value for some of the parameters may not exist. Thus increasing the number of sensors and/or transmitters beyond a certain level may not detract from system performance but does not improve it either.

A powerful simulator has been implemented for determining the optimum transmitter-receiver configuration for UXO detection and characterization. Another simulator has been developed for analyzing the role of bandwidth and noise in determining the spectral response of the principal dipole moments.

The major conclusions from research and development are:

1) A multicomponent transmitter-receiver system is essential for the identification of the principal dipole moments of a target, be it UXO or clutter. A complex target can be detected, located, and defined.

2) A three component loop transmitter with 5 small vector sensors suffices to uniquely determine the principal dipole moments of a target. Stand-alone equipment recovers all target parameters from a single position.

3) Similar detection/characterization results can be obtained, with different configurations, using deployment on a 2D grid, on a profile line, or in a stand alone mode.

4) The utility of grid or line deployment is limited by positioning accuracy. 
5) For maximum utility a system capable of stand alone detection/characterization seems best because:

i) it can be used effectively when access does not permit precise line or grid operation.

ii) its sensitivity is independent of absolute position.

iii) when used in line or grid mode its sensitivity is superior to any other configuration.

6) ) $\mathrm{AdB} / \mathrm{dt}$ sensor is preferable.

7) The bandwidth of the receiver and its associated electronics have a profound effect on the secondary field transient and, in-turn, on the accurate recovery of the distinctive properties of the transient that enable the identification of the spectral properties of the target.

8) A bandwidth of at least four decades appears to be necessary to describe adequately the spectral response of the principal moments. Low frequencies are required to identify the permeability of the target and this in-turn requires a low pulse repetition rate for the transmitted fields.

9) The large bandwidth admits more noise and thus requires higher transmitter power to maintain an adequate signal to noise ratio.

10) Target size, ratio $\sigma / \mu_{\mathrm{r}}$, and true physical aspect ratio can be determined.

11) The ground response imposes an early time limit on the time window for target discrimination.

\section{ACKNOWLEDGMENTS}

This work was supported by the Office of Management, Budget and Evaluation, of the U.S. Department of Energy under Contract No. DE-AC03-76SF00098, and U.S. Department of Defense under Strategic Environmental Research and Development Program Project No. UX-1225 and Contract Nos. W74RDV093447299 and W74RDV30452524. 


\section{REFERENCES}

Barrow, B., Khadr, N., and Nelson, H.H., 1996, Performance of electromagnetic induction sensors for detecting and characterizing UXO: Proceedings of UXO Forum 1996, 308-314, Williamsburg, VA.

Baum, C. E., 1999, Low frequency near-field magnetic scattering from highly conducting, but not perfectly conducting bodies, in Baum, C. E., Ed., Detection and Identification of Visually Obscured Targets, Taylor and Francis, Philadelphia, 163-217.

Becker, A., Gasperikova, E., Morrison, H.F., Smith, J.T., 2002, Configuring the AEM System: Partners in Environmental Technology Technical Symposium and Workshop, Washington D.C.

Bell, T. H., Barrow, B., and Khadr, N., 1998, Shape-based classification and discrimination of subsurface objects using electromagnetic induction: IGARSS, Seattle, WA, July 6-10.

Gasperikova, E., 2003, A new-generation EM system for the detection and classification of buried metallic objects: SEG Expanded Abstracts, pp. 2379-2382.

Gasperikova, E., Becker, A., Morrison, H.F., Smith, J.T., 2003, EM sensors for detection and characterization of buried metallic objects: Partners in Environmental Technology Technical Symposium and Workshop, Washington D.C., p. 36.

Gasperikova, E., Becker, A., Morrison, H.F., Smith, J.T., 2005, A multisensor system for the detection and characterization of UXO: SAGEEP, Atlanta.

Glerc, G. and Gilbert,D, 1964. La Contre-reaction de Flux Appliquee aux Bobines a Noyau Magnetique: Annales de Geophysique, 20, 499-502. 
Harrington, R. F., 1968, Field computation by moment methods: The MacMillan Co., New York.

Jackson, J. D., Classical Electrodynamics, Second Edition, John Wiley and Sons, New York, 1975.

Keiswetter, D., Won, I.J., Barrow, B., and Bell, T.H., 1999, Object identification using multifrequency emi data: Proceedings of SAGEEP ‘99, 743-751, Oakland, March 14-18.

Khadr, N., Barrow, B. J., Bell, T. H., and Nelson, H. H., 1998, Target shape classification using electromagnetic induction sensor data: Proceedings of UXO Forum 1998.

Landau, L. D., and Lifshitz, E. M., Electrodynamics of Continuous Media, AddisonWesley, Reading, 1960.

McNeill, J.D., and Bosnar, M., 1996, Application of time domain electromagnetic techniques to UXO detection: Proceedings of UXO Forum 1996, Williamsburg, VA.

Morrison, H.F., Becker, A., Smith, J.T., Gasperikova, E., 2002. Detection and classification of buried metallic objects: EAGE Expanded Abstracts, Florence.

Morrison, H.F., Becker, A., Gasperikova, E., Smith, J.T., 2004, A multisensor system for the detection and characterization of UXO: Partners in Environmental Technology Technical Symposium and Workshop, Washington D.C.

Newman, G. A. and Alumbaugh, D. L., 1997, Three-dimensional massively parallel electromagnetic inversion - I. Theory, Geophys. J. Int., 128, 345-354.

Pasion, L. R., and Oldenburg, D. W., 2001, Locating and determining dimensionality of UXOs using time domain electromagnetic fields: Journal of Environmental and Engineering Geophysics, 6, 91-102. 
Press, W. H., Flannery, B. P., Teukolsky, S. A., and Vetterling, W. T., Numerical Recipes, the Art of Scientific Computing, Cambridge University Press, Cambridge, 1986.

Smith, T.J., Allan, W., and Schultz, A., 1994, Inversion of waveform data using an empirical distribution evolutionary algorithm, Eos, 75, no. 44, p. 457.

Smith, J.T., and Morrison, H.F., 2004, Estimating equivalent dipole polarizabilities for the inductive response of isolated conductive bodies: IEEE Trans. Geosci. Remote Sensing, Vol. 42, p. 1208-1214.

Smith, J.T., and Morrison, H.F., Becker, A., 2004a, Parametric forms and the inductive response of a permeable conducting sphere: Journal of Env. And Engin. Geophysics, 9, p. 213-216.

Smith, J.T., and Morrison, H.F., Becker, A., 2004b, Resolution depths for some transmitter-receiver configurations: IEEE Trans. Geosci. Remote Sensing, Vol. 42, p. $1215-1221$.

Smith, J.T., and Morrison, H.F., Becker, A., 2005, Optimizing receiver configurations for resolution of equivalent dipole polarizabilities in situ: IEEE Trans. Geosci. Remote Sensing, submitted for publication.

Smith, J.T., and Morrison, H.F., 2005a, Approximating spheroid inductive responses using spheres: Geophysics, submitted for publication.

Smith, J.T., and Morrison, H.F., 2005b, Worse case objects for characterization using magnetic induction: IEEE Trans. Geosci. Remote Sensing, to be submitted.

Snyder, D.D., MacInnes, S., Urquhart, S., and Zonge, K.L., 1999, Possibilities for UXO classification using characteristic modes of the broad-band electromagnetic induction 
response: A New Technology Applications Conference on the Science and Technology of Unexploded Ordnance (UXO) Removal and Site Remediation, Outrigger Wailea Resort, Maui.

Ware, G.H., 2000, EM-63 data processing for UXO discrimination: Interim report, ESTCP, October 5 .

Watson, G. S., Statisitics on Spheres, Wiley-Interscience, 1983.

Won, I.J., Keiswetter, D.A., Hanson, D.R., Novikova, E., and Hall, T.M., 1997, GEM-3: a monostatic broadband electromagnetic induction sensor: Journal of Environmental and Engineering Geophysics, 2, 53-64. 


\section{LIST OF FIGURES}

Page

Figure 1.3.1: Schematic diagram of the simulator.

Figure 1.3.2: Schematic diagram of the optimization process. ………………………….....

Figure 1.3.3: Schematic diagram of the verification process. …………………………......

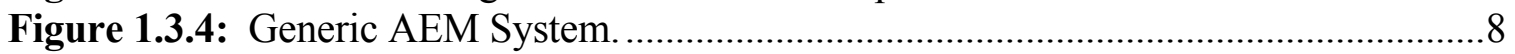

Figure 1.3.5: Schematics of the bench test acquisition setup............................................10

Figure 1.3.6: Photo of the prototype system. ……………………………………….....10

Figure 2.1.1: Squared data misfit $\chi^{2}$ as a function of candidate object center position $\mathrm{r}_{0}$, for $1 \mathrm{~m}^{2}$ square transmitter loop, concentric vertical dipole receiver system sited on a $9 \mathrm{x}$

9 grid, centered $1 \mathrm{~m}$ above a $12 \mathrm{~cm}$ steel sphere (detail).................................................

Figure 2.1.2: Squared data misfit $\chi^{2}$ as a function of candidate object center position $\mathrm{r}_{0}$, for $1 \mathrm{~m}^{2}$ square transmitter loop, 2 coaxial vertical dipole receiver system sited on a 9 x 9 grid, centered $1 \mathrm{~m}$ above a $12 \mathrm{~cm}$ steel sphere (detail).

Figure 2.2.1: Relative rms polarizability uncertainty $\xi$ as a function of sphere center depth below transmitter and receiver for a $6 \mathrm{~cm}$ radius steel sphere. Also, relative uncertainty in vertical moment $\mathrm{dm}_{\mathrm{zz}} / \mathrm{dt}$ (dashed), and in horizontal moments $\mathrm{dm}_{\mathrm{xx}} / \mathrm{dt}$ and $\mathrm{dm}_{\mathrm{yy}} / \mathrm{dt}$ (dotted).

Figure 2.2.2: Depths to $5 \%, 10 \%$, and $20 \%$ rms uncertainty in polarizability as a function of sphere radius for steel spheres below a 9 x 9 grid of transmitter-receiver system placements, for a $1 \mathrm{~m}^{2}$ square loop transmitter with a concentric vertical dipole receiver.

Figure 2.2.3: Depths to $5 \%, 10 \%$, and $20 \%$ uncertainty in sphere center depth, as a function of sphere center depth below center of transmitter-receiver placement grid, for same system and grid as in Figure 2.2.2.

Figure 2.2.4: Depths to $10 \%$ rms polarizability uncertainty and to $10 \%$ uncertainty in center depth, for thin horizontal non-magnetic disk with same vertical polarizability $\mathrm{dm}_{\mathrm{zz}} / \mathrm{dt}$ at $610 \mu \mathrm{s}$, as spheres of Table I, plotted as a function of the corresponding sphere's radius.

Figure 2.2.5: Depths to $10 \%$ rms polarizability uncertainty and to $10 \%$ uncertainty in center depth, as a function of radius for steel spheres below a $1 \mathrm{~m}^{2}$ square transmitter loop with two coaxial vertical component receivers $0.2 \mathrm{~m}$ apart vertically, on same grid as in Figure 2.2.2.

Figure 2.2.6: Depths to $10 \%$ polarizability uncertainty and to $10 \%$ uncertainty in center depth, as a function of sphere radius, for $1 \mathrm{~m}^{2}$ loop transmitter system with two vertical component receivers $0.566 \mathrm{~m}$ apart on diagonal in plane of transmitter loop, on same grid as in Figure 2.2.2.

Figure 2.2.7: Depths to $10 \%$ polarizability uncertainty and to $10 \%$ uncertainty in depth as a function of sphere radius, for $1 \mathrm{~m}^{2}$ loop transmitter system with 3 component concentric receiver, on same grid as in Figure 2.2.2. .

Figure 2.2.8: Depths to $10 \%$ polarizability uncertainty and to $10 \%$ uncertainty in depth, as a function of sphere radius, for three orthogonal $1 \mathrm{~m}^{2}$ loop transmitter system with 
vertical component receiver at horizontal loop center, on same grid as in Figure 2.2.2.

Figure 2.2.9: Depths to $10 \%$ polarizability uncertainty and to $10 \%$ uncertainty in depth, as a function of sphere radius, for 3 transmitter loop system with 3 component receiver at

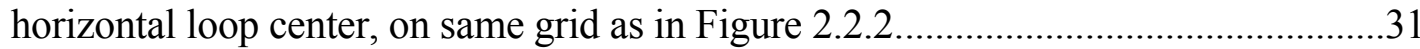

Figure 3.1.1: Induced magnetization and currents in 3D bodies.......................................33

Figure 3.2.1: Depth to $10 \%$ polarizability uncertainty and $10 \%$ uncertainty in depth as a function of sphere radius for TxTyTz-Bz and TxTyTz - BxByBz systems.................37

Figure 3.2.2

Figure 3.2.3: Principal polarizations (PDM) and location for a dipping ellipsoid on $9 \times 9$ grid using a single horizontal loop receiver within a horizontal loop transmitter........38

Figure 3.2.4: Rms uncertainty in polarizability as a function of position on $9 \times 9$ grid using simple in-loop configuration.

Figure 3.2.5: Rms uncertainty in polarizability as a function of position on $9 \times 9$ grid using 3 orthogonal transmitters and 3 orthogonal receivers.

Figure 3.2.6: Rms uncertainty in polarizability as a function of position using two transmitters and three receivers in a profile mode.

Figure 3.2.7: Rms uncertainty in polarizability as a function of position using three transmitters and four receivers in a stand alone mode.

Figure 3.2.8: Rms uncertainty in polarizability as a function of orientation of an elongate target at a depth of $1 \mathrm{~m}$.

Figure 3.2.9: Rms uncertainty in polarizability as a function of position using three transmitters and five receivers in a stand alone mode.

Figure 3.2.10: Rms uncertainty in polarizability as a function of orientation of a target at $1.6 \mathrm{~m}$ depth using three transmitters and five receivers in a stand alone mode

Figure 3.2.11: Rms uncertainty in polarizability as a function of position using three transmitters and five receivers in a regular pattern dictated by transmitter frame.

Figure 3.2.12: Rms uncertainty in polarizability as a function of position using four independent horizontal loops and 9 vertical receivers

Figure 3.2.13: Relative rms polarizability uncertainty as a function of instrument location

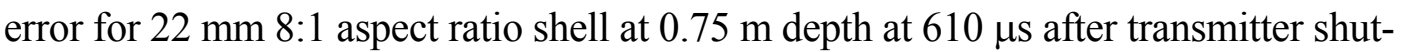
off.

Figure 3.2.14: Relative rms depth uncertainty as a function of instrument location error for $22 \mathrm{~mm} \mathrm{8:1} \mathrm{aspect} \mathrm{ratio} \mathrm{shell} \mathrm{at} 0.75 \mathrm{~m}$ depth at $610 \mu$ s after transmitter shut-off. ......47

Figure 3.3.1: Normalized secondary fields (real and imaginary components) as a function of frequency for $37 \mathrm{~mm}$ aluminum spherical shell of various thicknesses at $0.75 \mathrm{~m}$ depth.

Figure 3.3.2: Normalized magnetic field response as a function of time for $37 \mathrm{~mm}$ aluminum spherical shell of various thicknesses at $0.75 \mathrm{~m}$ depth.

Figure 3.3.3: Normalized $\mathrm{dB} / \mathrm{dt}$ response as a function of time for $37 \mathrm{~mm}$ aluminum spherical shell of various thicknesses at $0.75 \mathrm{~m}$ depth.

Figure 3.3.4: Normalized magnetic field response as a function of frequency for $37 \mathrm{~mm}$ magnetic spherical shell of various thicknesses at $0.75 \mathrm{~m}$ depth.

Figure 3.3.5: Normalized magnetic field response as a function of time for $37 \mathrm{~mm}$ magnetic spherical shell of various thicknesses at $0.75 \mathrm{~m}$ depth. 
Figure 3.3.6: Normalized $\mathrm{dB} / \mathrm{dt}$ response as a function of time for $37 \mathrm{~mm}$ magnetic spherical shell of various thicknesses at $0.75 \mathrm{~m}$ depth...........................................55

Figure 3.3.7: Amplitude of $\mathrm{dB} / \mathrm{dt}$ response for $37 \mathrm{~mm}$ sphere, horizontal and vertical shells $3: 1$ aspect ratio at the depth of $0.75 \mathrm{~m}$ as a function of time together with responses for $10 \Omega$-m and $100 \Omega$-m half-space. .56

Figure 3.3.8: Amplitude of magnetic field response for $37 \mathrm{~mm}$ sphere, horizontal and vertical shells $3: 1$ aspect ratio at the depth of $0.75 \mathrm{~m}$ as a function of time together with responses for $10 \Omega$-m and $100 \Omega$-m half-space.

Figure 3.3.9: Amplitude of $\mathrm{dB} / \mathrm{dt}$ response for $22 \mathrm{~mm}$ sphere, horizontal and vertical shells $8: 1$ aspect ratio at the depth of $0.75 \mathrm{~m}$ as a function of time together with responses for $10 \Omega$-m and $100 \Omega$-m half-space.

Figure 3.3.10: Amplitude of magnetic field response for $22 \mathrm{~mm}$ sphere, horizontal and vertical shells $8: 1$ aspect ratio at the depth of $0.75 \mathrm{~m}$ as a function of time together with responses for $10 \Omega$-m and $100 \Omega$-m half-space...

Figure 3.3.11: Amplitude of $\mathrm{dB} / \mathrm{dt}$ response for $105 \mathrm{~mm}$ sphere, horizontal and vertical shells $4: 1$ aspect ratio at the depth of $2.65 \mathrm{~m}$ as a function of time together with responses for $10 \Omega-\mathrm{m}$ and $100 \Omega$-m half-space.

Figure 3.3.12: Amplitude of magnetic field response for $105 \mathrm{~mm}$ sphere, horizontal and vertical shells $4: 1$ aspect ratio at the depth of $2.65 \mathrm{~m}$ as a function of time together with responses for $10 \Omega$-m and $100 \Omega$-m half-space.

Figure 3.3.13: Amplitude of magnetic field response for $155 \mathrm{~mm}$ sphere, horizontal and vertical shells $4.4: 1$ aspect ratio at the depth of $4.55 \mathrm{~m}$ as a function of time together with responses for $10 \Omega$-m and $100 \Omega$-m half-space.

Figure 3.3.14: Amplitude of magnetic field response for $155 \mathrm{~mm}$ sphere, horizontal and vertical shells $4.4: 1$ aspect ratio at the depth of $4.55 \mathrm{~m}$ as a function of time together with responses for $10 \Omega$-m and $100 \Omega$-m half-space.

Figure 3.4.1: Equivalent sphere response.

Figure 3.5.1: Transient response of a repetitive square waveform..................................65

Figure 3.5.2: The amplitudes for four representative spheres at four different depths plotted as a function of repetition period.

Figure 3.5.3: Repetitive waveform.

Figure 3.5.4: Uncertainties in (a) $\log (\mathrm{r})$, (b) $\log (\sigma / \mu)$, and (c) $\log (\sigma \mu)$ for $160 \mathrm{~mm}$ sphere, resonant frequency $\mathrm{f}_{0}=3.2 \mathrm{kHz}$, and critically damped receiver.

Figure 3.5.5: Uncertainties in (a) $\log (\mathrm{r}),(\mathrm{b}) \log (\sigma / \mu)$, and (c) $\log (\sigma \mu)$ for $160 \mathrm{~mm}$ sphere, resonant frequency $\mathrm{f}_{0}=33 \mathrm{kHz}$, and critically damped receiver.

Figure 3.6.1: Squared weighted data misfit as a function of trial sphere conductivity and relative permeability for bipolar data with an $80 \mathrm{~Hz}$ repetition rate, a 0.4 duty cycle square pulse and a $32 \mathrm{k}$ radian $/ \mathrm{s}$ resonant frequency critically damped receiver using data from $0.47 \mathrm{~ms}$ to $3.75 \mathrm{~ms}$, for synthetic data from a $160 \mathrm{~mm}$ diameter steel sphere.

Figure 3.7.1: Schematic of the bandwidths for (a) B sensor, and (b) critically damped $\mathrm{dB} / \mathrm{dt}$ sensor.

Figure 3.7.2: A typical TEM system: It consists of a transmitter with a "step-off" current waveform, an induction sensor defined by its electrical parameters L, r, C, and a "loop" target defined by its time constant $\tau$. 
Figure 3.7.3: Bandpass characteristics for a critically damped detector from Figure 3.7.2

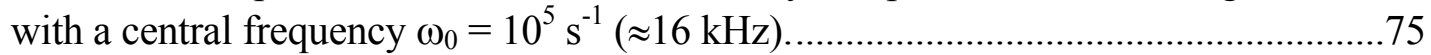

Figure 3.7.4: Sensor response to a step transition in primary flux for the induction detector from Figure 3.7.2.

Figure 3.7.5: Distortion of the secondary field transient for a $100 \mu$ s target by a critically damped detector tuned to about $16 \mathrm{kHz}$ on linear and on log-linear scales. .................78

Figure 3.7.6: Earliest time of detection, to $1 \%$ or $10 \%$ accuracy for $100 \mu$ s target.............79

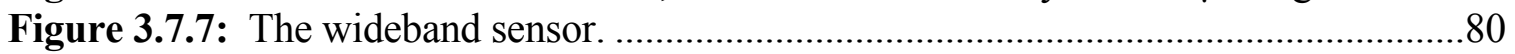

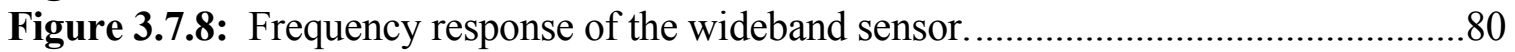

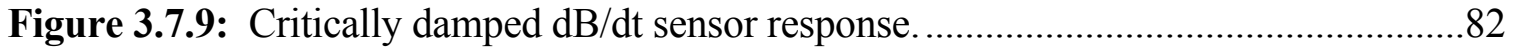

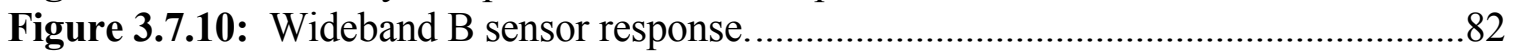

Figure 4.2.1: Schematics of the bench test acquisition setup. ..........................................85

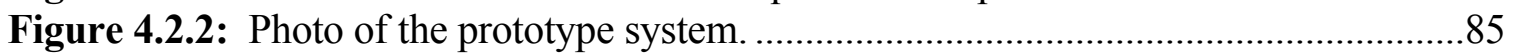

Figure 4.2.3: Half sine waveform of prototype system - repetition rate of $90 \mathrm{~Hz}$, relative pulse length is 0.18 , and maximum current $A=19$ Amperes......................................86

Figure 4.3.1: The 9 inch $(23 \mathrm{~cm})$ long and 3 inches $(7.6 \mathrm{~cm})$ in diameter steel ellipsoid. 87

Figure 4.3.2a: Inversion results for the position of $18 \mathrm{~cm}$ diameter shorted multi-turn loop.

Figure 4.3.2b: Inversion results for the principal moments of $18 \mathrm{~cm}$ diameter shorted multi-turn loop.

Figure 4.3.2c: Inversion results for the moment orientations of $18 \mathrm{~cm}$ diameter shorted multi-turn loop.

Figure 4.3.3a: Inversion results for the position of $138 \mathrm{~mm}$ diameter copper sphere........90

Figure 4.3.3b: Inversion results for the principal moments of $138 \mathrm{~mm}$ diameter copper sphere.

Figure 4.3.4a: Inversion results for the position of 9 inch $(23 \mathrm{~cm})$ steel ellipsoid. ...........91

Figure 4.3.4b: Inversion results for the principal polarizabilities of 9 inch $(23 \mathrm{~cm})$ steel

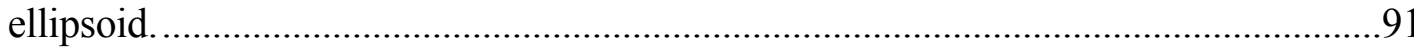

Figure 4.3.4c: Inversion results for the orientation of 9 inch $(23 \mathrm{~cm})$ steel ellipsoid........92

Figure 4.3.5a: Inversion results for the position of 9 inch $(23 \mathrm{~cm})$ steel ellipsoid with offset.....

Figure 4.3.5b: Inversion results for the principal polarizabilities of 9 inch $(23 \mathrm{~cm})$ steel ellipsoid with offset.

Figure 4.3.5c: Inversion results for the orientation of 9 inch $(23 \mathrm{~cm})$ steel ellipsoid with offset.

Figure A.1.1.1: Largest 26 eigenvalues of $\hat{\mathbf{K}}$ scaled to inverse decay times $\lambda$ as a function of aspect ratio, for prolate spheroids of conductivity $\sigma=3.510^{7} \Omega^{-1} \mathrm{~m}^{-1}$ and with a 6 $\mathrm{cm}$ equatorial radius, with equation (A.1.10) truncated at polynomial order $\mathrm{p}=7 \ldots .108$

Figure A.1.1.2: Receiver coil voltage as a function of time for a simulated Geonics EM-61 system with coils $0.6 \mathrm{~m}$ above a $6 \mathrm{~cm}$ radius aluminum sphere. Previous results (large circles). Modified code results summed over 196 modes (dashed). Analytic (solid).

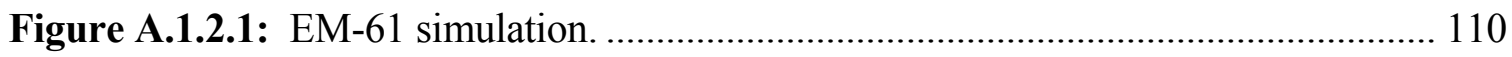

Figure A.1.4.1: Transient responses for B and $\mathrm{dB} / \mathrm{dt}$ for $6 \mathrm{~cm}$ conductive permeable steel

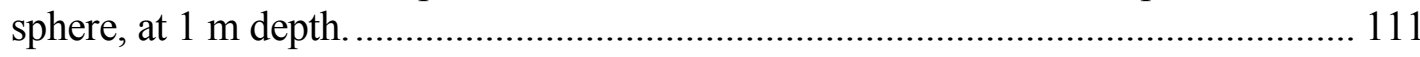


Figure A.1.4.2: Transient responses for B and $\mathrm{dB} / \mathrm{dt}$ for $6 \mathrm{~cm}$ conductive permeable steel sphere, for varying shell thickness, at $1 \mathrm{~m}$ depth...................................................... 112 Figure A.1.4.3: Graphical user interface for calculating responses of a spherical target with an arbitrary transmitter-receiver configuration.............................................. 113 


\section{APPENDIX 1: NUMERICAL MODELS}

\section{A.1.1 Improvements to the Weichman-Blackhawk MFT Code}

P.B. Weichman of Blackhawk Geophysics made available source code for an integral equation method for computing the secondary (scattered) fields due to electromagnetic induction in conductive and possibly magnetic spheroids. The code expands Cartesian components of the electric field (and of the magnetic field when necessary) in terms of a set of polynomials $\mathrm{f}_{\mathrm{i}}(\mathbf{r})$;

$$
\mathbf{E}(\mathbf{r})=\sum_{i=1}^{\infty} \sum_{j=1}^{3} \eta_{i j} f_{i}(\mathbf{r}) \hat{\mathbf{x}}_{\mathrm{j}}
$$

where $\hat{\mathbf{x}}_{\mathrm{j}}$ is a unit vector in the $\mathrm{j}^{\prime}$ th Cartesian coordinate direction. In its course of computations, the code finds eigenvalues and eigenfunctions of an equation of form

$$
\mathbf{E}(\mathbf{r})=\left(\sigma^{\prime}-\sigma_{\mathrm{b}}^{\prime}\right)\left[\frac{4 \pi \mu_{\mathrm{b}} \lambda}{\mathrm{c}^{2}} \mathbf{I}-\frac{\nabla \nabla \cdot}{\sigma_{\mathrm{b}}^{\prime}}\right] \int_{\text {spheroid }} \mathrm{g}_{\mathrm{b}}\left(\mathbf{r}, \mathbf{r}^{\prime}\right) \mathbf{E}(\mathbf{r}) \mathrm{d} \mathbf{r}^{\prime}
$$

(in Gaussian units), where

$$
\sigma^{\prime}=\sigma-\frac{\lambda \varepsilon}{4 \pi}
$$

and $\mathrm{g}_{\mathrm{b}}\left(\mathbf{r}, \mathbf{r}^{\prime}\right)$ is the Green's function for a scalar Helmholz equation in the background medium $\left(\sigma_{\mathrm{b}}, \varepsilon_{\mathrm{b}}, \mu_{\mathrm{b}}\right)$. Terms involving $\left(\mu-\mu_{\mathrm{b}}\right) \mathbf{H}\left(\mathbf{r}^{\prime}\right)$, arising for magnetic spheroids, have been omitted for simplicity of exposition. Integrals of form

$$
\mathrm{I}_{\mathrm{i}}\left(\mathbf{r}_{\mathrm{k}}\right) \equiv \int_{\text {spheroid }} \mathrm{g}_{\mathrm{b}}\left(\mathbf{r}_{\mathrm{k}}, \mathbf{r}^{\prime}\right) \mathrm{f}_{\mathrm{i}}\left(\mathbf{r}^{\prime}\right) \mathrm{d} \mathbf{r}^{\prime}
$$

are evaluated over a set of $m$ points $\mathbf{r}_{\mathrm{k}},(\mathrm{k}=1, \mathrm{~m})$, with the Green's function approximated within the spheroid as $1 /\left(4 \pi \mid \mathbf{r}-\mathbf{r}^{\prime}\right)$;

$$
\mathrm{I}_{\mathrm{i}}(\mathbf{r}) \approx \mathrm{I}_{\mathrm{i}}^{\prime}(\mathbf{r}) \equiv \frac{1}{4 \pi} \int_{\text {spheroid }} \frac{\mathrm{f}_{\mathrm{i}}\left(\mathbf{r}^{\prime}\right)}{\left|\mathbf{r}^{\prime}-\mathbf{r}\right|} \mathrm{d}^{\prime}
$$

For $f_{i}\left(\mathbf{r}^{\prime}\right)$ a polynomial of order $p$ in $x, y$, and $z\left(\right.$ e.g., $\left.x^{\alpha} y^{\beta} z^{p-\alpha-\beta}\right), \mathbf{I}_{i}^{\prime}(\mathbf{r})$ is a polynomial of order $\mathrm{p}+2$ or lower within the spheroid, so can be expanded as

$$
\mathrm{I}_{\mathrm{i}}^{\prime}(\mathbf{r})=\sum_{\mathrm{i}^{\prime}=1}^{\mathrm{n}_{\mathrm{p}+2}} \gamma_{\mathrm{i}^{\prime}}^{(\mathrm{i})} \mathrm{f}_{\mathrm{i}^{\prime}}(\mathbf{r})
$$


where $\mathrm{n}_{\mathrm{p}+2}$ is the number of linearly independent polynomials in $\mathrm{x}, \mathrm{y}$, and $\mathrm{z}$, of order $\mathrm{p}+2$ or less. Coefficients $\gamma_{\mathrm{i}^{i}}{ }^{\left({ }^{\mathrm{i}}\right)}$ are found by numerically integrating (A.1.5) at the points $\mathbf{r}_{\mathrm{k}},(\mathrm{k}=$ $1, \mathrm{~m}$ ), for $\mathrm{m} \geq \mathrm{n}_{\mathrm{p}+2}$, and inverting (A.1.6) for $\gamma_{\mathrm{i}^{\prime}}{ }^{(\mathrm{i})}, \mathrm{i}^{\prime}=1, \mathrm{n}_{\mathrm{p}+2}$. With $\gamma_{\mathrm{i}}{ }^{\left({ }^{\mathrm{i})}\right.}$ computed, it is straightforward to evaluate

$$
\nabla \nabla \cdot \hat{\mathbf{x}}_{\mathrm{j}} \mathrm{I}_{\mathrm{i}}^{\prime}(\mathbf{r})=\sum_{\mathrm{i}^{\prime}=1}^{\mathrm{n}_{\mathrm{p}+2}} \gamma_{\mathrm{i}^{\prime}}^{(\mathrm{i})} \nabla \nabla \cdot \hat{\mathbf{x}}_{\mathrm{j}} \mathrm{f}_{\mathrm{i}^{\prime}}(\mathbf{r})
$$

This is re-expanded in the form

$$
\nabla \nabla \cdot \hat{\mathbf{x}}_{\mathrm{j}} \mathrm{I}_{\mathrm{i}}^{\prime}(\mathbf{r})=\sum_{\mathrm{i}^{\prime}=1}^{\mathrm{n}_{\mathrm{p}}} \sum_{\mathrm{k}=1}^{3} \gamma_{\mathrm{i}^{\prime} \mathrm{jk}}^{\prime(\mathrm{i})} \mathrm{f}_{\mathrm{i}^{\prime}}(\mathbf{r}) \hat{\mathbf{x}}_{\mathrm{k}},
$$

by expressing the needed derivatives of each function $\mathrm{f}_{\mathrm{i}}(\mathbf{r})$ in terms of the basis functions $\mathrm{f}_{\mathrm{i}}(\mathbf{r})$. Using expansions (A.1.1), (A.1.6), and (A.1.8) in equation (A.1.2) yields (A.1.9)

$$
\sum_{\mathrm{i}=1}^{\infty} \sum_{\mathrm{j}=3}^{3} \eta_{\mathrm{ij}} \mathrm{f}_{\mathrm{i}}(\mathbf{r}) \hat{\mathbf{x}}_{\mathrm{j}}=\left(\sigma^{\prime}-\sigma_{\mathrm{b}}^{\prime}\right) \sum_{\mathrm{i}=1}^{\infty} \sum_{\mathrm{j}=1}^{3}\left[\frac{4 \pi \mu_{\mathrm{b}} \lambda}{\mathrm{c}^{2}} \sum_{\mathrm{i}^{\prime}=1}^{\infty} \gamma_{\mathrm{i}^{\prime}}^{(\mathrm{i})} \mathrm{f}_{\mathrm{i}^{\prime}}(\mathbf{r}) \hat{\mathbf{x}}_{\mathrm{j}}-\frac{1}{\sigma_{\mathrm{b}}^{\prime}} \sum_{\mathrm{i}^{\prime}=1}^{\infty} \sum_{\mathrm{k}=1}^{3} \gamma_{\mathrm{i}^{\prime} j \mathrm{j}}^{\prime(\mathrm{i})} \mathrm{f}_{\mathrm{i}^{\prime}}(\mathbf{r}) \hat{\mathbf{x}}_{\mathrm{k}}\right] \eta_{\mathrm{ij}} .
$$

Truncating the expansion to the $\mathrm{n}_{\mathrm{p}}$ polynomials of order $\mathrm{p}$ or less yields (A.1.10)

$$
\sum_{\mathrm{i}=1}^{\mathrm{n}_{\mathrm{p}}} \sum_{\mathrm{j}=3}^{3} \eta_{\mathrm{ij}} \mathrm{f}_{\mathrm{i}}(\mathbf{r}) \hat{\mathbf{x}}_{\mathrm{j}}=\left(\sigma^{\prime}-\sigma_{\mathrm{b}}^{\prime}\right) \sum_{\mathrm{i}=1}^{\mathrm{n}_{\mathrm{p}}} \sum_{\mathrm{j}=1}^{3}\left[\frac{4 \pi \mu_{\mathrm{b}} \lambda}{\mathrm{c}^{2}} \sum_{\mathrm{i}^{\prime}=1}^{\mathrm{n}_{\mathrm{p}}} \gamma_{\mathrm{i}^{\prime}}^{(\mathrm{i})} \mathrm{f}_{\mathrm{i}^{\prime}}(\mathbf{r}) \hat{\mathbf{x}}_{\mathrm{j}}-\frac{1}{\sigma_{\mathrm{b}}^{\prime}} \sum_{\mathrm{i}^{\prime}=1}^{\mathrm{n}_{\mathrm{p}}} \sum_{\mathrm{k}=1}^{3} \gamma_{\mathrm{i}^{\prime} j \mathrm{k}}^{(\mathrm{i})} \mathrm{f}_{\mathrm{i}^{\prime}}(\mathbf{r}) \hat{\mathbf{x}}_{\mathrm{k}}\right] \eta_{\mathrm{ij}} .
$$

As originally programmed, integral (A.1.6) was fitted by least squares to the $n_{p}$ polynomials of order $\mathrm{p}$ or less, at points $\mathbf{r}_{\mathrm{k}}, \mathrm{k}=1, \mathrm{~m}$, yielding approximate values for the first $n_{p}$ coefficients $\gamma_{i^{i}}{ }^{(i)}$. As a consequence, the final summation over $i^{\prime}$ in equation (A.1.10), was missing terms of orders $\mathrm{p}-1$ and $\mathrm{p}$ arising from the $\nabla \nabla$. term in equation (A.1.2).

Equation (A.1.10) is satisfied throughout the spheroid when satisfied separately for the coefficients of each pair $\mathrm{f}_{\mathrm{i}}(\mathbf{r}) \hat{\mathbf{x}}_{\mathrm{j}}, \mathrm{i}=1, \mathrm{n}_{\mathrm{p}}, \mathrm{j}=1,3$, yielding a matrix equation in a vector e of coefficients $\eta_{\mathrm{ij}}$;

$$
\mathbf{e}=\hat{\mathbf{K}} \mathbf{e}
$$

(sic). When the set of polynomials, $\mathrm{f}_{\mathrm{i}}(\mathbf{r})$, is orthogonal over the spheroid (and normalized), the resulting matrix $\hat{\mathbf{K}}$ is symmetric, so has real eigenvalues and eigenvectors. 
As originally programmed, the expansion was in terms of monomials $f_{i}(\mathbf{r})=x^{\alpha_{i}} y^{\beta_{i}} z^{\gamma_{i}}$ with $\alpha_{i}+\beta_{i}+\gamma_{i} \leq p$. In that basis, truncation of equation (A.1.9) in equation (A.1.10) and formation of equation (A.1.11), results in a matrix $\hat{\mathbf{K}}$ which is not symmetric, and has complex eigenvalues. Keeping terms up to $\mathrm{i}=\mathrm{n}_{\mathrm{p}+2}$ in expansion (A.1.6), and the corresponding terms in the first summation over $\mathrm{i}^{\prime}$ on the right hand side of equation (A.1.10) by summing to $\mathrm{i}^{\prime}=\mathrm{n}_{\mathrm{p}+2}$, expressing (A.1.10) in a basis of polynomials orthogonal over the spheroid, truncating in that basis to polynomials of order less than or equal to $\mathrm{n}_{\mathrm{p}}$, and re-expressing in the monomial basis, is sufficient to ensure that the eigenvalues of $\hat{\mathbf{K}}$ are real within round-off error. It is, however, simpler to leave the problem expressed in terms of the orthogonal basis, as then subroutines taking advantage of the symmetry of $\hat{\mathbf{K}}$ can be used to find its eigenvalues and eigenvectors. With these changes, the leading eigenvalues are stable up to aspect ratios of 28:1 for non-magnetic prolate spheroids (Figure A.1.1.1).

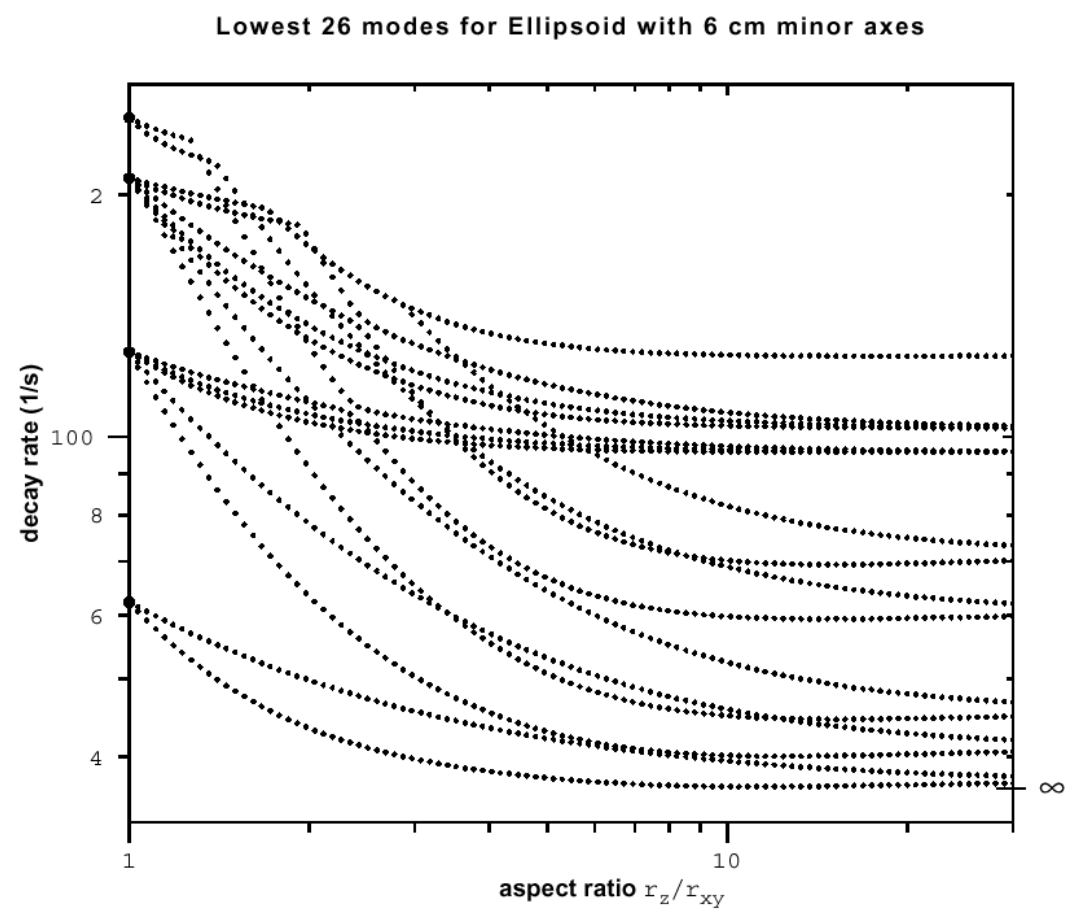

Figure A.1.1.1: Largest 26 eigenvalues of $\hat{\mathbf{K}}$ scaled to inverse decay times $\lambda$ as a function of aspect ratio, for prolate spheroids of conductivity $\sigma=3.510^{7} \Omega^{-1} \mathrm{~m}^{-1}$ and with a 6 $\mathrm{cm}$ equatorial radius, with equation (A.1.10) truncated at polynomial order $\mathrm{p}=7$. 
Once values of inverse decay time $\lambda$ are found for which matrix $\hat{\mathbf{K}}$ has unit eigenvalue(s), the corresponding eigenvectors give the expansion coefficients for the corresponding mode of the spheroid. The excitation of the modes by a transmitter loop of a specified geometry is computed for a ramp-on/ramp-off transmitter current, and the contributions of the different modes to voltages observed in a receiver coil are summed over modes. Figure A.1.1.2 shows results from the modified code (dashed line) using the lowest 196 modes for a $6 \mathrm{~cm}$ radius sphere of conductivity $\sigma=3.510^{7} \Omega^{-1} \mathrm{~m}^{-1}$ (with fundamental time constant $\tau \approx 15 \mathrm{~ms}$ ) at $12 \mathrm{~cm}$ depth below a simulated Geonics EM-61 system. Also shown, are analytic results for the same sphere (solid line), and results from the unmodified code (large circles), both for the same simulated transmitter-receiver system. The modified code results agree to within $10 \% 0.5 \mathrm{~ms}(\tau / 30)$ after transmitter pulse end, and to within $2 \% 3 \mathrm{~ms}(\tau / 5)$ after pulse end. In contrast results for the unmodified code are accurate to within $20 \% 4 \mathrm{~ms}(\approx \tau / 4)$ after pulse end, and worse before that.

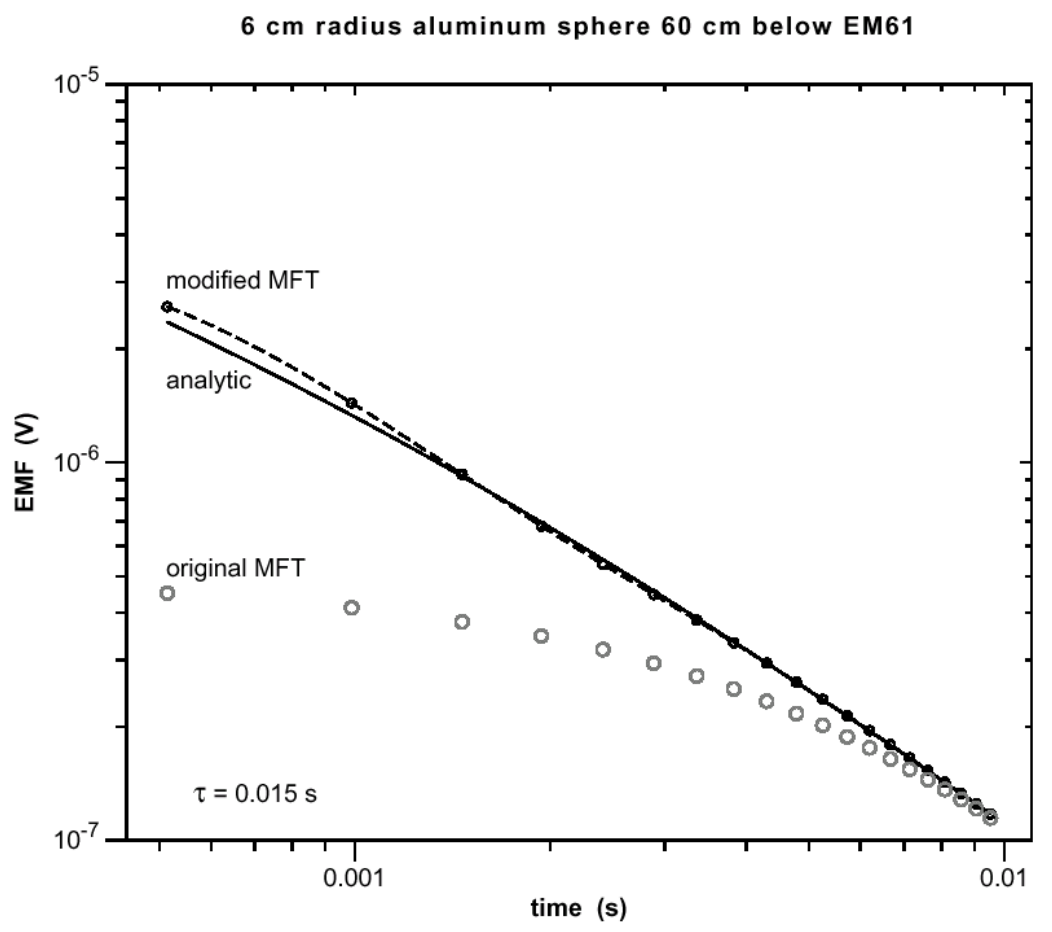

Figure A.1.1.2: Receiver coil voltage as a function of time for a simulated Geonics EM-61 system with coils $0.6 \mathrm{~m}$ above a $6 \mathrm{~cm}$ radius aluminum sphere. Previous results (large circles). Modified code results summed over 196 modes (dashed). Analytic (solid). 
Using the modified code to simulate EM-61 responses on a grid of system locations above a $8 \mathrm{~cm}$ by $24 \mathrm{~cm}$ aluminum prolate spheroid, and inverting for equivalent dipole polarizability, yields an equivalent dipole polarizability that is consistent with the symmetry properties of the spheroid. The resultant equivalent dipole polarizability is centered at the spheroid center, has two equal principal moments and a third, smaller, principal moment with principal direction parallel to the spheroid symmetry axis (see Estimating Equivalent Dipole Polarizabilities Section for details).

\section{A.1.2 Loop-Loop over Dipole Targets}

We have developed a general code for modeling the response of any configuration of finite loops over a target represented by orthogonal dipole moments of any magnitude. An example of the EM-61 response at early time over a simple vertical moment target (e.g. a small horizontal circular sheet) is shown in Figure A.1.2.1. This is the general code for modeling specific T-R configurations such as those described in Section 3.
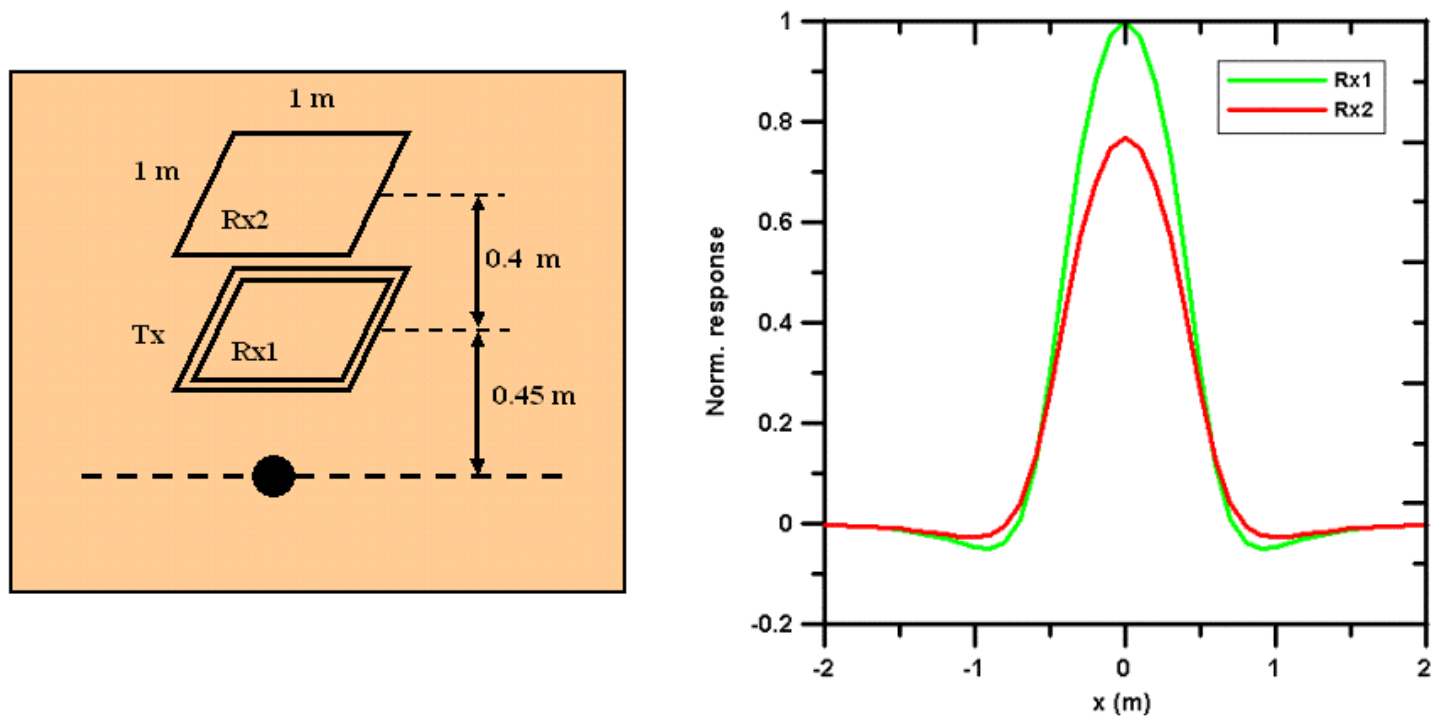

Figure A.1.2.1: EM-61 simulation.

\section{A.1.3 Sheet in layered half space}

We have included a finite loop source in our general code for determining the response of a finite rectangular thin sheet in a layered and conductive half space. This is a 
useful general model for evaluating the ratio of the target response to the half space response and we have used it to show that this ratio decreases as the transmitter receiver separation, or the size of the transmitter loop, increases.

\section{A.1.4 Conductive, permeable spherical shell}

We have completed an analysis of the spectral response of a conductive, permeable, shell in a conductive whole space using the closed form solution we programmed in the Phase 1 part of this project. The source is either a uniform field (approximately valid when the size of the sphere is small compared to the distance to the source) or the field of a dipole (and by superposition, the field of a finite loop).

This code is particularly useful in showing that for typical UXO (usually shells), the response is significantly different at intermediate to late times compared to solids of the same materials. This code has also shown some of the inherent properties of conductive target responses that may influence fundamental system design. For example for a conductive, permeable steel sphere, the transient responses for the field, B, and the time derivative of the field, $\mathrm{dB} / \mathrm{dt}$, are plotted in Figure A.1.4.1 below. Over six decades of time B falls by only 3 orders of magnitude, whereas $\mathrm{dB} / \mathrm{dt}$ falls by over 6 orders of magnitude.
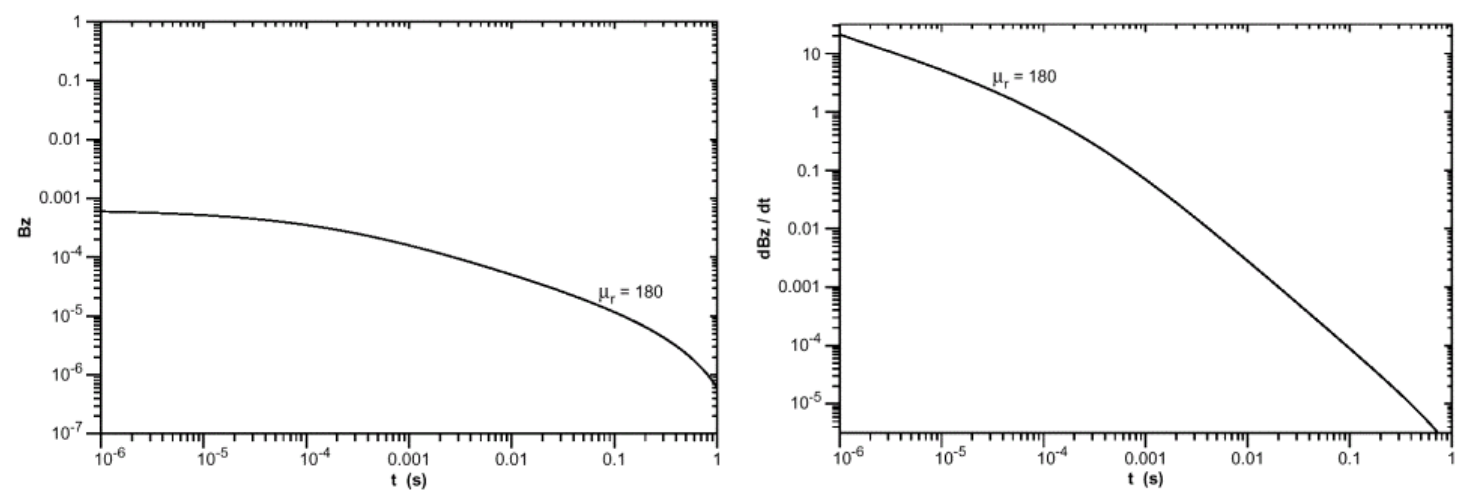

Figure A.1.4.1: Transient responses for $\mathrm{B}$ and $\mathrm{dB} / \mathrm{dt}$ for $6 \mathrm{~cm}$ conductive, permeable steel sphere, at $1 \mathrm{~m}$ depth.

The other important result seen in Figure A.1.4.2, for the same sphere but for varying shell thickness, is that the $\mathrm{dB} / \mathrm{dt}$ response becomes exponential at about $10 \mathrm{msec}$ for a thin shell, whereas it becomes exponential almost a decade earlier in B. From an identification point of view, the properties of the shell are identifiable at earlier times and at 
higher signal levels in $B$ than in $\mathrm{dB} / \mathrm{dt}$. Identifying a target through its spectral response clearly must be done with a shell. To our knowledge, no programs currently exist that can model spheroidal or other shells.
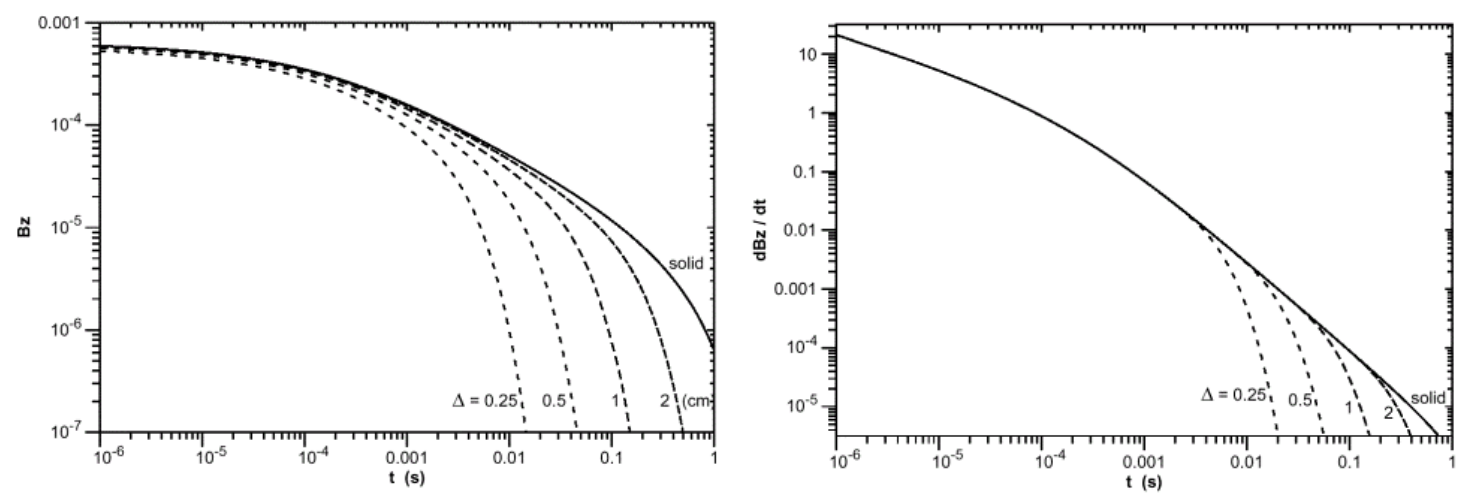

Figure A.1.4.2: Transient responses for $\mathrm{B}$ and $\mathrm{dB} / \mathrm{dt}$ for $6 \mathrm{~cm}$ conductive permeable steel sphere, for varying shell thickness, at $1 \mathrm{~m}$ depth.

We have built a graphical user interface for this modeling code to make it more accessible to the UXO community. A snapshot of the interface is shown in Figure A.1.4.3. (Parties interested in using this software need to send a formal request to LBNL.) The graphical user interface allows the user to model a response of either a solid sphere or a spherical shell at a specified depth. The source can be a dipole, a plane wave, or a loop. The receivers are vertical dipoles that can be placed in any arbitrary position. The response can be calculated either in frequency or time domain. For a stand-alone configuration, the program will calculate a response over a specified range of frequencies or times. In a profile configuration, a response is calculated at one particular frequency or time along the length of the profile. Time domain calculations allow for a "step off," "step on," or "square wave" transmitter current waveform signal. Moreover, the code has the option to calculate a $\mathrm{dB} / \mathrm{dt}$ response as seen through an inductive receiver of a specified center frequency and bandwidth. 


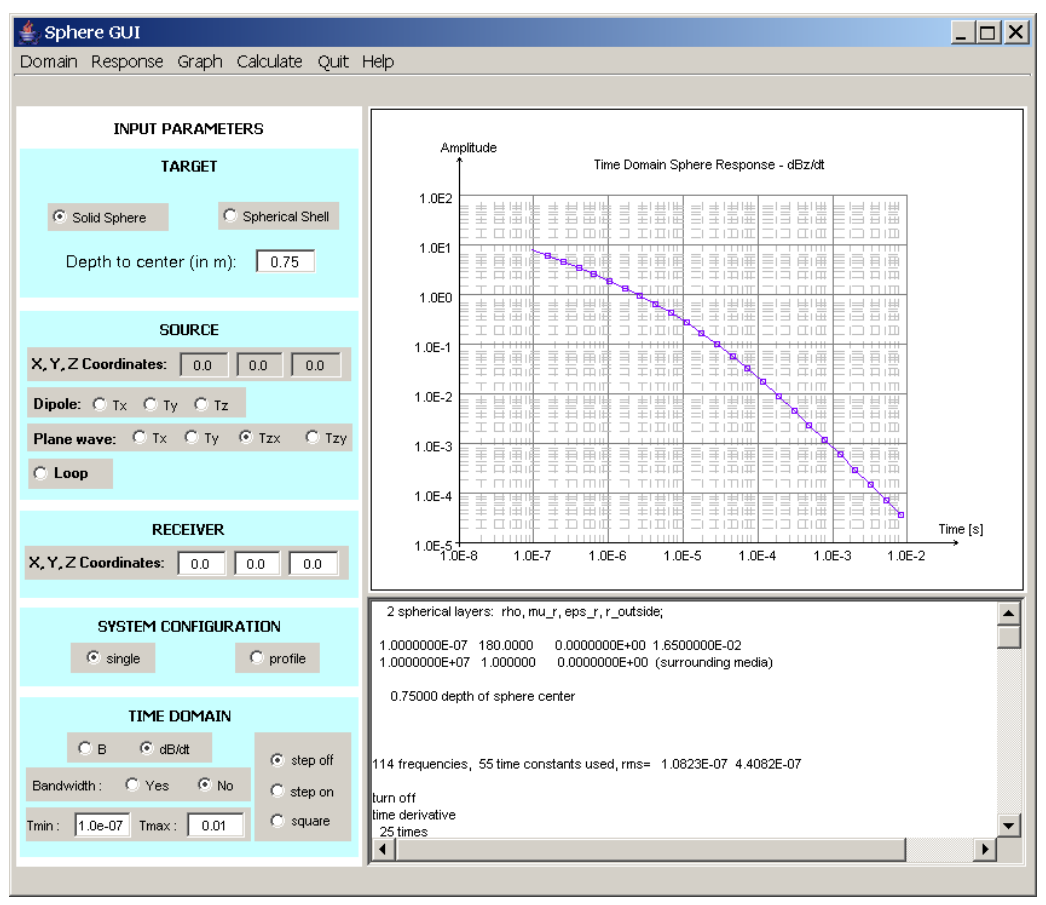

Figure A.1.4.3: Graphical user interface for calculating responses of a spherical target with an arbitrary transmitter-receiver configuration.

\section{A.1.5 3-D half-space of arbitrary conductivity and permeability}

We have obtained the general finite difference 3D code developed by Greg Newman at Sandia National Labs (Newman and Alumbaugh, 1997). We will use this code to model the effects of inhomogeneous ground, particularly inhomogeneities in magnetic susceptibility, on loop-loop systems. This code was recently modified by Newman and promises to greatly accelerate our analysis and understanding of the role of geologic noise in AEM systems for UXO. 